OAK RIDGE NATIONAL LABORATORY

LOCKNIID MATIIN

\section{SNF Shipping Cask Shielding Analysis}

\author{
J. O. Johnson \\ J. V. Pace III
}


This report has been reproduced directly from the best available copy.

Available to DOE and DOE contractors from the Office of Scientific and Technical Information, P.O. Box 62, Oak Ridge, TN 37831; prices available from (615) 576-8401, FTS 626-8401.

Available to the public from the National Technical Information Service, U.S. Department of Commerce, 5285 Port Royal Rd., Springfield, VA 22181.

This report was prepared as an account of work sponsored by an agency of the United States Government. Neither the United States Government nor any agency thereof, nor any of their employees, makes any warranty, express or implied, or assumes any legal liability or responsibility for the accuracy, completeness, or usefulness of any information, apparatus, product, or process disclosed, or represents that its use would not infringe privately owned rights. Reference herein to any specific commercial product, process, or service by trade name, trademark, manufacturer, or otherwise, does not necessarily constltute or imply its endorsement, recommendation, or favoring by the United States Government or any agency thereof. The views and opinions of authors expressed herein do not necessarily state or reflect those of the United States Government or any agency thereof. 
Computational Physics and Engineering Division

\title{
SNF SHIPPING CASK SHIELDING ANALYSIS
}

J. O. Johnson and J. V. Pace III

DATE COMPLETED - November 1995

DATE PUBLISHED - January 1996

Research sponsored by the

Office of Nuclear Energy

U.S. Department of Energy

\author{
Prepared by \\ Oak Ridge National Laboratory \\ Oak Ridge, Tennessee 37831 \\ managed by \\ LOCKHEED MARTIN ENERGY RESEARCH CORP. \\ for the \\ U. S. DEPARTMENT OF ENERGY \\ under contract DE-AC05-960R22464
}




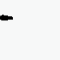


Page LIST OF FIGURES $\ldots \ldots \ldots \ldots \ldots \ldots \ldots \ldots \ldots \ldots \ldots \ldots \ldots, \ldots \ldots \ldots$

LIST OF TABLES $\ldots \ldots \ldots \ldots \ldots \ldots \ldots \ldots \ldots \ldots \ldots \ldots \ldots \ldots \ldots \ldots \ldots \ldots \ldots$

ACKNOWLEDGEMENTS $\ldots \ldots \ldots \ldots \ldots \ldots \ldots \ldots \ldots \ldots \ldots \ldots \ldots \ldots \ldots \ldots$

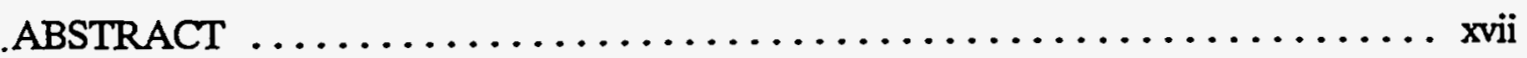

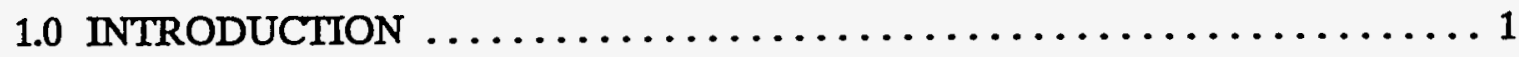

2.0 DESCRIPTION OF THE SHIPPING CASKS $\ldots \ldots \ldots \ldots \ldots \ldots \ldots \ldots \ldots$

3.0 DESCRIPTION OF THE CROSS SECTIONS ................. 5

4.0 DESCRIPTION OF THE RADIATION SOURCES $\ldots \ldots \ldots \ldots \ldots \ldots \ldots 7$

5.0 DESCRIPTION OF CALCULATIONAL MODELS $\ldots \ldots \ldots \ldots \ldots \ldots \ldots$

6.0 DESCRIPTION OF CALCULATIONAL METHOD .............. 13

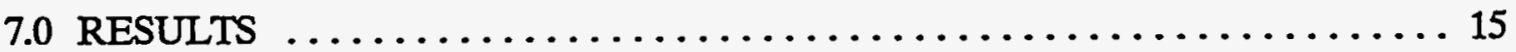

8.0 UNCERTAINTIES . . . . . . . . . . . . . . . . . . . . . 29

9.0 EXAMPLE APPLICATIONS OF DOSE AND THERMAL HEATING DATA

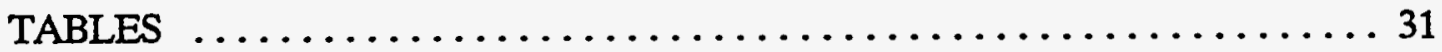

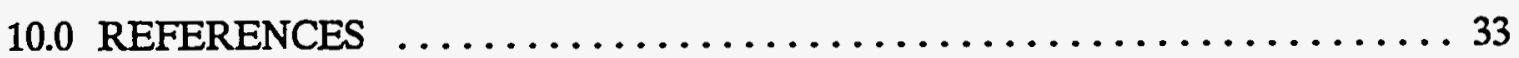

APPENDIX A - Gamma Source Spectra $\ldots \ldots \ldots \ldots \ldots \ldots \ldots \ldots \ldots \ldots$ A 1

APPENDIX B - Major Isotopic Activity (Ci) for the Mixed Fission Product (MFP) Sources from the $50-\mathrm{MWd} / \mathrm{kgU}, 40-\mathrm{kW} / \mathrm{kgU}$ Case. .............. B-1

APPENDIX C - Graphical Representations of the DORT Calculational Models Used in the Shielding Analysis of the SNF Shipping Casks $\ldots \ldots \ldots \ldots \ldots$ C-1

APPENDIX D - Isodose Contours for the Representative Sources Used in the Shielding Analysis of the SNF Shipping Casks $\ldots \ldots \ldots \ldots \ldots \ldots \ldots$ D-1

APPENDIXE - Sample Card Input Data Sets for the Computer Codes Used in the Shielding Analysis of the SNF Shipping Casks $\ldots \ldots \ldots \ldots \ldots \ldots \ldots \ldots$ E-1 


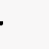




\section{LIST OF FIGURES}

Fiqure

Page

1. Flow Diagram of the SNF Shipping Casks Shielding Analysis $\ldots \ldots \ldots \ldots \ldots$

2. Detector Locations for the Surface and 1-m-Distance Dose Rates Used in the Loop Transport Carrier SNF Shipping Cask Shielding Analysis . . . . . . . . 18

3. Detector Locations for the Surface and 1-m-Distance Dose Rates Used in the In-Pile Loop LITR HB-2 Carrier SNF Shipping Cask Shielding Analysis . . . . 19

4. Detector Locations for the Surface and 1-m-Distance Dose Rates Used in the 6.5-Inch HRLEL Carrier SNF Shipping Cask Shielding Analysis . . . . . . 20

5. Detector Locations for the Surface and 1-m-Distance Dose Rates Used in the HFIR Hot Scrap Carrier SNF Shipping Cask Shielding Analysis . . . . . . . 21

6. Detector Locations for the Surface and 1-m-Distance Dose Rates Used in the 10-Inch ORR Experiment Removal Shield SNF Shipping Cask

Shielding Analysis . . . . . . . . . . . . . . . . . . . . 22

7. Activity Per Initial Gram of Uranium Loading Versus Decay Time for the Five MFP

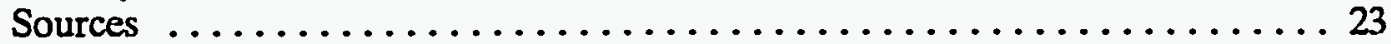

C.1 DORT Calculational Model of the Loop Transport Carrier SNF Shipping

Cask ..................................... C-3

C.2 Expanded View of the DORT Calculational Model of the Loop Transport Carrier Door Plug .............................

C.3 Expanded View of the DORT Calculational Model of the Loop Transport Carrier

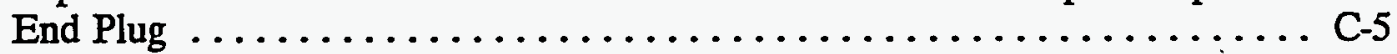

C.4 DORT Calculational Model of the In-Pile Loop LITR HB-2 Carrier SNF Shipping Cask ................................. C-6

C.5 Expanded View of the DORT Calculational Model of the In-Pile Loop LITR HB-2 Carrier Double Doors .......................... C-7

C.6 Expanded View of the DORT Calculational Model of the In-Pile Loop LITR HB-2 Carrier Large Door $\ldots \ldots \ldots \ldots \ldots \ldots \ldots \ldots \ldots \ldots \ldots . \ldots \ldots$

C.7 DORT Calculational Model of the 6.5-Inch HRLEL Carrier SNF Shipping

Cask ............................... C-9 


\section{LIST OF FIGURES (cont.)}

Figure

Page

C.8 Expanded View of the DORT Calculational Model of the 6.5-Inch HRLEL Carrier

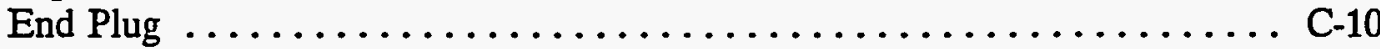

C.9 Expanded View of the DORT Calculational Model of the 6.5-Inch HRLEL Carrier Door ................................ C-11

C.10 DORT Calculational Model of the HFIR Hot Scrap Carrier SNF Shipping Cask

C.11 Expanded View of the DORT Calculational Model of the HFIR Hot Scrap Carrier

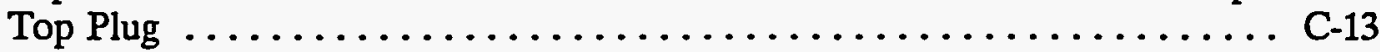

C.12 Expanded View of the DORT Calculational Model of the HFIR Hot Scrap Carrier Bottom Door ............................ C-14

C.13 DORT Calculational Model of the 10-Inch ORR Experiment Removal Shield SNF

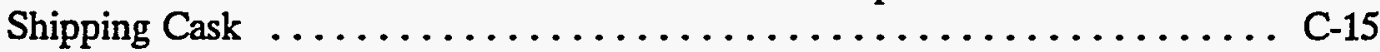

C.14 Expanded View of the DORT Calculational Model of the 10-Inch ORR Experiment

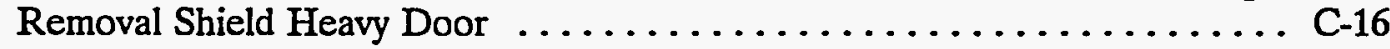

C.15 Expanded View of the DORT Calculational Model of the 10-Inch ORR Experiment Removal Shield Light Door $\ldots \ldots \ldots \ldots \ldots \ldots \ldots \ldots \ldots \ldots \ldots \ldots \ldots \ldots$ C-17

D.1 Isodose Contours for the ${ }^{60} \mathrm{Co}$ Source Packaged in the Loop Transport Carrier SNF Shipping Cask. [Dose units are rem/(h.Ci).] ............... D-3

D.2 Expanded View of the Door Plug Isodose Contours for the ${ }^{60} \mathrm{Co}$ Source Packaged in the Loop Transport Carrier SNF Shipping Cask.

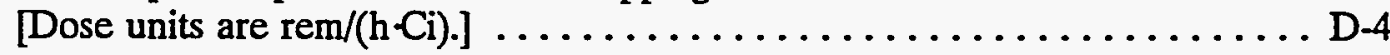

D.3 Expanded View of the End Plug Isodose Contours for the ${ }^{60}$ Co Source Packaged in the Loop Transport Carrier SNF Shipping Cask.

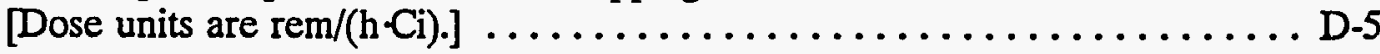

D.4 Isodose Contours for the LWR MFP (25 MWd/kgU) Source Packaged in the Loop Transport Carrier SNF Shipping Cask. [Dose units are rem/(h-Ci).] . . . . . D-6

D.5 Isodose Contours for the LWR MFP (50 MWd/kgU) Source Packaged in the Loop Transport Carrier SNF Shipping Cask. [Dose units are rem/(h-Ci).] . . . . . D-7

D.6 Isodose Contours for the ${ }^{137} \mathrm{Cs}$ Source Packaged in the Loop Transport Carrier SNF Shipping Cask. [Dose units are rem/(h-Ci).] ............... D-8 


\section{LIST OF FIGURES (cont.)}

Figure

Page

D.7 Isodose Contours for the ORR MFP (20\% Enriched ${ }^{235} U$ ) Source Packaged in the Loop Transport Carrier SNF Shipping Cask.

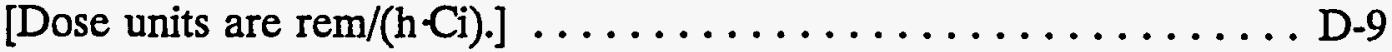

D.8 Isodose Contours for the ORR MFP (40\% Enriched ${ }^{235} \mathrm{U}$ ) Source Packaged in the Loop Transport Carrier SNF Shipping Cask.

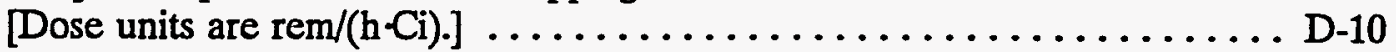

D.9 Isodose Contours for the ORR MFP (93\% Enriched ${ }^{235} \mathrm{U}$ ) Source Packaged in the Loop Transport Carrier SNF Shipping Cask.

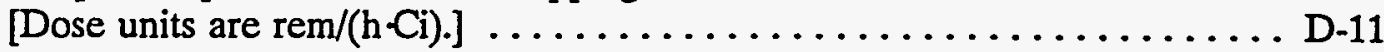

D.10 Isodose Contours for the ${ }^{60} \mathrm{Co}$ Source Packaged in the In-Pile Loop LITR HB-2 Carrier SNF Shipping Cask. [Dose units are rem/(h-Ci).] ........... D-12

D.11 Expanded View of the Double Doors Isodose Contours for the ${ }^{60} \mathrm{Co}$ Source Packaged in the In-Pile Loop LITR HB-2 Carrier SNF Shipping Cask.

[Dose units are rem/(h-Ci).] ....................... D 13

D.12 Expanded View of the Large Door Isodose Contours for the ${ }^{60} \mathrm{Co}$ Source Packaged in the In-Pile Loop LITR HB-2 Carrier SNF Shipping Cask.

$[$ Dose units are rem/(h-Ci) $]$. ...........................

D.13 Isodose Contours for the LWR MFP (25 MWd/kgU) Source Packaged in the In-Pile Loop LITR HB-2 Carrier SNF Shipping Cask.

[Dose units are rem/(h-Ci).] ......................... D 15

D.14 Isodose Contours for the LWR MFP (50 MWd/kgU) Source Packaged in the In-Pile Loop LITR HB-2 Carrier SNF Shipping Cask.

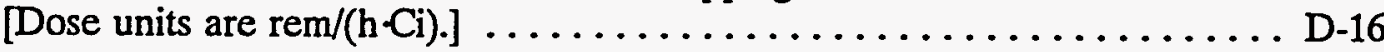

D.15 Isodose Contours for the ${ }^{137} \mathrm{Cs}$ Source Packaged in the In-Pile Loop LITR HB-2 Carrier SNF Shipping Cask. [Dose units are rem/(h.Ci).] . . . . . . . D-17

D.16 Isodose Contours for the ORR MFP (20\% Enriched ${ }^{235} \mathrm{U}$ ) Source Packaged in the In-Pile Loop LITR HB-2 Carrier SNF Shipping Cask.

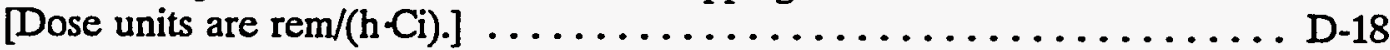

D.17 Isodose Contours for the ORR MFP (40\% Enriched ${ }^{235} \mathrm{U}$ ) Source Packaged in the In-Pile Loop LITR HB-2 Carrier SNF Shipping Cask.

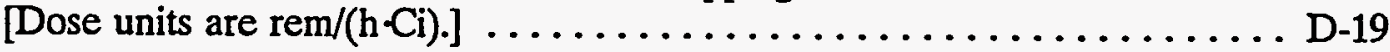

D.18 Isodose Contours for the ORR MFP (93\% Enriched ${ }^{235} U$ ) Source Packaged in the In-Pile Loop LITR HB-2 Carrier SNF Shipping Cask.

[Dose units are rem/(h-Ci).] 


\section{LIST OF FIGURES (cont.)}

Figure

$\underline{\text { Page }}$

D.19 Isodose Contours for the ${ }^{60} \mathrm{Co}$ Source Packaged in the 6.5-Inch HRLEL Carrier SNF Shipping Cask. [Dose units are rem/(h.Ci).] ............. D-21

D.20 Expanded View of the End Plug Isodose Contours for the ${ }^{60} \mathrm{Co}$ Source Packaged in the 6.5-Inch HRLEL Carrier SNF Shipping Cask.

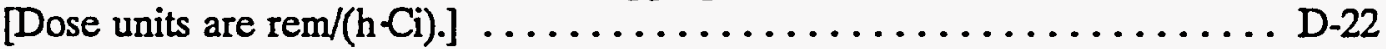

D.21 Expanded View of the Door Isodose Contours for the ${ }^{60} \mathrm{Co}$ Source Packaged in the 6.5-Inch HRLEL Carrier SNF Shipping Cask.

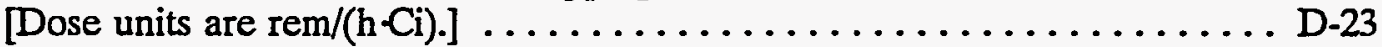

D.22 Isodose Contours for the LWR MFP (25 MWd/kgU) Source Packaged in the 6.5-Inch HRLEL Carrier SNF Shipping Cask. [Dose units are rem/(h-Ci).] . . . . . . D-24

D.23 Isodose Contours for the LWR MFP (50 MWd/kgU) Source Packaged in the 6.5-Inch HRLEL Carrier SNF Shipping Cask. [Dose units are rem/(h-Ci).] . . . . . D D-25

D.24 Isodose Contours for the ${ }^{137} \mathrm{Cs}$ Source Packaged in the 6.5-Inch HRLEL Carrier SNF Shipping Cask. [Dose units are rem/(h-Ci).] . . . . . . . . D-26

D.25 Isodose Contours for the ORR MFP (20\% Enriched ${ }^{235} U$ ) Source Packaged in the 6.5-Inch HRLEL Carrier SNF Shipping Cask.

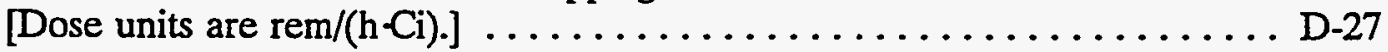

D.26 Isodose Contours for the ORR MFP (40\% Enriched ${ }^{235} U$ ) Source Packaged in the 6.5-Inch HRLEL Carrier SNF Shipping Cask.

[Dose units are rem/(h-Ci).] . . . . . . . . . . . . . . D-28

D.27 Isodose Contours for the ORR MFP (93\% Enriched ${ }^{235} U$ ) Source Packaged in the 6.5-Inch HRLEL Carrier SNF Shipping Cask.

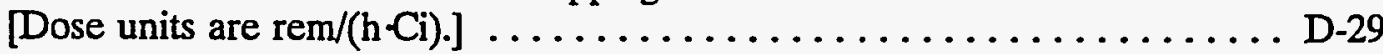

D.28 Isodose Contours for the ${ }^{60} \mathrm{Co}$ Source Packaged in the HFIR Hot Scrap Carrier SNF Shipping Cask. [Dose units are rem/(h-Ci).] ............. D-30

D.29 Expanded View of the Top Plug Isodose Contours for the ${ }^{60} \mathrm{Co}$ Source Packaged in the HFIR Hot Scrap Carrier SNF Shipping Cask.

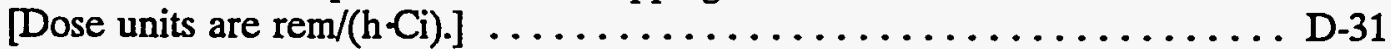

D.30 Expanded View of the Bottom Door Isodose Contours for the ${ }^{60} \mathrm{Co}$ Source Packaged in the HFIR Hot Scrap Carrier SNF Shipping Cask.

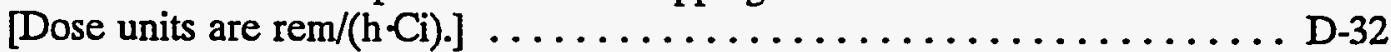

D.31 Isodose Contours for the LWR MFP (25 MWd/kgU) Source Packaged in the HFIR Hot Scrap Carrier SNF Shipping Cask. [Dose units are rem/(h-Ci).] . . . . D-33 


\section{LIST OF FIGURES (cont.)}

Figure

D.32 Isodose Contours for the LWR MFP (50 MWd/kgU) Source Packaged in the HFIR Hot Scrap Carrier SNF Shipping Cask. [Dose units are rem/(h-Ci).] . . . . D D-34

D.33 Isodose Contours for the ${ }^{137} \mathrm{Cs}$ Source Packaged in the HFIR Hot Scrap Carrier SNF Shipping Cask. [Dose units are rem/(h.Ci).] ............. D-35

D.34 Isodose Contours for the ORR MFP (20\% Enriched ${ }^{235} U$ ) Source Packaged in the HFIR Hot Scrap Carrier SNF Shipping Cask.

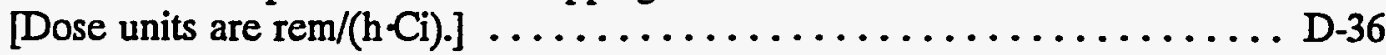

D.35 Isodose Contours for the ORR MFP (40\% Enriched ${ }^{235} \mathrm{U}$ ) Source Packaged in the HFIR Hot Scrap Carrier SNF Shipping Cask.

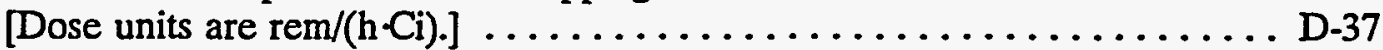

D.36 Isodose Contours for the ORR MFP (93\% Enriched ${ }^{235} U$ ) Source Packaged in the HFIR Hot Scrap Carrier SNF Shipping Cask.

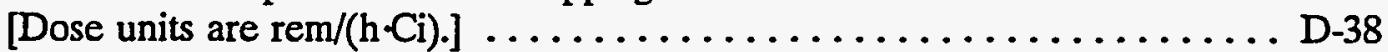

D.37 Isodose Contours for the ${ }^{60} \mathrm{Co}$ Source Packaged in the 10-Inch ORR Experiment Removal Shield SNF Shipping Cask. [Dose units are rem/(h.Ci).] . . . . . . D-39

D.38 Expanded View of the Heavy Shield Section Isodose Contours for the ${ }^{60} \mathrm{Co}$ Source Packaged in the 10-Inch ORR Experiment Removal Shield SNF Shipping Cask.

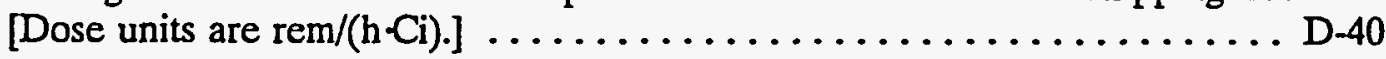

D.39 Expanded View of the Central Light Shield Section Isodose Contours for the ${ }^{60} \mathrm{Co}$ Source Packaged in the 10-Inch ORR Experiment Removal Shield SNF Shipping Cask. [Dose units are $\mathrm{rem} /(\mathrm{h} \cdot \mathrm{Ci}).] \quad \ldots \ldots \ldots \ldots \ldots \ldots \ldots \ldots \ldots$ D-41

D.40 Expanded View of the Light Shield Section Isodose Contours for the ${ }^{60} \mathrm{Co}$ Source Packaged in the 10-Inch ORR Experiment Removal Shield SNF Shipping Cask.

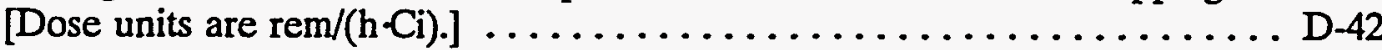

D.41 Expanded View of the Heavy Door Isodose Contours for the ${ }^{60}$ Co Source Packaged in the 10-Inch ORR Experiment Removal Shield SNF Shipping Cask.

[Dose units are $\mathrm{rem} /(\mathrm{h} \cdot \mathrm{Ci}).] \ldots \ldots \ldots \ldots \ldots \ldots \ldots \ldots \ldots \ldots \ldots \ldots \ldots$

D.42 Expanded View of the Light Door Isodose Contours for the ${ }^{60}$ Co Source Packaged in the 10-Inch ORR Experiment Removal Shield SNF Shipping Cask.

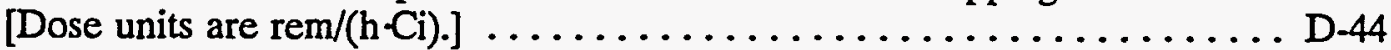

D.43 Isodose Contours for the LWR MFP (25 MWd/kgU) Source Packaged in the 10-Inch ORR Experiment Removal Shield SNF Shipping Cask.

[Dose units are $\mathrm{rem} /(\mathrm{h} \cdot \mathrm{Ci}).] \ldots \ldots \ldots \ldots \ldots \ldots \ldots \ldots \ldots \ldots \ldots \ldots \ldots \ldots$ 


\section{LIST OF FIGURES (cont.)}

Figure

Page

D.44 Isodose Contours for the LWR MFP (50 MWd/kgU) Source Packaged in the 10-Inch ORR Experiment Removal Shield SNF Shipping Cask.

[Dose units are rem/(h-Ci).] ...................... D-46

D.45 Isodose Contours for the ${ }^{137} \mathrm{Cs}$ Source Packaged in the 10-Inch ORR Experiment Removal Shield SNF Shipping Cask. [Dose units are rem/(h.Ci).] . . . . . D-47

D.46 Isodose Contours for the ORR MFP (20\% Enriched ${ }^{235} U$ ) Source Packaged in the 10-Inch ORR Experiment Removal Shield SNF Shipping Cask.

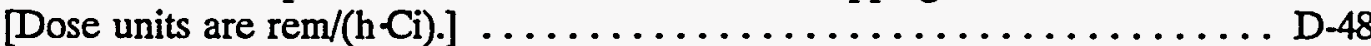

D.47 Isodose Contours for the ORR MFP (40\% Enriched ${ }^{235} U$ ) Source Packaged in the 10-Inch ORR Experiment Removal Shield SNF Shipping Cask.

[Dose units are rem/(h-Ci).] . . . . . . . . . . . . . . . . . . . . D 4 .

D.48 Isodose Contours for the ORR MFP (93\% Enriched ${ }^{235} \mathrm{U}$ ) Source Packaged in the 10-Inch ORR Experiment Removal Shield SNF Shipping Cask.

[Dose units are rem/(h-Ci).] . . . . . . . . . . . . . . . . D-50

E.1 Job Input Stream for the PAL Cross-Section and Activity Manipulation

Program .............................. E-3

E.2 Job Input Stream for the AMP Cross-Section and Activity Manipulation

Program .............................. E-4

E.3 Job Input Stream for the ORIGEN-S Depletion/Burnup Calculation to Define the LWR MFP (25 MWd/kgU) Source Used in the SNF Shipping Casks Shielding Analysis . . . . .......................... E-6

E.4 Job Input Stream for the ORIGEN-S Depletion/Burnup Calculation to Define the LWR MFP (50 MWd/kgU) Source Used in the SNF Shipping Casks Shielding Analysis . . . . . . . . . . . . . . . . . . . . . . . . . . . . E-9

E.5 Job Input Stream for the ORIGEN-S Depletion/Burnup Calculation to Define the ORR MFP (20\% Enriched ${ }^{235} U$ ) Source Used in the SNF Shipping Casks Shielding Analysis ................................. E-12

E.6 Job Input Stream for the ORIGEN-S Depletion/Burnup Calculation to Define the ORR MFP (40\% Enriched ${ }^{235} U$ ) Source Used in the SNF Shipping Casks Shielding Analysis E-15

E.7 Job Input Stream for the ORIGEN-S Depletion/Burnup Calculation to Define the ORR MFP (93\% Enriched ${ }^{235} \mathrm{U}$ ) Source Used in the SNF Shipping Casks Shielding Analysis . . . ........................... E-18 


\section{LIST OF FIGURES (cont.)}

Figure

Page

E.8 Card Input Data for the GIP Cross-Section and Gamma-Ray Heating Activity Mixing Calculation $\ldots \ldots \ldots \ldots \ldots \ldots \ldots \ldots \ldots \ldots \ldots \ldots \ldots \ldots$ E-21

E.9 Card Input Data for the RTFLUM Flux Conversion Utility Program . . . . E E-24

E.10 Card Input Data for the DORT Calculation of the ${ }^{60} \mathrm{Co}$ Source Packaged in the Loop Transport Carrier SNF Shipping Cask ............... E-25

E.11 Card Input Data Changes for the DORT Calculation of the Other Sources Packaged in the Loop Transport Carrier SNF Shipping Cask ............. E-28

E.12 Card Input Data for the FALSTF Calculations of the Surface and One Meter Distance Dose Rates at the Detector Locations Used in the Loop Transport Carrier SNF Shipping Cask Shielding Analysis . . . . . . . . . . . . . E-29

E.13 Card Input Data for the ISOPLOT Isodose Contour Maps for the Loop Transport Carrier SNF Shipping Cask Shielding Analysis . . . . . . . . . . E-30

E.14 Card Input Data for the DORT Calculation of the ${ }^{60} \mathrm{Co}$ Source Packaged in the In-Pile Loop LITR HB-2 Carrier SNF Shipping Cask . . . . . . . . . E-32

E.15 Card Input Data Changes for the DORT Calculation of the Other Sources Packaged in the In-Pile Loop LITR HB-2 Carrier SNF Shipping Cask . . . . . . . . . E-34

E.16 Card Input Data for the FALSTF Calculations of the Surface and One Meter Distance Dose Rates at the Detector Locations Used in the In-Pile Loop LITR HB-2 Carrier SNF Shipping Cask Shielding Analysis .............. E-35

E.17 Card Input Data for the ISOPLOT Isodose Contour Maps for the In-Pile Loop LITR HB-2 Carrier SNF Shipping Cask Shielding Analysis . . . . . . . . . E-36

E.18 Card Input Data for the DORT Calculation of the ${ }^{60} \mathrm{Co}$ Source Packaged in the 6.5 Inch HRLEL Carrier SNF Shipping Cask

E.19 Card Input Data Changes for the DORT Calculation of the Other Sources Packaged in the 6.5-Inch HRLEL Carrier SNF Shipping Cask ............. E-41

E.20 Card Input Data for the FALSTF Calculations of the Surface and One Meter Distance Dose Rates at the Detector Locations Used in the 6.5-Inch HRLEL Carrier SNF Shipping Cask Shielding Analysis . . . . . . . . . . . . E-42

E.21 Card Input Data for the ISOPLOT Isodose Contour Maps for the 6.5-Inch HRLEL Carrier SNF Shipping Cask Shielding Analysis .............. E-43

E.22 Card Input Data for the DORT Calculation of the ${ }^{60} \mathrm{Co}$ Source Packaged in the HFIR Hot Scrap Carrier SNF Shipping Cask 


\section{LIST OF FIGURES (cont.)}

Figure

$\underline{\text { Page }}$

E.23 Card Input Data Changes for the DORT Calculation of the Other Sources Packaged in the HFIR Hot Scrap Carrier SNF Shipping Cask . . . . . . . . . E E-48

E.24 Card Input Data for the FALSTF Calculations of the Surface and One Meter Distance Dose Rates at the Detector Locations Used in the HFIR Hot Scrap Carrier SNF Shipping Cask Shielding Analysis . . . . . . . . . . . . . E-49

E.25 Card Input Data for the ISOPLOT Isodose Contour Maps for the HFIR Hot Scrap Carrier SNF Shipping Cask Shielding Analysis . . . . . . . . . . E E-50

E.26 Card Input Data for the DORT Calculation of the ${ }^{60}$ Co Source Packaged in the 10 Inch ORR Experiment Removal Shield SNF Shipping Cask ......... E-52

E.27 Card Input Data Changes for the DORT Calculation of the Other Sources Packaged in the 10-Inch ORR Experiment Removal Shield SNF Shipping

E.28 Card Input Data for the FALSTF Calculations of the Surface and One Meter Distance Dose Rates at the Detector Locations Used in the 10-Inch ORR Experiment Removal Shield SNF Shipping Cask Shielding Analysis .......... E-55

E.29 Card Input Data for the ISOPLOT Isodose Contour Maps for the 10-Inch ORR Experiment Removal Shield SNF Shipping Cask Shielding Analysis ..... E-56 


\section{LIST OF TABLES}

Table

1. VELM Photon Energy-Group Structure $\ldots \ldots \ldots \ldots \ldots \ldots \ldots \ldots \ldots$

2. Composition of Materials Used in the Radiation Shielding Analysis

of the Spent Nuclear Fuel Shipping Casks $\ldots \ldots \ldots \ldots \ldots \ldots \ldots \ldots \ldots$

3. Radioisotopes Forming Bulk of SNF $\ldots \ldots \ldots \ldots \ldots \ldots \ldots \ldots \ldots$

4. Energy Group and Source Strength for ${ }^{60} \mathrm{Co}$ and ${ }^{137} \mathrm{Cs} \ldots \ldots \ldots \ldots \ldots$

5. Three RERTR Program Miniplate Attributes $\ldots \ldots \ldots \ldots \ldots \ldots \ldots \ldots$

6. Alpha and Beta Heating Rates Per Curie (W/Ci) by Source Type . . . . . . 15

7. Gamma-Ray Heating Rates for the Designated Spent Nuclear Fuel Shipping Cask to be Used to Transfer Radioactive Waste from the SWSA Area Sites, Building 7827 to Building 3525 and for Representative Sources Stored in the SWSA Area Sites,

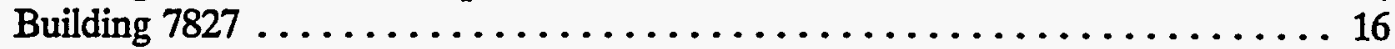

8. Gamma-Ray Dose Rates at Selected Detector Positions $3.81 \mathrm{~cm}$ and $1 \mathrm{~m}$ Away From the Surface of the Loop Transport Carrier Spent Nuclear Fuel Shipping Cask for Representative Sources Stored in the SWSA Area Sites,

Building 7827.

9. Gamma-Ray Dose Rates at Selected Detector Positions $3.81 \mathrm{~cm}$ and $1 \mathrm{~m}$ Away From the Surface of the In-Pile Loop LITR HB-2 Carrier Spent Nuclear Fuel Shipping Cask for Representative Sources Stored in the SWSA Area Sites, Building 7827. 25

10. Gamma-Ray Dose Rates at Selected Detector Positions $3.81 \mathrm{~cm}$ and $1 \mathrm{~m}$ Away From the Surface of the 6.5-Inch HRLEL Carrier Spent Nuclear Fuel Shipping Cask for Representative Sources Stored in the SWSA Area Sites,

Building 7827.

11. Gamma-Ray Dose Rates at Selected Detector Positions $3.81 \mathrm{~cm}$ and $1 \mathrm{~m}$ Away From the Surface of the HFIR Hot Scrap and Spent Nuclear Fuel Shipping Cask for Representative Sources Stored in the SWSA Area Sites, Building 7827. . . . . 27

12. Gamma-Ray Dose Rates at Selected Detector Positions $3.81 \mathrm{~cm}$ and $1 \mathrm{~m}$ Away From the Surface of the 10-Inch ORR Experiment Removal Shield Spent Nuclear Fuel Shipping Cask for Representative Sources Stored in the SWSA Area Sites,

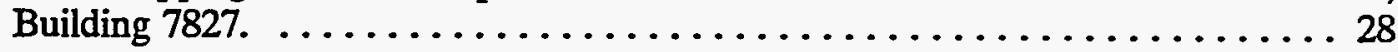

A.1 Gamma Source Spectra (gammas/s.Ci), VELM 23 Grp, 25 MWd/kgU, $18 \mathrm{~kW} / \mathrm{kgU}$, Continuous Operation $=1388.9 \mathrm{~d}$ 


\section{LIST OF TABLES (cont)}

Table

$\underline{\text { Page }}$

A.2 Gamma Source Spectra (gammas/s.Ci), VELM 23 Grp, 50 MWd/kgU,

$40 \mathrm{~kW} / \mathrm{kgU}$, Continuous Operation $=1250 \mathrm{~d}$

A.3 Gamma Source Spectra (gammas/s.Ci), VELM 23 Grp. HFED UA1,

Module 1, Slot 1, 40.24\% Enrichment, 94\% Depleted,

7.65E-4 MW/gU

A.4 Gamma Source Spectra (gammas/s.Ci), VELM 23 Grp. HFED U3O8,

Module 5, Slot 10, 93.21\% Enrichment, 80\% Depleted,

2.65E-3 MW/gU

A.5 Gamma Source Spectra (gammas/s.Ci), VELM 23 Grp. HFED U6Fe,

Module 23, Slot 6, 19.84\% Enrichment, 27\% Depleted,

5.59E-02 MW/gU

B.1 Light-Element Activity at 15-year Decay Period, $50 \mathrm{MWd} / \mathrm{kgU}, 40 \mathrm{~kW} / \mathrm{kgU}$,

U-wt\% (U234=.037, U235=4.200, U236=.019, U238=95.744); Continuous Operation $=1250 . \mathrm{d}$; Tbl A.4 ORNL-6698. Cycle 3 of 5. Clad is Zircaloy: Wt\% (97.91 Zr, 1.59 Sn, 0.5 Fe). SCALE 4.2 Composition Library. See Tbl. 3.11, ORNL-6698 for composition. ........................

B.2 Actinide Activity at 15-year Decay Period, $50 \mathrm{MWd} / \mathrm{kgU}, 40 \mathrm{~kW} / \mathrm{kgU}$, U-wt\%(U234=.037, U235=4.200, U236=.019,U238:=95.744); Continuous Operation $=1250 \mathrm{~d}$; Tbl A.4 ORNL-6698. Cycle 3 of 5. Clad is Zircaloy: Wt\% (97.91 Zr, 1.59 Sn, $0.5 \mathrm{Fe}$ ). SCALE 4.2 Composition Library. See Tbl 3.11, ORNL 6698 for composition. ........................... B-4

B.3 Fission Product Activity at 15-year Decay Period, $50 \mathrm{MWd} / \mathrm{kgU}, 40 \mathrm{~kW} / \mathrm{kgU}$, U-wt\%(U234=.037, U235=4.200, U236=.019, U238=95.744); Continuous Operation $=1250 \mathrm{~d}$; Tbl A.4 ORNL 6698. Cycle 3 of 5. Clad is Zircaloy: Wt\% (97.91 Zr, $1.59 \mathrm{Sn}, 0.5 \mathrm{Fe}$ ). SCALE 4.2 Composition Library. See Tbl 3.11, ORNL-6698 for composition. 


\section{ACKNOWLEDGEMENTS}

The authors would like to acknowledge the Waste Management and Remedial Action Division for the funding of this study, Douglas W. Turner for his patient support of this project, and to Robert N. Morris for his guidance in the areas to be analyzed. The contributions of James D. Drischler in the production of the major tables and Tamara R. Henson in the preparation of the final report are gratefully acknowledged. 
. 


\begin{abstract}
The Waste Management and Remedial Action Division has planned a modification sequence for storage facility 7827 in the Solid Waste Storage Area (SWSA). The modification cycle is as follows: 1) modify an empty caisson, 2) transfer the spent nuclear fuel (SNF) of an occupied caisson to a hot cell in building 3525 for inspection and possible repackaging, and 3) return the package to the modified caisson in the SWSA. Although the SNF to be moved is in the solid form, it has different levels of activity. Thus, the following five shipping casks will be available for the task: 1) the Loop Transport Carrier, 2) the In-Pile Loop LITR HB-2 Carrier, 3) the 6.5-Inch HRLEL Carrier, 4) the HFIR Hot Scrap Carrier, and 5) the 10-Inch ORR Experiment Removal Shield Cask. This report describes the shielding tasks for the five casks. These included the determination of the shielding characteristics, any streaming avenues, estimation of thermal limits, and shielding calculational uncertainty for use in the transportation plan.
\end{abstract}





\subsection{INTRODUCTION}

The Waste Management and Remedial Action Division has planned a modification sequence for storage facility 7827 in the Solid Waste Storage Area (SWSA). The modification cycle will be carried out in three steps: 1) modify an empty caisson, 2) transfer the spent nuclear fuel (SNF) of an occupied caisson to a hot cell in building 3525 for inspection and possible repackaging, and 3) return the package to the modified caisson in the SWSA.

Although the SNF to be moved is in the solid form, it has different levels of activity and is in different shapes geometrically. Thus, the following five shipping casks were made available for the task: 1) the Loop Transport Carrier, 2) the In-Pile Loop LITR (Low-Intensity Test Reactor) HB-2 Carrier, 3) the 6.5-Inch HRLEL (High-Radiation-Level Experimental Laboratory) Carrier, 4) the HFIR (High-Flux Isotope Reactor) Hot Scrap Cask, and 5) the 10-Inch ORR experiment Removal Shield Cask.

For the five shipping casks, this report describes shielding tasks, which are to determine: 1) the shielding characteristics, 2) any radiation streaming avenues from inside the casks to the external surface, 3 ) the thermal load for the casks and the source material, and 4) the shielding calculational uncertainty.

The activated material present in the SNF includes fuel, fission products, transuranic elements, and cladding activation products. Since there are many different radioisotopes present in the SNF, it was decided that the various sources chosen would be normalized to $1 \mathrm{Ci}$. Seven specific sources, which are representative of the myriad of sources present in the caissons, were chosen. These included ${ }^{60} \mathrm{Co},{ }^{137} \mathrm{Cs}$, two Light-Water-Reactor (LWR) MFP sources, representative of different burnups, and three Oak Ridge Reactor (ORR) MFP sources representative of different initial enrichments and final burnups.

Point isotopic generation/depletion calculations were made to determine the mixed fission-product photon (or gamma-ray) sources. The five three-dimensional casks were reduced to $(\mathrm{R}, \mathrm{Z})$ models, and a cross-section library was chosen. Two-dimensional radiation transport calculations were made using the geometry, source, and cross-section data to determine the doses at specific points on the surface and one meter from the surface of the casks. Plots and tables of the results are presented.

Due to the voluminous data generated in a shielding analysis of this type, most of the information used to construct the final tables of results are presented in the appendices. In particular, Appendix A presents the energy dependent photon source spectra as a function of time after burnup for the one mixed fission product source; Appendix B presents the constituent isotopes comprising the one mixed fission product source at fifteen years after burnup; Appendix $C$ presents graphical representations of the computer models used in the analysis of the five shipping casks; Appendix D presents the isodose contours for the various photon sources and casks analyzed in this report; and Appendix E presents the various code input files used in the analyses. 


\subsection{DESCRIPTION OF THE SHIPPING CASKS}

The SNF stored in the 7827 storage facility at the SWSA site takes on many shapes and sizes. Consequently, multiple shipping casks are required for transport of these waste canisters between the storage facility and the hot cells for inspection and/or repackaging. Below is a brief description of each of the five casks identified for the movement of the SNF.

Loop Transport Carrier The Loop Transport Carrier is a lead-filled steel cask approximately $61 \mathrm{~cm}$ in diameter, $225 \mathrm{~cm}$ long, and weighs $6890 \mathrm{~kg}$. The lead shielding is nominally $21 \mathrm{~cm}$ thick. The central cavity is a stainless steel lined cylinder approximately 14.5 $\mathrm{cm}$ in diameter and $175 \mathrm{~cm}$ long. Loading can be accomplished through either a sliding door on the bottom or a flanged gasketed plug on the top. The cask can be handled in either the normal horizontal position using the lifting ears or in a vertical position using the trunnions. The Loop Transport Carrier identification number is $8 \mathrm{~S} 16-80$, and the reference engineering drawing number is E-35362.

In-Pile Loop LITR HB-2 Carrier The In-Pile Loop LITR HB-2 Carrier is a lead filled stainless steel cask approximately $54 \mathrm{~cm}$ in diameter, $282 \mathrm{~cm}$ long, and weighs $6140 \mathrm{~kg}$. The lead shielding is nominally $15 \mathrm{~cm}$ thick. The cylindrical central cavity is approximately $21 \mathrm{~cm}$ in diameter, $243 \mathrm{~cm}$ long, and is lined with stainless steel (Type 347). Loading can be accomplished through a set of interlocking double doors on one end or a single sliding door on the other end. The cask can be handled in either the horizontal or vertical position using lifting eyes on the top or side. The In-Pile Loop LITR HB-2 Carrier identification number is $6 \mathrm{~S} 14-55$, and the reference engineering drawing number is E-23286.

This carrier is similar to the In-Pile Loop ORR-HN-1 Carrier (identification number 7S19-73 and reference engineering drawing number E-28066), and the LITR In-Pile Loop Carrier (identification number 6S13-54 and reference engineering drawing number E-16590). The major differences are that the ORR-HN-1 Carrier utilizes approximately $19 \mathrm{~cm}$ of lead shielding instead of the $15 \mathrm{~cm}$ on the LITR HB-2 Carrier, and the LITR In-Pile Carrier has a smaller central cavity size $(19 \mathrm{~cm}$ in diameter instead of the $21 \mathrm{~cm}$ on the LITR HB-2 Carrier).

6.5-Inch HRLEL Carrier The 6.5-Inch HRLEL Carrier is a lead filled cylindrical stainless steel cask approximately $58 \mathrm{~cm}$ in diameter and $130 \mathrm{~cm}$ long welded to a $61-\mathrm{cm}$-wide by $68-\mathrm{cm}$-deep by $70-\mathrm{cm}$-high lead filled stainless steel rectangular prism. The lead shielding is nominally $20 \mathrm{~cm}$ thick and the total cask weighs approximately $5900 \mathrm{~kg}$. The central cavity is a stainless steel lined cylinder approximately $18 \mathrm{~cm}$ in diameter and $102 \mathrm{~cm}$ long. Loading can be accomplished through either a petcock door located in the lower rectangular portion of the cask or a flanged plug on the cylindrical top portion of the cask. The cask can be handled using the trunnions either on the two ends or in the center along each side of the cask. The carrier can be sealed on both ends by installation of $2.54-\mathrm{cm}$-thick aluminum gasketed end plates. The 6.5-Inch. HRLEL Carrier identification number is 8S15-159, and the reference engineering drawing number is E-44010. 
HFIR Hot Scrap Shipping Cask The HFIR Hot Scrap Shipping Cask is a modification of the OD-2 LITR Fuel Element Carrier originally designed to be loaded from the top. The OD-2 cask was modified (and renamed) to be loaded from the bottom and used for transfers at the SWSA site. The Hot Scrap Cask is a lead-filled cylindrical stainless steel container approximately $80 \mathrm{~cm}$ in diameter, $149 \mathrm{~cm}$ in length, and weighs $6700 \mathrm{~kg}$. The cask sits on a rectangular base plate $122 \mathrm{~cm}$ by $132 \mathrm{~cm}$ and is designed to be transported vertically. The lead shielding is nominally $21-\mathrm{cm}$-thick, and the central cavity is a stainless-steel-lined cylinder $31.75 \mathrm{~cm}$ in diameter and $93 \mathrm{~cm}$ in length. A stainless steel basket approximately $30.50 \mathrm{~cm}$ in diameter and $87 \mathrm{~cm}$ in length can be inserted into the cavity for the transport of ORR type fuel elements. Loading can be accomplished through either a sliding door on the bottom or a flanged plug on the top. The cask is handled with two trunnions located on the sides 180 degrees apart. The HFIR Hot Scrap Shipping Cask identification number is 9S15-135, and the reference engineering drawing number is D-37780.

10-Inch ORR Experiment Removal Shield The 10-Inch ORR Experiment Removal Shield cask is a multisectional lead filled stainless steel cask composed of four segments. One segment is approximately $74 \mathrm{~cm}$ in diameter, $212 \mathrm{~cm}$ in length, and contains $20-\mathrm{cm}$-thick lead shielding. This segment (referred to as the heavy shielded segment) also includes a 20-cm-thick lead-filled stainless steel sliding door for loading the cask. There are two more segments, each approximately $63.5 \mathrm{~cm}$ in diameter and $122 \mathrm{~cm}$ in length, which contain 14.6-cm-thick lead shielding. These two segments are referred to as the light shielded segments. Finally, there is one more segment approximately $63.5 \mathrm{~cm}$ in diameter and $22 \mathrm{~cm}$ in length which contains 14.6-cm-thick lead shielding. This section also contains a 14.6-cm-thick lead-filled stainless steel sliding door and is referred to as the light shielded door assembly. In transport operations, this cask may be used in multiple configurations. However, for the purposes of the SNF transfer sequence, the cask will be bolted together in the longest configuration (i.e., the heavy section, two light sections, and the light door) resulting in a total cask weight of $16,750 \mathrm{~kg}$. In this configuration, the central cavity is a stainless steel lined cylinder approximately $27 \mathrm{~cm}$ in diameter and $430 \mathrm{~cm}$ in length. Loading access is accomplished through the heavy shielded and/or light shielded doors located on the two ends of the cask. Handling of the cask is accomplished through the use of six trunnions located along each side of the cask with the cask normally positioned in the horizontal position. The 10-Inch ORR Experiment Removal Shield identification number is 9S37-175, and the reference engineering drawing is D-25990. 


\subsection{DESCRIPTION OF THE CROSS SECTIONS}

The photon multi-energy-group (commonly called multigroup) transport cross-section set from the VELM ${ }^{1}$ 61-neutron, 23-photon library (VELM61) was used. The name of the library is an acronym for VITAMIN-E Liquid Metal (VELM). The neutron and photon energy group structure (Table 1 shows the photon group structure) is a subset of the VITAMIN-E ${ }^{2}$ group structure, and the cross sections were collapsed from the VITAMIN-E library. Although the libraries were specifically designed for sodium-cooled reactor shield analysis, the photon interaction data is general enough for most other purposes.

Table 1. VELM Photon Energy-Group Structure

\begin{tabular}{|c|c|c|c||}
\hline Group & $\begin{array}{c}\text { Top Energy } \\
(\mathrm{MeV})\end{array}$ & Group & $\begin{array}{c}\text { Top Energy } \\
(\mathrm{MeV})\end{array}$ \\
\hline 1 & $1.40 \mathrm{E} 7$ & 13 & $1.00 \mathrm{E} 6$ \\
\hline 2 & $1.00 \mathrm{E} 7$ & 14 & $7.00 \mathrm{E} 5$ \\
\hline 3 & $8.00 \mathrm{E} 6$ & 15 & $6.00 \mathrm{E} 5$ \\
\hline 4 & $7.50 \mathrm{E} 6$ & 16 & $5.10 \mathrm{E} 5$ \\
\hline 5 & $7.00 \mathrm{E} 6$ & 17 & $4.00 \mathrm{E} 5$ \\
\hline 6 & $6.00 \mathrm{E} 6$ & 18 & $3.00 \mathrm{E} 5$ \\
\hline 7 & $5.00 \mathrm{E} 6$ & 19 & $1.50 \mathrm{E} 5$ \\
\hline 8 & $4.00 \mathrm{E} 6$ & 20 & $1.00 \mathrm{E} 5$ \\
\hline 9 & $3.00 \mathrm{E} 6$ & 21 & $7.00 \mathrm{E} 4$ \\
\hline 10 & $2.50 \mathrm{E} 6$ & 22 & $4.50 \mathrm{E} 4$ \\
\hline 11 & $2.00 \mathrm{E} 6$ & 23 & $2.00 \mathrm{E} 4$ \\
\hline 12 & $1.50 \mathrm{E} 6$ & & $1.00 \mathrm{E} 4 *$ \\
\hline
\end{tabular}

* Bottom of energy-group 23.

The five shipping casks designated for the transport of the SNF are comprised of three basic materials: stainless steel, lead, and mild steel. While there were several different steels used in the construction of the casks, for the purposes of the shielding analyses, AISI Type 347 was chosen as the stainless steel, and SAE Type 1020 was chosen for the mild steel. The small differences in the elemental compositions of the other steels utilized in the casks' construction will cause negligible effects on the radiation transport analyses. The composition of the casks' materials used in the radiation shielding analysis were mixed using the GIP: Group-Organized Cross Section Input Program ${ }^{3}$ and are presented in Table 2. 
Table 2. Composition of Materials Used in the Radiation Shielding Analysis of the Spent Nuclear Fuel Shipping Casks.

\begin{tabular}{|c|c|c|c|c|c|}
\hline \multirow[b]{2}{*}{ Element } & \multicolumn{5}{|c|}{ Material Composition (atoms $/ \mathrm{barn} \cdot \mathrm{cm}$ ) } \\
\hline & $\begin{array}{l}\text { Dry } \\
\text { Air }\end{array}$ & $\begin{array}{c}\text { Steel } \\
\text { AISI } 347\end{array}$ & Lead & $\begin{array}{c}\text { Steel } \\
\text { SAE } 1020\end{array}$ & Aluminum \\
\hline $\begin{array}{l}\text { Carbon } \\
\text { Nitrogen } \\
\text { Oxygen } \\
\text { Aluminum } \\
\text { Silicon } \\
\text { Argon } \\
\text { Chromium } \\
\text { Manganese } \\
\text { Iron } \\
\text { Nickel } \\
\text { Lead }\end{array}$ & $\begin{array}{l}3.98 \mathrm{E}-05^{2} \\
1.07 \mathrm{E}-05 \\
2.37 \mathrm{E}-07\end{array}$ & $\begin{array}{l}3.19 \mathrm{E}-04 \\
\\
1.71 \mathrm{E}-03 \\
1.66 \mathrm{E}-02 \\
1.74 \mathrm{E}-03 \\
5.91 \mathrm{E}-02 \\
8.16 \mathrm{E}-03\end{array}$ & $3.30 \mathrm{E}-02$ & $\begin{array}{l}\text { 4.21E-04 } \\
\text { 3.88E-04 } \\
8.40 \mathrm{E}-02\end{array}$ & $6.03 \mathrm{E}-02$ \\
\hline$\rho\left(\mathrm{g} / \mathrm{cm}^{3}\right)$ & $1.23 \mathrm{E}-03$ & $7.95 \mathrm{E}+00$ & $1.13 E \dashv-01$ & $7.86 \mathrm{E}+00$ & $2.70 \mathrm{E}+00$ \\
\hline
\end{tabular}

${ }^{2}$ Read as $3.98 \times 10^{-5}$.

Elemental KERMA (Kinetic Energy Released in Material) factors, used to determine the heating rates, were pulled from the VELM library using the PAL module of the AMPX ${ }^{4}$ code system. The elements making up the major materials of the casks were $\mathrm{C}, \mathrm{Al}, \mathrm{Si}, \mathrm{Cr}$, $\mathrm{Mn}, \mathrm{Fe}, \mathrm{Ni}$, and $\mathrm{Pb}$.

The KERMA factors from PAL were then processed through the AMP ${ }^{5}$ code to change the units from (MeV.b)/(photon.atom) to (W.s)/(photon.cm), and to place the KERMA data in cross-section format.

The cross-section data for the point isotopic generation/depletion calculations were taken from the libraries ${ }^{6}$ produced in support for major revisions to the current Nuclear Regulatory Commission decay heat rate guide. 


\subsection{DESCRIPTION OF THE RADIATION SOURCES}

Table 3 contains those radioisotopes which form the bulk of the activity in the SNF (from the SNF Database ${ }^{7}$ ). After all the photons released from either each of these or from their daughters had been examined, it was decided to use the ${ }^{60} \mathrm{Co},{ }^{137} \mathrm{Cs}$, and the MFP as the major sources in the calculations.

Table 3. Radioisotopes Forming Bulk of SNF

\begin{tabular}{|c|c||}
\hline $\begin{array}{c}\text { Radioisotope } \\
\text { MFP }\end{array}$ & 10,000 \\
\hline${ }^{60} \mathrm{Co}$ & 113,000 \\
\hline${ }^{90} \mathrm{Sr}$ & 70,000 \\
\hline${ }^{137} \mathrm{Cs}$ & 3,800 \\
\hline${ }^{151} \mathrm{Sm}$ & 1,200 \\
\hline${ }^{152} \mathrm{Eu}$ & 200 \\
\hline${ }^{154} \mathrm{Eu}$ & 16,000 \\
\hline${ }^{155} \mathrm{Eu}$ & 10 \\
\hline${ }^{226} \mathrm{Ra}$ & 1 \\
\hline${ }^{232} \mathrm{Th}$ & $<<1$ \\
\hline${ }^{233} \mathrm{U}$ & $<1$ \\
\hline${ }^{235} \mathrm{U}$ & $<<1$ \\
\hline${ }^{237} \mathrm{~Np}$ & $<<1$ \\
\hline${ }^{238} \mathrm{U}$ & $<<1$ \\
\hline${ }^{239} \mathrm{Pu}$ & 16 \\
\hline${ }^{240} \mathrm{Pu}$ & 29 \\
\hline${ }^{241} \mathrm{Pu}$ & 1,344 \\
\hline${ }^{241} \mathrm{Am}$ & 2 \\
\hline${ }^{244} \mathrm{Cm}$ & $<<1$ \\
\hline${ }^{252} \mathrm{Cf}$ & $<<1$ \\
\hline
\end{tabular}


Seven primary photon sources were developed for the casks radiation transport calculations. These sources were all normalized to $1 \mathrm{Ci}$. Two of the sources were ${ }^{60} \mathrm{Co}$ and ${ }^{137} \mathrm{Cs}$; and, because of the known photon lines, no depletion code calculation was necessary to determine the number of photons emitted per curie. [NOTE: The source strength for ${ }^{137} \mathrm{Cs}$ required an adjustment to conserve energy because the midpoint of the VELM61 library energy bin is $0.65 \mathrm{MeV}$ and the energy of the ${ }^{137} \mathrm{Cs}$ emitted photon is $0.662 \mathrm{MeV}$ with an associated yield of $92 \%$. Therefore, to get the proper normalization, the number of photons per curie is multiplied by the yield, and the ratio of the photon emitted energy divided by the VELM61 energy group average energy.] Table 4 shows the source strength and energy group for these two sources.

Table 4. Energy Group and Source Strength for ${ }^{60} \mathrm{Co}$ and ${ }^{137} \mathrm{Cs}$

\begin{tabular}{|c|c|c|}
\hline Source & Energy Group & Strength (Photons/Ci) \\
\hline \hline${ }^{60} \mathrm{Co}$ & 12 & $7.400 \mathrm{E}+10$ \\
\hline${ }^{137} \mathrm{Cs}$ & 14 & $3.467 \mathrm{E}+10$ \\
\hline
\end{tabular}

The other five photon sources resulted from mixed-fission products (MFP) and required point isotopic generation/depletion calculations be made with the ORIGEN-S ${ }^{8}$ code.

Two of the five MFP photon sources were generated from Pressurized Water Reactor (PWR) data taken from Tables 3.11 and A.4 of Ref. 5. One source was generated from a burnup of 25 megawatt days per kilogram of uranium (2.4\% enrichment), to be noted symbolically as $25 \mathrm{MWd} / \mathrm{kgU}$ and a specific power of $18 \mathrm{~kW} / \mathrm{kgU}$; the second source was generated from a burnup of $50 \mathrm{MWd} / \mathrm{kgU}$ ( $4.2 \%$ enrichment) and a specific power of $40 \mathrm{~kW} / \mathrm{kgU}$.

The final three MFP photon sources were generated from three representative miniature fuel plates irradiated during the Department of Energy's (DOE) Reduced Enrichment Research and Test Reactor ${ }^{9}$ (RERTR) Progran which began in 1978. The fuel plates were irradiated in a special test facility, designated as High-Uranium-Loaded Fuel Element Development (HFED), in the ORR core during the period of 1980 through 1987. The pertinent attributes of the miniplates were taken from Ref. 9 and are listed in Table 5.

Table 5. Three RERTR Program Miniplate Attributes

\begin{tabular}{||c|c|c|c|c|c|c||}
\hline $\begin{array}{c}\text { Module } \\
\text { No. }\end{array}$ & $\begin{array}{c}\text { Slot } \\
\text { No. }\end{array}$ & $\begin{array}{c}\text { Fuel } \\
\text { Type }\end{array}$ & $\begin{array}{c}\text { Enrichment } \\
\left(\mathrm{Wt}^{235} \mathrm{U}\right)\end{array}$ & $\begin{array}{c}\mathrm{U} \\
\text { Loading } \\
(\mathrm{g})\end{array}$ & $\begin{array}{c}\text { Irradiation } \\
\text { Time } \\
(\mathrm{FPD})\end{array}$ & $\begin{array}{c}{ }^{235} \mathrm{U} \\
\text { Depletion } \\
\text { (At. \%) }\end{array}$ \\
\hline 1 & 1 & $\mathrm{UAl}_{\mathrm{x}}$ & 40.24 & 5.470 & 470 & 94 \\
\hline 5 & 10 & $\mathrm{U}_{3} \mathrm{O}_{8}$ & 93.21 & 1.553 & 268 & 80 \\
\hline 23 & 6 & $\mathrm{U}_{6} \mathrm{Fe}$ & 19.84 & 18.390 & 91 & 27 \\
\hline
\end{tabular}

FPD $=$ Full Power Days 
The ORIGEN-S cross-section data were also taken from libraries generated during the production of the work reported in Ref. 6. Although this data only applied to the PWRs, several test calculations indicated that it could also be applied to the ORR miniplates with little error. This was because the photon sources were being normalized to $1 \mathrm{Ci}$, and the spectra for the out years ( 15 years and beyond) did not show large changes when normalized in this manner.

The energy-dependent photon source spectra as a function of time after burnup for one of the five MFP sources are presented in Appendix A, and the constituent isotopes comprising one MFP source at fifteen years after burnup are given in Appendix B. 


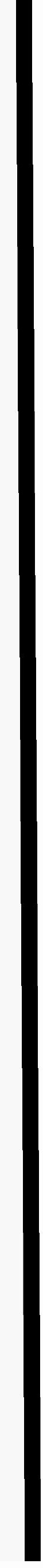




\subsection{DESCRIPTION OF CALCULATIONAL MODELS}

The engineering reference drawings for the five SNF casks described in Section 2.0 were utilized to design cylindrical $\mathrm{r}-\mathrm{z}$ models of the casks for analysis in the DORT ${ }^{10}$ two-dimensional radiation transport code system. Any engineering details which might contribute to a radiation streaming path were included in the calculational models to analyze their effect. Typical radial and axial mesh sizes varied from 0.5 to $1.0 \mathrm{~cm}$ in thickness resulting in transport models with between 43 and 64 radial intervals and between 243 and 641 axial intervals depending on the size of the cask. The casks models utilized a 240 direction symmetric quadrature set, a $\mathrm{P}_{5}$ Legendre expansion of the cross sections, and the VELM61 [84 energy group (61 neutron, 23 gamma)] cross-section library. The materials listed in Table 2 were mapped to the radial and axial mesh to generate the DORT calculational model. The source regions for each cask were modeled as air in the DORT calculations due to the lack of information on the material composition and physical description of the SNF canisters to be transported. Detailed graphical representations of the DORT calculational models are shown in Appendix C. Expanded views of the loading doors, and end plugs are also included in Appendix $C$ to indicate the detail associated with the calculational models. Penetrations in the sides of the shipping casks (e.g., view ports, light windows, etc.), were not included in the transport calculation models because, for the movement of the SNF, they would be plugged with lead-filled plugs of thicknesses equivalent to the cask wall thickness and would not result in measurable radiation streaming paths. 


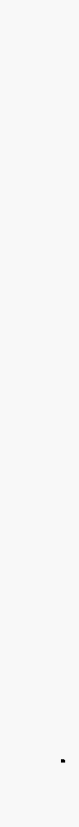




\subsection{DESCRIPTION OF CALCULATIONAL METHOD}

The flow diagram for the SNF shipping casks shielding analyses is given in Figure 1. The generation of the cross-section data for the ORIGEN-S and DORT codes was discussed in Section 3.0 and the calculation of the SNF energy dependent source spectra for input into DORT was discussed in Section 4.0.

The principal workhorse in the calculational sequence presented in Figure 1 is the DORT two-dimensional discrete-ordinates radiation transport code. DORT is primarily designed to solve large neutron and photon transport problems using the method of discrete ordinates. Most DORT problems deal with the calculation of radiation resulting from a given extraneous source, i.e., "fixed-source" problems. If such a system has fissile material and is subcritical, the multiplication can be calculated. The code also has $\mathrm{K}_{\text {eff }}$ capability and various types of searches. The directional quadrature set can be chosen from an arbitrary number of input sets. The choice can vary with spatial option and with energy group. This flexibility has proven quite effective in concentrating computer effort in areas needing attention, such as streaming gaps. Biased direction sets can be used when streaming is primarily in the upward/downward/horizontal directions. A variety of options allow sources to be specified at internal or external boundaries, distributed by space and energy, or determined from an input fluence guess. "First-collision-source" data (actually an analytical first-flight scattering source) can be accommodated. Output files for an "analytical-last-flight" integration can be obtained. These features provide increased accuracy when dealing with out-of-system or localized sources or detector locations. Both one-dimensional (1-D) and 2-D geometries can be treated. Discrete-ordinates geometries include 1-D plane or slab and 2-D X-Z, R-Theta, or R-Z. A powerful slab reflection/transmission feature is available. A variety of acceleration options are available. Extensive use of input options and output edits give the user very direct control over the iteration process. While this places a burden of decision-making on the user, it is essential to the solution of large and difficult problems. Default and recommended values assist the uninitiated in solving problems without much prior use of the code. Output source information to be used in coupling to other problems can be obtained.

Utilizing the macroscopic cross-section data generated in GIP, the SNF energy dependent source spectra generated in ORIGEN-S, and the calculational model information discussed in Section 5.0, the DORT code was executed to generate the requisite output files for the shielding analyses of the shipping casks. In particular, DORT generates a scalar flux file which is reduced to the more manageable Legendre $P_{0}$ flux component using the utility program RTFLUM: A Module For Converting, Expanding, And Editing Standard Data Files. ${ }^{3}$ This flux file is utilized in the ISOPLOT module of the DOGS ${ }^{11}$ code system to generate isodose contours for the shipping casks. Analysis of these isodose contours indicate potential streaming paths, dose levels within the shipping casks, and areas of maximum dose. DORT also generates a distributed source file for input into the FALSTF ${ }^{12}$ code system. FAISTF uses an analytical-last-flight integration technique to obtain the dose at selected detector locations on the surface of the shipping casks and at one meter from the surface of the casks. In both the ISOPLOT and FALSTF codes, the ANSI ${ }^{13}$ dose response function is used to determine the calculated dose rates per curie of source. Finally, DORT utilizes the KERMA factor data to generate the gamma-ray heating rates for the various SNF source/shipping cask combinations. Appendix E presents the listings of the various code input files used in the analyses of the different shipping cask. 


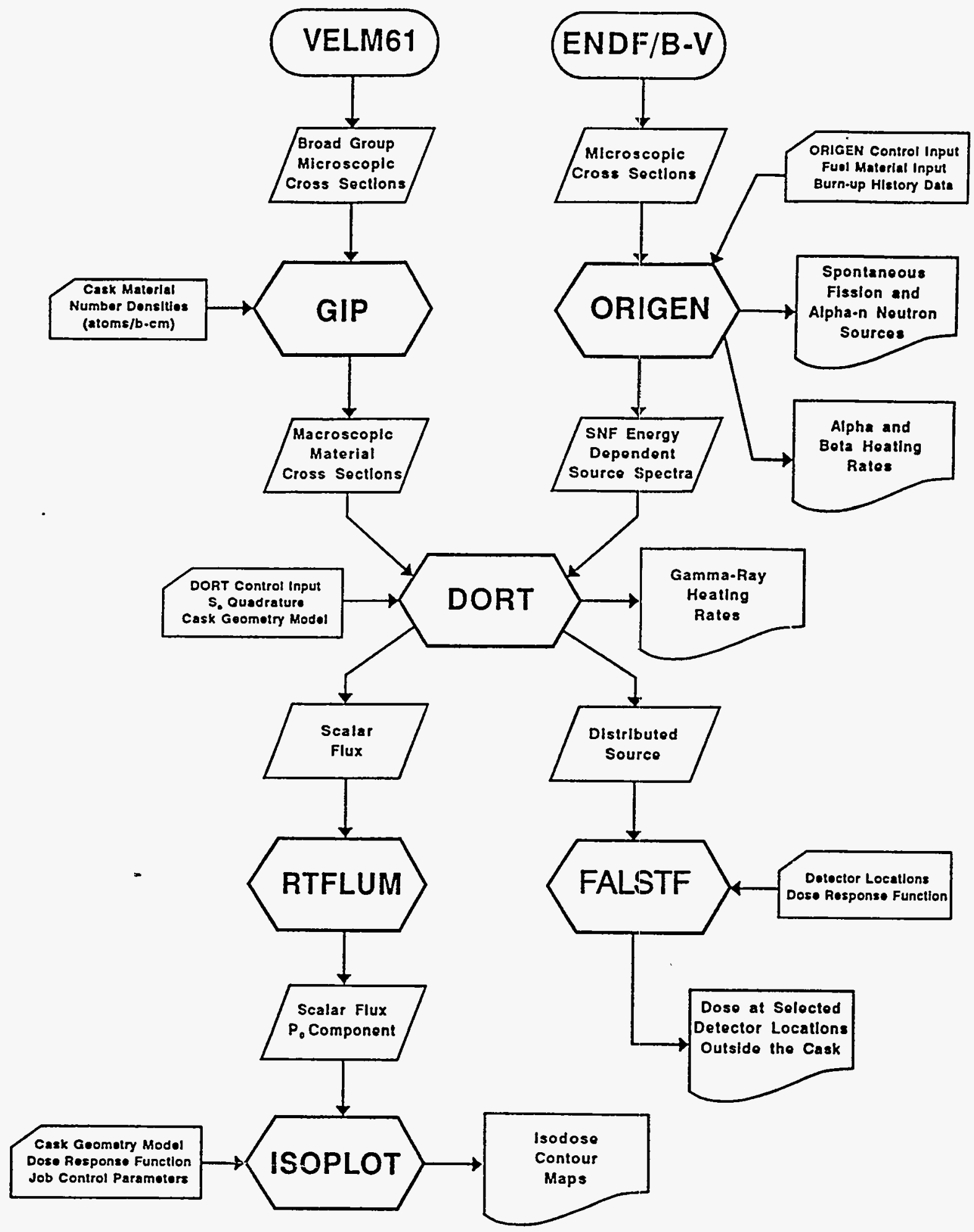

Figure 1. Flow Diagram of the SNF Shipping Casks Shielding Analysis. 


\subsection{RESULTS}

Two of the goals associated with this shielding analysis were to determine 1) the shielding characteristics and any radiation streaming paths from inside the casks to the external surface, and 2) the thermal load for the casks and the source material. With respect to these two goals, the transportation plan specifies a dose rate of $200 \mathrm{mrem} / \mathrm{hour}$ on the cask surface and $10 \mathrm{mrem} / \mathrm{hour}$ at one meter from the cask surface. With respect to the thermal load, the transportation plan specifies a nominal limit of $1 \mathrm{kw}$ total energy in the cask and source material.

To determine the total nuclear heating from the cask and the spent nuclear fuel materials within the cask, the contributions from alpha, beta, and photons had to be calculated. The alpha and beta heating rates were obtained from the ORIGEN-S calculations and are given in Table 6. Since ORIGEN-S employs a point isotopic generation/depletion calculation, these heating rates are geometry independent and a function of the source only. The photon heating rates were calculated using the DORT transport code and are given in Table 7. Because DORT performs a full radiation transport calculation and utilizes information about both the source and geometry, the photon (or gamma-ray) heating rates are calculated for each source/cask combination. In viewing the results presented in Table 7 , it is apparent that there is virtually no sensitivity to the cask geometry since the photon heating rates for a given source are within one percent of each other. An average value for a given source could be applied to all of the five casks without introducing significant error.

Table 6. Alpha and Beta Heating Rates Per Curie (W/Ci) by Source Type

\begin{tabular}{|c|c|}
\hline Source & Heating Rate (W/Ci) \\
\hline Co-60 & $5.77 \mathrm{E}-4$ \\
\hline $18 \mathrm{~kW} / \mathrm{kgU}, 25 \mathrm{MWd} / \mathrm{kgU}$ & $2.36 \mathrm{E}-3$ \\
\hline $40 \mathrm{~kW} / \mathrm{kgU}, 50 \mathrm{MWd} / \mathrm{kgU}$ & $2.70 \mathrm{E}-3$ \\
\hline $\mathrm{Cs}-137$ & $1.02 \mathrm{E}-3$ \\
\hline ORR MFP, $20 \%^{235} \mathrm{U}$ & $2.06 \mathrm{E}-3$ \\
\hline ORR MFP, $40 \%^{235} \mathrm{U}$ & $3.33 \mathrm{E}-3$ \\
\hline ORR MFP, $93 \%^{235} \mathrm{U}$ & $2.75 \mathrm{E}-3$ \\
\hline
\end{tabular}

To determine the shielding characteristics and any radiation streaming paths from inside the casks to the external surface, the DORT scalar fluxes were folded with the ANSI dose response function and plotted using the ISOPLOT module of the DOGS code system. These isodose contours are presented in Appendix $D$ for each of the different shipping-cask/SNF-source combinations. Visual analyses of these isodose contour plots indicate the complete dose profile for the cask, the calculated dose levels on the surface of the cask, and the potential streaming paths or areas of maximum dose on the surface of the 
Table 7. Gamma-Ray Heating Rates for the Designated Spent Nuclear Fuel Shipping Cask to be Used

to Transfer Radioactive Waste from the SWSA Area Sites, Building 7827 to Building 3525 and for Representative Sources Stored in the SWSA Area Sites, Building 7827.

\begin{tabular}{|c|c|c|c|c|c|c|c|}
\hline \multirow{2}{*}{$\begin{array}{c}\text { Designated } \\
\text { Spent Nuclear Fuel } \\
\text { Shipping Cask }\end{array}$} & \multicolumn{7}{|c|}{ Gamma-Ray Heating Rates per One Curie of Source } \\
\hline & ${ }^{60} \mathrm{Co}$ & $\begin{array}{c}\text { LWR MFP } \\
25 \mathrm{MWd} / \mathrm{kgU}\end{array}$ & $\begin{array}{c}\text { LWR MFP } \\
50 \mathrm{MWd} / \mathrm{kgU}\end{array}$ & ${ }^{137} \mathrm{Cs}$ & $\begin{array}{l}\text { ORR MFP } \\
20 \%{ }^{235} \mathrm{U}\end{array}$ & $\begin{array}{l}\text { ORR MFP } \\
40 \%{ }^{235} U\end{array}$ & $\begin{array}{l}\text { ORR MFP } \\
93 x^{235} \mathrm{U}\end{array}$ \\
\hline Loop Transport Carrier & $1.47000 \mathrm{E}-02$ & $8.55782 \mathrm{E}-04$ & $9.18118 \mathrm{E}-04$ & $3.56270 \mathrm{E}-03$ & $9.39623 \mathrm{E}-04$ & $1.05281 \mathrm{E}-03$ & $1.05380 \mathrm{E}-03$ \\
\hline LITR HB-2 In-Pile Loop Carrier & $1.46908 \mathrm{E}-02$ & $8.55170 \mathrm{E}-04$ & 9.17421E-04 & $3.56028 \mathrm{E}-03$ & $9.38938 \mathrm{E}-04$ & $1.05203 \mathrm{E}-03$ & $1.05304 \mathrm{E}-03$ \\
\hline 6.5 Inch HRLEL Carrier & $1.47477 \mathrm{E}-02$ & 8.60125E-04 & 9.22754E-04 & $3.57809 \mathrm{E}-03$ & $9.44663 \mathrm{E}-04$ & $1.05832 \mathrm{E}-03$ & $1.05939 \mathrm{E}-03$ \\
\hline HFIR Hot Scrap Carrier & $1.47426 \mathrm{E}-02$ & 8.59807E-04 & $9.22440 \mathrm{E}-04$ & $3.57761 \mathrm{E}-03$ & $9.44266 \mathrm{E}-04$ & $1.05791 \mathrm{E}-03$ & $1.05898 \mathrm{E}-03$ \\
\hline 10 Inch Expt. Removal Shield-ORR & 1.47801E-02 & $8.63336 \mathrm{E}-04$ & $9.26218 \mathrm{E}-04$ & 3.59191E-03 & 9.48283E-04 & $1.06229 \mathrm{E}-03$ & $1.06229 \mathrm{E}-03$ \\
\hline
\end{tabular}

${ }^{8}$ Gamma-Ray heating rate units are (watts)/(curie). 
cask. Typically, the results show weaknesses in the cask shielding in the areas of the end plugs and loading doors 2 especially if there is a large cross-sectional area of stainless steel (see dose contours for the Loop Transport Carrier End Plug in Figure D-3). These areas indicate where the maximum external surface and $1 \mathrm{~m}$-distance dose rates will probably occur. The isodose contours also indicate the relative magnitude (per curie) of the dose rates within the casks. One cask worth noting in particular, is the 10-Inch ORR Experiment Removal Cask (Figures D-39 to D-50). As stated in Section 2.0, this cask is a multisectional cask which can be bolted together in different combinations to accommodate different SNF transfers. The isodose contours in Appendix D indicate the stainless steel interface flanges where the various sections are bolted together present the areas of maximum dose and could be potential streaming paths.

Visual analysis of the isodose contours in Appendix D determined where to calculate the dose rates on the surface and at one meter distance from the surface of the cask in the FALSTF code. For each shipping cask, 34 different detector locations were chosen. Detectors $1-17$ are located on the surface of the casks (technically $3.81 \mathrm{~cm}$ from the surface), and detectors $18-34$ are located at 1-m distance from the surface. Graphical displays of the detector locations are given in Figures 2 through 6 for the five different shipping casks. The dose results for these detector locations are given in Tables 8 through 12 for each of the five shipping casks and all seven sources analyzed. The isodose contour results in Appendix D and the FALSTF results in Tables 8 - 12 are presented on a per-curie basis so that these data can be readily applied to the myriad of sources at the storage facility 7827 in the SWSA. Typically, for a given cask, the maximum dose rate occurred at the same detector position regardless of the SNF source. Although the absolute magnitude of the dose rate changed, the location at which it occurred remained constant. The major exception to this result is the ${ }^{137} \mathrm{Cs}$ results which are governed by a lower average energy gamma-ray than the ${ }^{60} \mathrm{Co}$ or various MFP sources. Also, the location of the maximum dose rate $1 \mathrm{~m}$ from the surface did not always correspond to the detector location in the general vicinity of the maximum dose rate on the cask surface. The maximum surface dose rates appear to be more affected by a local perturbation in the cask shielding (i.e., end plug, stainless steel spacer, etc.), whereas the maximum dose rate at one meter from the surface appears to be more affected by the geometric and material attenuation of the shipping cask relative to the detector location.

Figure 7, which shows the activity per gram of uranium of the MFP sources as a function of decay time, was created to aid in determining the MFP curie strength after irradiation. Rapid decay occurs in the first five years after irradiation. The actual source activity ( $\mathrm{Ci}$ ) for any of the five MFP sources can be determined after 5-40 years of decay if the decay time and the initial mass of uranium is known in the following way: the MFP source is chosen; the activity per initial gram of uranium is read from the figure using the chosen MFP source and the decay time; and the initial loading (grams of uranium) is multiplied by the activity per initial gram of uranium. 


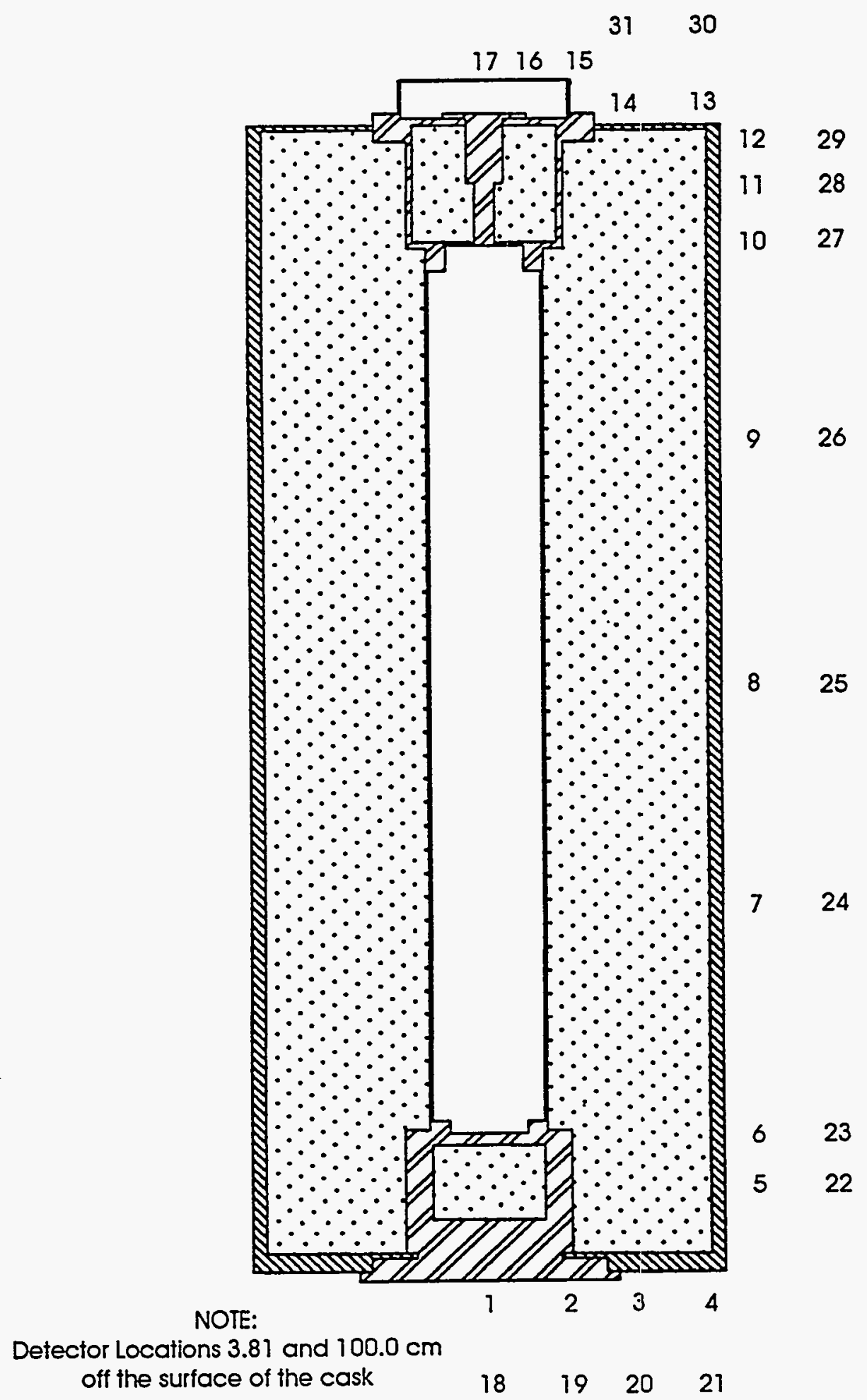

Figure 2. Detector Locations for the Surface and One Meter Distance Dose Rates Used in the Loop Transport Carrier SNF Shipping Cask Shielding Analysis. 


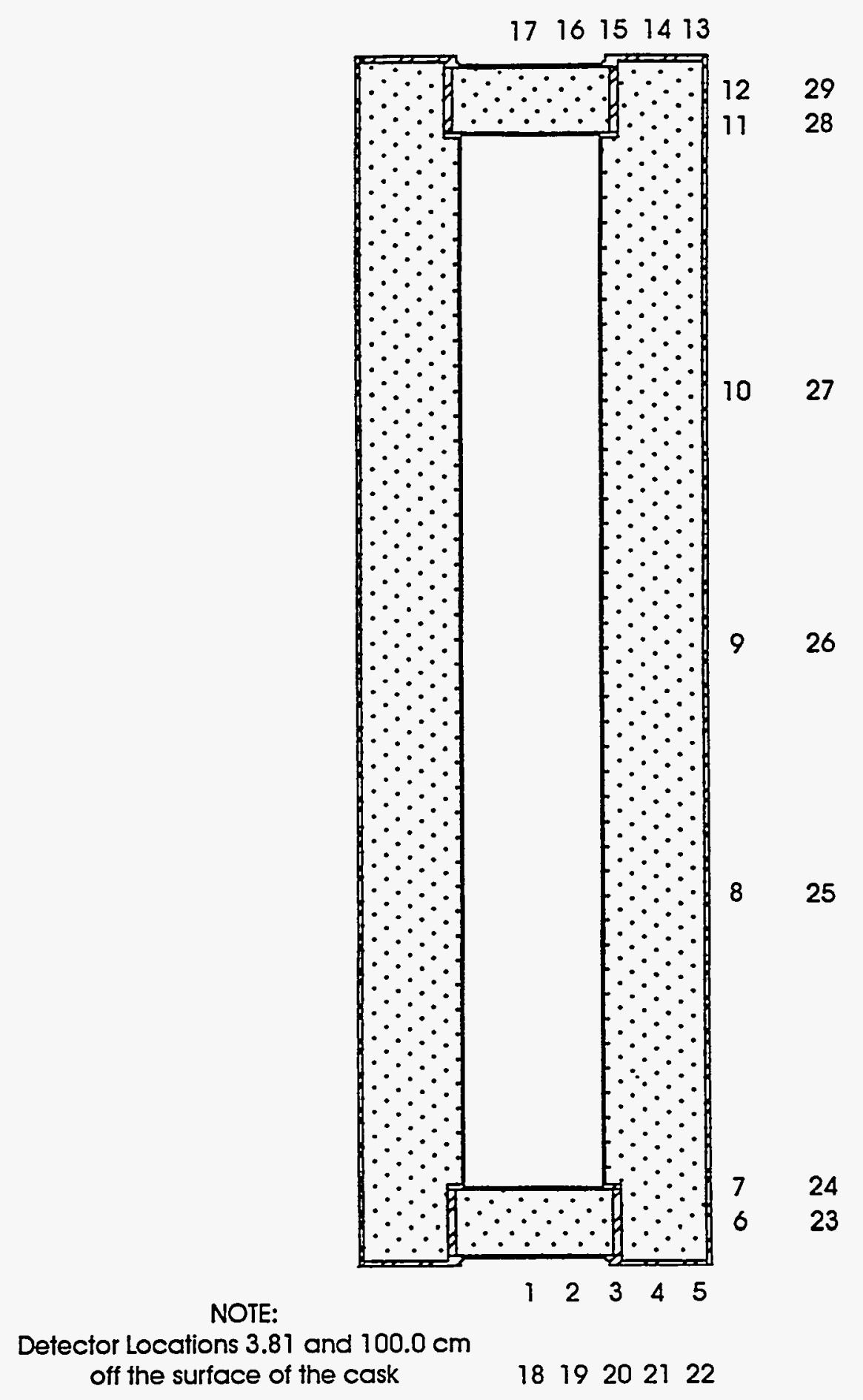

Figure 3. Detector Locations for the Surface and One Meter Distance Dose Rates Used in the In-Pile Loop LITR HB-2 Carrier SNF Shipping Cask Shielding Analysis. 


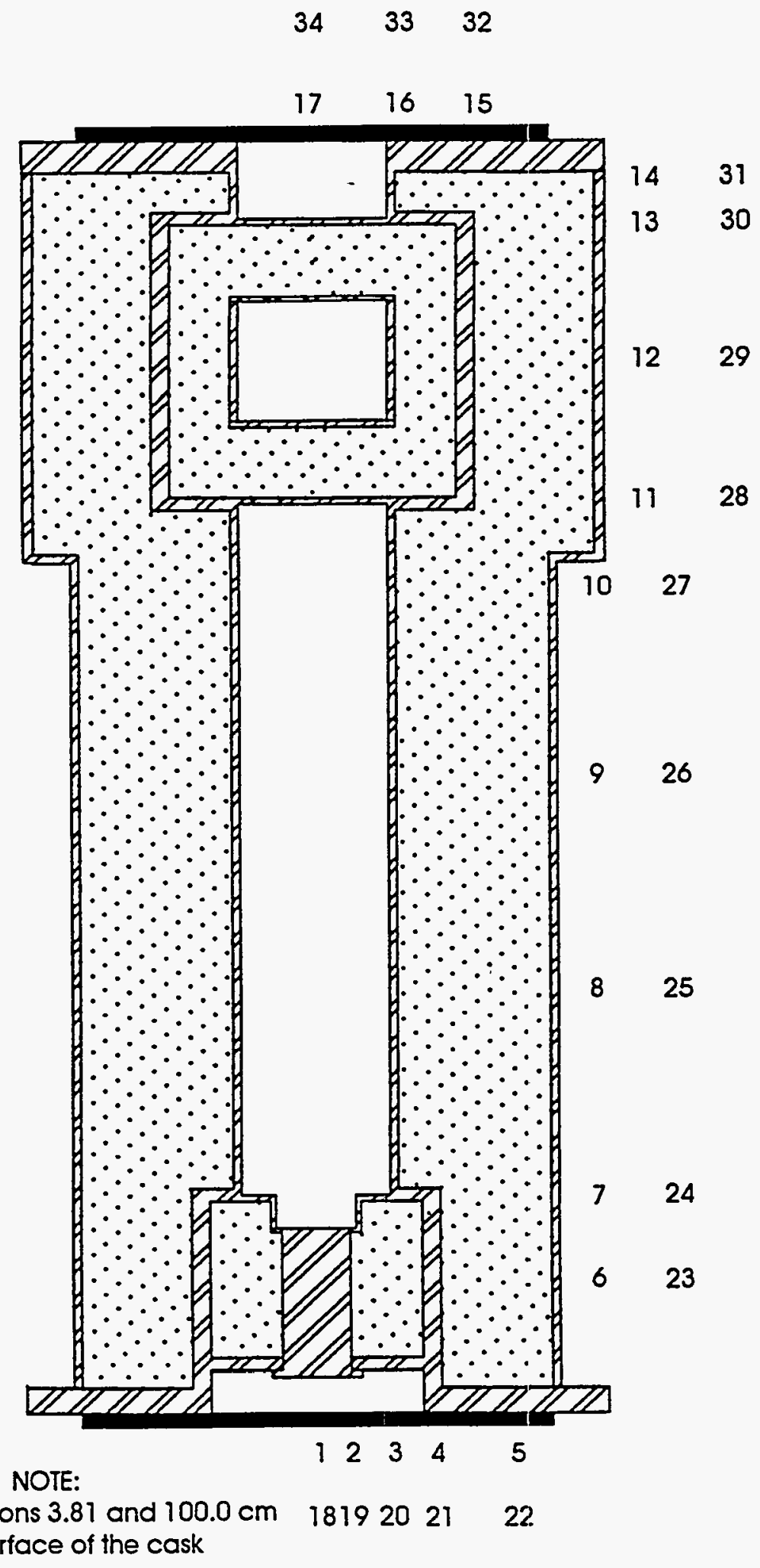

Figure 4. Detector Locations for the Surface and One Meter Distance Dose Rates Used in the 6.5 Inch HRIEL Carrier SNF Shipping Cask Shielding Analysis. 


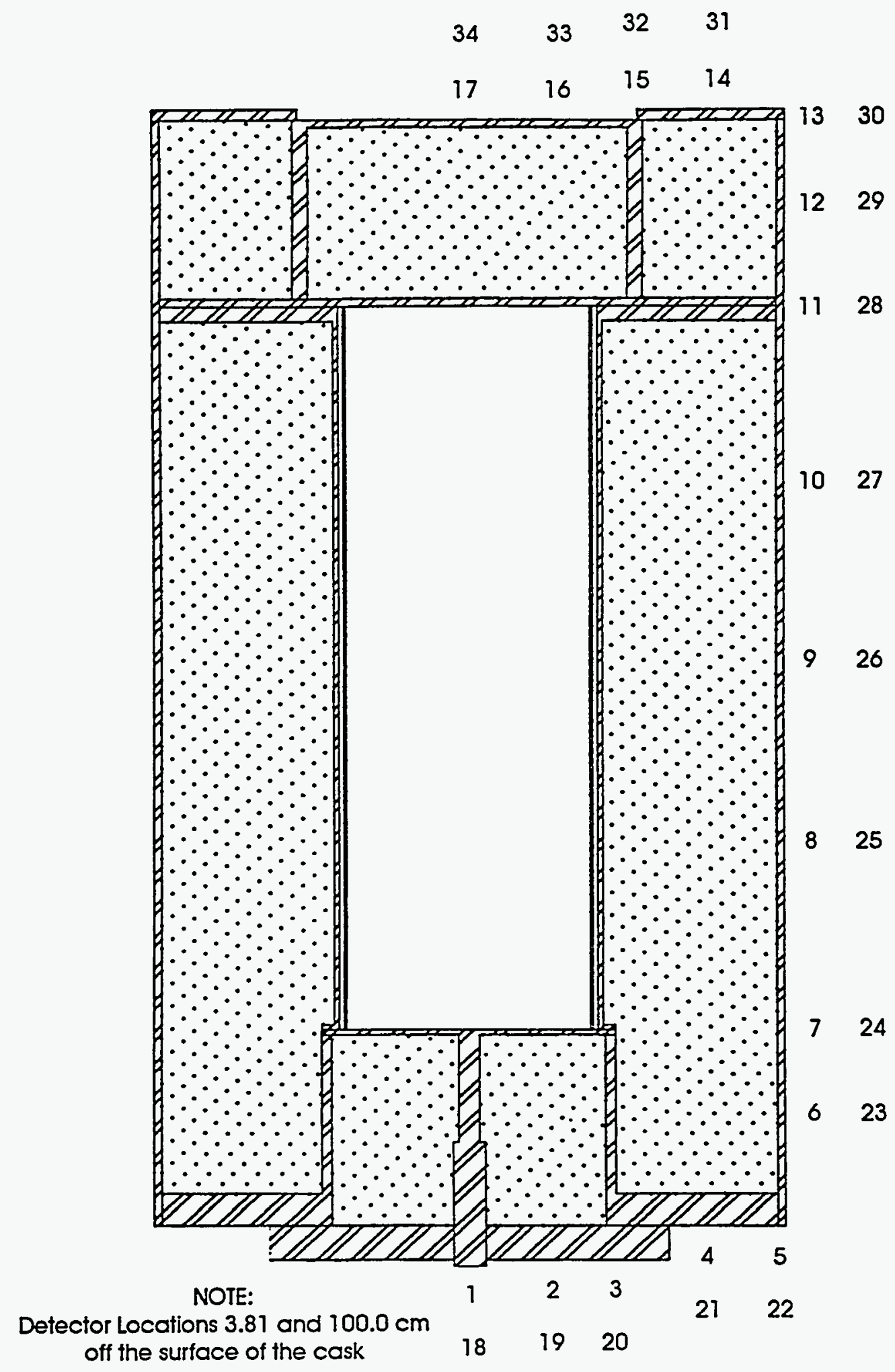

Figure 5. Detector Locations for the Surface and One Meter Distance Dose Rates Used in the HIFR Hot Scrap Carrier SNF Shipping Cask Shielding Analysis. 


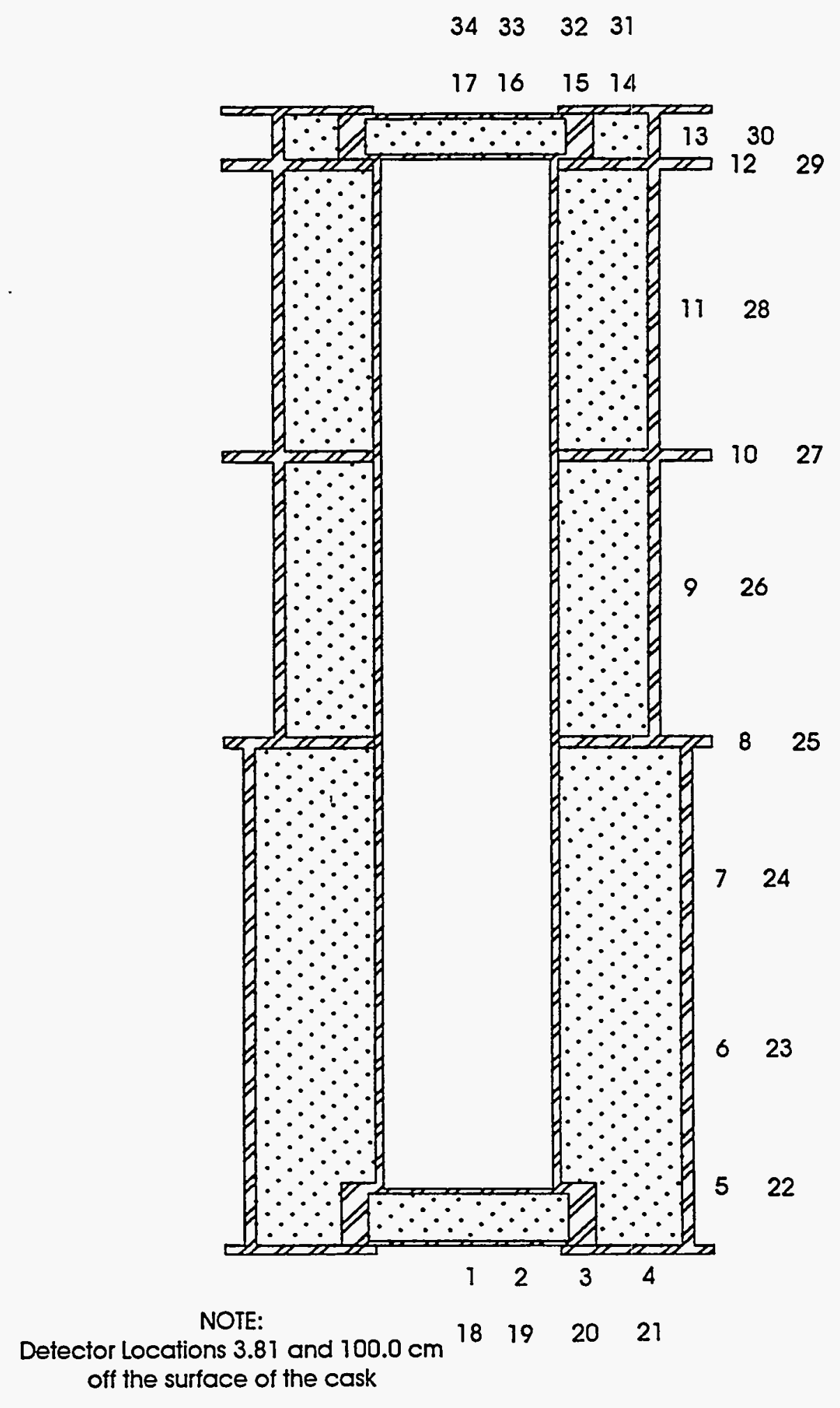

Figure 6. Detector Locations for the Surface and One Meter Distance Dose Rates Used in the 10 Inch ORR Experiment Removal Shield SNF Shipping Cask Shielding Analysis. 


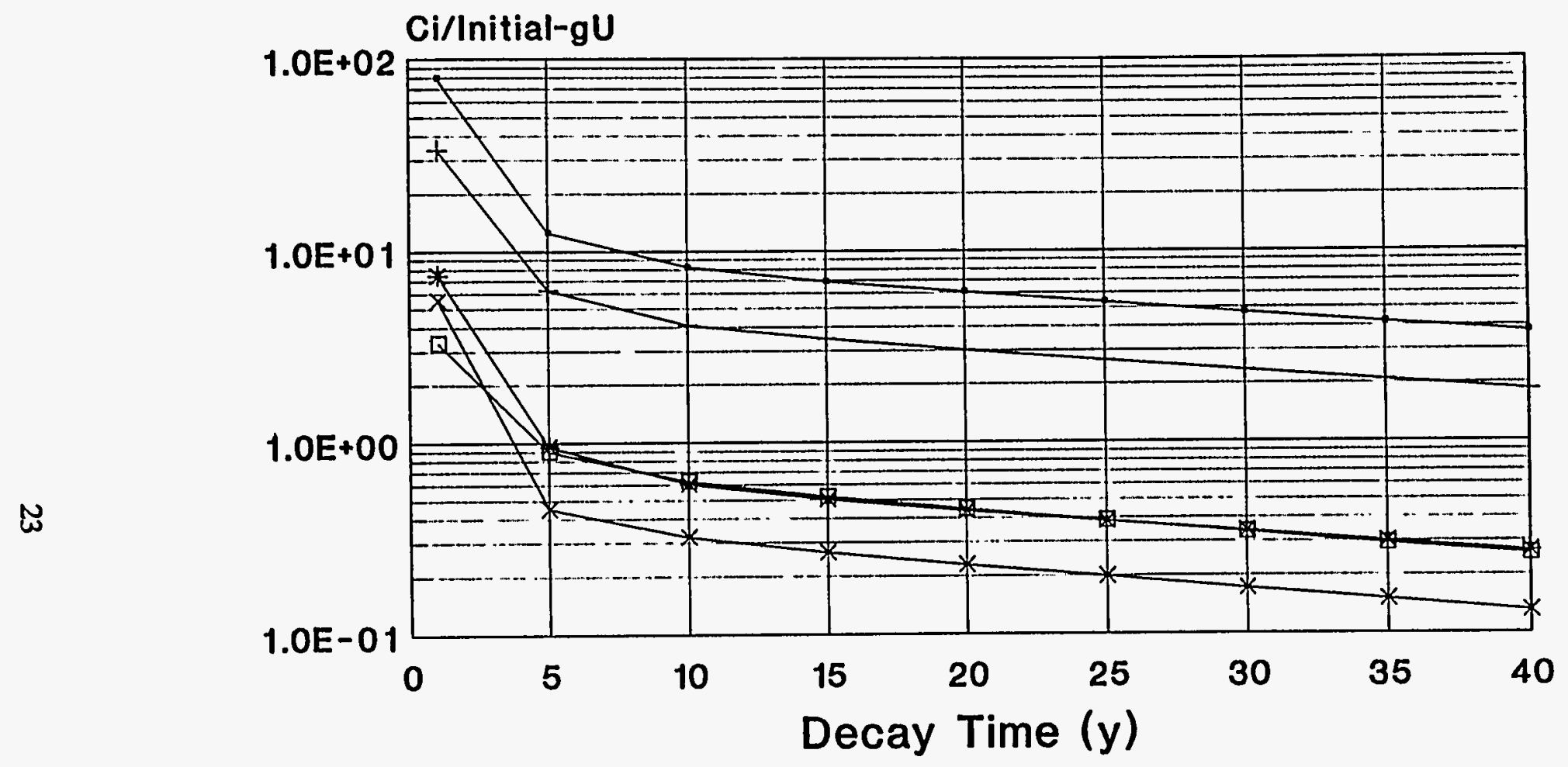

$\rightarrow$ ORR U3OB, 93\% $\rightarrow$ ORR UAl, 40\% $\rightarrow$ ORR U6Fe, 20\%

๑- PWR 50BU, 4.2\% $\rightarrow$ PWR 25BU, 2.4\%

\%-Enrichment; BU = Burnup (MWd/kgU)

Figure 7. Activity Per Initial Gram of Uranium Loading Versus Decay Time for the Five MFP Sources. 
Table 8. Gamma-Ray Dose Rates at Selected Detector Positions $3.81 \mathrm{~cm}$ and $1 \mathrm{~m}$ Away From the Surface of the Loop Transport Carrier Spent Nuclear Fuel Shipping Cask for Representative Sources Stored in the SWSA Area Sites, Building 7827.

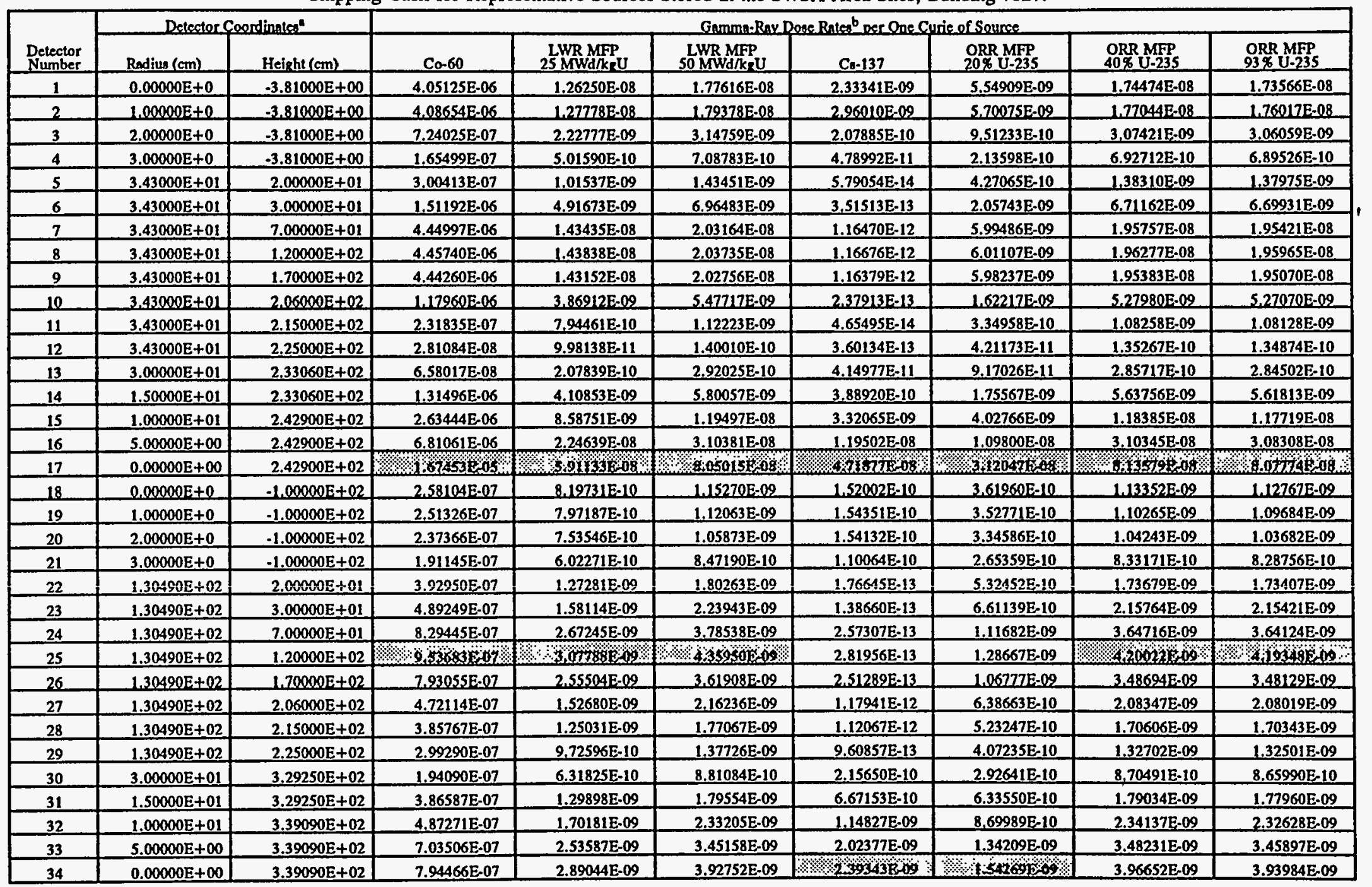

aDetector coordinates are relative to DORT zeometry model.
G Gamma-Ray dose units are (rem/hour)/(curie). 
Table 9. Gamma-Ray Dose Rates at Selected Detector Positions $3.81 \mathrm{~cm}$ and $1 \mathrm{~m}$ Away From the Surface of the In-Pile Loop LITR HB-2 Carrier Spent Nuclear Fuel Shipping Cask for Representative Sources Stored in the SWSA Area Sites, Building 7827.

\begin{tabular}{|c|c|c|c|c|c|c|c|c|c|}
\hline \multirow[b]{2}{*}{$\begin{array}{l}\text { Detector } \\
\text { Number } \\
\end{array}$} & \multicolumn{2}{|c|}{ Defecter Ceordinalea" } & \multicolumn{7}{|c|}{ Gamma-Rav Dope Ralea bec One Curie of Source } \\
\hline & $\operatorname{Radius}(\mathrm{cm})$ & Height (cm) & Co.60 & $\begin{array}{l}\text { LWR MFP } \\
25 \mathrm{MWd} / \mathrm{kg}\end{array}$ & $\begin{array}{c}L W R M F P \\
50 M W d / k R U\end{array}$ & Ca: 137 & $\begin{array}{l}\text { ORR MFP } \\
20 \% \mathrm{U}-235 \\
\end{array}$ & $\begin{array}{l}\text { ORR MFP } \\
40 \% \mathrm{U}-235 \\
\end{array}$ & $\begin{array}{l}\text { ORR MFP } \\
938 \mathrm{U}-235\end{array}$ \\
\hline 1 & $0.00000 E+0$ & $-3.81000 E+00$ & $173.359436,04$ & $\therefore \%$ i.22786女-07 & X & $1.80222 \mathrm{E}-08$ & $3,753 \times 0=02$ & \% & (in 1.2689406 \\
\hline 2 & $6.00000 \mathrm{E}+0$ & $-3.81000 E+\infty 0$ & $3.05943 \mathrm{E}-04$ & $8.42923 \mathrm{E}-07$ & $1.1977 \mathrm{UE} .06$ & $2,31747 E_{-} 08$ & $3.43982 E-07$ & $1.16477 \mathrm{E} .06$ & $1.15931 \mathrm{E}-06$ \\
\hline 3 & $1.20000 \mathrm{E}+0$ & $-3,81000 E+00$ & 1.99995E-04 & $5.65210 \mathrm{E}-07$ & $7.99338 \mathrm{E}-07$ & $28,70 \% 316.18$ & $2.38767 \mathrm{E}-07$ & $7.82523 \mathrm{E}-07$ & $7.78351 \mathrm{E}-07$ \\
\hline 4 & $1.80000 \mathrm{E}+0$ & $-3.81000 E+\infty$ & 4.78921E-0S & $1.35043 \mathrm{E}-07$ & $1.91643 \mathrm{E} .07$ & $6.14076 \mathrm{E}-09$ & $5.57000 \mathrm{E}-08$ & $1.86696 \mathrm{E}-07$ & $1.85809 \mathrm{E}-07$ \\
\hline 5 & $2.40000 E+0$ & $-3.81000 \mathrm{E}+00$ & $8.92007 \mathrm{E}-06$ & $2.56193 \mathrm{E}-08$ & $3.63427 \mathrm{E}-08$ & 1.12358E.09 & $1.06063 \mathrm{E}-08$ & 3.53775E-08 & 3,52204E-08 \\
\hline 6 & $3.06400 \mathrm{E}+01$ & $9.00000 E+\infty 0$ & $2.00006 \mathrm{E}-05$ & $5.67722 \mathrm{E}-08$ & $8.06595 \mathrm{E} .08$ & $2.58524 \mathrm{E}-10$ & $2.31582 \mathrm{E} .08$ & $7.81530 \mathrm{E}-08$ & $7,78536 \mathrm{E}-08$ \\
\hline 7 & $3.06400 \mathrm{E}+01$ & $1.80000 \mathrm{E}+01$ & $8.28306 \mathrm{E}-05$ & $2.30268 \mathrm{E}-07$ & $3.27336 \mathrm{E}-07$ & $1.53614 \mathrm{E}-09$ & $9.35404 \mathrm{E}-08$ & $3.17557 \mathrm{E}-07$ & $3.16228 \mathrm{E}-07$ \\
\hline 8 & $3.06400 E+01$ & $6,00000 \mathrm{E}+01$ & $1.67406 \mathrm{E}-04$ & $4.65977 \mathrm{E}-07$ & $6.62509 \mathrm{E}-07$ & $2.23585 \mathrm{E}-09$ & $1.89196 \mathrm{E}-07$ & $6,42310 \mathrm{E}-07$ & $6.39656 \mathrm{E}-07$ \\
\hline 9 & $3.06400 \mathrm{E}+01$ & $1.40000 E+02$ & $1.67345 \mathrm{E} .04$ & $4.65633 \mathrm{E}-07$ & $6.62020 \mathrm{E}-07$ & $2.23690 \mathrm{E}-09$ & $1.89091 \mathrm{E}-07$ & $6.41808 \mathrm{E}-07$ & 6.39169E-07 \\
\hline 10 & $3.06400 \mathrm{E}+01$ & $2.20000 E+02$ & $1.67437 \mathrm{E}=04$ & $4.66053 \mathrm{E}-07$ & $6.62622 \mathrm{E}-07$ & $2.23571 \mathrm{E}-09$ & $1.89228 \mathrm{E}=07$ & $6.42419 \mathrm{E}-07$ & $6.39771 \mathrm{E}-07$ \\
\hline 11 & $3.06400 E+01$ & $2.64000 \mathrm{E}+02$ & $8.26655 \mathrm{E}-05$ & $2.29811 \mathrm{E} .07$ & $3.26692 \mathrm{E}-07$ & $1.53341 \mathrm{E}-09$ & $9,33580 \mathrm{E}-08$ & $3.16932 \mathrm{E}-07$ & $3.15607 \mathrm{E}-07$ \\
\hline 12 & $3.06400 \mathrm{E}+01$ & $2.73000 \mathrm{E}+02$ & $1.99136 \mathrm{E}-05$ & $5.65284 \mathrm{E}-08$ & 8.03169E-08 & $2.57306 \mathrm{E}-10$ & 2.30593E-08 & $7.78185 \mathrm{E}-08$ & $7.75223 \mathrm{E}-08$ \\
\hline 13 & $2.40000 \mathrm{E}+01$ & $2.85790 \mathrm{E}+02$ & $8.92007 \mathrm{E} \cdot 06$ & 2.56194E.08 & $3.63428 \mathrm{E}-08$ & 1.12358E-09 & $1.06063 \mathrm{E}-08$ & $3,53776 \mathrm{E} \cdot 08$ & $3.52206 \mathrm{E} .08$ \\
\hline 14 & $1.80000 E+01$ & $2.85790 \mathrm{E}+02$ & 4.78919E-05 & $1.35043 \mathrm{E}-07$ & 1.91643E-07 & $6.14076 \mathrm{E}-09$ & $5,56998 \mathrm{E}-08$ & $1.86696 \mathrm{E}-07$ & $1.85809 \mathrm{E}-07$ \\
\hline 15 & $1.20000 \mathrm{E}+01$ & $2.85790 \mathrm{E}+02$ & $1.99995 \mathrm{E}-04$ & $5.65208 \mathrm{E}-07$ & $7.99336 \mathrm{E}-07$ & $6.79027 \mathrm{E}-08$ & $2.38766 \mathrm{E}-07$ & $7.82520 \mathrm{E} .07$ & $7.78348 \mathrm{E}-07$ \\
\hline 16 & $6.00000 E+00$ & $2.85790 \mathrm{E}+02$ & 3.05942E.04 & 8.42919E-07 & 1.19771E.06 & $2.31748 \mathrm{E}-08$ & $3.43980 \mathrm{E}-07$ & $1.16477 \mathrm{E}-06$ & 1.15930E-06 \\
\hline 17 & $0.00000 E+00$ & $2.85790 \mathrm{E}+02$ & 3.35942E-04 & $9.22782 \mathrm{E}-07$ & $1.31173 \mathrm{E}-06$ & $1.80222 \mathrm{E}-08$ & 3.75328E-07 & $1.27482 \mathrm{E}-06$ & 1.26894E-06 \\
\hline 18 & $0.00000 E+0$ & $-1.00000 E+02$ & $2.45388 \mathrm{E} \cdot 05$ & $6.86605 \mathrm{E}-08$ & $2.74375 E-08$ & $3.30409 \mathrm{E}-09$ & $2.83042 E-08$ & $2.49286 \mathrm{E}-08$ & $2.44759 \mathrm{E}-08$ \\
\hline 19 & $6.00000 E+0$ & $-1.00000 E+02$ & $2.42730 \mathrm{E}-05$ & $6,80454 \mathrm{E} \cdot 08$ & $9.65251 \mathrm{E}-08$ & $3.79076 \mathrm{E}-09$ & $2.81286 \mathrm{E}-08$ & 9.40691 E. 08 & 9.36190E. 08 \\
\hline 20 & $1.20000 \mathrm{E}+0$ & $-1.00000 \mathrm{E}+02$ & 2.33701E-05 & $6.57798 \mathrm{E}-08$ & $9.32286 \mathrm{E}-08$ & 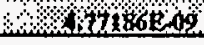 & 2.73586E-08 & $9.09315 \mathrm{E}-08$ & $9.04911 \mathrm{E}-08$ \\
\hline 21 & $1.80000 E+0$ & $-1.00000 E+02$ & $2,01119 \mathrm{E}=05$ & $5.66036 \mathrm{E}-08$ & $8.01934 \mathrm{E}-08$ & $4.57924 \mathrm{E}-09$ & $2.36034 \mathrm{E}-08$ & $7.82624 \mathrm{E}-08$ & 7.78769E.08 \\
\hline 22 & $2.40000 E+0$ & $-1.00000 E+02$ & 1.66834E-05 & $4.67541 \mathrm{E} .08$ & $6.62799 \mathrm{E} .08$ & $3.29406 \mathrm{E}-09$ & $1.94164 \mathrm{E}-08$ & $6.46663 \mathrm{E}-08$ & $6.43460 \mathrm{E}, 08$ \\
\hline 23 & $1.26830 \mathrm{E}+02$ & $9.00000 \mathrm{E}+\infty 0$ & $1.54296 \mathrm{E}-05$ & 4.30265E-08 & $6.11678 \mathrm{E}-08$ & $2.11464 \mathrm{E}-10$ & $1.74796 \mathrm{E}-08$ & $5.92952 \mathrm{E}-08$ & $5.90535 \mathrm{E}-08$ \\
\hline 24 & $1.26830 \mathrm{E}+02$ & $1.80000 E+01$ & $1.84773 \mathrm{E}-05$ & $5.14496 \mathrm{E} \cdot 08$ & $7.31455 \mathrm{E}-08$ & $2.65748 \mathrm{E}-10$ & $2.08965 \mathrm{E}=08$ & $7,09166 \mathrm{E} .08$ & $7.06248 \mathrm{E}-08$ \\
\hline 25 & $1.26830 \mathrm{E}+02$ & $6.00000 \mathrm{E}+01$ & 3.04513E-0S & $8.46083 \mathrm{E}-08$ & $1.20295 \mathrm{E}-07$ & 4.52412E-10 & $3.43492 \mathrm{E}-08$ & 1.16649E-07 & 1.16163E.07 \\
\hline 26 & $1.26830 \mathrm{E}+02$ & $1.40000 E+02$ & $8,606340.04$. & $80000200 \%$ & (1,476110.02t: & $5,00251 \mathrm{E}-10$ & $2 \times 10 \% s 600$ & W & 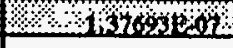 \\
\hline 27 & $1.26830 E+02$ & $2.20000 \mathrm{E}+02$ & 3.08322E-05 & $8.56704 \mathrm{E}-08$ & $1.21806 \mathrm{E}-07$ & $4.56900 \mathrm{E}-10$ & $3.47806 \mathrm{E}-08$ & $1,18112 E_{-07}$ & $1.17621 \mathrm{E}-07$ \\
\hline 28 & $1.26830 \mathrm{E}+02$ & $2.64000 \mathrm{E}+02$ & 1.84703E-05 & $5.14310 \mathrm{E}-08$ & $7.31192 \mathrm{E}, 08$ & $2.65632 \mathrm{E}-10$ & 2.08891E-08 & $7.08909 \mathrm{E} .08$ & 7.05994E $: 08$ \\
\hline 29 & $1.26830 \mathrm{E}+02$ & $2.73000 \mathrm{E}+02$ & $1.54228 \mathrm{E}-0 \mathrm{~S}$ & $4,30082 \mathrm{E}-08$ & $6.11419 \mathrm{E}-08$ & $2,11354 \mathrm{E}-10$ & $1.74724 \mathrm{E}-08$ & $5,92700 \mathrm{E}, 08$ & $5.90286 \mathrm{E}-08$ \\
\hline 30 & $2.40000 \mathrm{E}+01$ & $3.81980 \mathrm{E}+02$ & $1.66833 \mathrm{E}-05$ & 4.67539E. 08 & $6.62796 \mathrm{E}-08$ & 3.29404E 09 & 1,94163E-08 & $6,46660 \mathrm{E}-08$ & $6.43457 \mathrm{E}: 08$ \\
\hline 31 & $1.80000 E+01$ & $3.81980 E+02$ & $2.01118 \mathrm{E}-05$ & 5.66034E. 08 & $8.01930 \mathrm{E} \cdot 08$ & $4.57921 \mathrm{E}-09$ & 2.36032E-08 & $7.82620 \mathrm{E}_{-08}$ & 7.78765E .08 \\
\hline 32 & $1.20000 E+01$ & $3.81980 E+02$ & 2.33699 E.05 & $6.57795 \mathrm{E}-08$ & $9.32282 \mathrm{E}-08$ & $4.77183 \mathrm{E}-09$ & $2.73584 \mathrm{E}-08$ & $9,09310 \mathrm{E}-08$ & $9.04906 \mathrm{E}-08$ \\
\hline 33 & $6.00000 \mathrm{E}+00$ & $3.81980 E+02$ & 2.42729E-05 & $6.80450 \mathrm{E}-08$ & 9.65247E. 08 & 3.79074E-09 & 2.81284E-08 & $9.40686 \mathrm{E}, 08$ & $9.36186 \mathrm{E}-08$ \\
\hline 34 & $0.00000 \mathrm{E}+00$ & $3.81980 E+02$ & 2.45387E-05 & $6.86602 \mathrm{E}-08$ & $9.74371 \mathrm{E}=08$ & 3.30407E-09 & $2.83041 \mathrm{E}-08$ & $9.49281 \mathrm{E}-08$ & 9.44755E-08 \\
\hline
\end{tabular}

ADetector coordinatea are relative Lo DORT geometry model.
$b_{G}$ amman-Ray dose units are (rem/hour)/(curne). 
Table 10. Gamma-Ray Dose Rates at Selected Detector Positions $3.81 \mathrm{~cm}$ and $1 \mathrm{~m}$ Away From the Surface of the 6.5 Inch

HRLEL Carrier Spent Nuclear Fuel Shipping Cask for Representative Sources Stored in the SWSA Area Sites, Building 7827.

\begin{tabular}{|c|c|c|c|c|c|c|c|c|c|}
\hline \multirow[b]{2}{*}{$\begin{array}{l}\text { Delector } \\
\text { Number }\end{array}$} & \multicolumn{2}{|c|}{ Detector Coondinates" } & \multicolumn{7}{|c|}{ Gamma-Ray Dose Rates ber One Curie of Source } \\
\hline & Rodius (cm) & Height $(\mathrm{cm})$ & $\mathrm{C} 0.60$ & $\begin{array}{l}\text { LWR MFP } \\
25 \mathrm{MWd} / \mathrm{KB}_{8}\end{array}$ & $\begin{array}{l}\text { LWR MFP } \\
50 \mathrm{MWd} / \mathrm{kg} \mathrm{U}\end{array}$ & Cs.137 & $\begin{array}{l}\text { ORRMFP } \\
208 \mathrm{U}-235 \\
\end{array}$ & $\begin{array}{l}\text { ORR MPP } \\
408 \mathrm{U}-235 \\
\end{array}$ & $\begin{array}{l}\text { ORR MFP } \\
93 \% \text { U.235 } \\
\end{array}$ \\
\hline 1 & $0.00000 \mathrm{E}+00$ & $-3,81000 \mathrm{E}+00$ & $3,9420 \% 6,04$. & 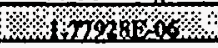 & $8,20850 \mathrm{n}, 06$ & Kagriseos & 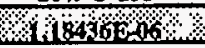 & 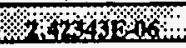 & 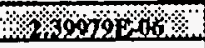 \\
\hline 2 & $3.00000 \mathrm{E}+00$ & $-3.81000 E+00$ & 3.57054 E. 04 & $1.60661 \mathrm{E} .06$ & $2.07618 \mathrm{E}-06$ & $2.79089 \mathrm{E}-06$ & $1.06734 \mathrm{E} .06$ & $2.18877 \mathrm{E} .06$ & $2.16742 \mathrm{E}-06$ \\
\hline 3 & $9.00000 \mathrm{E}+00$ & $-3.81000 E+\infty 0$ & $1.36824 \mathrm{E} .04$ & $5.88377 \mathrm{E}-07$ & $7.65898 \mathrm{E} \cdot 07$ & $9.50529 \mathrm{E}-07$ & $3.80308 \mathrm{E}-07$ & $8.04407 \mathrm{E}-07$ & $7.96543 \mathrm{E}-07$ \\
\hline 4 & $1.40000 \mathrm{E}+01$ & $-3.81000 \mathrm{E}+00$ & $5.23141 \mathrm{E} .05$ & 2.22784E-07 & $2.90388 \mathrm{E}-07$ & 3.54986E.07 & $1.43172 \mathrm{E}-07$ & $3.04626 \mathrm{E}-07$ & $3.01656 \mathrm{E}-07$ \\
\hline 5 & $2.50000 E+01$ & $-3.81000 E+\infty 0$ & 3.74095E.06 & 1.75391E-08 & $2.24757 \mathrm{E}-08$ & 3.31199E-08 & $1.20023 \mathrm{E}-08$ & 2.38253E. 08 & 2.35981 E-08 \\
\hline 6. & $3.30200 \mathrm{E}+01$ & $2,00000 E+01$ & 2.19434E.06 & $6.74266 \mathrm{E}-09$ & $2.53221 \mathrm{E}-09$ & $1.27761 \mathrm{E}-11$ & $2.78909 \mathrm{E}-09$ & $2.22275 \mathrm{E}-02$ & 2.12117E-09 \\
\hline 7 & $3.30200 \mathrm{E}+01$ & $3.50000 \mathrm{E}+01$ & $2.17129 \mathrm{E}-05$ & $6.41927 \mathrm{E}-08$ & $9.10850 \mathrm{E}-08$ & $1,39826 \mathrm{E}-10$ & $2.63899 \mathrm{E}-08$ & $8.80461 \mathrm{E}-08$ & $8.77758 \mathrm{E}-08$ \\
\hline 8 & $3.30200 \mathrm{E}+01$ & $7.50000 \mathrm{E}+01$ & 3.58875E-05 & $1.08078 \mathrm{E}-07$ & $1.53326 \mathrm{E}-07$ & $4.77746 \mathrm{E}-11$ & $4.45415 \mathrm{E}-08$ & $1.47953 \mathrm{E}-07$ & $1.47561 \mathrm{E}-07$ \\
\hline 9 & $3.30200 \mathrm{E}+01$ & $1.10000 E+02$ & $3.58362 \mathrm{E}-05$ & $1.07951 \mathrm{E}-07$ & $1.53154 \mathrm{E}-07$ & $4.77844 \mathrm{E}-11$ & 4.44994E-08 & $1.47823 \mathrm{E}-07$ & $1,47419 \mathrm{E}-07$ \\
\hline 10 & $3.30200 E+01$ & $1.30000 E+02$ & 3.16396E-05 & $9.48612 \mathrm{E}-08$ & $1.34587 \mathrm{E}-07$ & $4.54167 \mathrm{E}-11$ & $3.90879 \mathrm{E}-08$ & 1.29881E-07 & $1.29553 \mathrm{E} .07$ \\
\hline 11 & $3.87400 \mathrm{E}+01$ & $1.41000 E+02$ & 1.19541E.06 & 3.86404E-09 & 5.47361E-09 & $1.68612 \mathrm{E}-12$ & $1.61974 \mathrm{E}-09$ & $5.28366 \mathrm{E}=09$ & $5.27547 \mathrm{E}-09$ \\
\hline 12 & $3.87400 E+01$ & $1.62000 E+02$ & 3.79460E-08 & $1.30320 \mathrm{E}-10$ & $1.84907 \mathrm{E}-10$ & $2.42446 \mathrm{E}-14$ & $5.51208 \mathrm{E}-11$ & $1.78498 \mathrm{E}-10$ & $1.78110 \mathrm{E}-10$ \\
\hline 13 & $3.87400 E+01$ & $1.84000 E+02$ & $8.21457 \mathrm{E}-10$ & $2.69950 \mathrm{E}-12$ & $3.82326 \mathrm{E}-12$ & 3.50462E-15 & $1.13814 \mathrm{E}-12$ & $3.70367 \mathrm{E}-12$ & $3.69928 \mathrm{E}-12$ \\
\hline 14 & $3.87400 \mathrm{E}+01$ & $1.90000 E+02$ & $4.16525 \mathrm{E}-10$ & $1.35261 \mathrm{E}-12$ & $1.21582 \mathrm{E}-12$ & $2.66436 \mathrm{E}-15$ & $5.69403 \mathrm{E}-13$ & $1.85685 \mathrm{E}-12$ & $2.85447 E-12$ \\
\hline 15 & $2.00000 E+01$ & $2.02210 E+02$ & $1.74806 \mathrm{E}-08$ & $5.77096 \mathrm{E}-11$ & $8.17160 \mathrm{E}-11$ & $1.51809 \mathrm{E}-15$ & $2.42308 \mathrm{E}-11$ & $7.86831 \mathrm{E}-11$ & $7.85789 \mathrm{E}-11$ \\
\hline 16 & $1.00000 \mathrm{E}+01$ & $2.02210 \mathrm{E}+02$ & 5.98642E-07 & 2.01390E. 09 & 2.84999E.09 & $8.37134 \mathrm{E}-14$ & $8.48741 \mathrm{E}-10$ & 2.74514E-09 & $2.74159 \mathrm{E}-09$ \\
\hline 17 & $0.00000 \mathrm{E}+00$ & $2.02210 E+02$ & $1.31988 \mathrm{E} .06$ & 4.43271E.09 & $\begin{array}{r}6.27323 \mathrm{E}-09 \\
\end{array}$ & $2.80601 \mathrm{E}-13$ & $1.86728 \mathrm{E}-09$ & $6.04217 \mathrm{E} .09$ & $6.03447 \mathrm{E} .09$ \\
\hline 18 & $0.00000 E+00$ & $-1.00000 E+02$ & 1, & 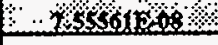 & 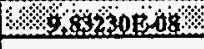 & 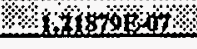 & 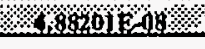 & 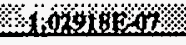 & 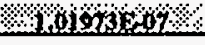 \\
\hline 19 & $3.00000 E+00$ & $-1.00000 E+02$ & $1.71236 \mathrm{E}-05$ & $7.46676 \mathrm{E}-08$ & $9.71472 \mathrm{E}-08$ & $1.20714 \mathrm{E}-07$ & 4.82849E-08 & $1.01707 \mathrm{E}_{0} 07$ & $1.00771 \mathrm{E} \cdot 07$ \\
\hline 20 & $9.00000 E+00$ & $-1.00000 \mathrm{E}+02$ & $1.46794 \mathrm{E}-0 \mathrm{~S}$ & $6.37276 \mathrm{E}=08$ & $8.29635 \mathrm{E}-08$ & 1.02430E:07 & $4.11218 \mathrm{E}-08$ & $8.68659 \mathrm{E}-08$ & $8.60609 \mathrm{E}-08$ \\
\hline 21 & $1.40000 \mathrm{E}+01$ & $-1.00000 \mathrm{E}+02$ & $1.18299 \mathrm{E}-05$ & 5.08085E-08 & $6.62557 \mathrm{E}-08$ & $8.02751 \mathrm{E}-08$ & 3.25809E-08 & $6.93452 \mathrm{E}_{-}-08$ & $6.86975 \mathrm{E} .08$ \\
\hline 22 & $2.50000 \mathrm{E}+01$ & $-400000 E+02$ & $7.61162 \mathrm{E}-06$ & 3. $39841 \mathrm{E}=08$ & $4.18477 E-08$ & $4.87821 \mathrm{E}=08$ & $2.02510 \mathrm{E}=08$ & $4,37613 \mathrm{E}=08$ & $4.33465 E-08$ \\
\hline 23 & $1.29210 \mathrm{E}+02$ & $2.00000 E+01$ & $3.01651 \mathrm{E}-06$ & $9.08507 \mathrm{E}-09$ & 1.28889E.08 & $9.08578 \mathrm{E}-12$ & $3.74607 \mathrm{E}-09$ & 1.24410E-08 & $1.24088 \mathrm{E} \cdot 08$ \\
\hline 24 & $1.29210 E+02$ & $3.50000 \mathrm{E}+01$ & 4.08397E-06 & $1.22671 \mathrm{E}-08$ & $1.74045 \mathrm{E}-08$ & $1.01546 \mathrm{E}-11$ & $5.05521 \mathrm{E}-09$ & $1.67988 \mathrm{E} .08$ & $1.67550 \mathrm{E}-08$ \\
\hline 25 & $1.29210 \mathrm{E}+02$ & $7.50000 \mathrm{E}+01$ & $6.17273 \mathrm{E}-06$ & $1.85049 \mathrm{E}-08$ & $2.62564 \mathrm{E} \cdot 08$ & $1.06369 \mathrm{E}-11$ & 7.62259E-09 & $2.53389 \mathrm{E}-08$ & $2.52729 \mathrm{E}-08$ \\
\hline 26 & $1.29210 E+02$ & $1.10000 \mathrm{E}+02$ & $5.63233 \mathrm{E}-06$ & $1.69147 \mathrm{E}-08$ & $2.39993 \mathrm{E}-08$ & $9.41508 \mathrm{E}-12$ & $6.97024 \mathrm{E}-09$ & $2.31591 \mathrm{E}-08$ & $2.30994 \mathrm{E}-08$ \\
\hline 27 & $1.29210 \mathrm{E}+02$ & $1.30000 \mathrm{E}+02$ & 4.14068E-06 & $1.24766 \mathrm{E}-08$ & $1.77010 \mathrm{E}-08$ & $6.19321 \mathrm{E}-12$ & $5.14478 \mathrm{E}-09$ & $1.70798 \mathrm{E}-08$ & 1.70365E-08 \\
\hline 28 & $1.34930 \mathrm{E}+02$ & $1.41000 E+02$ & 3.04849E-06 & $9.21628 \mathrm{E}-09$ & 1.30744E-08 & $3.97217 \mathrm{E}-12$ & $3.80286 \mathrm{E}-09$ & $1.26145 \mathrm{E}-08$ & $1.25831 \mathrm{E}-08$ \\
\hline 29 & $1.34930 E+02$ & $1.62000 \mathrm{E}+02$ & $1.61935 \mathrm{E}-06$ & 4.93689E-09 & $7.00210 \mathrm{E}-09$ & 1.37614E-12 & 2.04035E.09 & $6.75456 \mathrm{E}-09$ & $6.73848 \mathrm{E}-09$ \\
\hline 30 & $134930 E+02$ & $1.84000 \mathrm{E}+02$ & $7.51365 E-07$ & $2.30130 E-09$ & $3.26357 \mathrm{E}-09$ & $4.51042 \mathrm{E}-13$ & $2.51872 \mathrm{E}-10$ & 3.14810E-09 & 3.14078E-09 \\
\hline 31 & $1.34930 \mathrm{E}+02$ & $1.90000 \mathrm{E}+02$ & $6.10532 \mathrm{E} .07$ & $1.86945 \mathrm{E}-09$ & $2.65114 \mathrm{E}-09$ & $3.57450 \mathrm{E}-13$ & $7.73182 E_{-10}$ & $2.55741 \mathrm{E}, 09$ & $2.55146 \mathrm{E}-09$ \\
\hline 32 & $2.00000 \mathrm{E}+01$ & $2.98400 \mathrm{E}+02$ & $6.27837 \mathrm{E}-08$ & $2.15400 \mathrm{E}-10$ & $3.04701 \mathrm{E}-10$ & 1.73934E- -14 & $9.11097 \mathrm{E}-11$ & $2.93505 \mathrm{E}-10$ & $2.93173 \mathrm{E}-10$ \\
\hline 33 & $1.00000 E+01$ & $2.98400 E+02$ & 1.03670E.07 & $3.63315 E-10$ & $5.13721 \mathrm{E}-10$ & $4,21982 E-14$ & $1.54241 \mathrm{E}-10$ & 4. $94794 \mathrm{E}-10$ & $4.94346 \mathrm{E}-10$ \\
\hline 34 & & $2.98400 E+02$ & $1.09517 \mathrm{E}-07$ & $3.83497 \mathrm{E}-10$ & S.42267E-10 & 4.56218E-14 & $1.62786 \mathrm{E}-10$ & $5.22293 \mathrm{E}-10$ & $5.21815 \mathrm{E}-10$ \\
\hline
\end{tabular}

2Detector coond inates are relative to DORT geometry model.
bamma-Ray dose cunits are (rem/hour)/(cure). 
Table 11. Gamma-Ray Dose Rates at Selected Detector Positions $3.81 \mathrm{~cm}$ and $1 \mathrm{~m}$ Away From the Surface of the HFIR

Hot Scrap and Spent Nuclear Fuel Shipping Cask for Representative Sources Stored in the SWSA Area Sites, Building 7827.

\begin{tabular}{|c|c|c|c|c|c|c|c|c|c|}
\hline \multirow[b]{2}{*}{$\begin{array}{l}\text { Detector } \\
\text { Number }\end{array}$} & \multicolumn{2}{|c|}{ Detector Coondinates: } & \multicolumn{7}{|c|}{ Gemmi-Ray Dose Rales ber One Curie of Source } \\
\hline & Radius (cm) & Meipht $(\mathrm{cm})$ & ${ }^{\infty} \mathrm{Co}$ & $\begin{array}{l}\text { LWR MFP } \\
25 \mathrm{MWd} / \mathrm{kg} \mathrm{U}\end{array}$ & $\begin{array}{l}\text { LWR MFP } \\
\text { 50 MWd ks }\end{array}$ & ${ }^{137} \mathrm{Cs}$ & $\begin{array}{l}\text { ORR MFP } \\
20 \% \text { US }\end{array}$ & $\begin{array}{l}\text { ORR MFP } \\
40 \% \text { US }\end{array}$ & $\begin{array}{l}\text { ORR MFP } \\
938 \\
\end{array}$ \\
\hline 1 & $0.00000 \mathrm{E}+00$ & $-3.81000 \mathrm{E}+00$ & 2.33952E-06 & $7.69303 \mathrm{E}-09$ & $1.07656 \mathrm{E} \cdot 08$ & $2.03302 \mathrm{E}-09$ & 3.49447E-09 & $1.06010 \mathrm{E}-08$ & $1.05508 \mathrm{E}-08$ \\
\hline 2 & $9,00000 \mathrm{E}+00$ & $-2,86000 \mathrm{E}+00$ & $6.98577 \mathrm{E}-07$ & $2,40801 \mathrm{E} .09$ & $3.39480 \mathrm{E} .09$ & $1,76782 \mathrm{E}-10$ & $1.04283 \mathrm{E}-09$ & $3.29270 \mathrm{E}-09$ & $3,28654 \mathrm{E}-09$ \\
\hline 3 & $1.80000 \mathrm{E}+01$ & $-2.86000 \mathrm{E}+00$ & $8.07562 \mathrm{E}-07$ & $2.61450 \mathrm{E} .09$ & 3.69893E.09 & $8.16835 \mathrm{E}-11$ & $1,10533 \mathrm{E}-09$ & $3.58410 \mathrm{E}-09$ & $3.57478 \mathrm{E}-09$ \\
\hline 4 & $2.80000 \mathrm{E}+01$ & $-2.86000 \mathrm{E}+00$ & 4.87254E-07 & $1.61992 \mathrm{E}-09$ & $2.29277 \mathrm{E} \cdot 09$ & $6.56894 \mathrm{E}-12$ & $6.82245 \mathrm{E}-10$ & 2.21238E.09 & $2.20838 \mathrm{E}-09$ \\
\hline 5 & $3.80000 \mathrm{E}+01$ & $-2.86000 \mathrm{E}+00$ & $1,79078 \mathrm{E} \cdot 07$ & $5.88129 \mathrm{E}-10$ & $8.32590 \mathrm{E}-10$ & $2.10165 \mathrm{E}-12$ & $2.47074 \mathrm{E}-10$ & $8.03122 \mathrm{E}-10$ & $8.01637 \mathrm{E}-10$ \\
\hline 6 & $4.38200 E+01$ & $1.60000 E+01$ & $1.61950 \mathrm{E}_{-07}$ & $5.71091 \mathrm{E}-10$ & $8.07689 \mathrm{E}-10$ & $1.09546 \mathrm{E}-13$ & $2.42850 \mathrm{E}-10$ & $7.78036 \mathrm{E}-10$ & $7.77531 \mathrm{E}-10$ \\
\hline 7 & $4.38200 E+01$ & 3.10000E+01 & $1.83374 \mathrm{E}-06$ & $6.04930 \mathrm{E}-09$ & $8.56576 \mathrm{E}-09$ & $6.46230 \mathrm{E}-13$ & $2.54084 \mathrm{E}-09$ & $8.25169 \mathrm{E}-09$ & $8.23777 \mathrm{E}-09$ \\
\hline 8 & $4.38200 E+01$ & $5.40000 \mathrm{E}+01$ & 3.60398E.06 & $1.18520 \mathrm{E} \cdot 08$ & $1.67787 \mathrm{E}-08$ & $2.48379 \mathrm{E}-12$ & $4.97231 \mathrm{E}-09$ & $1.61680 \mathrm{E}-08$ & 1.61438E-08 \\
\hline 9 & $4.38200 \mathrm{E}+01$ & $7.70000 \mathrm{E}+01$ & 3.71443E-06 & $1.22531 \mathrm{E}-08$ & $1.73467 \mathrm{E} \cdot 08$ & $1.76057 \mathrm{E}-11$ & $5.14508 \mathrm{E}-09$ & 1.67125E-08 & $1.66893 \mathrm{E}-08$ \\
\hline 10 & $4.38200 E+01$ & $1.00000 \mathrm{E}+02$ & $3,68462 \mathrm{E}=06$ & $121529 \mathrm{E}-08$ & $1.71817 \mathrm{E} .08$ & $3.23702 \mathrm{E}-10$ & $5.14196 \mathrm{E}-09$ & 165809E-08 & 165546E-08 \\
\hline 11 & $4.38200 \mathrm{E}+01$ & $1.23000 \mathrm{E}+02$ & Sifit3ation & 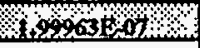 & 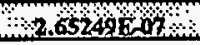 & 25T7021 01 & W. 1962260 & $2305 x+4+607$ & $2,726040.02$ \\
\hline 12 & $4.38200 E+01$ & $1.34000 \mathrm{E}+02$ & $2.36028 \mathrm{E} \cdot 06$ & $7.98814 \mathrm{E}-09$ & $1.10196 \mathrm{E}-08$ & $4.25416 \mathrm{E}-09$ & $3.91010 \mathrm{E}-09$ & $1.09663 \mathrm{E}-08$ & $1.09105 \mathrm{E}-08$ \\
\hline 13 & $4.38200 \mathrm{E}+01$ & $1,45000 \mathrm{E}+02$ & $1.91411 \mathrm{E} \cdot 07$ & $6.79733 \mathrm{E}-10$ & $9,31020 \mathrm{E}-10$ & $4,30450 \mathrm{E}-10$ & $3.43816 \mathrm{E}-10$ & $9.32018 \mathrm{E}_{-10}$ & $9.26614 \mathrm{E}-10$ \\
\hline 14 & $3.20000 \mathrm{E}+01$ & $1.52720 \mathrm{E}+02$ & $2,89108 \mathrm{E}-07$ & $9,78186 \mathrm{E}-10$ & $1.38369 \mathrm{E}-09$ & $2.33247 \mathrm{E}-12$ & $4.13064 \mathrm{E}-10$ & $1,33403 \mathrm{E}-09$ & $1.33228 \mathrm{E}-09$ \\
\hline 15 & $2.12500 \mathrm{E}+01$ & $1.52720 \mathrm{E}+02$ & $4.19370 \mathrm{E}-06$ & $1.30722 \mathrm{E}-08$ & $1.85088 \mathrm{E} .08$ & $4.26577 \mathrm{E}-10$ & 5.48615E-09 & $1.79340 \mathrm{E}-08$ & $1.78828 \mathrm{E}-08$ \\
\hline 16 & $1.10000 \mathrm{E}+01$ & $1.51450 \mathrm{E}+02$ & $6.41341 \mathrm{E}-06$ & $2.07393 \mathrm{E}_{0} 08$ & $2.93700 \mathrm{E}-08$ & $1.41414 \mathrm{E}-11$ & 8.67577E-09 & $2.82958 \mathrm{E} \cdot 08$ & $2.82509 \mathrm{E}-08$ \\
\hline 17 & $0.00000 E+00$ & $1.51450 \mathrm{E}+02$ & $8.21031 \mathrm{E} .06$ & $2.63923 \mathrm{E}-08$ & $3.73823 \mathrm{E}-08$ & $8.19659 \mathrm{E}-12$ & $1.10282 \mathrm{E}-08$ & 3.60223E-08 & 3.59557E-08 \\
\hline 18 & $0.00000 \mathrm{E}+00$ & $-1,00000 \mathrm{E}+02$ & $1.25505 \mathrm{E}-07$ & $4.24985 E-10$ & $5.98004 \mathrm{E}-10$ & S.38811E- 11 & $1.86421 \mathrm{E}-10$ & $5.82272 \mathrm{E}-10$ & $5.80594 \mathrm{E}-10$ \\
\hline 19 & $9.00000 \mathrm{E}+00$ & $-9.90500 \mathrm{E}+01$ & 1.25687E-07 & $4.23895 \mathrm{E}-10$ & $5.96882 \mathrm{E}-10$ & $4.82933 \mathrm{E}-11$ & $1.85113 \mathrm{E}-10$ & $5.80828 \mathrm{E}-10$ & $5.79178 \mathrm{E}-10$ \\
\hline 20 & $1.80000 \mathrm{E}+01$ & $-9.90500 \mathrm{E}+01$ & $1.15348 \mathrm{E}-07$ & $3.84228 \mathrm{E}, 10$ & $5.42563 \mathrm{E}-10$ & $2.26713 \mathrm{E}-11$ & $1.64715 \mathrm{E}-10$ & $5.26532 \mathrm{E}-10$ & $5.25165 \mathrm{E}-10$ \\
\hline 21 & $2.80000 \mathrm{E}+01$ & $-9.90500 \mathrm{E}+01$ & 9.95551E.08 & $3.31416 \mathrm{E}-10$ & $4.68295 \mathrm{E}-10$ & $1.45757 \mathrm{E}-11$ & $1,41412 \mathrm{E}-10$ & $4.53872 \mathrm{E}-10$ & $4.52776 \mathrm{E}-10$ \\
\hline 22 & $3.80000 \mathrm{E}+01$ & $-9.90500 \mathrm{E}+01$ & $8.55284 \mathrm{E}-08$ & $2.86716 \mathrm{E}-10$ & $4.05234 \mathrm{E} \cdot 10$ & $9.80159 \mathrm{E}-12$ & $1.22130 \mathrm{E}-10$ & $3,92308 \mathrm{E}-10$ & 3.91445E: 10 \\
\hline 23 & $1.40010 \mathrm{E}+02$ & $1.60000 \mathrm{E}+01$ & $3.83127 \mathrm{E}-07$ & $1.30209 \mathrm{E}-09$ & $1.82463 \mathrm{E}-09$ & $2.69153 \mathrm{E} \cdot 10$ & $5.84264 \mathrm{E}-10$ & $1.77839 \mathrm{E}-09$ & $1.77363 \mathrm{E} \cdot 09$ \\
\hline 24 & $1.40010 \mathrm{E}+02$ & $3.10000 \mathrm{E}+01$ & 5.33494E-07 & $1.80540 \mathrm{E}-09$ & $2.52946 \mathrm{E} \cdot 09$ & $3.83503 \mathrm{E}-10$ & $8.10890 \mathrm{E}-10$ & $2.46641 E-09$ & $2.45961 \mathrm{E}-09$ \\
\hline 25 & $1.40010 \mathrm{E}+02$ & $5.40000 \mathrm{E}+01$ & $7.70823 \mathrm{E}-07$ & 2.60614E-09 & $3.64321 \mathrm{E}-09$ & $6.80038 \mathrm{E}-10$ & $1.18679 \mathrm{E}_{0} 09$ & $3.56354 \mathrm{E}-09$ & $3.55222 \mathrm{E}-09$ \\
\hline 26 & $1.40010 \mathrm{E}+02$ & $770000 \mathrm{E}+01$ & $2.91316 \mathrm{E}-07$ & $3.37773 \mathrm{E}_{c} 02$ & $4.69556 \mathrm{E}-09$ & $1,27434 \mathrm{E}-09$ & $1.59109 \mathrm{E}_{2} 02$ & $4.62698 \mathrm{E}-09$ & $4.60810 \mathrm{E}-09$ \\
\hline 27 & $1.40010 \mathrm{E}+02$ & $1,00000 \mathrm{E}+02$ & $1,25791 \mathrm{E}-06$ & 4.37810 -09 & 6.02107E.09 & $2.61293 \mathrm{E}-09$ & $2.19377 \mathrm{E}-09$ & $6.01487 \mathrm{E} .09$ & $5.98078 \mathrm{E}-09$ \\
\hline 28 & $1.40010 \mathrm{E}+02$ & $1.23000 \mathrm{E}+02$ & $1116600 \%$ & 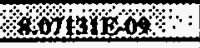 & \%1:081740 E08 & ariosme os & 160486009 & OLTHZEOB & 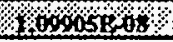 \\
\hline 29 & $1.40010 E+02$ & $1.34000 E+02$ & $1.80253 \mathrm{E}-06$ & $6.56254 \mathrm{E}-09$ & 8.87867E-09 & $6.02358 \mathrm{E}-09$ & $3,58052 \mathrm{E}, 09$ & $9.02712 \mathrm{E}-09$ & $8.95965 \mathrm{E}-09$ \\
\hline 30 & $1.40010 \mathrm{E}+02$ & $1,45000 \mathrm{E}+02$ & $1.12676 \mathrm{E}-06$ & $3.89465 \mathrm{E}-09$ & 5.33585E-09 & $2.65477 \mathrm{E}-09$ & $1.99259 \mathrm{E} .09$ & $5,36384 \mathrm{E}, 09$ & $5,32851 \mathrm{E}-09$ \\
\hline 31 & $3.20000 \mathrm{E}+01$ & $2.48910 \mathrm{E}+02$ & 6.13923E-07 & $1.97430 \mathrm{E} \cdot 09$ & 2.79576E-09 & 1.84279E-11 & $8.27384 \mathrm{E}-10$ & 2.69826E-09 & 2.69308E-09 \\
\hline 32 & $2.12500 \mathrm{E}+01$ & $2.48910 \mathrm{E}+02$ & $7.59203 \mathrm{E}-07$ & $2.43609 \mathrm{E} .09$ & 3.44945E-09 & $2.98062 \mathrm{E}-11$ & $1.02136 \mathrm{E}, 09$ & $3,33009 \mathrm{E}-09$ & $3,32353 \mathrm{E}-09$ \\
\hline 33 & $1.10000 \mathrm{E}+01$ & $2.47640 \mathrm{E}+02$ & 8.52423E-07 & 2.73523E-09 & $3.87390 \mathrm{E}-09$ & $1.81814 \mathrm{E}-11$ & $1.14478 \mathrm{E}-09$ & $3.73760 \mathrm{E}-09$ & $3.73058 E_{0} 09$ \\
\hline 34 & $0.00000 \mathrm{E}+00$ & $2.47640 \mathrm{E}+02$ & $8.81912 \mathrm{E}-07$ & 2.82948E-09 & 4.00765E-09 & $1.40253 \mathrm{E}-11$ & $1.18356 \mathrm{E}-09$ & $3.86582 \mathrm{E}, 09$ & $3.85868 \mathrm{E}-09$ \\
\hline
\end{tabular}

Detector coordinates are relative to DORT geometry model.

bGamma-Ray dose units are (rem/hour)/(curie). 
Table 12. Gamma-Ray Dose Rates at Selected Detector Positions $3.81 \mathrm{~cm}$ and $1 \mathrm{~m}$ Away From the Surface of the 10 Inch ORR Experiment Removal Shield Spent Nuclear Fuel Shipping Cask for Representative Sources Stored in the SWSA Area Sites, Building 7827.

\begin{tabular}{|c|c|c|c|c|c|c|c|c|c|}
\hline \multirow[b]{2}{*}{$\begin{array}{l}\text { Detector } \\
\text { Number }\end{array}$} & \multicolumn{2}{|c|}{ Detector Coondinates" } & \multicolumn{7}{|c|}{ Gamma-Ray Dose Rates ber One Curie of Sounce } \\
\hline & Radius $(\mathrm{cm})$ & Height $(\mathrm{cm})$ & $C_{0}-60$ & $\begin{array}{l}\text { LWR MFP } \\
25 \mathrm{MWd} / \mathrm{kg} \mathrm{U}\end{array}$ & $\begin{array}{c}\text { LWR MFP } \\
50 \mathrm{MWd} / \mathrm{kg} \mathrm{U}\end{array}$ & Ca.137 & $\begin{array}{l}\text { ORR MFP } \\
20 \% \text { U.235 }\end{array}$ & $\begin{array}{l}\text { ORR MFP } \\
40 \% \text { U-235 }\end{array}$ & $\begin{array}{l}\text { ORR MFP } \\
93 \% \text { U.235 } \\
\end{array}$ \\
\hline 1 & $0.00000 \mathrm{E}+00$ & $0.00000 E+00$ & $4.99486 \mathrm{E}-06$ & $1.54614 \mathrm{E}-08$ & $2.18905 \mathrm{E} \cdot 08$ & $4.85992 \mathrm{E}-10$ & $6.47467 \mathrm{E}-09$ & 2.11671E-08 & $2.11192 \mathrm{E}-0 \mathrm{~B}$ \\
\hline 2 & $2,00000 E+00$ & $0.00000 \mathrm{E}+00$ & $4.52248 \mathrm{E}-06$ & $1.40544 \mathrm{E}-08$ & $1.98742 \mathrm{E} .08$ & $8.37599 \mathrm{E}-10$ & S.94115E-09 & $1.92564 \mathrm{E} \cdot 08$ & $1.92038 \mathrm{E} \cdot 08$ \\
\hline 3 & $1.80000 \mathrm{E}+0$ & $.3 .81000 \mathrm{E}+00$ & $2.05460 \mathrm{E} \cdot 06$ & $6.27199 \mathrm{E}-09$ & $8.83646 \mathrm{E}-09$ & $1.01609 \mathrm{E} \cdot 09$ & $2.72748 \mathrm{E}-09$ & 8.66584E-09 & 8.62479E-09 \\
\hline 4 & $2.60000 \mathrm{E}+0$ & $-3.81000 \mathrm{E}+00$ & $6.80733 \mathrm{E}-07$ & $2.07576 \mathrm{E}-09$ & $2.93350 \mathrm{E}-09$ & $1.98421 \mathrm{E}-10$ & $8.84744 \mathrm{E}-10$ & 2.86493E-09 & $2.85261 \mathrm{E}-09$ \\
\hline 5 & $4.06400 \mathrm{E}+01$ & $2.70000 \mathrm{E}+01$ & $1.93143 \mathrm{E}-06$ & 5.78705E-09 & $8.20800 \mathrm{E} \cdot 09$ & $6.97602 \mathrm{E}-11$ & 2.39462E-09 & 7.93735E-09 & $7.91432 \mathrm{E}-09$ \\
\hline 6 & $4.06400 \mathrm{E}+01$ & $8.90000 E+01$ & 2.25013E-06 & $7.07156 \mathrm{E}-09$ & $1.00135 \mathrm{E}-08$ & $1.32860 \mathrm{E}-10$ & 2.95773E-09 & 9.66301E-09 & $9.64231 \mathrm{E}-09$ \\
\hline 7 & $4.06400 \mathrm{E}+01$ & $1.51000 \mathrm{E}+02$ & 2.30304E-06 & $7.30361 \mathrm{E}-09$ & $1.03116 \mathrm{E}-08$ & $5.18148 \mathrm{E}-10$ & 3.10925E-09 & 9.98005E-09 & 9.95490E.09 \\
\hline 8 & $4.38200 \mathrm{E}+01$ & $2.13000 \mathrm{E}+02$ & $2.39431 \mathrm{E} \cdot 05$ & $8.43446 \mathrm{E}-08$ & 1.14168E-07 & $7.82158 \mathrm{E}-08$ & $4.59255 \mathrm{E}-08$ & $1.16360 \mathrm{E}-07$ & $1.15420 \mathrm{E}-07$ \\
\hline 9 & $3.55600 \mathrm{E}+01$ & $2.73000 \mathrm{E}+02$ & $5.98499 \mathrm{E}-05$ & $1.74223 \mathrm{E}-07$ & $2.45245 \mathrm{E}-07$ & $3.31648 \mathrm{E}-08$ & $7.55966 \mathrm{E}-08$ & 2.39565E-07 & 2.38483E-07 \\
\hline 10 & $4.38200 \mathrm{E}+01$ & $3,33000 \mathrm{E}+02$ & $3.66634 \mathrm{E}=05$ & $121883 \mathrm{E}=07$ & $1,66832 \mathrm{E} \cdot 07$ & 8.780200008 & $6.25867 E_{-0} 08$ & $1.68038 \mathrm{E}_{-}-07$ & $1.66838 \mathrm{E} \cdot 07$ \\
\hline 11 & $3.55600 \mathrm{E}+01$ & $3.93000 \mathrm{E}+02$ & $5.95397 \mathrm{E} .05$ & $1.72436 \mathrm{E} \cdot 07$ & 2.43045E-07 & $2.86662 \mathrm{E}-08$ & $7.42139 \mathrm{E}-08$ & $2.37178 \mathrm{E}-07$ & 2.36134E-07 \\
\hline 12 & $4.38200 \mathrm{E}+01$ & $4.53000 \mathrm{E}+02$ & $2.45343 \mathrm{E}-05$ & 8.25796E-08 & $1.12717 \mathrm{E} \cdot 07$ & $6.37515 \mathrm{E}-08$ & $4.30294 \mathrm{E}-08$ & 1.13805E-07 & $1.12975 \mathrm{E} \cdot 07$ \\
\hline 13 & $3.55600 \mathrm{E}+01$ & $4.65000 E+02$ & $5.15851 \mathrm{E} \cdot 05$ & $2.25169 \mathrm{E}-07$ & $2.91543 \mathrm{E}-07$ & $3.84092 \mathrm{E}-07$ & $1,48068 \mathrm{E}-07$ & $3,05029 \mathrm{E}-07$ & $3.02356 \mathrm{E} \cdot 07$ \\
\hline 14 & $2.40000 E+01$ & $4.81360 \mathrm{E}+02$ & 1.76315E.05 & $5.30378 \mathrm{E}-08$ & $7,41611 \mathrm{E}-08$ & $1.79702 \mathrm{E}-08$ & $2.42143 \mathrm{E}-08$ & $7,37133 \mathrm{E}-08$ & $7.32146 \mathrm{E} \cdot 08$ \\
\hline 15 & $1.80000 \mathrm{E}+01$ & $4.81360 \mathrm{E}+02$ & $3.45060 \mathrm{E}-05$ & 1.04734E-07 & $1.46003 \mathrm{E} \cdot 07$ & 4.11035E-08 & $4.85880 \mathrm{E}-08$ & $1.44999 \mathrm{E}-07$ & 1.44072E-07 \\
\hline 16 & $9.00000 \mathrm{E}+00$ & $4.78180 \mathrm{E}+02$ & $9.92879 \mathrm{E}-05$ & $2.80159 \mathrm{E}-07$ & $3.96742 \mathrm{E}-07$ & $2.40959 \mathrm{E} \cdot 08$ & $1.17022 \mathrm{E}-07$ & $3.86510 \mathrm{E}-07$ & $3.84726 \mathrm{E}-07$ \\
\hline 17 & $0.00000 \mathrm{E}+00$ & $4.78180 E+02$ & 1713646404 & 31744 aton? & $\triangle O S 050 R \mathrm{E} O 23$ & 1.47984E-08 & 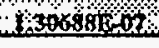 & 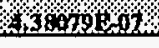 & 43365stur \\
\hline 18 & $0.00000 E+0$ & $-2,61900 \mathrm{E}+01$ & $4.96810 \mathrm{E}-07$ & $1.55793 \mathrm{E} .09$ & $2.20357 \mathrm{E} .09$ & $8.11260 \mathrm{E}-11$ & $6.58447 \mathrm{E}-10$ & $2.13678 \mathrm{E}-09$ & $2.13067 \mathrm{E}=09$ \\
\hline 19 & $9.00000 \mathrm{E}+0$ & $-9.61900 \mathrm{E}+01$ & $4.85112 \mathrm{E}-07$ & $1.52198 \mathrm{E}-09$ & 2.15255E.09 & 8.17796E-11 & $6.43646 \mathrm{E}-10$ & 2.08767E.09 & 2.08165E.09 \\
\hline 20 & $1.80000 \mathrm{E}+0$ & $-1.00000 \mathrm{E}+02$ & $4.18406 \mathrm{E}-07$ & $1.31194 \mathrm{E}-09$ & $1.85504 \mathrm{E}-09$ & $7.85526 \mathrm{E}-11$ & $5.55821 \mathrm{E}-10$ & $1.80023 \mathrm{E} .09$ & $1.79487 \mathrm{E}-09$ \\
\hline 21 & $2.60000 \mathrm{E}+0$ & $-1.00000 \mathrm{E}+02$ & $3.44729 \mathrm{E}-07$ & $1.07260 \mathrm{E}-09$ & $1.51626 \mathrm{E}-09$ & $7.50869 \mathrm{E}-11$ & $4.55193 \mathrm{E}-10$ & 1.47317E.09 & 1,46840F.09 \\
\hline $2 \hat{2}$ & i. $36830 \mathrm{E}+02$ & $2.70000 \mathrm{E}+01$ & $5.99346 \mathrm{E}-07$ & $2.37052 \mathrm{E}-0 \%$ & $3.15725 E-09$ & $2.79235 \mathrm{E}-09$ & 1.38395E-09 & $3.21055 E .09$ & $3.190955-09$ \\
\hline 23 & $1.36830 \mathrm{E}+02$ & $8.90000 \mathrm{E}+01$ & $1.10183 \mathrm{E} \cdot 06$ & 4.25397E-09 & $5.68978 \mathrm{E}-09$ & $4.69922 \mathrm{E}-09$ & $2.43683 \mathrm{E}-09$ & $5.76954 \mathrm{E}-09$ & 5.73472E-09 \\
\hline 24 & $1.36830 \mathrm{E}+02$ & $1.51000 \mathrm{E}+02$ & 2.3520SE.06 & $8.73662 \mathrm{E}-09$ & $1.17250 \mathrm{E}-08$ & $9.23179 \mathrm{E} \cdot 09$ & $4.92898 \mathrm{E} .09$ & 1.18877E.08 & $1.18110 \mathrm{E}-08$ \\
\hline 25 & $1.40010 \mathrm{E}+02$ & $2.13000 \mathrm{E}+02$ & $8.80853 \mathrm{E} .06$ & 2.99414E-08 & 4.07491E-08 & $2.44516 \mathrm{E}-08$ & $1.57800 \mathrm{E}-08$ & 4.09541E-08 & $4.06942 \mathrm{E}-08$ \\
\hline 26 & $1.31750 \mathrm{E}+02$ & $2.73000 E+02$ & $1.94467 \mathrm{E}-05$ & $211599 E_{-08}$ & $9.53018 E_{-} 08$ & $7.88115 \mathrm{E}-08$ & $4.05498 \mathrm{E} \cdot 08$ & $9.70137 \mathrm{E} .08$ & $9.63229 \mathrm{E}-08$ \\
\hline 27 & $1.40010 \mathrm{E}+02$ & $3.33000 \mathrm{E}+02$ & 1.54312E-05 & 5.11899E-08 & $6.99762 \mathrm{E}-08$ & $3,77500 \mathrm{E}-08$ & $2.63656 \mathrm{E}-08$ & $7,00750 \mathrm{E}-08$ & $6.96448 \mathrm{E}-08$ \\
\hline 28 & $1.31750 E+02$ & $3.93000 \mathrm{E}+02$ & 1.76180E-0S & 6.39782E-08 & $8.58058 \mathrm{E}-08$ & $6.91283 \mathrm{E}-08$ & $3.62085 \mathrm{E}-08$ & $8.71872 \mathrm{E}-08$ & $8.65823 \mathrm{E}-08$ \\
\hline 29 & $1.40010 \mathrm{E}+02$ & $4.53000 \mathrm{E}+02$ & $9.02993 \mathrm{E}-06$ & $3.10276 \mathrm{E}-08$ & 4.20909E-08 & 2.72214E-08 & $1.66210 \mathrm{E}-08$ & $4.24239 \mathrm{E}-08$ & $4.21453 \mathrm{E}=08$ \\
\hline 30 & $1.31750 \mathrm{E}+02$ & $4.65000 E+02$ & 9.43877E-06 & $3.56568 \mathrm{E}-08$ & $4.74621 \mathrm{E}-08$ & 4,34044E-08 & $2.08945 \mathrm{E}-08$ & $4.85874 \mathrm{E}-08$ & $4.82221 \mathrm{E}-08$ \\
\hline 31 & $2.40000 \mathrm{E}+01$ & $5.77550 \mathrm{E}+02$ & 7.22467E-06 & $2.09135 \mathrm{E}-08$ & 2.94967E-08 & 3.35878E-09 & $8.98891 \mathrm{E}-09$ & 2.88785E-08 & 2.87336E-08 \\
\hline 32 & $1.80000 \mathrm{E}+01$ & $5.77550 \mathrm{E}+02$ & $8.49160 \mathrm{E}-06$ & 2.45410E.08 & $3.46460 \mathrm{E}-08$ & $3.45498 \mathrm{E}-09$ & $1.04815 \mathrm{E}-08$ & $3,38803 \mathrm{E}, 08$ & $3,37150 \mathrm{E}-08$ \\
\hline 33 & $9.00000 \mathrm{E}+00$ & $5.74370 \mathrm{E}+02$ & $9.86148 \mathrm{P} 0$ & 2,64246008 & $4.01542 \mathrm{E}-08$ & $3.65022 \mathrm{E}-09$ & $1.20881 \mathrm{E}-08$ & $3.92404 \mathrm{E}-08$ & $3.90512 \mathrm{E}-08$ \\
\hline 34 & $0.00000 E+00$ & $5.74370 E+02$ & Aotourtikos & 30,20801000 & $4.10978 \mathrm{E}-08$ & $3.65627 \mathrm{~F}=09$ & $123575 E_{0} 08$ & $4.01569 \mathrm{E}-08$ & $3.92635 \mathrm{E}-08$ \\
\hline
\end{tabular}

2Detoctor coordinates are relative to DORT geometry model.
bGamma-Ray dose units are (rem/hour)/(curie). 


\subsection{UNCERTAINTIES}

There are several areas where the uncertainties in the computational model, source distribution, and cross section data may cause significant perturbations to the reported results. An estimate of the magnitude of these perturbations is as follows:

1) The sources are assumed to be uniformly distributed throughout the cavity of the casks and not in isolated positions within the cavity. Possible error: increase in dose of a factor of 10 .

2) Due to lack of information on the composition of the source material, it is assumed to be void; thus there is no self-absorption within the source region of the computational model. Possible error: decrease of dose of a factor of 10.

3) The ORIGEN-S code is a point-depletion code; thus, it assumes that all points in the reactor see the same flux/power. Possible error: small decrease of dose.

4) The ORIGEN-S calculations assume the cross-section library is appropriate for the situation. For the two PWR cases, one-dimensional radiation transport calculations were made with a broad-group library, then were collapsed to the ORIGEN-S group structure; thus, the PWR ORIGEN-S calculations should be very good. For the ORR cases, the same PWR ORIGEN-S cross-section libraries were used; this will inherently include some uncertainty in the results. Possible error: increase in dose of a factor of 2.

5) The DORT radiation transport calculations used Legendre $P_{5}$ cross sections. Possible error: negligible increase in dose.

In consideration of the possible contributors to the calculational uncertainties listed above, the first two dealing with the source descriptions within the calculational models represent the two largest uncertainties. It should be noted that they are estimated to be equal in magnitude and may essentially negate each other for a given calculation. 


\subsection{EXAMPLE APPLICATIONS OF DOSE AND THERMAL HEATING DATA TABLES}

The dose and heating rate data presented in Tables 6 - 12 of this report are reported on a per-curie basis, so cask and SNF source dependent expected dose and heating rates can be readily calculated for all the projected sequence of moves for storage facility 7827 in the SWSA. To demonstrate the application of the tabulated dose and heating rate data, two examples are given below.

Example 1: Transfer of container with Accountability Transfer Number (ATN) 1802 from Well 5 to Well 1. This SNF package contains sections of an H.B. Robinson fuel assembly which was estimated to contain 1,200 Ci of MFP when it was loaded into Well 5 in April 1979. The cask identified in the Transportation Plan to accommodate this move is the Loop Transport Carrier. To be conservative, assume there is no reduction in the source strength, whereas in reality, the source has decayed to a lower value (Figure 7 can be used to determine actual source strength, if desired). Furthermore, assume the source has a high burnup rate and use the LWR MFP ( $50 \mathrm{MWd} / \mathrm{kgU}$ ) source data which will yield the higher doses and heating rates. From Table 6 , the alpha and beta heating rate is determined to be $3.24 \mathrm{~W}[2.70 \mathrm{E}-3 \mathrm{~W} / \mathrm{Ci} \times 1,200 \mathrm{Ci}\}]$. From Table 7, the photon heating rate is determined to be $1.10 \mathrm{~W}$. Therefore, the total thermal load on the cask for this SNF package is $4.34 \mathrm{~W}$. To determine the maximum dose rates on the surface and at one meter from the surface of the cask, look in Table 8 under the $50 \mathrm{MWd} / \mathrm{kgU}$ LWR MFP source column and find the maximum dose rates. For the surface dose, this occurs at detector 17, and for the one meter distance dose, this occurs at detector 25. Multiply the dose rates (per curie) from Table 8 at these two detector locations by the number of source curies to yield the maximum expected dose rate for this SNF package in this cask. This yields a surface dose rate of $9.66 \mathrm{E}-02$ $\mathrm{mrem} / \mathrm{h}$ and a one meter distance dose rate of 5.23E-03 mrem/h. From these dose rates and heating rates, this SNF package can safely be transported using this cask and not exceed the transportation guidelines.

In the above analysis, the MFP source strength was not decayed to the level which would be present after being stored in the well for 16 years. This represents a conservative approach and will always yield higher heating rates and dose rates than will actually occur. To be more precise, the above analysis should be repeated with the source strength corrected using Figure 7 to account for decay. 
Example 2: Transfer of ATN 1340 from Well 1 to Well 26. This SNF package contains irradiated stainless steel clad with fuel removed and is estimated to contain approximately $1,000 \mathrm{Ci}$ of ${ }^{60} \mathrm{Co}$ when it was loaded in the well in December 1976. The cask identified in the Transportation Plan to accommodate this move is the 6.5 -Inch HRLEL Carrier. ${ }^{60} \mathrm{Co}$ has a 5.27 year half life. Assuming the package will be moved in June 1995, the source strength has decayed to $87.8 \mathrm{Ci}\left[\mathrm{N}=\mathrm{N} 0 * \exp \left((-0.693 / 5.27)^{*} 18.5\right)\right]$ where $\mathrm{N}$ is the source strength in June 1995, and N0 is the initial source strength back in December 1976. From Table 6, the alpha and beta heating rate is $5.066 \mathrm{E}-02 \mathrm{~W}$, and from Table 7, the photon heating rate is $1.295 \mathrm{~W}$. This gives a total heating rate of $1.35 \mathrm{~W}$, which is well within the Transportation Plan guidelines. To obtain the maximum dose rates on the surface and at one meter from the surface, look in Table 10 under the ${ }^{60} \mathrm{Co}$ source column. Detector 1 yields the highest dose rate of $34.61 \mathrm{mrem} / \mathrm{h}$ on the surface of the cask, and Detector 18 yields the highest dose rate of $1.52 \mathrm{mrem} / \mathrm{h}$ at one meter distance from the cask surface. Again these dose rates are within the guidelines presented in the Transportation Plan.

The procedure demonstrated in the above two examples is applicable to all of the SNF packages to be transported. In those cases where there is a combination of sources (i.e., ${ }^{60} \mathrm{Co}$ and MFP) then the above analysis should be performed for each source and the results combined to obtain the total heating and dose rates. 


\subsection{REFERENCES}

1. C. Y. Fu and D. T. Ingersoll. "VELM61 and VELM22: Multigroup Cross-Section Libraries for Sodium-Cooled Reactor Shield Analysis," ORNL/TM-10302, April 1987.

2. C. R. Weisbin, R. W. Roussin, J. Wagschal, J. E. White, and R. Q. Wright. "VITAMIN-E: An ENDF/B-V Multigroup Cross-Section Library for LMFBR Core and Shield, LWR Shield, Dosimetry and Fussion-Blanket Technology," ORNL-5505 (ENDF-274), 1979. The release by RSIC, dated August 1985, is denoted as DLC-133B.

3. W. A. Rhoades and M. B. Emmett, "DOS: The Discrete Ordinates System," ORNL/TM-8362, September 1982.

4. N. M. Greene, W. E. Ford III, L. M. Petrie, and J. W. Arwood. "AMPX-77: A Modular Code System for Generating Coupled Multigroup Neutron-Gamma Cross-Section Libraries from ENDF/B-IV and/or ENDF/B-V," ORNL/CSD/TM-283, October 1992.

5. G. C. Haynes and W. W. Engle. "AMP - Activity Manipulation Program," ORNL-CF75-6-53, June 1975.

6. O. W. Hermann, C. V. Parks, and J. P. Renier. "Technical Support for a Proposed Decay Heat Guide Using SAS2H/ORIGEN-S Data," NUREG/CR-5625 (ORNL6698), September 1994.

7. J. A. Klein maintains the SNF Database at ORNL.

8. O. W. Hermann and R. M. Westfall. "ORIGEN-S: SCALE System Module to Calculate Fuel Depletion, Actinide Transmutation, Fission Product Buildup and Decay, and Associated Radiation Source Terms," as described in Sect. F7 of SCALE: A Modular Code System for Performing Standardized Computer Analyses for Licensing Evaluation, NUREG/CR-0200, Rev. 4 (ORNL/NUREG/CSD-2/R4), Vols. I-III. Available from Radiation Shielding Information Center at Oak Ridge National Laboratory as CCC-545.

9. R. L. Sean. "Summary Report on the HFED Miniplate Irradiations for the RERTR Program," ORNL-6539, April 1989.

10. W. A Rhoades and R. L. Childs, "The DORT Two-Dimensional Discrete Ordinates Transport Code,"Nuclear Science \& Engineering 99, 1, 88-89 (May 1988).

11. D. T. Ingersoll and C. O. Slater, "DOGS - A Collection of Graphics For Support Of Discrete Ordinates Codes," ORNL/TM-7188, March 1980.

12. R. L. Childs, "The FAISTF Last-Flight Computer Program," ORNL/TM-12675, to be published.

13. ANSI/ANS-6.1.1-1977, American National Standard Neutron and Gamma-Ray. Flux-to-Dose-Rate Factors (1977). 

APPENDIX A

Gamma Source Spectra 

Table A.1 Gamma Source Spectra (gammas/s.Ci), VELM 23 Grp, 25MWd/kgU, 18kW/kgU, Continuous Operation = 1388.9d

\begin{tabular}{|c|c|c|c|c|c|c|c|c|c|c|c|}
\hline \multicolumn{12}{|c|}{ Decay Period } \\
\hline \multicolumn{2}{|c|}{$\begin{array}{l}\text { Energy Interval } \\
(\mathrm{MeV})\end{array}$} & \multirow{2}{*}{$\frac{1 \mathrm{Y}}{3.65 \mathrm{E}+09}$} & \multirow{2}{*}{$\frac{5 \mathrm{Y}}{1.83 \mathrm{E}+09}$} & \multirow{2}{*}{$\frac{10 \mathrm{Y}}{1.64 \mathrm{E}+09}$} & \multirow{2}{*}{$\frac{15 \mathrm{Y}}{1.72 \mathrm{E}+09}$} & \multirow{2}{*}{$\frac{20 \mathrm{Y}}{1.79 \mathrm{E}+09}$} & \multirow{2}{*}{$\frac{25 \mathrm{Y}}{1.85 \mathrm{E}+09}$} & \multirow{2}{*}{$\frac{30 \mathrm{Y}}{1.90 \mathrm{E}+09}$} & \multirow{2}{*}{$\frac{35 \mathrm{Y}}{1.95 \mathrm{E}+09}$} & \multirow{2}{*}{$\frac{40 \mathrm{Y}}{2.01 \mathrm{E}+09}$} & \multirow{2}{*}{$\frac{45 Y}{2.05 E+09}$} \\
\hline $1.00 \mathrm{E}-02$ & $2.00 \mathrm{E}-02$ & & & & & & & & & & \\
\hline $2.00 \mathrm{E}-02$ & $4.50 \mathrm{E}-02$ & $4.50 \mathrm{E}+09$ & $2.38 \mathrm{E}+09$ & $2.12 \mathrm{E}+09$ & $2.15 \mathrm{E}+09$ & $2.18 \mathrm{E}+09$ & $2.21 \mathrm{E}+09$ & $2.24 \mathrm{E}+09$ & $2.27 \mathrm{E}+09$ & $2.30 \mathrm{E}+09$ & $2.32 \mathrm{E}+09$ \\
\hline $4.50 \mathrm{E}-02$ & $7.00 \mathrm{E}-02$ & $1.78 \mathrm{E}+09$ & $8.74 E+08$ & $8.03 E+08$ & $8.70 \mathrm{E}+08$ & $9.33 \mathrm{E}+08$ & $9.94 \mathrm{E}+08$ & $1.06 \mathrm{E}+09$ & $1.13 E+09$ & $1.20 \mathrm{E}+09$ & $1.27 \mathrm{E}+09$ \\
\hline $7.00 \mathrm{E}-02$ & $1.00 \mathrm{E}-01$ & $1.42 \mathrm{E}+09$ & $6.29 E+08$ & $5.13 E+08$ & $5.16 \mathrm{E}+08$ & $5.20 \mathrm{E}+08$ & $5.24 \mathrm{E}+08$ & $5.31 \mathrm{E}+08$ & $5.35 \mathrm{E}+08$ & $5.42 \mathrm{E}+08$ & $5.46 \mathrm{E}+08$ \\
\hline $1.00 \mathrm{E}-01$ & $1.50 \mathrm{E}-01$ & $1.87 \mathrm{E}+09$ & $7.10 \mathrm{E}+08$ & $5.45 \mathrm{E}+08$ & $5.22 \mathrm{E}+08$ & $5.03 \mathrm{E}+08$ & $4.88 \mathrm{E}+08$ & $4.78 \mathrm{E}+08$ & $4.69 E+08$ & $4.66 \mathrm{E}+08$ & $4.61 \mathrm{E}+08$ \\
\hline $1.50 \mathrm{E}-01$ & $3.00 \mathrm{E}-01$ & $1.29 \mathrm{E}+09$ & $5.40 \mathrm{E}+08$ & $4.34 \mathrm{E}+08$ & $4.43 E+08$ & $4.50 \mathrm{E}+08$ & $4.55 \mathrm{E}+08$ & $4.60 \mathrm{E}+08$ & $4.63 \mathrm{E}+08$ & $4.68 \mathrm{E}+08$ & $4.69 \mathrm{E}+08$ \\
\hline $3.00 \mathrm{E}-01$ & $4.00 \mathrm{E}-01$ & $4.87 E+08$ & $1.81 E+08$ & $1.37 \mathrm{E}+08$ & $1.41 \mathrm{E}+08$ & $1.45 \mathrm{E}+08$ & $1.48 \mathrm{E}+08$ & $1.51 \mathrm{E}+08$ & $1.52 \mathrm{E}+08$ & $1.54 \mathrm{E}+08$ & $1.55 \mathrm{E}+08$ \\
\hline $4.00 \mathrm{E}-01$ & $5.10 \mathrm{E}-01$ & $9.13 E+08$ & $3.00 \mathrm{E}+08$ & $1.14 \mathrm{E}+08$ & $9.24 \mathrm{E}+07$ & $8.81 E+07$ & $8.74 \mathrm{E}+07$ & $8.81 \mathrm{E}+07$ & $8.86 \mathrm{E}+07$ & $8.96 \mathrm{E}+07$ & $8.99 \mathrm{E}+07$ \\
\hline $5.10 \mathrm{E}-01$ & $6.00 \mathrm{E}-01$ & $8.67 E+08$ & $4.80 \mathrm{E}+08$ & $1.36 \mathrm{E}+08$ & $5.85 \mathrm{E}+07$ & $3.84 \mathrm{E}+07$ & $3.16 \mathrm{E}+07$ & $2.84 \mathrm{E}+07$ & $2.62 E+07$ & $2.48 \mathrm{E}+07$ & $2.36 \mathrm{E}+07$ \\
\hline $6.00 \mathrm{E}-01$ & $7.00 \mathrm{E}-01$ & $3.68 \mathrm{E}+09$ & $6.49 E+09$ & $6.76 \mathrm{E}+09$ & $6.96 \mathrm{E}+09$ & $7.19 \mathrm{E}+09$ & $7.38 \mathrm{E}+09$ & $7.58 \mathrm{E}+09$ & $7.73 \mathrm{E}+09$ & $7.91 \mathrm{E}+09$ & $8.01 \mathrm{E}+09$ \\
\hline $7.00 \mathrm{E}-01$ & $1.00 \mathrm{E}+00$ & $2.65 \mathrm{E}+09$ & $1.43 \mathrm{E}+09$ & $5.27 E+08$ & $2.52 \mathrm{E}+08$ & $1.66 \mathrm{E}+08$ & $1.29 \mathrm{E}+08$ & $1.06 \mathrm{E}+08$ & $8.97 \mathrm{E}+07$ & $7.77 \mathrm{E}+07$ & $6.81 \mathrm{E}+07$ \\
\hline $1.00 \mathrm{E}+00$ & $1.50 \mathrm{E}+00$ & $3.33 \mathrm{E}+08$ & $3.05 \mathrm{E}+08$ & $2.10 \mathrm{E}+08$ & $1.59 \mathrm{E}+08$ & $1.24 \mathrm{E}+08$ & $9.82 \mathrm{E}+07$ & $7.86 \mathrm{E}+07$ & $6.30 \mathrm{E}+07$ & $5.14 \mathrm{E}+07$ & $4.21 \mathrm{E}+07$ \\
\hline $1.50 \mathrm{E}+00$ & $2.00 \mathrm{E}+00$ & $4.03 E+07$ & $1.41 \mathrm{E}+07$ & $7.85 \mathrm{E}+06$ & $6.36 \mathrm{E}+06$ & $5.20 \mathrm{E}+06$ & $4.26 \mathrm{E}+06$ & $3.54 \mathrm{E}+06$ & $2.97 \mathrm{E}+06$ & $2.54 \mathrm{E}+06$ & $2.19 \mathrm{E}+06$ \\
\hline $2.00 \mathrm{E}+00$ & $2.50 \mathrm{E}+00$ & $5.66 \mathrm{E}+07$ & $6.54 \mathrm{E}+06$ & $1.77 \mathrm{E}+05$ & $2.25 \mathrm{E}+04$ & $1.89 \mathrm{E}+04$ & $1.91 \mathrm{E}+04$ & $1.95 \mathrm{E}+04$ & $1.97 \mathrm{E}+04$ & $2.00 \mathrm{E}+04$ & $2.01 \mathrm{E}+04$ \\
\hline $2.50 \mathrm{E}+00$ & $3.00 \mathrm{E}+00$ & $9.84 \mathrm{E}+05$ & $2.16 \mathrm{E}+05$ & $1.11 \mathrm{E}+04$ & $1.93 \mathrm{E}+03$ & $1.79 \mathrm{E}+03$ & $1.97 \mathrm{E}+03$ & $2.17 \mathrm{E}+03$ & $2.36 \mathrm{E}+03$ & $2.58 \mathrm{E}+03$ & $2.79 \mathrm{E}+03$ \\
\hline $3.00 \mathrm{E}+00$ & $4.00 \mathrm{E}+00$ & $1.19 \mathrm{E}+05$ & $2.69 \mathrm{E}+04$ & $1.29 \mathrm{E}+03$ & $8.08 \mathrm{E}+01$ & $3.19 \mathrm{E}+01$ & $2.90 \mathrm{E}+01$ & $2.80 \mathrm{E}+01$ & $2.69 \mathrm{E}+01$ & $2.60 \mathrm{E}+01$ & $2.50 \mathrm{E}+01$ \\
\hline $4.00 \mathrm{E}+00$ & $5.00 \mathrm{E}+00$ & $4.24 \mathrm{E}+\infty$ & $8.83 \mathrm{E}+00$ & $1.03 \mathrm{E}+01$ & $1.04 \mathrm{E}+01$ & $1.01 \mathrm{E}+01$ & $9.75 \mathrm{E}+00$ & $9.42 \mathrm{E}+00$ & $9.06 \mathrm{E}+00$ & $8.76 \mathrm{E}+00$ & $8.43 \mathrm{E}+00$ \\
\hline $5.00 \mathrm{E}+00$ & $6.00 \mathrm{E}+00$ & $1.43 \mathrm{E}+\infty$ & $2.98 \mathrm{E}+00$ & $3.48 \mathrm{E}+00$ & $3.49 \mathrm{E}+00$ & $3.40 \mathrm{E}+00$ & $3.28 \mathrm{E}+00$ & $3.17 \mathrm{E}+00$ & $3.05 \mathrm{E}+00$ & $2.95 \mathrm{E}+00$ & $2.83 \mathrm{E}+00$ \\
\hline $6.00 \mathrm{E}+00$ & $7.00 \mathrm{E}+00$ & $4.81 \mathrm{E}-01$ & $1.00 \mathrm{E}+00$ & $1.17 \mathrm{E}+00$ & $1.18 \mathrm{E}+00$ & $1.15 \mathrm{E}+00$ & $1.11 \mathrm{E}+00$ & $1.07 \mathrm{E}+\infty$ & $1.03 \mathrm{E}+00$ & $9.91 \mathrm{E}-01$ & $9.53 \mathrm{E}-01$ \\
\hline $7.00 \mathrm{E}+00$ & $7.50 \mathrm{E}+00$ & $1.03 \mathrm{E}-01$ & $2.15 \mathrm{E}-01$ & $2.52 \mathrm{E}-01$ & $2.53 \mathrm{E}-01$ & $2.46 \mathrm{E}-01$ & $2.38 \mathrm{E}-01$ & $2.29 \mathrm{E}-01$ & $2.20 \mathrm{E}-01$ & $2.13 \mathrm{E}-01$ & 2.05E-01 \\
\hline $7.50 \mathrm{E}+00$ & $8.00 \mathrm{E}+00$ & $6.00 \mathrm{E}-02$ & $1.25 \mathrm{E}-01$ & $1.46 \mathrm{E}-01$ & $1.46 \mathrm{E}-01$ & $1.43 \mathrm{E}-01$ & $1.38 \mathrm{E}-01$ & $1.33 \mathrm{E}-01$ & $1.28 \mathrm{E}-01$ & $1.23 \mathrm{E}-01$ & $1.19 \mathrm{E}-01$ \\
\hline $8.00 \mathrm{E}+00$ & $1.00 \mathrm{E}+01$ & $7.08 \mathrm{E}-02$ & $1.48 \mathrm{E}-01$ & $1.73 \mathrm{E}-01$ & $1.73 \mathrm{E}-01$ & $1.68 \mathrm{E}-01$ & $1.63 \mathrm{E}-01$ & $1.57 \mathrm{E}-01$ & $1.51 \mathrm{E}-01$ & $1.46 \mathrm{E}-01$ & $1.40 \mathrm{E}-01$ \\
\hline $1.00 \mathrm{E}+01$ & $1.40 \mathrm{E}+01$ & $3.36 \mathrm{E}-03$ & $6.99 \mathrm{E}-03$ & $8.18 \mathrm{E}-03$ & $8.19 \mathrm{E}-03$ & $7.98 \mathrm{E}-03$ & $7.70 \mathrm{E}-03$ & $7.43 \mathrm{E}-03$ & $7.14 \mathrm{E}-03$ & $6.89 \mathrm{E}-03$ & $6.62 \mathrm{E}-03$ \\
\hline & & & & & & & & & & & \\
\hline Totals & & $2.35 \mathrm{E}+10$ & $1.62 \mathrm{E}+10$ & $1.40 \mathrm{E}+10$ & $1.39 \mathrm{E}+10$ & $1.41 E+10$ & $1.44 \mathrm{E}+10$ & $1.47 \mathrm{E}+10$ & $1.50 \mathrm{E}+10$ & $1.53 \mathrm{E}+10$ & $1.55 \mathrm{E}+10$ \\
\hline
\end{tabular}


Table A.2 Gamma Source Spectra (gammas/s.Ci), VELM 23 Grp, 50MWd/kgU, 40kW/kgU, Continuous Operation = 1250d

\begin{tabular}{|c|c|c|c|c|c|c|c|c|c|}
\hline \multicolumn{10}{|c|}{ Decay Period } \\
\hline \multicolumn{2}{|c|}{$\begin{array}{c}\text { Energy Interval } \\
(\mathrm{MeV})\end{array}$} & $1 Y$ & $5 Y$ & $10 Y$ & $15 \mathrm{Y}$ & $20 Y$ & $25 \mathrm{Y}$ & $30 Y$ & $35 Y$ \\
\hline $1.00 \mathrm{E}-02$ & $2.00 \mathrm{E}-02$ & $3.76 \mathrm{E}+09$ & $1.95 \mathrm{E}+09$ & $1.73 \mathrm{E}+09$ & $1.81 \mathrm{E}+09$ & $1.87 \mathrm{E}+09$ & $1.92 \mathrm{E}+09$ & $1.97 \mathrm{E}+09$ & $2.02 \mathrm{E}+09$ \\
\hline $2.00 \mathrm{E}-02$ & $4.50 \mathrm{E}-02$ & $4.63 \mathrm{E}+09$ & $2.56 \mathrm{E}+09$ & $2.25 \mathrm{E}+09$ & $2.26 \mathrm{E}+09$ & $2.27 \mathrm{E}+09$ & $2.29 E+09$ & $2.31 \mathrm{E}+09$ & $2.33 \mathrm{E}+09$ \\
\hline $4.50 \mathrm{E}-02$ & $7.00 \mathrm{E}-02$ & $1.84 \mathrm{E}+09$ & $9.24 \mathrm{E}+08$ & $8.27 E+08$ & $8.81 E+08$ & $9.32 \mathrm{E}+08$ & $9.83 \mathrm{E}+08$ & $1.04 \mathrm{E}+09$ & $1.09 \mathrm{E}+09$ \\
\hline $7.00 \mathrm{E}-02$ & $1.00 \mathrm{E}-01$ & $1.47 \mathrm{E}+09$ & $6.93 \mathrm{E}+08$ & $5.54 \mathrm{E}+08$ & $5.45 \mathrm{E}+08$ & $5.42 \mathrm{E}+08$ & $5.43 \mathrm{E}+08$ & $5.46 \mathrm{E}+08$ & $5.50 \mathrm{E}+08$ \\
\hline $1.00 \mathrm{E}-01$ & $1.50 \mathrm{E}-01$ & $1.95 \mathrm{E}+09$ & $8.26 \mathrm{E}+08$ & $6.39 \mathrm{E}+08$ & $5.95 \mathrm{E}+08$ & $5.60 \mathrm{E}+08$ & $5.33 \mathrm{E}+08$ & $5.14 \mathrm{E}+08$ & $4.99 \mathrm{E}+08$ \\
\hline $1.50 \mathrm{E}-01$ & $3.00 \mathrm{E}-01$ & $1.32 \mathrm{E}+09$ & $5.81 \mathrm{E}+08$ & $4.61 \mathrm{E}+08$ & $4.66 \mathrm{E}+08$ & $4.70 \mathrm{E}+08$ & $4.73 \mathrm{E}+08$ & $4.76 \mathrm{E}+08$ & $4.78 \mathrm{E}+08$ \\
\hline $3.00 \mathrm{E}-01$ & $4.00 \mathrm{E}-01$ & $5.00 \mathrm{E}+08$ & $1.91 \mathrm{E}+08$ & $1.42 \mathrm{E}+08$ & $1.46 \mathrm{E}+08$ & $1.49 \mathrm{E}+08$ & $1.52 \mathrm{E}+08$ & $1.54 \mathrm{E}+08$ & $1.56 \mathrm{E}+08$ \\
\hline $4.00 \mathrm{E}-01$ & $5.10 \mathrm{E}-01$ & $9.57 \mathrm{E}+08$ & $3.37 \mathrm{E}+08$ & $1.24 \mathrm{E}+08$ & $9.71 \mathrm{E}+07$ & $9.15 \mathrm{E}+07$ & $9.02 \mathrm{E}+07$ & $9.05 \mathrm{E}+07$ & $9.09 \mathrm{E}+07$ \\
\hline $5.10 \mathrm{E}-01$ & $6.00 \mathrm{E}-01$ & $1.10 \mathrm{E}+09$ & $7.25 \mathrm{E}+08$ & $2.09 \mathrm{E}+08$ & $8.13 E+07$ & $4.85 \mathrm{E}+07$ & $3.78 \mathrm{E}+07$ & $3.28 \mathrm{E}+07$ & $2.97 \mathrm{E}+07$ \\
\hline $6.00 \mathrm{E}-01$ & $7.00 \mathrm{E}-01$ & $4.40 \mathrm{E}+09$ & $7.40 \mathrm{E}+09$ & $7.20 \mathrm{E}+09$ & $7.23 \mathrm{E}+09$ & $7.40 \mathrm{E}+09$ & $7.58 \mathrm{E}+09$ & $7.77 \mathrm{E}+09$ & $7.92 \mathrm{E}+09$ \\
\hline $7.00 \mathrm{E}-01$ & $1.00 \mathrm{E}+00$ & $3.47 \mathrm{E}+09$ & $2.32 \mathrm{E}+09$ & $8.35 \mathrm{E}+08$ & $3.71 \mathrm{E}+08$ & $2.31 \mathrm{E}+08$ & $1.73 \mathrm{E}+08$ & $1.39 \mathrm{E}+08$ & $1.14 \mathrm{E}+08$ \\
\hline $1.00 \mathrm{E}+00$ & $1.50 \mathrm{E}+00$ & $4.19 \mathrm{E}+08$ & $4.44 \mathrm{E}+08$ & $3.07 \mathrm{E}+08$ & $2.28 \mathrm{E}+08$ & $1.76 \mathrm{E}+08$ & $1.38 \mathrm{E}+08$ & $1.09 \mathrm{E}+08$ & $8.60 \mathrm{E}+07$ \\
\hline $1.50 \mathrm{E}+00$ & $2.00 \mathrm{E}+00$ & $4.34 \mathrm{E}+07$ & $1.79 \mathrm{E}+07$ & $1.10 \mathrm{E}+07$ & $8.85 \mathrm{E}+06$ & $7.14 \mathrm{E}+06$ & $5.76 \mathrm{E}+06$ & $4.68 \mathrm{E}+06$ & $3.85 \mathrm{E}+06$ \\
\hline $2.00 \mathrm{E}+00$ & $2.50 \mathrm{E}+00$ & $5.81 \mathrm{E}+07$ & $7.23 \mathrm{E}+06$ & $1.99 \mathrm{E}+05$ & $2.37 \mathrm{E}+04$ & $1.95 \mathrm{E}+04$ & $1.97 \mathrm{E}+04$ & $2.00 \mathrm{E}+04$ & $2.03 \mathrm{E}+04$ \\
\hline $2.50 \mathrm{E}+00$ & $3.00 \mathrm{E}+00$ & $1.03 \mathrm{E}+06$ & $2.43 E+05$ & $1.43 \mathrm{E}+04$ & $4.33 \mathrm{E}+03$ & $4.50 \mathrm{E}+03$ & $4.98 \mathrm{E}+03$ & $5.46 \mathrm{E}+03$ & $5.95 \mathrm{E}+03$ \\
\hline $3.00 \mathrm{E}+00$ & $4.00 \mathrm{E}+00$ & $1.25 \mathrm{E}+05$ & $3.02 \mathrm{E}+04$ & $1.55 \mathrm{E}+03$ & $1.73 \mathrm{E}+02$ & $1.14 \mathrm{E}+02$ & $1.07 \mathrm{E}+02$ & $1.03 \mathrm{E}+02$ & $9.78 \mathrm{E}+01$ \\
\hline $4.00 \mathrm{E}+00$ & $5.00 \mathrm{D}+00$ & $1.18 \mathrm{E}+01$ & $3.31 \mathrm{E}+01$ & $3.93 \mathrm{E}+01$ & $3.91 \mathrm{E}+01$ & $3.78 \mathrm{E}+01$ & $3.62 \mathrm{E}+01$ & $3.46 \mathrm{E}+01$ & $3.30 \mathrm{E}+01$ \\
\hline $5.00 \mathrm{E}+00$ & $6.00 \mathrm{E}+00$ & $3.98 \mathrm{E}+00$ & $1.11 \mathrm{E}+01$ & $1.32 \mathrm{E}+01$ & $1.32 \mathrm{E}+01$ & $1.27 \mathrm{E}+01$ & $1.22 \mathrm{E}+01$ & $1.17 \mathrm{E}+01$ & $1.11 \mathrm{E}+01$ \\
\hline $6.00 \mathrm{E}+00$ & $7.00 \mathrm{E}+00$ & $1.34 \mathrm{E}+00$ & $3.75 \mathrm{E}+00$ & $4.46 \mathrm{E}+00$ & $4.44 \mathrm{E}+00$ & $4.29 \mathrm{E}+00$ & $4.11 \mathrm{E}+00$ & $3.93 \mathrm{E}+00$ & $3.74 \mathrm{E}+00$ \\
\hline $7.00 \mathrm{E}+00$ & $7.50 \mathrm{E}+00$ & $2.88 \mathrm{E}-01$ & 8.07E-01 & $9.58 \mathrm{E}-01$ & $9.54 \mathrm{E}-01$ & $9.23 \mathrm{E}-01$ & $8.84 \mathrm{E}-01$ & $8.45 \mathrm{E}-01$ & 8.05E-01 \\
\hline $7.50 \mathrm{E}+00$ & $8.00 \mathrm{E}+00$ & $1.67 \mathrm{E}-01$ & $4.68 \mathrm{E}-01$ & $5.56 \mathrm{E}-01$ & $5.53 \mathrm{E}-01$ & 5.35E-01 & $5.12 \mathrm{E}-01$ & $4.90 \mathrm{E}-01$ & $4.67 \mathrm{E}-01$ \\
\hline $8.00 \mathrm{E}+00$ & $1.00 \mathrm{E}+01$ & $1.97 \mathrm{E}-01$ & $5.52 \mathrm{E}-01$ & $6.56 \mathrm{E}-01$ & $6.53 \mathrm{E}-01$ & $6.32 \mathrm{E}-01$ & $6.05 \mathrm{E}-01$ & $5.79 \mathrm{E}-01$ & $5.51 \mathrm{E}-01$ \\
\hline $1.00 \mathrm{E}+01$ & $1.40 \mathrm{E}+01$ & $9.36 \mathrm{E}-03$ & $2.62 \mathrm{E}-02$ & $3.11 \mathrm{E}-02$ & $3.10 \mathrm{E}-02$ & $2.99 \mathrm{E}-02$ & $2.87 \mathrm{E}-02$ & $2.74 \mathrm{E}-02$ & $2.61 E-02$ \\
\hline Totals & & $2.59 E+10$ & $1.90 \mathrm{E}+10$ & $1.53 \mathrm{E}+10$ & $1.47 \mathrm{E}+10$ & $1.47 \mathrm{E}+10$ & $1.49 \mathrm{E}+10$ & $1.51 \mathrm{E}+10$ & $1.54 \mathrm{E}+10$ \\
\hline
\end{tabular}


Table A.3 Gamma Source Spectra (gammas/s.Ci), VELM 23 Grp. HFED UAl, Module 1, Slot 1, 40.24\% Enrichment, 94\% Depleted, 7.65E-4 MW/gU

\begin{tabular}{|c|c|c|c|c|c|c|c|c|c|c|c|}
\hline \multicolumn{12}{|c|}{ Decay Period } \\
\hline \multicolumn{2}{|c|}{$\begin{array}{c}\text { Energy Interval } \\
(\mathrm{MeV})\end{array}$} & $1 Y$ & $5 Y$ & $10 \mathrm{Y}$ & $15 Y$ & $20 Y$ & $25 Y$ & $30 \mathrm{Y}$ & $35 Y$ & $40 \mathrm{Y}$ & $45 Y$ \\
\hline $1.00 \mathrm{E}-02$ & $2.00 \mathrm{E}-02$ & $4.01 E+09$ & $2.59 \mathrm{E}+09$ & $2.41 \mathrm{E}+09$ & $2.46 \mathrm{E}+09$ & $2.49 \mathrm{E}+09$ & $2.50 \mathrm{E}+09$ & $2.51 \mathrm{E}+09$ & $2.51 \mathrm{E}+09$ & $2.51 E+09$ & $2.51 \mathrm{E}+09$ \\
\hline $2.00 \mathrm{E}-02$ & $4.50 \mathrm{E}-02$ & $5.17 \mathrm{E}+09$ & $3.19 \mathrm{E}+09$ & $2.87 E+09$ & $2.86 \mathrm{E}+09$ & $2.84 \mathrm{E}+09$ & $2.83 E+09$ & $2.81 E+09$ & $2.79 \mathrm{E}+09$ & $2.77 \mathrm{E}+09$ & $2.77 \mathrm{E}+09$ \\
\hline $4.50 \mathrm{E}-02$ & $7.00 \mathrm{E}-02$ & $1.94 \mathrm{E}+09$ & $1.17 \mathrm{E}+09$ & $1.06 \mathrm{E}+09$ & $1.07 \mathrm{E}+09$ & $1.08 \mathrm{E}+09$ & $1.08 \mathrm{E}+09$ & $1.08 \mathrm{E}+09$ & $1.08 \mathrm{E}+09$ & $1.08 \mathrm{E}+09$ & $1.08 \mathrm{E}+09$ \\
\hline $7.00 \mathrm{E}-02$ & $1.00 \mathrm{E}-01$ & $1.61 \mathrm{E}+09$ & $8.85 E+08$ & $7.46 \mathrm{E}+08$ & $7.37 \mathrm{E}+08$ & $7.28 \mathrm{E}+08$ & $7.22 \mathrm{E}+08$ & $7.18 \mathrm{E}+08$ & $7.13 \mathrm{E}+08$ & $7.08 \mathrm{E}+08$ & $7.06 \mathrm{E}+08$ \\
\hline $1.00 \mathrm{E}-01$ & $1.50 \mathrm{E}-01$ & $2.35 E+09$ & $1.03 E+09$ & $7.75 \mathrm{E}+08$ & $7.33 \mathrm{E}+08$ & $6.95 \mathrm{E}+08$ & $6.65 E+08$ & $6.41 E+08$ & $6.21 E+08$ & $6.06 \mathrm{E}+08$ & $5.95 E+08$ \\
\hline $1.50 \mathrm{E}-01$ & $3.00 \mathrm{E}-01$ & $1.37 \mathrm{E}+09$ & $7.39 \mathrm{E}+08$ & $6.34 \mathrm{E}+08$ & $6.40 E+08$ & $6.38 \mathrm{E}+08$ & $6.35 E+08$ & $6.30 \mathrm{E}+08$ & $6.25 \mathrm{E}+08$ & $6.20 \mathrm{E}+08$ & $6.16 \mathrm{E}+08$ \\
\hline $3.00 \mathrm{E}-01$ & $4.00 \mathrm{E}-01$ & $5.16 \mathrm{E}+08$ & $2.47 \mathrm{E}+08$ & $2.02 \mathrm{E}+08$ & $2.07 \mathrm{E}+08$ & $2.08 E+08$ & $2.09 E+08$ & $2.09 \mathrm{E}+08$ & $2.08 \mathrm{E}+08$ & $2.07 \mathrm{E}+08$ & $2.06 \mathrm{E}+08$ \\
\hline $4.00 \mathrm{E}-01$ & $5.10 \mathrm{E}-01$ & $7.04 \mathrm{E}+08$ & $3.63 E+08$ & $1.65 E+08$ & $1.33 E+08$ & $1.26 \mathrm{E}+08$ & $1.23 \mathrm{E}+08$ & $1.22 \mathrm{E}+08$ & $1.21 \mathrm{E}+08$ & $1.20 \mathrm{E}+08$ & $1.20 \mathrm{E}+08$ \\
\hline $5.10 \mathrm{E}-01$ & $6.00 \mathrm{E}-01$ & $1.22 \mathrm{E}+09$ & $1.45 \mathrm{E}+09$ & $4.35 \mathrm{E}+08$ & $1.35 \mathrm{E}+08$ & $6.38 \mathrm{E}+07$ & $4.52 \mathrm{E}+07$ & $3.85 \mathrm{E}+07$ & $3.50 \mathrm{E}+07$ & $3.26 \mathrm{E}+07$ & $3.09 \mathrm{E}+07$ \\
\hline $6.00 \mathrm{E}-01$ & $7.00 \mathrm{E}-01$ & $5.07 \mathrm{E}+09$ & $1.04 \mathrm{E}+10$ & $8.74 \mathrm{E}+09$ & $8.06 \mathrm{E}+09$ & $7.98 \mathrm{E}+09$ & $8.02 \mathrm{E}+09$ & $8.07 \mathrm{E}+09$ & $8.10 \mathrm{E}+09$ & $8.12 \mathrm{E}+09$ & $8.16 \mathrm{E}+09$ \\
\hline $7.00 \mathrm{E}-01$ & $1.00 \mathrm{E}+00$ & $5.69 \mathrm{E}+09$ & $5.03 \mathrm{E}+09$ & $1.63 E+09$ & $5.37 \mathrm{E}+08$ & $2.60 \mathrm{E}+08$ & $1.75 \mathrm{E}+08$ & $1.37 \mathrm{E}+08$ & $1.14 \mathrm{E}+08$ & $9.75 \mathrm{E}+07$ & $8.55 E+07$ \\
\hline $1.00 \mathrm{E}+00$ & $1.50 \mathrm{E}+00$ & $4.23 E+08$ & $5.84 \mathrm{E}+08$ & $3.37 \mathrm{E}+08$ & $2.20 \mathrm{E}+08$ & $1.61 \mathrm{E}+08$ & $1.24 \mathrm{E}+08$ & $9.75 \mathrm{E}+07$ & $7.74 \mathrm{E}+07$ & $6.23 E+07$ & $5.10 \mathrm{E}+07$ \\
\hline $1.50 \mathrm{E}+00$ & $2.00 \mathrm{E}+00$ & $3.84 \mathrm{E}+07$ & $1.71 E+07$ & $1.03 E+07$ & $8.35 \mathrm{E}+06$ & $6.74 \mathrm{E}+06$ & $5.47 \mathrm{E}+06$ & $4.49 \mathrm{E}+06$ & $3.74 \mathrm{E}+06$ & $3.16 \mathrm{E}+06$ & $2.74 E+06$ \\
\hline $2.00 \mathrm{E}+00$ & $2.50 \mathrm{E}+00$ & $8.01 E+07$ & $1.32 \mathrm{E}+07$ & $3.07 \mathrm{E}+05$ & $3.27 \mathrm{E}+04$ & $2.75 E+04$ & $2.74 \mathrm{E}+04$ & $2.74 \mathrm{E}+04$ & $2.73 \mathrm{E}+04$ & $2.72 \mathrm{E}+04$ & $2.71 \mathrm{E}+04$ \\
\hline $2.50 \mathrm{E}+00$ & $3.00 \mathrm{E}+00$ & $5.40 \mathrm{E}+05$ & $1.82 \mathrm{E}+05$ & $1.90 \mathrm{E}+04$ & $1.41 \mathrm{E}+04$ & $1.59 \mathrm{E}+04$ & $1.75 \mathrm{E}+04$ & $1.89 \mathrm{E}+04$ & $2.03 \mathrm{E}+04$ & $2.17 \mathrm{E}+04$ & $2.33 E+04$ \\
\hline $3.00 \mathrm{E}+00$ & $4.00 \mathrm{E}+00$ & $6.01 \mathrm{E}+04$ & $2.15 \mathrm{E}+04$ & $1.28 \mathrm{E}+03$ & $2.26 \mathrm{E}+02$ & $1.73 \mathrm{E}+02$ & $1.61 \mathrm{E}+02$ & $1.53 \mathrm{E}+02$ & $1.45 \mathrm{E}+02$ & $1.38 \mathrm{E}+02$ & $1.32 \mathrm{E}+02$ \\
\hline $4.00 \mathrm{E}+00$ & $5.00 \mathrm{E}+00$ & $1.99 \mathrm{E}+01$ & $6.60 \mathrm{E}+01$ & $6.79 \mathrm{E}+01$ & $6.20 \mathrm{E}+01$ & $5.78 \mathrm{E}+01$ & $5.45 \mathrm{E}+01$ & $5.16 \mathrm{E}+01$ & $4.89 \mathrm{E}+01$ & $4.65 \mathrm{E}+01$ & $4.45 \mathrm{E}+01$ \\
\hline $5.00 \mathrm{E}+00$ & $6.00 \mathrm{E}+00$ & $6.71 E+00$ & $2.22 \mathrm{E}+01$ & $2.29 \mathrm{E}+01$ & $2.09 \mathrm{E}+01$ & $1.95 \mathrm{E}+01$ & $1.84 \mathrm{E}+01$ & $1.74 \mathrm{E}+01$ & $1.65 \mathrm{E}+01$ & $1.57 \mathrm{E}+01$ & $1.50 \mathrm{E}+01$ \\
\hline $6.00 E+00$ & $7.00 \mathrm{E}+00$ & $2.26 \mathrm{E}+00$ & $7.49 \mathrm{E}+00$ & $7.70 \mathrm{E}+00$ & $7.04 \mathrm{E}+00$ & $6.56 \mathrm{E}+00$ & $6.18 \mathrm{E}+00$ & $5.85 \mathrm{E}+00$ & $5.55 \mathrm{E}+00$ & $5.27 \mathrm{E}+00$ & $5.05 E+00$ \\
\hline $7.00 \mathrm{E}+00$ & $7.50 \mathrm{E}+00$ & $4.86 \mathrm{E}-01$ & $1.61 \mathrm{E}+\infty$ & $1.66 \mathrm{E}+00$ & $1.51 \mathrm{E}+00$ & $1.41 \mathrm{E}+00$ & $1.33 \mathrm{E}+00$ & $1.26 \mathrm{E}+00$ & $1.19 \mathrm{E}+00$ & $1.13 \mathrm{E}+\infty 0$ & $1.08 \mathrm{E}+00$ \\
\hline $7.50 \mathrm{E}+00$ & $8.00 \mathrm{E}+00$ & $2.82 \mathrm{E}-01$ & $9.34 \mathrm{E}-01$ & $9.60 \mathrm{E}-01$ & $8.78 \mathrm{E}-01$ & $8.17 \mathrm{E}-01$ & $7.70 \mathrm{E}-01$ & $7.29 \mathrm{E}-01$ & $6.91 \mathrm{E}-01$ & 6.57E-01 & $6.29 \mathrm{E}-01$ \\
\hline $8.00 E+00$ & $1.00 \mathrm{E}+01$ & 3.33E-01 & $1.10 \mathrm{E}+\infty$ & $1.13 \mathrm{E}+00$ & $1.04 \mathrm{E}+00$ & $9.65 \mathrm{E}-01$ & $9.10 \mathrm{E}-01$ & $8.62 \mathrm{E}-01$ & 8.17E-01 & $7.76 \mathrm{E}-01$ & $7.43 \mathrm{E}-01$ \\
\hline $1.00 \mathrm{E}+01$ & $1.40 \mathrm{E}+01$ & $1.58 \mathrm{E}-02$ & $5.23 \mathrm{E}-02$ & $5.38 \mathrm{E}-02$ & $4.91 \mathrm{E}-02$ & $4.57 \mathrm{E}-02$ & $4.31 \mathrm{E}-02$ & $4.08 \mathrm{E}-02$ & $3.87 \mathrm{E}-02$ & $3.68 \mathrm{E}-02$ & $3.52 \mathrm{E}-02$ \\
\hline Totals & & $3.02 \mathrm{E}+10$ & $2.78 \mathrm{E}+10$ & $2.00 \mathrm{E}+10$ & $1.78 E+10$ & $1.73 E+10$ & $1.71 \mathrm{E}+10$ & $1.71 E+10$ & $1.70 \mathrm{E}+10$ & $1.69 \mathrm{E}+10$ & $1.69 E+10$ \\
\hline
\end{tabular}


Table A.4 Gamma Source Spectra (gammas/s.Ci), VELM 23 Grp. HFED U3O8, Module 5, Slot 10, 93.21\% Enrichment, $80 \%$ Depleted, 2.65E-3 MW/gU

\begin{tabular}{|c|c|c|c|c|c|c|c|c|c|c|c|}
\hline \multicolumn{12}{|c|}{ Decay Period } \\
\hline Energy & nterval & $1 Y$ & $5 Y$ & $10 Y$ & $15 Y$ & $20 \mathrm{Y}$ & $25 Y$ & $30 Y$ & $35 Y$ & $40 Y$ & $45 \mathrm{Y}$ \\
\hline \multicolumn{2}{|c|}{$(\mathrm{MeV})$} & & & & & & & & & & \\
\hline & & & & & & & & & & & \\
\hline $1.00 \mathrm{E}-02$ & $2.00 \mathrm{E}-02$ & $3.95 \mathrm{E}+09$ & $2.66 \mathrm{E}+09$ & $2.51 \mathrm{E}+09$ & $2.57 \mathrm{E}+09$ & $2.58 \mathrm{E}+09$ & $2.59 \mathrm{E}+09$ & $2.59 \mathrm{E}+09$ & $2.59 \mathrm{E}+09$ & $2.59 \mathrm{E}+09$ & $2.58 \mathrm{E}+09$ \\
\hline $2.00 \mathrm{E}-02$ & $4.50 \mathrm{E}-02$ & $5.21 \mathrm{E}+09$ & $3.35 \mathrm{E}+09$ & $3.01 \mathrm{E}+09$ & $3.02 \mathrm{E}+09$ & $3.00 E+09$ & $2.97 \mathrm{E}+09$ & $2.96 \mathrm{E}+09$ & $2.94 \mathrm{E}+09$ & $2.92 \mathrm{E}+09$ & $2.91 E+09$ \\
\hline $4.50 \mathrm{E}-02$ & $7.00 \mathrm{E}-02$ & $1.91 \mathrm{E}+09$ & $1.23 \mathrm{E}+09$ & $1.13 E+09$ & $1.15 \mathrm{E}+09$ & $1.15 \mathrm{E}+09$ & $1.14 \mathrm{E}+09$ & $1.14 \mathrm{E}+09$ & $1.13 \mathrm{E}+09$ & $1.13 \mathrm{E}+09$ & $1.12 \mathrm{E}+09$ \\
\hline $7.00 \mathrm{E}-02$ & $1.00 \mathrm{E}-01$ & $1.60 \mathrm{E}+09$ & $9.32 \mathrm{E}+08$ & $7.97 \mathrm{E}+08$ & $7.93 \mathrm{E}+08$ & $7.83 \mathrm{E}+08$ & $7.75 \mathrm{E}+08$ & $7.70 \mathrm{E}+08$ & $7.65 \mathrm{E}+08$ & $7.60 \mathrm{E}+08$ & $7.55 \mathrm{E}+08$ \\
\hline $1.00 \mathrm{E}-01$ & $1.50 \mathrm{E}-01$ & $2.45 \mathrm{E}+09$ & $1.12 \mathrm{E}+09$ & $8.17 \mathrm{E}+08$ & $7.78 \mathrm{E}+08$ & $7.39 \mathrm{E}+08$ & $7.07 \mathrm{E}+08$ & $6.83 \mathrm{E}+08$ & $6.64 \mathrm{E}+08$ & $6.48 E+08$ & $6.35 \mathrm{E}+08$ \\
\hline $1.50 \mathrm{E}-01$ & $3.00 \mathrm{E}-01$ & $1.34 \mathrm{E}+09$ & $7.73 E+08$ & $6.82 \mathrm{E}+08$ & $6.92 \mathrm{E}+08$ & $6.89 E+08$ & $6.84 \mathrm{E}+08$ & $6.79 E+08$ & $6.74 \mathrm{E}+08$ & $6.68 \mathrm{E}+08$ & $6.63 E+08$ \\
\hline $3.00 \mathrm{E}-01$ & $4.00 \mathrm{E}-01$ & $4.99 \mathrm{E}+08$ & $2.59 \mathrm{E}+08$ & $2.19 \mathrm{E}+08$ & $2.25 \mathrm{E}+08$ & $2.26 \mathrm{E}+08$ & $2.26 \mathrm{E}+08$ & $2.26 \mathrm{E}+08$ & $2.25 \mathrm{E}+08$ & $2.24 \mathrm{E}+08$ & $2.23 E+08$ \\
\hline $4.00 \mathrm{E}-01$ & $5.10 \mathrm{E}-01$ & $5.26 \mathrm{E}+08$ & $3.10 \mathrm{E}+08$ & $1.68 \mathrm{E}+08$ & $1.43 \mathrm{E}+08$ & $1.36 \mathrm{E}+08$ & $1.33 \mathrm{E}+08$ & $1.32 \mathrm{E}+08$ & $1.31 \mathrm{E}+08$ & $1.30 \mathrm{E}+08$ & $1.29 \mathrm{E}+08$ \\
\hline $5.10 \mathrm{E}-01$ & $6.00 \mathrm{E}-01$ & $8.70 E+08$ & $1.27 \mathrm{E}+09$ & $3.95 \mathrm{E}+08$ & $1.28 \mathrm{E}+08$ & $6.41 E+07$ & $4.69 \mathrm{E}+07$ & $4.06 \mathrm{E}+07$ & $3.72 E+07$ & $3.48 \mathrm{E}+07$ & $3.30 \mathrm{E}+07$ \\
\hline $6.00 \mathrm{E}-01$ & $7.00 \mathrm{E}-01$ & $3.93 \mathrm{E}+09$ & $9.87 \mathrm{E}+09$ & $8.59 \mathrm{E}+09$ & $8.03 E+09$ & $7.95 \mathrm{E}+09$ & $7.97 \mathrm{E}+09$ & $8.02 \mathrm{E}+09$ & $8.06 \mathrm{E}+09$ & $8.08 \mathrm{E}+09$ & $8.10 \mathrm{E}+09$ \\
\hline $7.00 \mathrm{E}-01$ & $1.00 \mathrm{E}+00$ & $6.12 \mathrm{E}+09$ & $4.52 \mathrm{E}+09$ & $1.49 \mathrm{E}+09$ & $5.09 E+08$ & $2.56 \mathrm{E}+08$ & $1.77 \mathrm{E}+08$ & $1.40 \mathrm{E}+08$ & $1.18 \mathrm{E}+08$ & $1.01 E+08$ & $8.91 E+07$ \\
\hline $1.00 \mathrm{E}+00$ & $1.50 \mathrm{E}+00$ & $3.28 \mathrm{E}+08$ & $5.43 \mathrm{E}+08$ & $3.28 \mathrm{E}+08$ & $2.18 \mathrm{E}+08$ & $1.61 \mathrm{E}+08$ & $1.24 \mathrm{E}+08$ & $9.80 \mathrm{E}+07$ & $7.83 E+07$ & $6.33 E+07$ & $5.20 \mathrm{E}+07$ \\
\hline $1.50 \mathrm{E}+00$ & $2.00 \mathrm{E}+00$ & $3.30 \mathrm{E}+07$ & $1.63 \mathrm{E}+07$ & $1.04 \mathrm{E}+07$ & $8.45 \mathrm{E}+06$ & $6.83 \mathrm{E}+06$ & $5.55 \mathrm{E}+06$ & $4.58 \mathrm{E}+06$ & $3.84 \mathrm{E}+06$ & $3.27 \mathrm{E}+06$ & $2.84 \mathrm{E}+06$ \\
\hline $2.00 \mathrm{E}+00$ & $2.50 \mathrm{E}+00$ & $8.62 E+07$ & $1.61 \mathrm{E}+07$ & $3.39 \mathrm{E}+05$ & $3.43 \mathrm{E}+04$ & $2.95 \mathrm{E}+04$ & $2.94 \mathrm{E}+04$ & $2.94 \mathrm{E}+04$ & $2.94 \mathrm{E}+04$ & $2.92 \mathrm{E}+04$ & $2.91 \mathrm{E}+04$ \\
\hline $2.50 \mathrm{E}+00$ & $3.00 \mathrm{E}+00$ & $2.75 \mathrm{E}+05$ & $9.98 \mathrm{E}+04$ & $1.08 \mathrm{E}+04$ & $8.68 \mathrm{E}+03$ & $9.81 \mathrm{E}+03$ & $1.08 \mathrm{E}+04$ & $1.17 \mathrm{E}+04$ & $1.25 \mathrm{E}+04$ & $1.34 \mathrm{E}+04$ & $1.44 \mathrm{E}+04$ \\
\hline $3.00 \mathrm{E}+00$ & $4.00 \mathrm{E}+00$ & $2.54 \mathrm{E}+04$ & $1.06 \mathrm{E}+04$ & $5.43 E+02$ & $2.69 \mathrm{E}+01$ & $6.38 \mathrm{E}+00$ & $5.36 \mathrm{E}+00$ & $5.11 \mathrm{E}+00$ & $4.90 \mathrm{E}+00$ & $4.71 \mathrm{E}+00$ & $4.55 \mathrm{E}+00$ \\
\hline $4.00 \mathrm{E}+00$ & $5.00 \mathrm{E}+00$ & $2.99 \mathrm{E}-01$ & $1.58 \mathrm{E}+00$ & $1.97 \mathrm{E}+00$ & $1.95 \mathrm{E}+00$ & $1.87 \mathrm{E}+00$ & $1.79 \mathrm{E}+00$ & $1.71 \mathrm{E}+00$ & $1.64 \mathrm{E}+00$ & $1.57 \mathrm{E}+00$ & $1.52 \mathrm{E}+00$ \\
\hline $5.00 \mathrm{E}+00$ & $6.00 \mathrm{E}+00$ & $1.01 \mathrm{E}-01$ & $5.30 \mathrm{E}-01$ & $6.63 \mathrm{E}-01$ & $6.55 \mathrm{E}-01$ & $6.28 \mathrm{E}-01$ & $5.99 \mathrm{E}-01$ & $5.73 \mathrm{E}-01$ & $5.49 \mathrm{E}-01$ & $5.26 \mathrm{E}-01$ & $5.07 \mathrm{E}-01$ \\
\hline $6.00 \mathrm{E}+00$ & $7.00 \mathrm{E}+00$ & $3.38 \mathrm{E}-02$ & $1.78 \mathrm{E}-01$ & $2.23 \mathrm{E}-01$ & $2.20 \mathrm{E}-01$ & $2.11 \mathrm{E}-01$ & $2.01 \mathrm{E}-01$ & $1.92 \mathrm{E}-01$ & $1.84 \mathrm{E}-01$ & $1.76 \mathrm{E}-01$ & $1.70 \mathrm{E}-01$ \\
\hline $7.00 \mathrm{E}+00$ & $7.50 \mathrm{E}+00$ & $7.26 \mathrm{E}-03$ & $3.82 \mathrm{E}-02$ & $4.78 \mathrm{E}-02$ & $4.72 \mathrm{E}-02$ & $4.52 \mathrm{E}-02$ & $4.31 \mathrm{E}-02$ & $4.12 \mathrm{E}-02$ & $3.94 \mathrm{E}-02$ & $3.77 \mathrm{E}-02$ & 3.63E-02 \\
\hline $7.50 \mathrm{E}+00$ & $8.00 \mathrm{E}+00$ & $4.21 \mathrm{E}-03$ & $2.21 \mathrm{E}-02$ & $2.77 \mathrm{E}-02$ & $2.74 \mathrm{E}-02$ & $2.62 \mathrm{E}-02$ & $2.50 \mathrm{E}-02$ & $2.38 \mathrm{E}-02$ & $2.28 \mathrm{E}-02$ & $2.18 \mathrm{E}-02$ & $2.10 \mathrm{E}-02$ \\
\hline $8.00 \mathrm{E}+00$ & $1.00 \mathrm{E}+01$ & $4.96 \mathrm{E}-03$ & $2.61 \mathrm{E}-02$ & $3.27 \mathrm{E}-02$ & $3.23 \mathrm{E}-02$ & $3.09 \mathrm{E}-02$ & $2.94 \mathrm{E}-02$ & $2.81 \mathrm{E}-02$ & $2.69 \mathrm{E}-02$ & $2.57 \mathrm{E}-02$ & $2.47 \mathrm{E}-02$ \\
\hline $1.00 \mathrm{E}+01$ & $1.40 \mathrm{E}+01$ & $2.35 \mathrm{E}-04$ & $1.24 \mathrm{E}-03$ & $1.55 \mathrm{E}-03$ & $1.53 \mathrm{E}-03$ & $1.46 \mathrm{E}-03$ & $1.39 \mathrm{E}-03$ & $1.33 \mathrm{E}-03$ & $1.27 \mathrm{E}-03$ & $1.21 \mathrm{E}-03$ & $1.17 \mathrm{E}-03$ \\
\hline Totals & & $2.88 \mathrm{E}+10$ & $2.69 \mathrm{E}+10$ & $2.01 E+10$ & $1.83 \mathrm{E}+10$ & $1.77 \mathrm{E}+10$ & $1.76 \mathrm{E}+10$ & $1.75 \mathrm{E}+10$ & $1.74 \mathrm{E}+10$ & $1.73 \mathrm{E}+10$ & $1.73 E+10$ \\
\hline
\end{tabular}


Table A.5 Gamma Source Spectra (gammas/s.Ci),VELM 23 Grp. HFED U6Fe, Module 23, Slot 6, 19.84\% Enrichment, 27\% Depleted, 5.59E-02 MW/gU

\begin{tabular}{|c|c|c|c|c|c|c|c|c|c|c|c|}
\hline \multicolumn{12}{|c|}{ Decay Period } \\
\hline Energy & interval & $1 Y$ & $5 Y$ & $10 \mathrm{Y}$ & $15 Y$ & $20 Y$ & $25 Y$ & $30 Y$ & $35 Y$ & $40 Y$ & $45 Y$ \\
\hline \multicolumn{2}{|c|}{$(\mathrm{MeV})$} & & & & & & & & & & \\
\hline & & & & & & & & & & & \\
\hline $1.00 \mathrm{E}-02$ & $2.00 \mathrm{E}-02$ & $3.78 \mathrm{E}+09$ & $2.55 \mathrm{E}+09$ & $2.38 \mathrm{E}+09$ & $2.48 \mathrm{E}+09$ & $2.51 \mathrm{E}+09$ & $2.53 \mathrm{E}+09$ & $2.52 \mathrm{E}+09$ & $2.53 \mathrm{E}+09$ & $2.52 \mathrm{E}+09$ & $2.52 \mathrm{E}+09$ \\
\hline $2.00 \mathrm{E}-02$ & $4.50 \mathrm{E}-02$ & $4.92 E+09$ & $3.16 \mathrm{E}+09$ & $2.82 \mathrm{E}+09$ & $2.92 \mathrm{E}+09$ & $2.95 \mathrm{E}+09$ & $2.96 \mathrm{E}+09$ & $2.96 \mathrm{E}+09$ & $2.96 \mathrm{E}+09$ & $2.95 \mathrm{E}+09$ & $2.95 \mathrm{E}+09$ \\
\hline $4.50 \mathrm{E}-02$ & $7.00 \mathrm{E}-02$ & $1.82 \mathrm{E}+09$ & $1.18 \mathrm{E}+09$ & $1.08 \mathrm{E}+09$ & $1.13 \mathrm{E}+09$ & $1.15 \mathrm{E}+09$ & $1.15 \mathrm{E}+09$ & $1.15 \mathrm{E}+09$ & $1.16 \mathrm{E}+09$ & $1.16 \mathrm{E}+09$ & $1.16 \mathrm{E}+09$ \\
\hline $7.00 \mathrm{E}-02$ & $1.00 \mathrm{E}-01$ & $1.51 \mathrm{E}+09$ & $8.75 \mathrm{E}+08$ & $7.40 \mathrm{E}+08$ & $7.68 \mathrm{E}+08$ & $7.74 E+08$ & $7.77 \mathrm{E}+08$ & $7.74 \mathrm{E}+08$ & $7.74 \mathrm{E}+08$ & $7.70 \mathrm{E}+08$ & $7.69 \mathrm{E}+08$ \\
\hline $1.00 \mathrm{E}-01$ & $1.50 \mathrm{E}-01$ & $2.31 \mathrm{E}+09$ & $9.75 E+08$ & $6.36 \mathrm{E}+08$ & $6.48 \mathrm{E}+08$ & $6.47 \mathrm{E}+08$ & $6.43 \mathrm{E}+08$ & $6.37 \mathrm{E}+08$ & $6.34 \mathrm{E}+08$ & $6.28 \mathrm{E}+08$ & $6.25 \mathrm{E}+08$ \\
\hline $1.50 \mathrm{E}-01$ & $3.00 \mathrm{E}-01$ & $1.28 \mathrm{E}+09$ & $7.49 \mathrm{E}+08$ & $6.39 \mathrm{E}+08$ & $6.67 E+08$ & $6.75 E+08$ & $6.78 \mathrm{E}+08$ & $6.76 \mathrm{E}+08$ & $6.76 \mathrm{E}+08$ & $6.73 E+08$ & $6.71 E+08$ \\
\hline $3.00 \mathrm{E}-01$ & $4.00 \mathrm{E}-01$ & $4.80 \mathrm{E}+08$ & $2.64 \mathrm{E}+08$ & $2.14 \mathrm{E}+08$ & $2.23 \mathrm{E}+08$ & $2.27 E+08$ & $2.28 \mathrm{E}+08$ & $2.28 \mathrm{E}+08$ & $2.28 \mathrm{E}+08$ & $2.27 \mathrm{E}+08$ & $2.26 \mathrm{E}+08$ \\
\hline $4.00 \mathrm{E}-01$ & $5.10 \mathrm{E}-01$ & $5.23 \mathrm{E}+08$ & $2.63 E+08$ & $1.47 \mathrm{E}+08$ & $1.36 \mathrm{E}+08$ & $1.34 \mathrm{E}+08$ & $1.33 \mathrm{E}+08$ & $1.32 \mathrm{E}+08$ & $1.32 \mathrm{E}+08$ & $1.31 \mathrm{E}+08$ & $1.31 E+08$ \\
\hline $5.10 \mathrm{E}-01$ & $6.00 \mathrm{E}-01$ & $2.35 \mathrm{E}+08$ & $2.42 \mathrm{E}+08$ & $8.42 \mathrm{E}+07$ & $4.62 \mathrm{E}+07$ & $3.67 \mathrm{E}+07$ & $3.37 \mathrm{E}+07$ & $3.22 \mathrm{E}+07$ & $3.13 E+07$ & $3.06 \mathrm{E}+07$ & $3.01 \mathrm{E}+07$ \\
\hline $6.00 \mathrm{E}-01$ & $7.00 \mathrm{E}-01$ & $1.36 \mathrm{E}+09$ & $5.84 \mathrm{E}+09$ & $7.46 \mathrm{E}+09$ & $7.86 E+09$ & $8.02 E+09$ & $8.13 E+09$ & $8.18 \mathrm{E}+09$ & $8.25 E+09$ & $8.28 \mathrm{E}+09$ & $8.33 E+09$ \\
\hline $7.00 \mathrm{E}-01$ & $1.00 \mathrm{E}+00$ & $5.65 \mathrm{E}+09$ & $6.41 E+08$ & $2.55 E+08$ & $1.29 \mathrm{E}+08$ & $9.39 \mathrm{E}+07$ & $8.09 \mathrm{E}+07$ & $7.36 \mathrm{E}+07$ & $6.89 \mathrm{E}+07$ & $6.51 \mathrm{E}+07$ & $6.24 \mathrm{E}+07$ \\
\hline $1.00 \mathrm{E}+00$ & $1.50 \mathrm{E}+00$ & $1.32 \mathrm{E}+08$ & $1.23 \mathrm{E}+08$ & $7.77 \mathrm{E}+07$ & $6.03 E+07$ & $4.96 \mathrm{E}+07$ & $4.20 \mathrm{E}+07$ & $3.61 \mathrm{E}+07$ & $3.19 \mathrm{E}+07$ & $2.85 \mathrm{E}+07$ & $2.60 \mathrm{E}+07$ \\
\hline $1.50 \mathrm{E}+00$ & $2.00 \mathrm{E}+00$ & $2.98 \mathrm{E}+07$ & $1.07 \mathrm{E}+07$ & $3.56 \mathrm{E}+06$ & $3.07 \mathrm{E}+06$ & $2.74 \mathrm{E}+06$ & $2.48 \mathrm{E}+06$ & $2.26 \mathrm{E}+06$ & $2.10 \mathrm{E}+06$ & $1.97 \mathrm{E}+06$ & $1.87 \mathrm{E}+06$ \\
\hline $2.00 \mathrm{E}+00$ & $2.50 \mathrm{E}+00$ & $8.22 E+07$ & $1.89 \mathrm{E}+07$ & $4.08 \mathrm{E}+05$ & $3.57 \mathrm{E}+04$ & $2.97 \mathrm{E}+04$ & $2.98 \mathrm{E}+04$ & $2.98 \mathrm{E}+04$ & $2.98 \mathrm{E}+04$ & $2.97 \mathrm{E}+04$ & $2.96 \mathrm{E}+04$ \\
\hline $2.50 \mathrm{E}+00$ & $3.00 \mathrm{E}+00$ & $2.79 \mathrm{E}+05$ & $1.23 \mathrm{E}+05$ & $6.00 \mathrm{E}+03$ & $3.75 \mathrm{E}+02$ & $1.80 \mathrm{E}+02$ & $1.90 \mathrm{E}+02$ & $2.05 \mathrm{E}+02$ & $2.21 E+02$ & $2.37 \mathrm{E}+02$ & $2.55 \mathrm{E}+02$ \\
\hline $3.00 \mathrm{E}+00$ & $4.00 \mathrm{E}+00$ & $2.64 \mathrm{E}+04$ & $1.35 \mathrm{E}+04$ & $7.11 \mathrm{E}+02$ & $2.85 \mathrm{E}+01$ & $1.28 \mathrm{E}+00$ & $2.60 \mathrm{E}-01$ & $2.42 \mathrm{E}-01$ & $2.67 \mathrm{E}-01$ & $2.94 \mathrm{E}-01$ & $3.26 \mathrm{E}-01$ \\
\hline $4.00 \mathrm{E}+00$ & $5.00 \mathrm{E}+00$ & $5.66 \mathrm{E}-03$ & $3.40 \mathrm{E}-02$ & $5.13 \mathrm{E}-02$ & $5.93 \mathrm{E}-02$ & $6.58 \mathrm{E}-02$ & $7.26 \mathrm{E}-02$ & $7.98 \mathrm{E}-02$ & $8.83 \mathrm{E}-02$ & $9.74 \mathrm{E}-02$ & $1.08 \mathrm{E}-01$ \\
\hline $5.00 \mathrm{E}+00$ & $6.00 \mathrm{E}+00$ & $1.90 \mathrm{E}-03$ & $1.14 \mathrm{E}-02$ & $1.71 \mathrm{E}-02$ & $1.98 \mathrm{E}-02$ & $2.19 \mathrm{E}-02$ & $2.41 \mathrm{E}-02$ & $2.65 \mathrm{E}-02$ & $2.93 \mathrm{E}-02$ & $3.23 \mathrm{E}-02$ & $3.58 \mathrm{E}-02$ \\
\hline $6.00 \mathrm{E}+00$ & $7.00 \mathrm{E}+00$ & $6.36 \mathrm{E}-04$ & $3.80 \mathrm{E}-03$ & $5.72 \mathrm{E}-03$ & $6.60 \mathrm{E}-03$ & $7.30 \mathrm{E}-03$ & $8.05 \mathrm{E}-03$ & $8.84 \mathrm{E}-03$ & $9.76 \mathrm{E}-03$ & $1.08 \mathrm{E}-02$ & $1.19 \mathrm{E}-02$ \\
\hline $7.00 \mathrm{E}+00$ & $7.50 \mathrm{E}+00$ & $1.36 \mathrm{E}-04$ & $8.14 \mathrm{E}-04$ & $1.22 \mathrm{E}-03$ & $1.41 \mathrm{E}-03$ & $1.56 \mathrm{E}-03$ & $1.72 \mathrm{E}-03$ & $1.89 \mathrm{E}-03$ & $2.08 \mathrm{E}-03$ & $2.30 \mathrm{E}-03$ & $2.55 \mathrm{E}-03$ \\
\hline $7.50 \mathrm{E}+00$ & $8.00 \mathrm{E}+00$ & $7.89 \mathrm{E}-05$ & $4.71 \mathrm{E}-04$ & $7.08 \mathrm{E}-04$ & $8.15 \mathrm{E}-04$ & $9.02 \mathrm{E}-04$ & $9.94 \mathrm{E}-04$ & $1.09 \mathrm{E}-03$ & $1.20 \mathrm{E}-03$ & $1.33 \mathrm{E}-03$ & $1.47 \mathrm{E}-03$ \\
\hline $8.00 \mathrm{E}+\infty 0$ & $1.00 \mathrm{E}+01$ & $9.29 \mathrm{E}-05$ & $5.54 \mathrm{E}-04$ & $8.33 \mathrm{E}-04$ & $9.59 \mathrm{E}-04$ & $1.06 \mathrm{E}-03$ & $1.17 \mathrm{E}-03$ & $1.28 \mathrm{E}-03$ & $1.41 \mathrm{E}-03$ & $1.56 \mathrm{E}-03$ & $1.73 \mathrm{E}-03$ \\
\hline $1.00 \mathrm{E}+01$ & $1.40 \mathrm{E}+01$ & $4.39 \mathrm{E}-06$ & $2.62 \mathrm{E}-05$ & $3.93 \mathrm{E}-05$ & $4.52 \mathrm{E}-05$ & $5.00 \mathrm{E}-05$ & $5.50 \mathrm{E}-05$ & $6.03 \mathrm{E}-05$ & $6.66 \mathrm{E}-05$ & $7.34 \mathrm{E}-05$ & $8.13 \mathrm{E}-05$ \\
\hline Totals & & $2.41 E+10$ & $1.69 \mathrm{E}+10$ & $1.65 \mathrm{E}+10$ & $1.71 \mathrm{E}+10$ & $1.73 E+10$ & $1.74 E+10$ & $1.74 \mathrm{E}+10$ & $1.75 \mathrm{E}+10$ & $1.75 \mathrm{E}+10$ & $1.75 E+10$ \\
\hline
\end{tabular}





\section{APPENDIX B}

Major Isotopic Activity (Ci) for the MFPs from the $50-\mathrm{MWd} / \mathrm{kgU}, 40-\mathrm{kW} / \mathrm{kgU}$ Case 
.

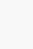


Table B.1 Light Element Activity at 15-Year Decay Period $50 \mathrm{MWd} / \mathrm{kgU}, 40 \mathrm{~kW} / \mathrm{kgU}, \mathrm{U}-\mathrm{wt} \%$ (U234 =.037, U235=4.200, $\mathrm{U} 236=.019$, U238 $=95.744$ ); Continuous Operation $=1250.00 \mathrm{~d}$;

Tbl A.4 ORNL-6698. Cycle 3 of 5. Clad is Zircaloy: Wt\% (97.91 Zr, 1.59 Sn, 0.5 Fe). SCALE 4.2 Composition Library. See Tbl. 3.11, ORNL-6698 for composition.

\begin{tabular}{|l|c|c|c|}
\hline \multicolumn{5}{|c|}{ Light Elements (Ci/Mg) } \\
\hline \multicolumn{1}{|c|}{ Isotope } & 15 Yr & \% Total & Running $\%$ \\
\hline & & & \\
\hline Sb-125 & $2.85 \mathrm{E}+01$ & $5.43 \mathrm{E}+01$ & $5.43 \mathrm{E}+01$ \\
\hline Fe-55 & $1.51 \mathrm{E}+01$ & $2.88 \mathrm{E}+01$ & $8.30 \mathrm{E}+01$ \\
\hline Te-125m & $6.95 \mathrm{E}+01$ & $1.32 \mathrm{E}+01$ & $9.63 \mathrm{E}+01$ \\
\hline Sn-121m & $8.53 \mathrm{E}+01$ & $1.62 \mathrm{E}+01$ & $9.79 \mathrm{E}+01$ \\
\hline $\mathrm{Sn}-121$ & $6.62 \mathrm{E}-01$ & $1.26 \mathrm{E}+00$ & $9.92 \mathrm{E}+01$ \\
\hline $\mathrm{Co}-60$ & $1.80 \mathrm{E}-01$ & $3.43 \mathrm{E}-01$ & $9.95 \mathrm{E}+01$ \\
\hline $\mathrm{Zr}-93$ & $1.79 \mathrm{E}-01$ & $3.41 \mathrm{E}-01$ & $9.99 \mathrm{E}+01$ \\
\hline $\mathrm{Nb}-93 \mathrm{~m}$ & $9.13 \mathrm{E}-02$ & $1.74 \mathrm{E}-01$ & $1.00 \mathrm{E}+02$ \\
\hline $\mathrm{Sn}-119 \mathrm{~m}$ & $2.42 \mathrm{E}-02$ & $4.61 \mathrm{E}-02$ & $1.00 \mathrm{E}+02$ \\
\hline $\mathrm{Sr}-90$ & $2.61 \mathrm{E}-03$ & $4.97 \mathrm{E}-03$ & $1.00 \mathrm{E}+02$ \\
\hline $\mathrm{Y}-90$ & $2.61 \mathrm{E}-03$ & $4.97 \mathrm{E}-03$ & $1.00 \mathrm{E}+02$ \\
\hline $\mathrm{Mn}-54$ & $8.46 \mathrm{E}-04$ & $1.61 \mathrm{E}-03$ & $1.00 \mathrm{E}+02$ \\
\hline $\mathrm{Cd}-113 \mathrm{~m}$ & $1.85 \mathrm{E}-05$ & $3.52 \mathrm{E}-05$ & $1.00 \mathrm{E}+02$ \\
\hline Tc-99 & $3.42 \mathrm{E}-06$ & $6.51 \mathrm{E}-06$ & $1.00 \mathrm{E}+02$ \\
\hline $\mathrm{H}-3$ & $9.15 \mathrm{E}-08$ & $1.74 \mathrm{E}-07$ & $1.00 \mathrm{E}+02$ \\
\hline $\mathrm{Ni}-63$ & $3.49 \mathrm{E}-09$ & $6.65 \mathrm{E}-09$ & $1.00 \mathrm{E}+02$ \\
\hline $\mathrm{Nb}-94$ & $1.85 \mathrm{E}-09$ & $3.52 \mathrm{E}-09$ & $1.00 \mathrm{E}+02$ \\
\hline $\mathrm{Sn}-123$ & $6.25 \mathrm{E}-11$ & $1.19 \mathrm{E}-10$ & $1.00 \mathrm{E}+02$ \\
\hline $\mathrm{In}-113 \mathrm{~m}$ & $1.11 \mathrm{E}-11$ & $2.11 \mathrm{E}-11$ & $1.00 \mathrm{E}+02$ \\
\hline $\mathrm{Sn}-113$ & $1.10 \mathrm{E}-11$ & $2.10 \mathrm{E}-11$ & $1.00 \mathrm{E}+02$ \\
\hline Te-123m & $1.67 \mathrm{E}-13$ & $3.18 \mathrm{E}-13$ & $1.00 \mathrm{E}+02$ \\
\hline Te-127m & $3.94 \mathrm{E}-17$ & $7.50 \mathrm{E}-17$ & $1.00 \mathrm{E}+02$ \\
\hline Te-127 & $3.86 \mathrm{E}-17$ & $7.35 \mathrm{E}-17$ & $1.00 \mathrm{E}+02$ \\
\hline $\mathrm{Nb}-95$ & $2.04 \mathrm{E}-21$ & $3.89 \mathrm{E}-21$ & $1.00 \mathrm{E}+02$ \\
\hline $\mathrm{Zr}-95$ & $9.27 \mathrm{E}-22$ & $1.77 \mathrm{E}-21$ & $1.00 \mathrm{E}+02$ \\
\hline $\mathrm{Nb}-95 \mathrm{~m}$ & $1.09 \mathrm{E}-23$ & $2.08 \mathrm{E}-23$ & $1.00 \mathrm{E}+02$ \\
\hline Sb-124 & $1.57 \mathrm{E}-26$ & $2.99 \mathrm{E}-26$ & $1.00 \mathrm{E}+02$ \\
\hline $\mathrm{Y}-91$ & $1.23 \mathrm{E}-26$ & $2.34 \mathrm{E}-26$ & $1.00 \mathrm{E}+02$ \\
\hline $\mathrm{Co}-58$ & $2.82 \mathrm{E}-28$ & $5.37 \mathrm{E}-28$ & $1.00 \mathrm{E}+02$ \\
\hline $\mathrm{Dr}-89$ & $1.79 \mathrm{E}-31$ & $3.41 \mathrm{E}-31$ & $1.00 \mathrm{E}+02$ \\
\hline In-114m & $1.58 \mathrm{E}-31$ & $3.01 \mathrm{E}-31$ & $1.00 \mathrm{E}+02$ \\
\hline & & & \\
\hline Totals & $5.25 \mathrm{E}+01$ & $1.00 \mathrm{E}+02$ & \\
\hline
\end{tabular}


Table B.2 Actinide Activity at 15-Year Decay Period

$50 \mathrm{MWd} / \mathrm{kgU}, 40 \mathrm{~kW} / \mathrm{kgU}, \mathrm{U}-\mathrm{wt} \%(\mathrm{U} 234=.037$,

$\mathrm{U} 235=4.200, \mathrm{U} 236=.019, \mathrm{U} 238=95.744)$; Continuous

Operation $=1250.00 \mathrm{~d}$; Tbl A.4 ORNL-6698. Cycle 3 of 5 . Clad is Zircaloy:

Wt\%(97.91 Zr, $1.59 \mathrm{Sn}, 0.5 \mathrm{Fe}$ ). SCALE 4.2 Composition Library.

See Tbl 3.11, ORNL-6698 for composition.

\begin{tabular}{|c|c|c|c|}
\hline \multicolumn{4}{|c|}{ Actinides $(\mathrm{Ci} / \mathrm{Mg})$} \\
\hline Element & $15 Y_{T}$ & $\%$ Total & Running \% \\
\hline $\mathrm{Pu}-241$ & $1.08 \mathrm{E}+05$ & $8.78 \mathrm{E}+01$ & $8.78 \mathrm{E}+01$ \\
\hline $\mathrm{Pu}-238$ & $6.00 \mathrm{E}+03$ & $4.88 \mathrm{E}+00$ & $9.27 \mathrm{E}+01$ \\
\hline $\mathrm{Cm}-244$ & $4.19 E+03$ & $3.41 \mathrm{E}+00$ & $9.61 E+01$ \\
\hline Am-241 & $3.97 \mathrm{E}+03$ & $3.23 E+00$ & $9.93 E+01$ \\
\hline $\mathrm{Pu}-240$ & $5.25 \mathrm{E}+02$ & $4.27 \mathrm{E}-01$ & $9.97 \mathrm{E}+01$ \\
\hline Pu-239 & $3.99 E+02$ & $3.24 \mathrm{E}-01$ & $1.00 \mathrm{E}+02$ \\
\hline Np-239 & $5.09 \mathrm{E}+01$ & $4.14 \mathrm{E}-02$ & $1.00 \mathrm{E}+02$ \\
\hline $\mathrm{Am}-243$ & $5.09 \mathrm{E}+01$ & $4.14 \mathrm{E}-02$ & $1.00 E+02$ \\
\hline $\mathrm{Cm}-243$ & $3.74 \mathrm{E}+01$ & $3.04 \mathrm{E}-02$ & $1.00 \mathrm{E}+02$ \\
\hline Am-242m & $1.88 \mathrm{E}+01$ & $1.53 \mathrm{E}-02$ & $1.00 \mathrm{E}+02$ \\
\hline Am-242 & $1.87 \mathrm{E}+01$ & $1.52 \mathrm{E}-02$ & $1.00 \mathrm{E}+02$ \\
\hline $\mathrm{Cm}-242$ & $1.55 \mathrm{E}+01$ & $1.26 \mathrm{E}-02$ & $1.00 \mathrm{E}+02$ \\
\hline $\mathrm{Pu}-242$ & $3.36 \mathrm{E}+00$ & $2.73 E-03$ & $1.00 \mathrm{E}+02$ \\
\hline $\mathrm{U}-237$ & $2.58 E+00$ & $2.10 \mathrm{E}-03$ & $1.00 E+02$ \\
\hline $\mathrm{U}-234$ & $1.39 E+00$ & $1.13 \mathrm{E}-03$ & $1.00 \mathrm{E}+02$ \\
\hline $\mathrm{Cm}-245$ & $7.99 \mathrm{E}-01$ & $6.50 \mathrm{E}-04$ & $1.00 \mathrm{E}+02$ \\
\hline $\mathrm{Pa}-233$ & $6.13 E-01$ & $4.98 \mathrm{E}-04$ & $1.00 \mathrm{E}+02$ \\
\hline $\mathrm{Np}-237$ & $6.13 E-01$ & $4.98 \mathrm{E}-04$ & $1.00 \mathrm{E}+02$ \\
\hline $\mathrm{U}-236$ & $3.77 \mathrm{E}-01$ & 3.07E-04 & $1.00 \mathrm{E}+02$ \\
\hline Th-234 & $3.10 \mathrm{E}-01$ & $2.52 \mathrm{E}-04$ & $1.00 \mathrm{E}+02$ \\
\hline $\mathrm{Pa}-234 \mathrm{~m}$ & $3.10 \mathrm{E}-01$ & $2.52 \mathrm{E}-04$ & $1.00 \mathrm{E}+02$ \\
\hline U-238 & $3.10 \mathrm{E}-01$ & $2.52 \mathrm{E}-04$ & $1.00 \mathrm{E}+02$ \\
\hline $\mathrm{Cm}-246$ & $2.31 \mathrm{E}-01$ & $1.88 \mathrm{E}-04$ & $1.00 \mathrm{E}+02$ \\
\hline $\mathrm{U}-232$ & $1.57 \mathrm{E}-01$ & $1.28 \mathrm{E}-04$ & $1.00 \mathrm{E}+02$ \\
\hline $\mathrm{Pb}-212$ & $1.54 \mathrm{E}-01$ & $1.25 \mathrm{E}-04$ & $1.00 \mathrm{E}+02$ \\
\hline $\mathrm{Bi}-212$ & $1.54 \mathrm{E}-01$ & $1.25 \mathrm{E}-04$ & $1.00 \mathrm{E}+02$ \\
\hline Po-216 & $1.54 \mathrm{E}-01$ & $1.25 E-04$ & $1.00 \mathrm{E}+02$ \\
\hline $\mathrm{U}-235$ & $1.63 \mathrm{E}-02$ & $1.33 \mathrm{E}-05$ & $1.00 \mathrm{E}+02$ \\
\hline $\mathrm{Pa}-234$ & $4.03 \mathrm{E}-04$ & $3.28 \mathrm{E}-07$ & $1.00 E+02$ \\
\hline Cf-250 & $2.94 \mathrm{E}-04$ & 2.39E-07 & $1.00 \mathrm{E}+02$ \\
\hline Totals & $1.23 E+05$ & & \\
\hline
\end{tabular}

B-4 
Table B.3 Fission Product Activity at 15-Year Decay Period $50 \mathrm{MWd} / \mathrm{kgU}, 40 \mathrm{~kW} / \mathrm{kgU}$, U-wt\% (U234 $=.037$,

$\mathrm{U} 235=4.200, \mathrm{U} 236=.019$, U238 $=95.744)$; Continuous Operation $=1250.00 \mathrm{~d} ;$ Tbl A.4 ORNL-6698. Cycle 3 of 5 . Clad is Zircaloy: Wt\%( $97.91 \mathrm{Zr}, 1.59 \mathrm{Sn}, 0.5 \mathrm{Fe})$. SCALE 4.2 Composition Library. See Tbl 3.11, ORNL-6698 for composition.

\begin{tabular}{|l|c|c|c|}
\hline \multicolumn{4}{|c|}{ Fission Products (Ci/Mg) } \\
\hline \multicolumn{1}{|c|}{ Element } & 15 Yr & \% Total & Running \% \\
\hline & & & \\
\hline Cs137 & $1.15 \mathrm{E}+05$ & $2.90 \mathrm{E}+01$ & $2.90 \mathrm{E}+01$ \\
\hline Ba137m & $1.08 \mathrm{E}+05$ & $2.72 \mathrm{E}+01$ & $5.62 \mathrm{E}+01$ \\
\hline Sr 90 & $7.72 \mathrm{E}+04$ & $1.94 \mathrm{E}+01$ & $7.56 \mathrm{E}+01$ \\
\hline Y 90 & $7.72 \mathrm{E}+04$ & $1.94 \mathrm{E}+01$ & $9.51 \mathrm{E}+01$ \\
\hline Eu154 & $5.92 \mathrm{E}+03$ & $1.49 \mathrm{E}+00$ & $9.66 \mathrm{E}+01$ \\
\hline Kr 85 & $4.89 \mathrm{E}+03$ & $1.23 \mathrm{E}+00$ & $9.78 \mathrm{E}+01$ \\
\hline Pm147 & $3.66 \mathrm{E}+03$ & $9.22 \mathrm{E}-01$ & $9.88 \mathrm{E}+01$ \\
\hline Cs134 & $1.89 \mathrm{E}+03$ & $4.76 \mathrm{E}-01$ & $9.93 \mathrm{E}+01$ \\
\hline Eu155 & $1.60 \mathrm{E}+03$ & $4.03 \mathrm{E}-01$ & $9.97 \mathrm{E}+01$ \\
\hline Sm151 & $5.48 \mathrm{E}+02$ & $1.38 \mathrm{E}-01$ & $9.98 \mathrm{E}+01$ \\
\hline H 3 & $3.11 \mathrm{E}+02$ & $7.83 \mathrm{E}-02$ & $9.99 \mathrm{E}+01$ \\
\hline Sb125 & $2.56 \mathrm{E}+02$ & $6.45 \mathrm{E}-02$ & $1.00 \mathrm{E}+02$ \\
\hline Te125m & $6.26 \mathrm{E}+01$ & $1.58 \mathrm{E}-02$ & $1.00 \mathrm{E}+02$ \\
\hline Ru106 & $2.90 \mathrm{E}+01$ & $7.30 \mathrm{E}-03$ & $1.00 \mathrm{E}+02$ \\
\hline Rh106 & $2.90 \mathrm{E}+01$ & $7.30 \mathrm{E}-03$ & $1.00 \mathrm{E}+02$ \\
\hline Cd113m & $2.37 \mathrm{E}+01$ & $5.97 \mathrm{E}-03$ & $1.00 \mathrm{E}+02$ \\
\hline Tc 99 & $1.92 \mathrm{E}+01$ & $4.84 \mathrm{E}-03$ & $1.00 \mathrm{E}+02$ \\
\hline Eu152 & $4.09 \mathrm{E}+00$ & $1.03 \mathrm{E}-03$ & $1.00 \mathrm{E}+02$ \\
\hline Sn121m & $2.94 \mathrm{E}+00$ & $7.41 \mathrm{E}-04$ & $1.00 \mathrm{E}+02$ \\
\hline Ce144 & $2.31 \mathrm{E}+00$ & $5.82 \mathrm{E}-04$ & $1.00 \mathrm{E}+02$ \\
\hline Pr144 & $2.31 \mathrm{E}+00$ & $5.82 \mathrm{E}-04$ & $1.00 \mathrm{E}+02$ \\
\hline Sn121 & $2.28 \mathrm{E}+00$ & $5.74 \mathrm{E}-04$ & $1.00 \mathrm{E}+02$ \\
\hline Zr 93 & $1.74 \mathrm{E}+00$ & $4.38 \mathrm{E}-04$ & $1.00 \mathrm{E}+02$ \\
\hline Pm146 & $1.32 \mathrm{E}+00$ & $3.32 \mathrm{E}-04$ & $1.00 \mathrm{E}+02$ \\
\hline Se 79 & $1.00 \mathrm{E}+00$ & $2.52 \mathrm{E}-04$ & $1.00 \mathrm{E}+02$ \\
\hline Nb 93m & $8.96 \mathrm{E}-01$ & $2.26 \mathrm{E}-04$ & $1.00 \mathrm{E}+02$ \\
\hline Sn126 & $8.40 \mathrm{E}-01$ & $2.12 \mathrm{E}-04$ & $1.00 \mathrm{E}+02$ \\
\hline Sb126m & $8.40 \mathrm{E}-01$ & $2.12 \mathrm{E}-04$ & $1.00 \mathrm{E}+02$ \\
\hline Cs135 & $7.10 \mathrm{E}-01$ & $1.79 \mathrm{E}-04$ & $1.00 \mathrm{E}+02$ \\
\hline Pd107 & $1.86 \mathrm{E}-01$ & $4.69 \mathrm{E}-05$ & $1.00 \mathrm{E}+02$ \\
\hline Pm145 & $1.31 \mathrm{E}-01$ & $3.30 \mathrm{E}-05$ & $1.00 \mathrm{E}+02$ \\
\hline Sb126 & $1.18 \mathrm{E}-01$ & $2.97 \mathrm{E}-05$ & $1.00 \mathrm{E}+02$ \\
\hline Rh102 & $8.58 \mathrm{E}-02$ & $2.16 \mathrm{E}-05$ & $1.00 \mathrm{E}+02$ \\
\hline Totals & $3.97 \mathrm{E}+05$ & & \\
\hline
\end{tabular}





\section{APPENDIX C}

Graphical Representations of the DORT Calculational Models

Used in the Shielding Analysis of the SNF Shipping Casks 


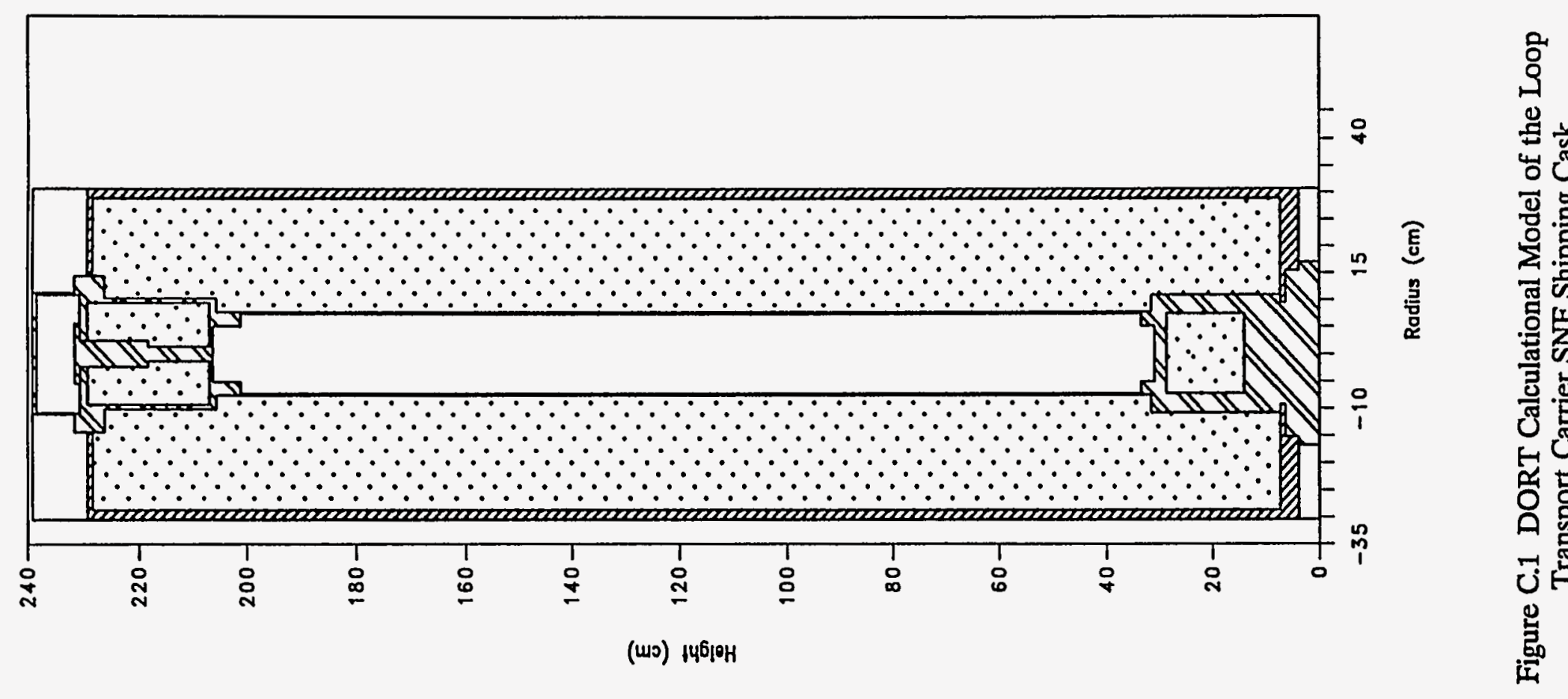




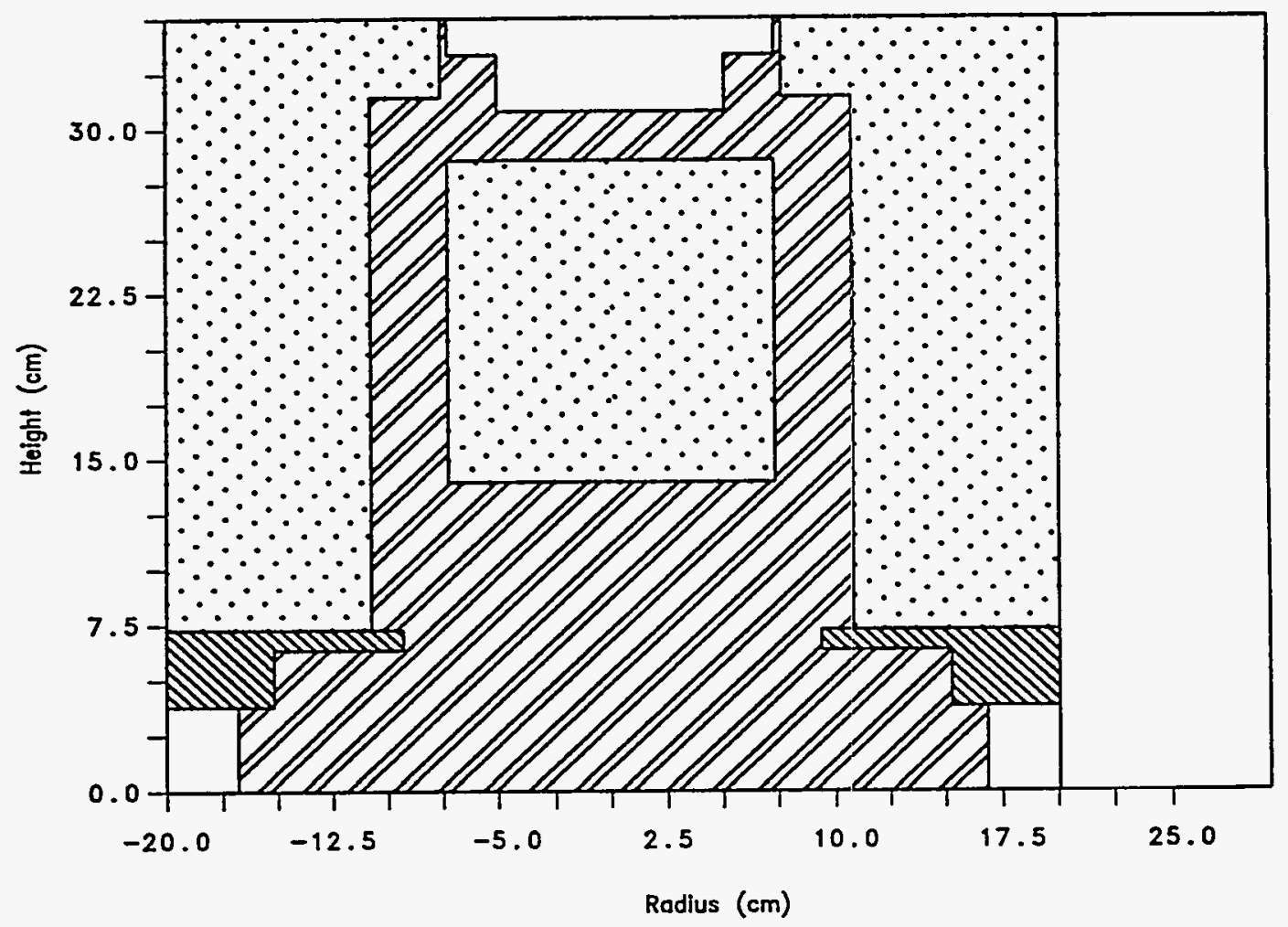

Figure C.2 Expanded View of the DORT Calculational Model of the Loop Transport Carrier Door Plug.

C-4 


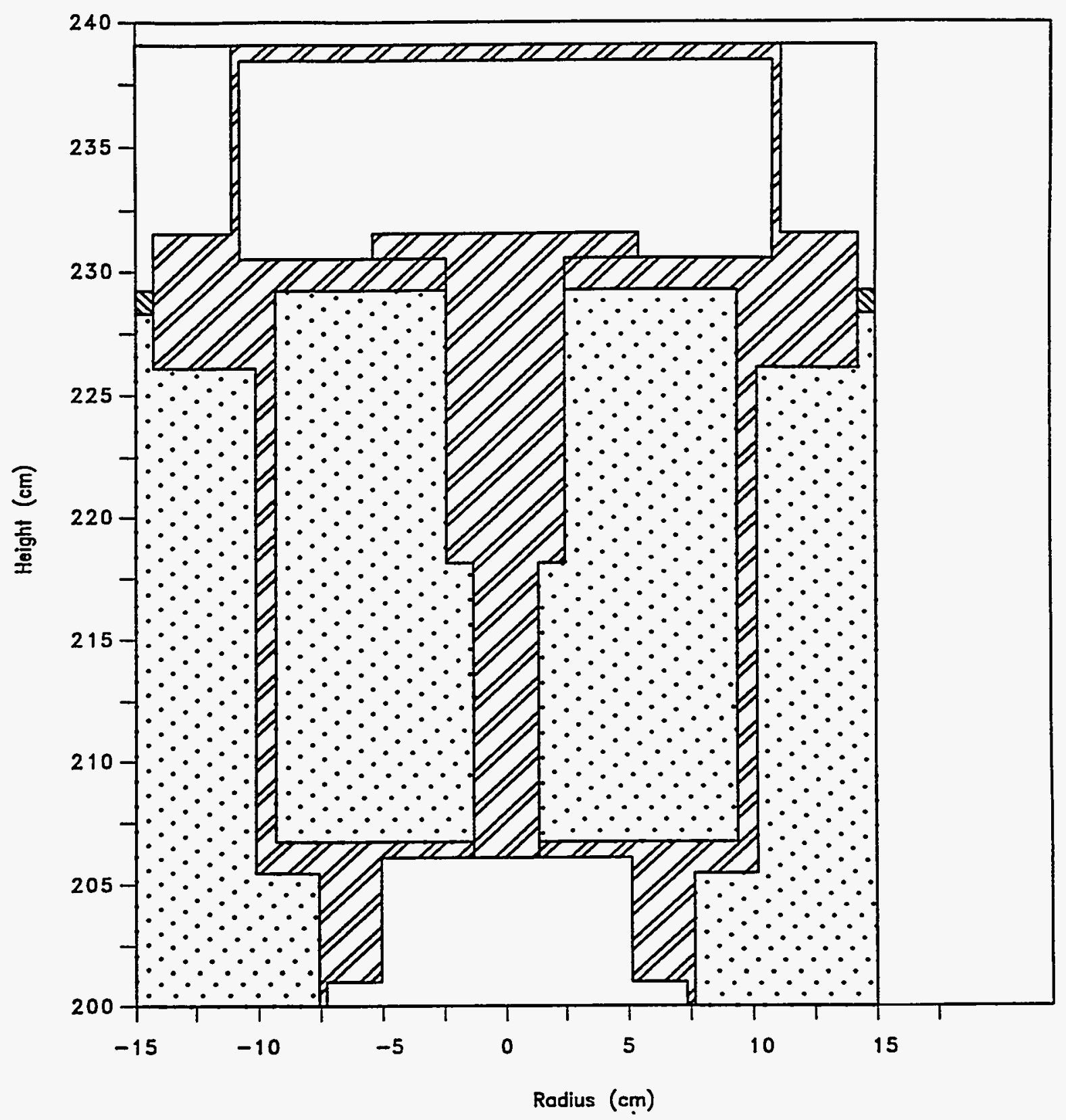

Figure C.3 Expanded View of the DORT Calculational Model of the Loop Transport Carrier End Plug. 


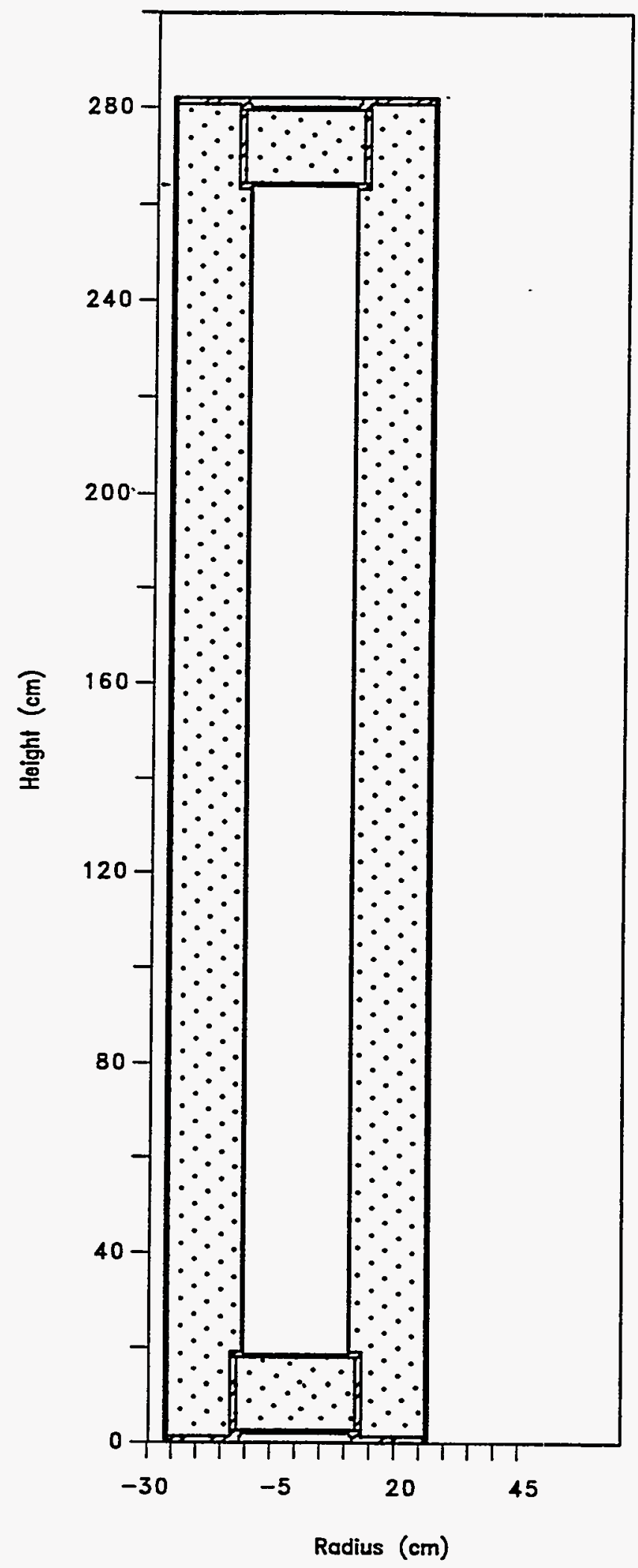

Figure C.4 DORT Calculational Model of the In-Pile Loop LITR HB-2 Carrier SNF Shipping Cask. 


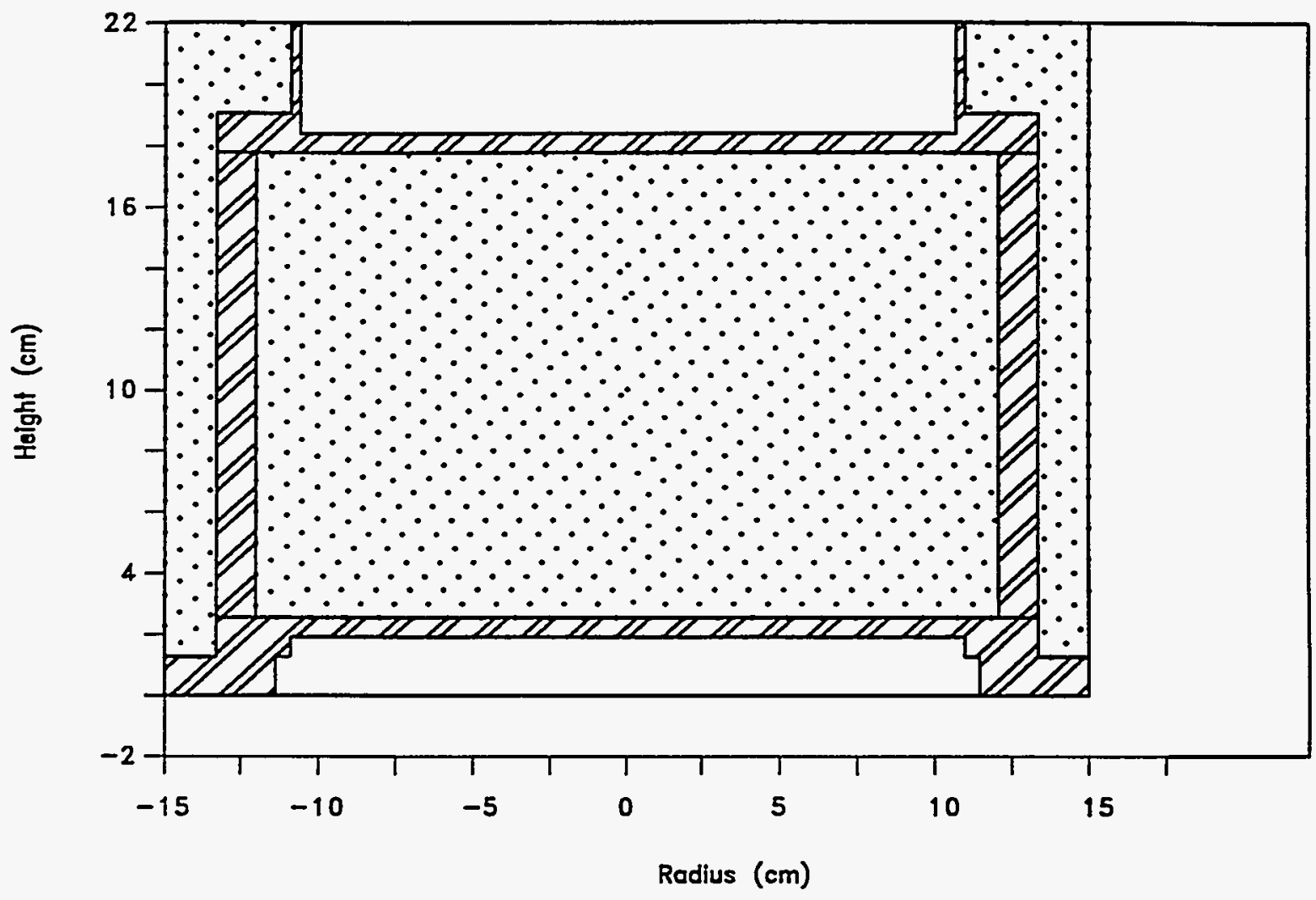

Figure C.5 Expanded View of the DORT Calculational Model of the In-Pile Loop LITR HB-2 Carrier Double Doors. 


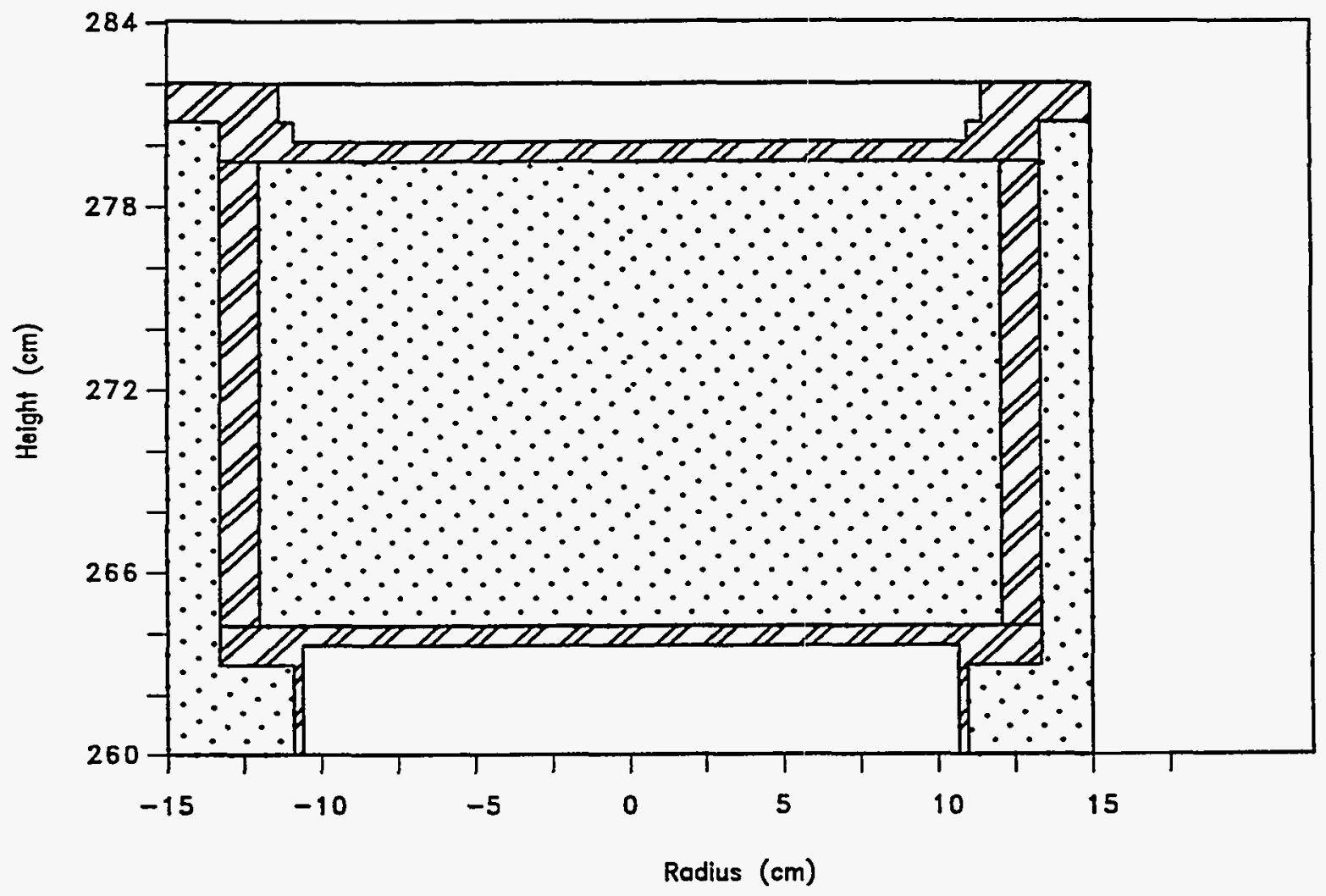

Figure C.6 Expanded View of the DORT Calculational Model of the In-Pile Loop LITR HB-2 Carrier Large Door. 


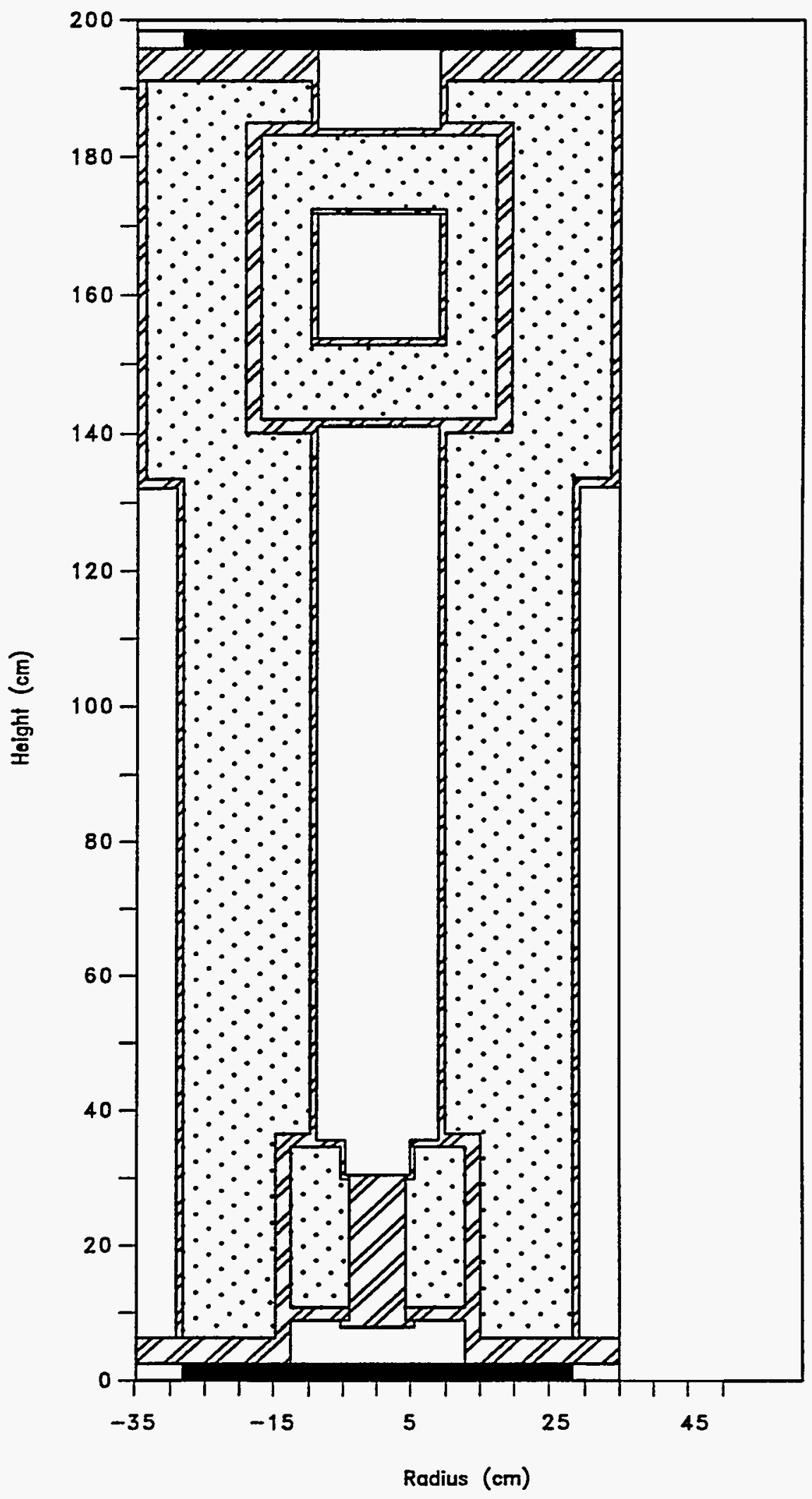

Figure C.7 DORT Calculational Model of the 6.5 Inch HRLEL Carrier SNF Shipping Cask. 


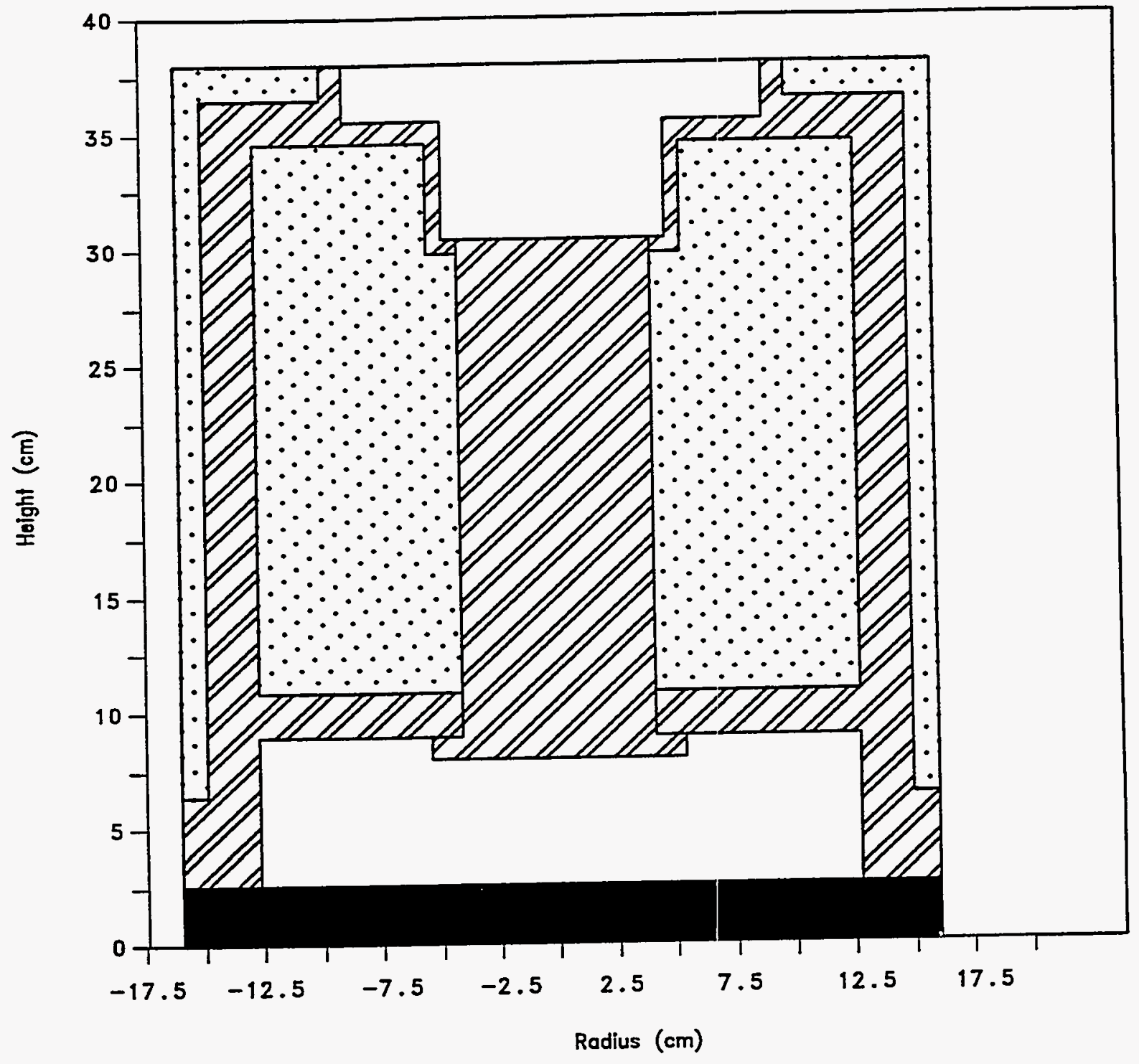

Figure C.8 Expanded View of the DORT Calculational Model of the 6.5 Inch HRLEL Carrier End Plug. 


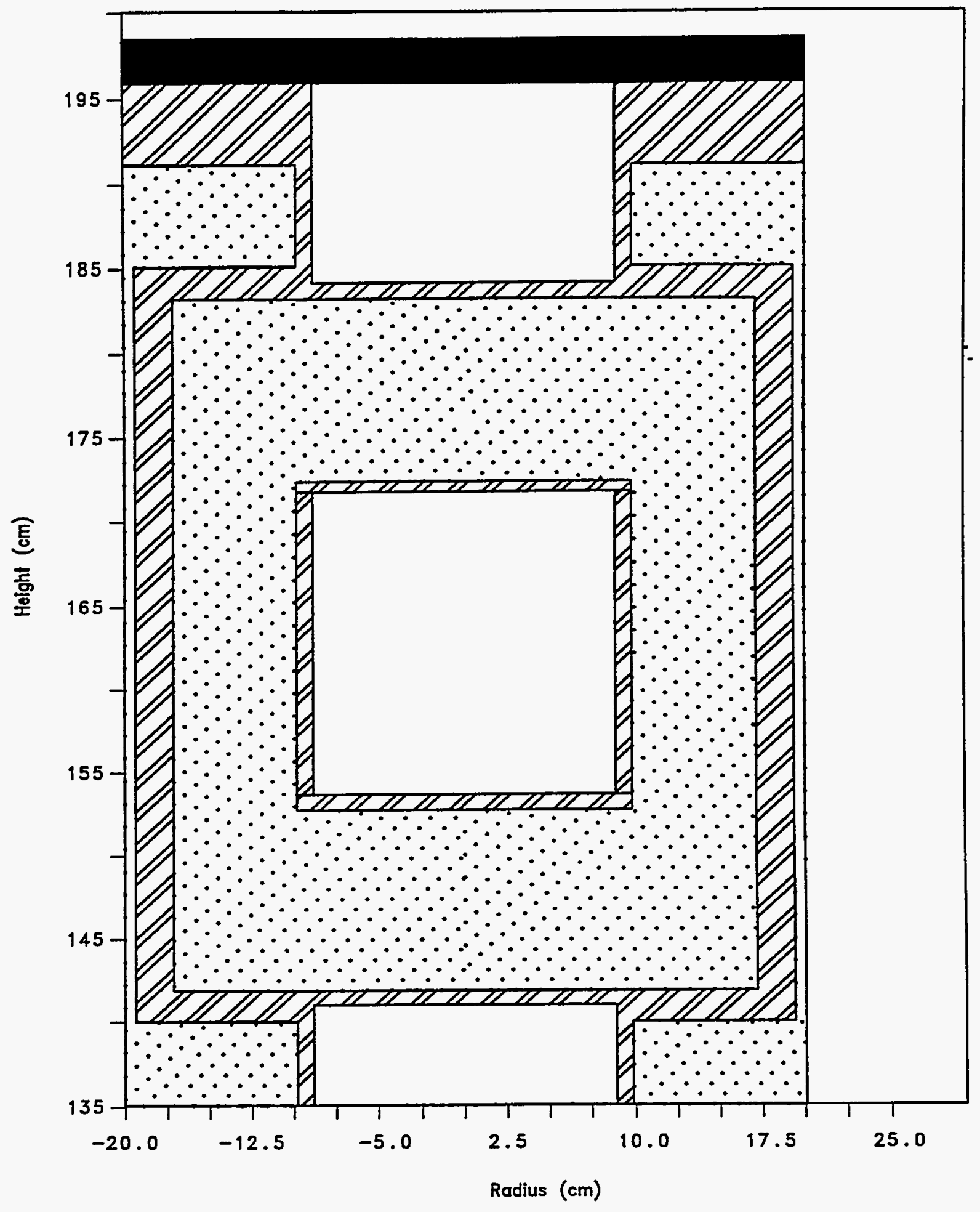

Figure C.9 Expanded View of the DORT Calculational Model of the 6.5 Inch HRLEL Carrier Door. 


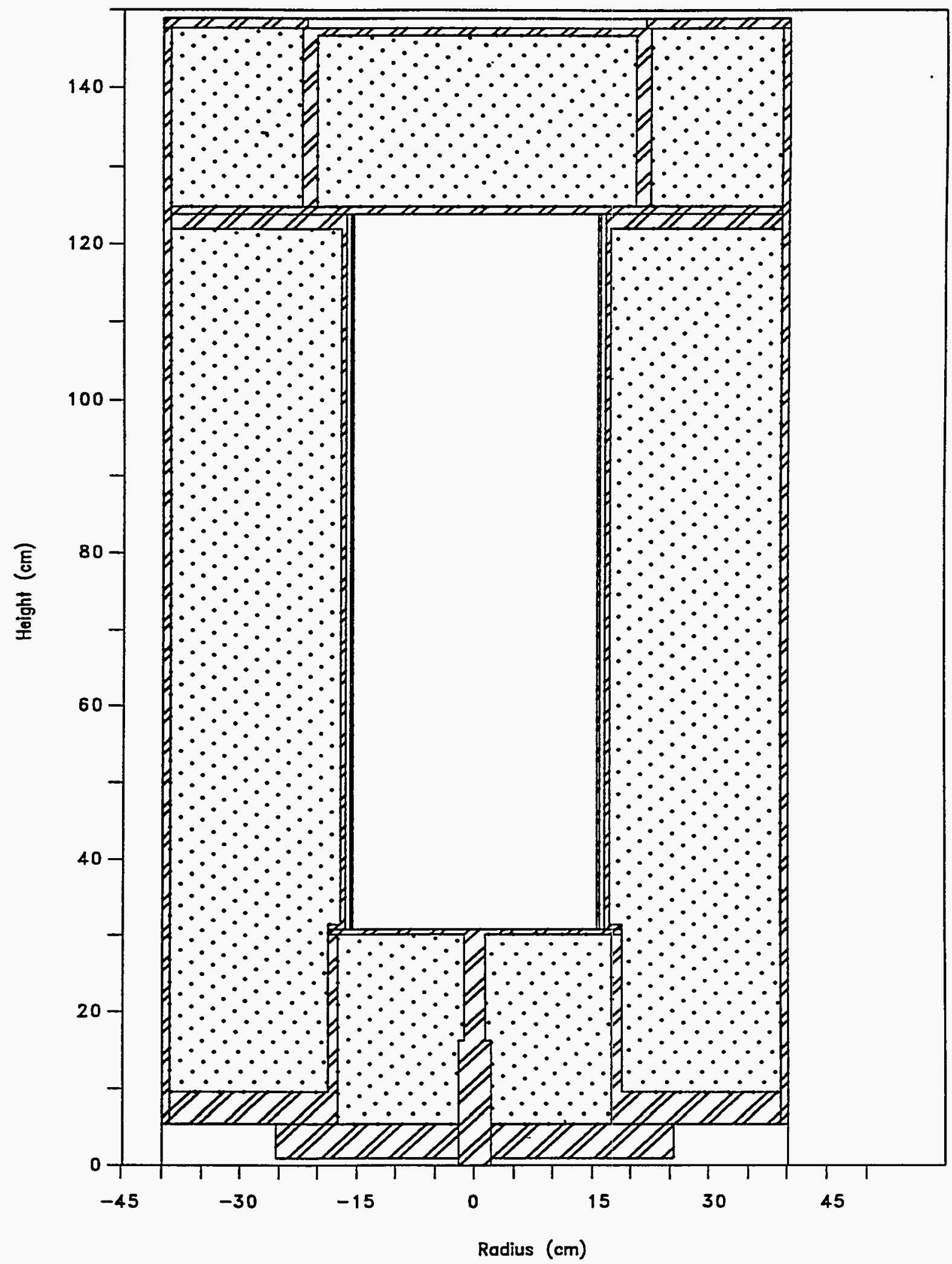

Figure C.10 DORT Calculational Model of the HFIR Hot Scrap Carrier SNF Shipping Cask. 


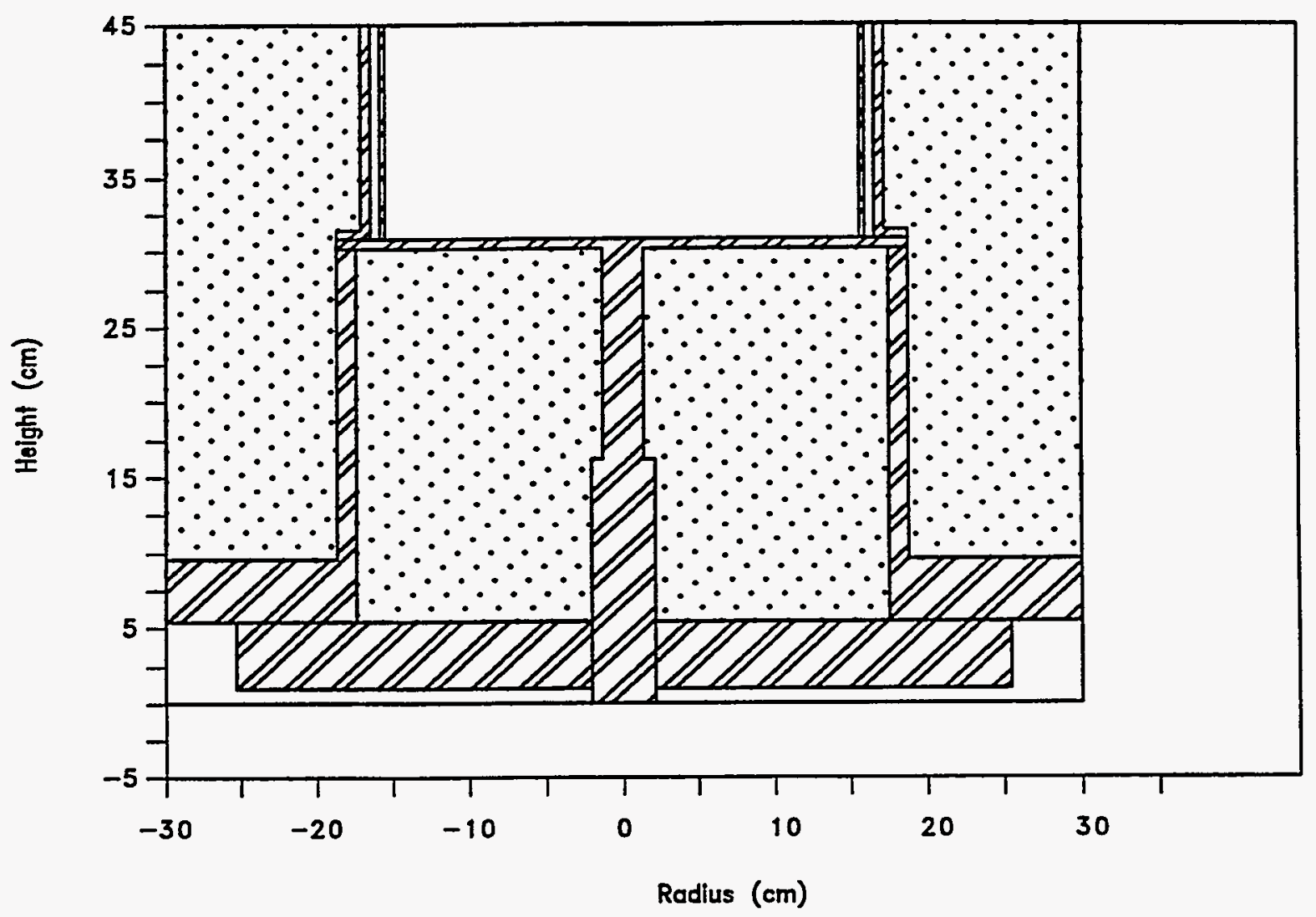

Figure C.11 Expanded View of the DORT Calculational Model of the HFIR Hot Scrap Carrier Top Plug.

\section{C-13}




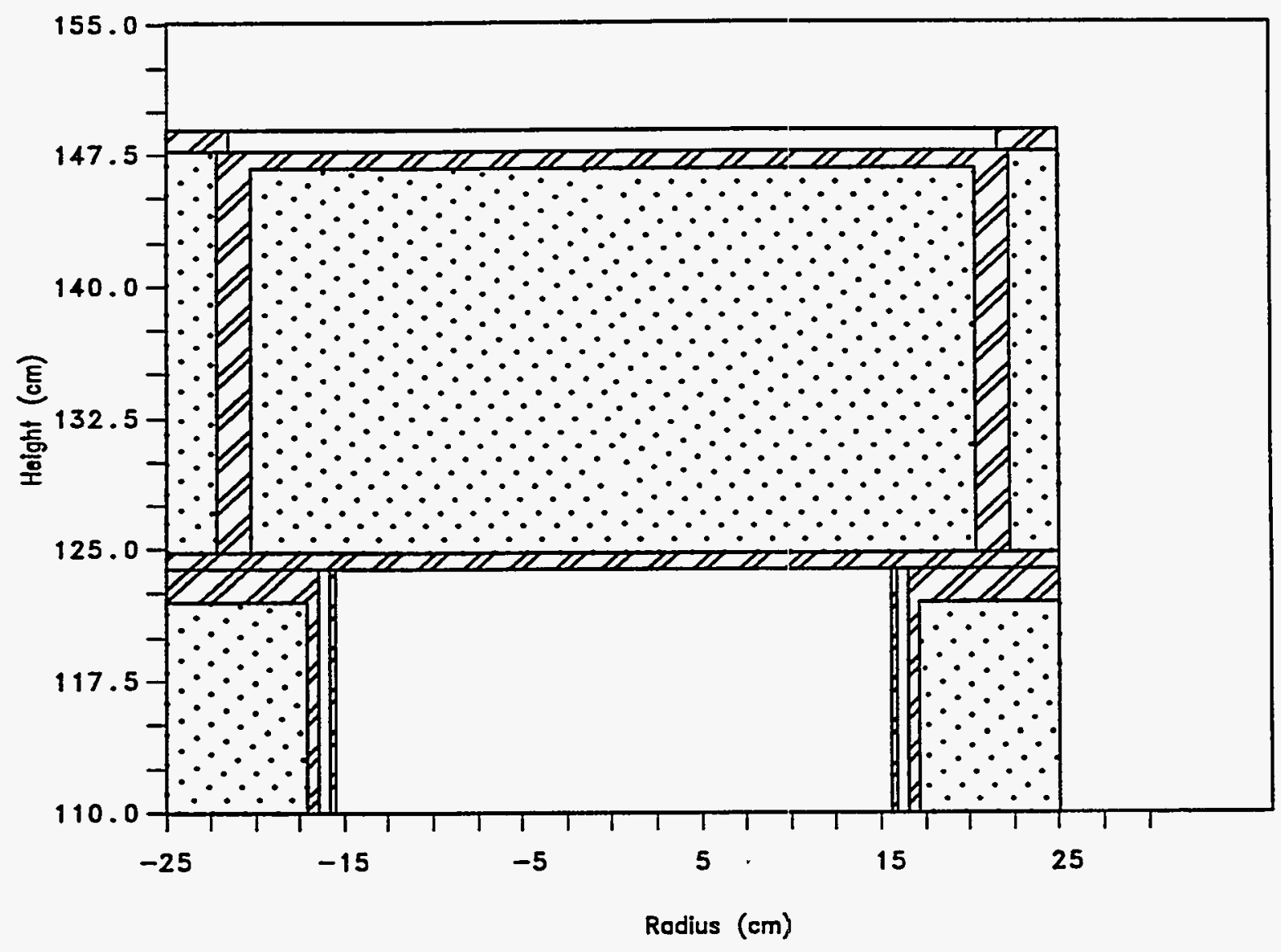

Figure C.12 Expanded View of the DORT Calculational Model of the HFIR Hot Scrap Carrier Bottom Door. 


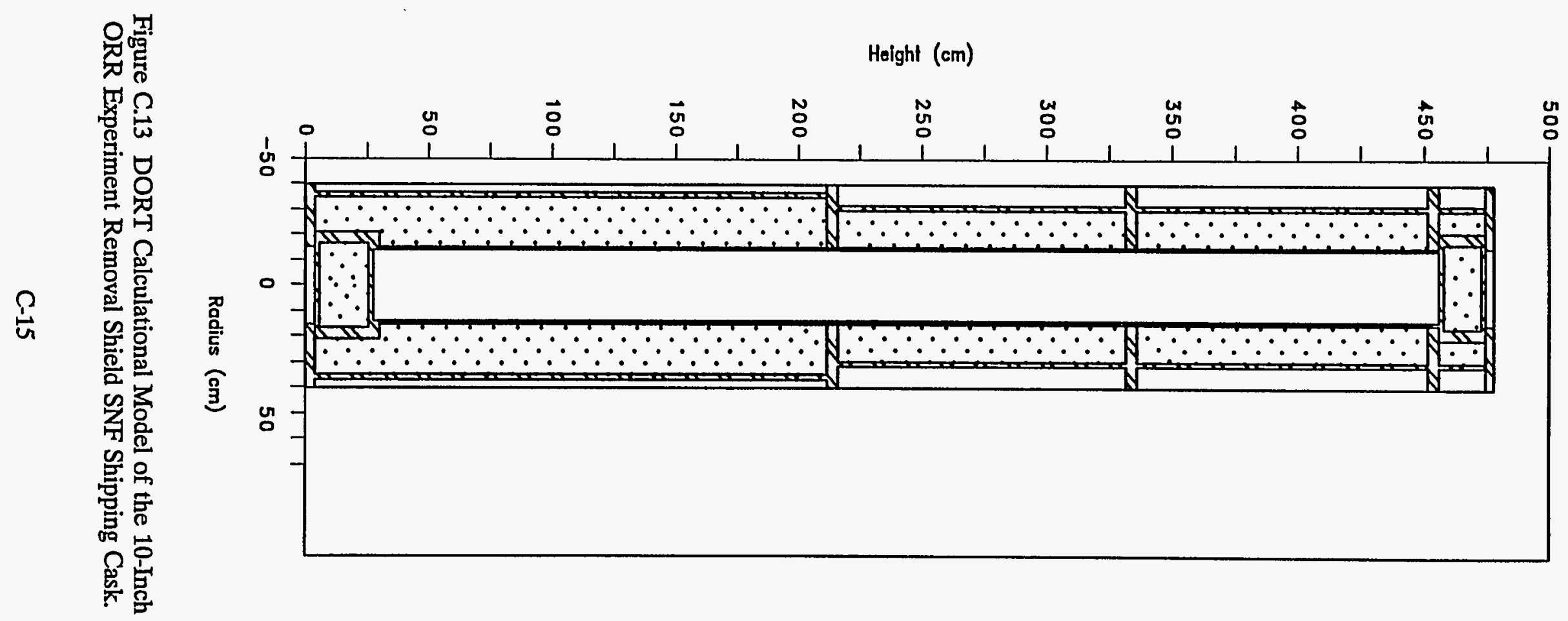




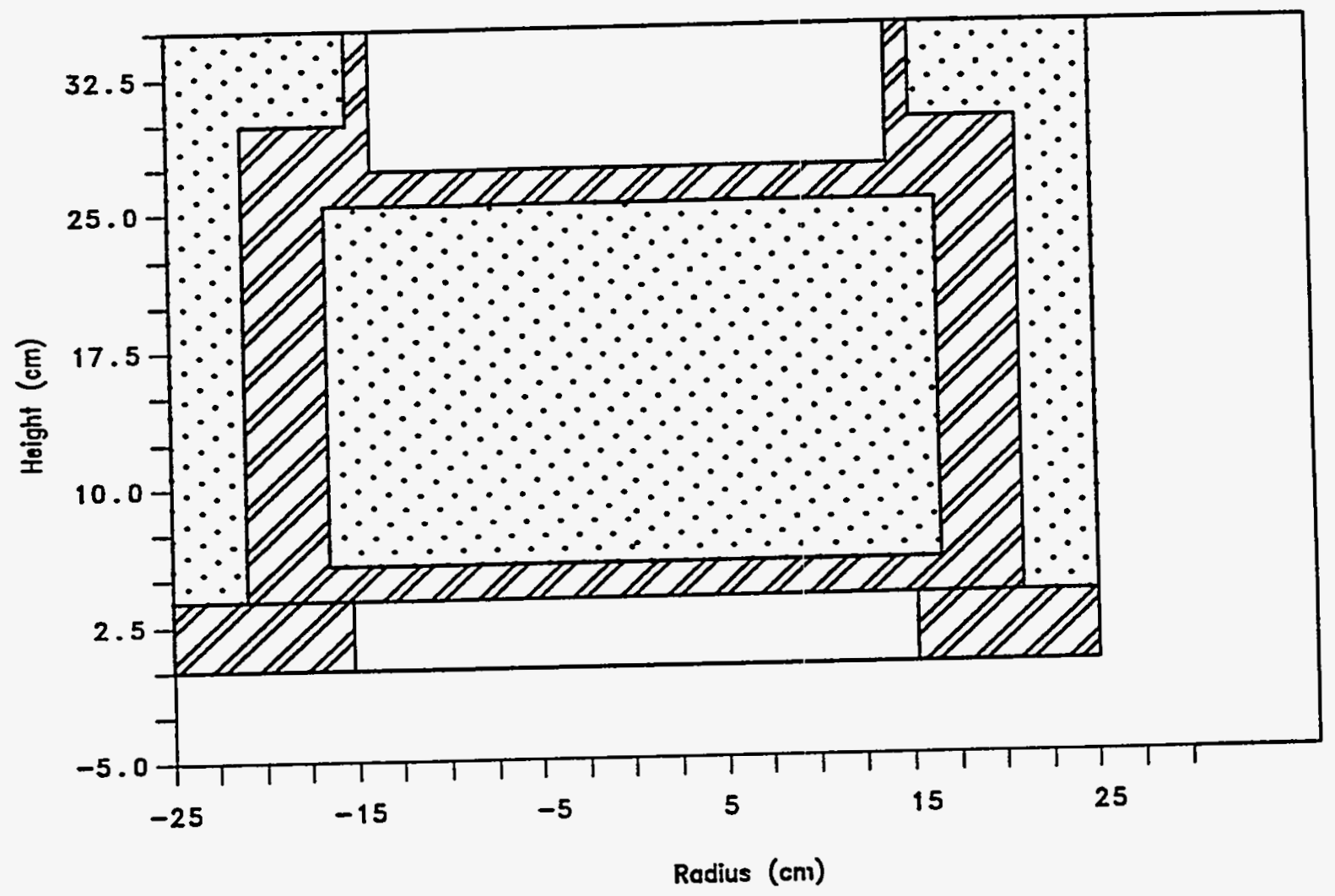

Figure C.14 Expanded View of the DORT Calculational Model of the 10-Inch ORR Experiment Removal Shield Heavy Door. 


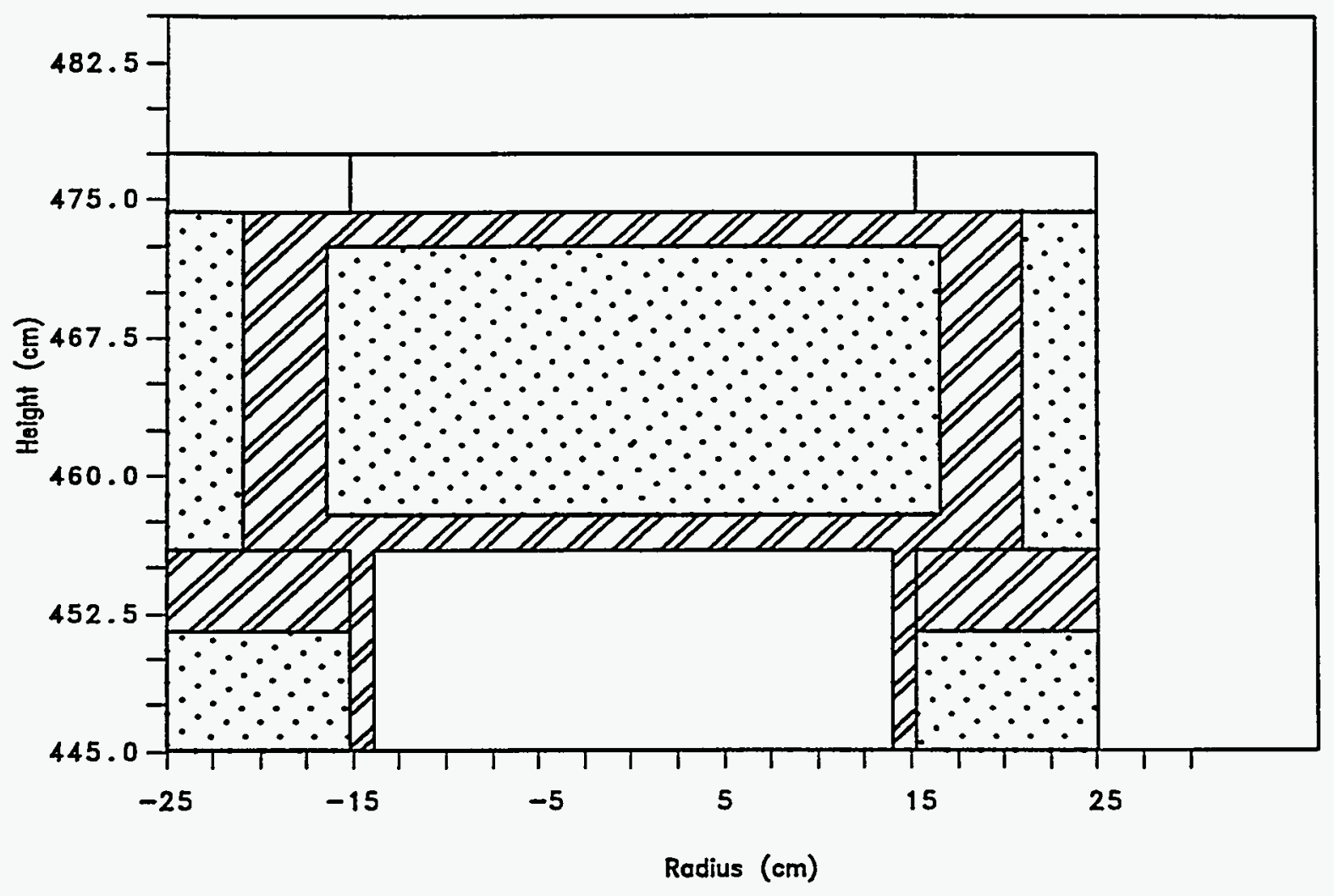

Figure C.15 Expanded View of the DORT Calculational Model of the 10-Inch ORR Experiment Removal Shield Light Door. 



\section{APPENDIX D}

Isodose Contours for the Representative Sources Used in the Shielding Analysis of the SNF Shipping Casks 



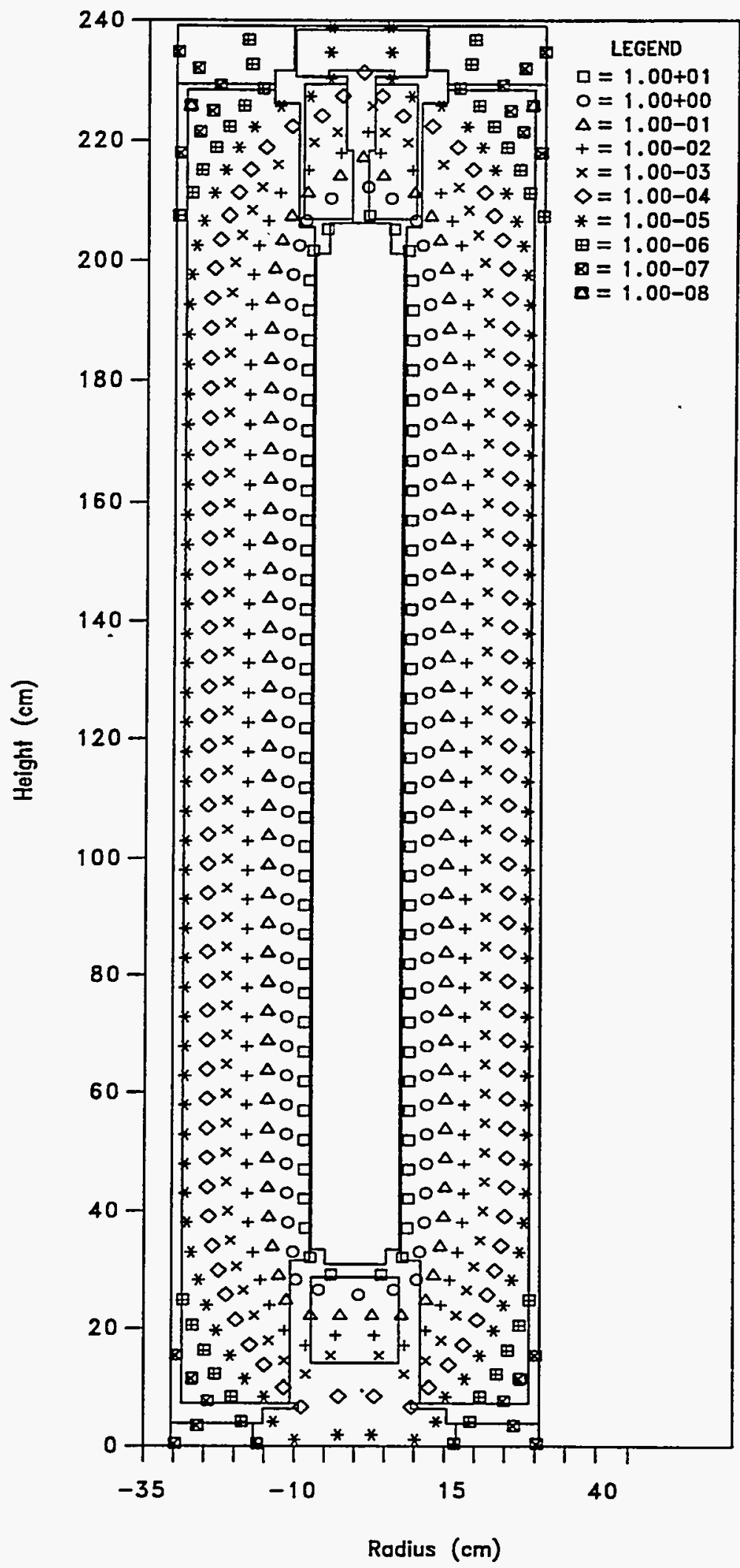

Figure D.1 Isodose Contours for the ${ }^{60} \mathrm{Co}$ Source Packaged in the Loop Transport Carrier SNF Shipping Cask. [Dose units are rem/(h-Ci).] 


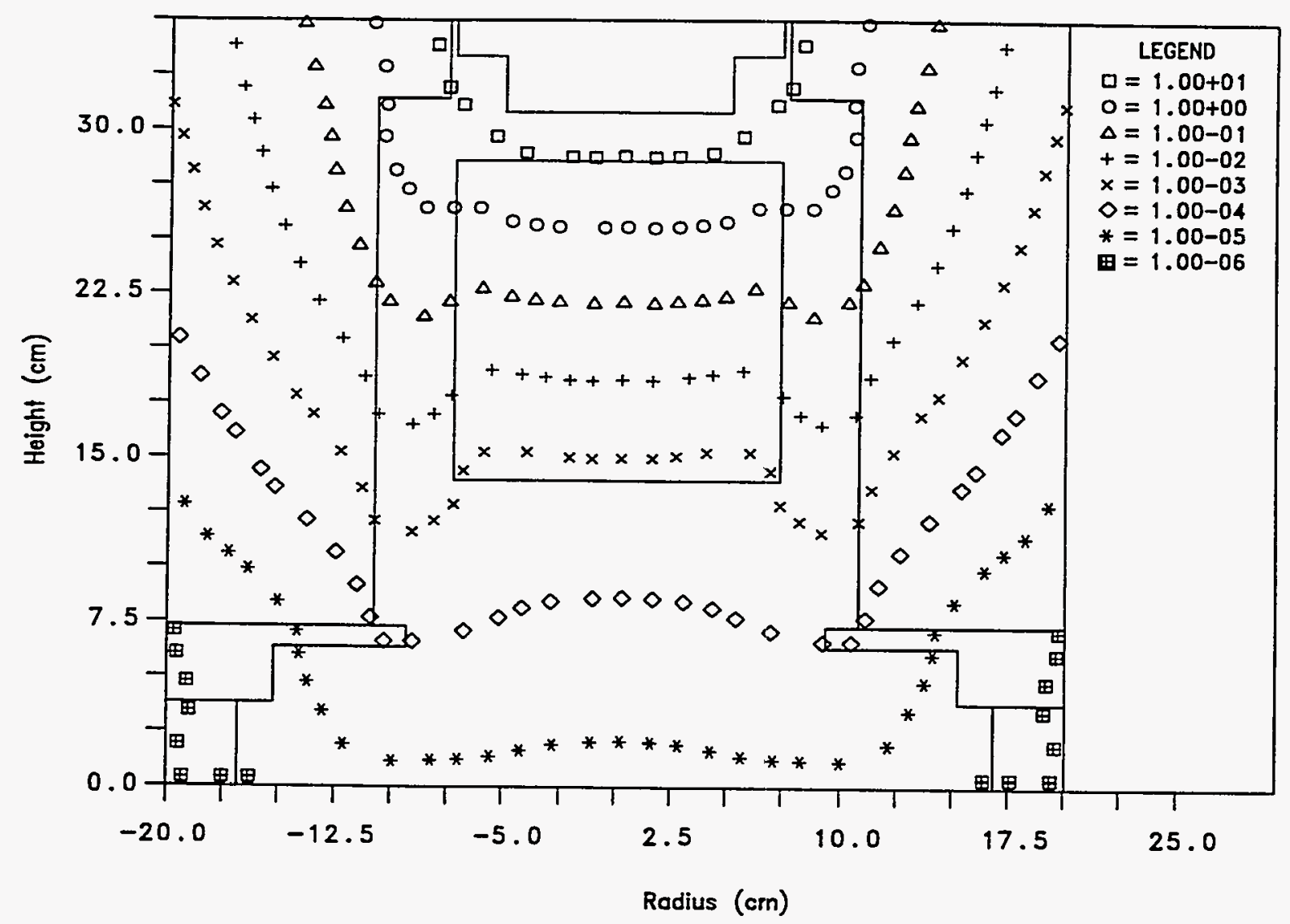

Figure D.2 Expanded View of the Door Plug Isodose Contours for the ${ }^{60} \mathrm{Co}$ Source Packaged in the Loop Transport Carrier SNF Shipping Cask.

[Dose units are $\mathrm{rem} /(\mathrm{h} \cdot \mathrm{Ci})$.]

D-4 


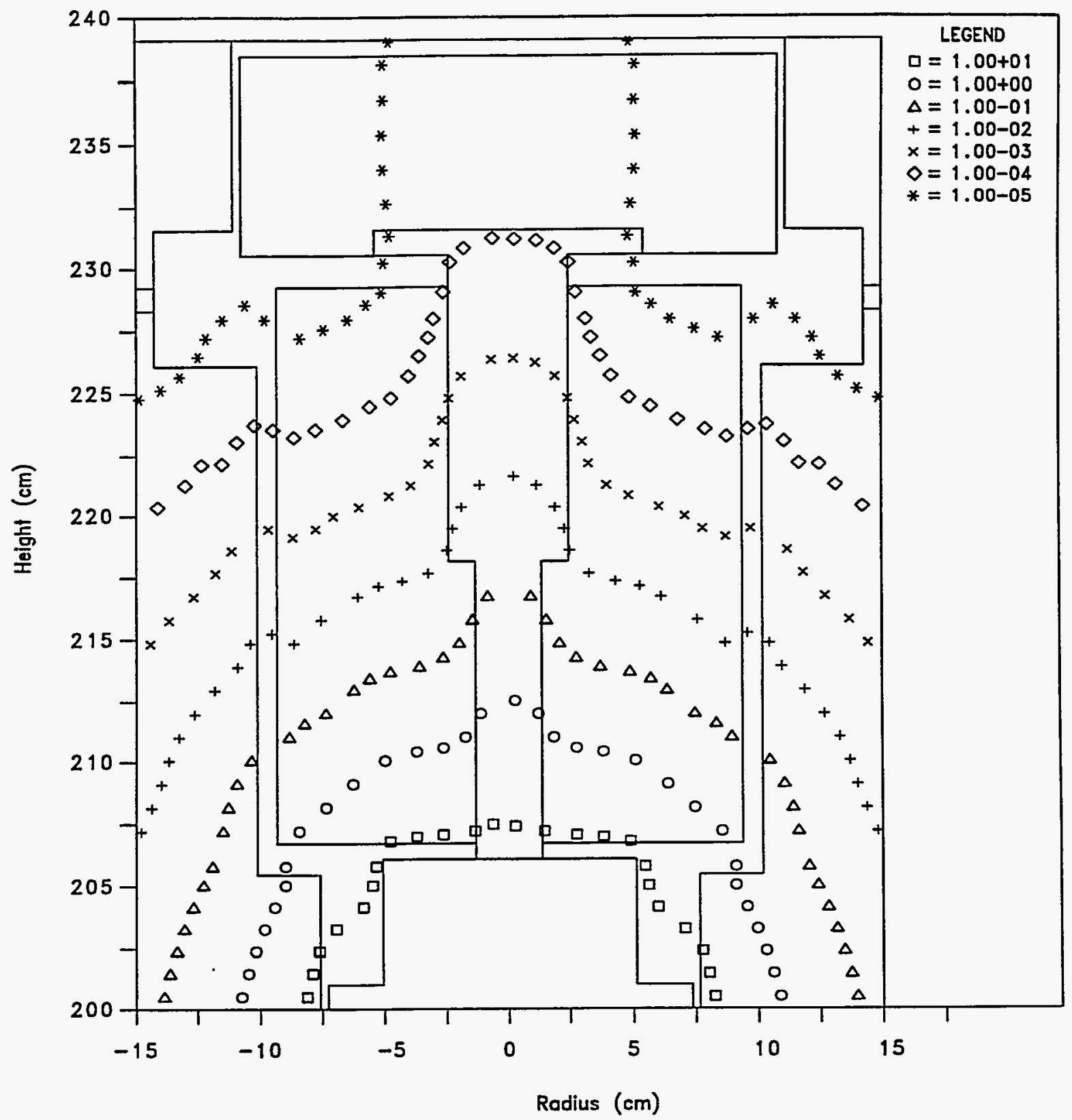

Figure D.3 Expanded View of the End Plug Isodose Contours for the ${ }^{60} \mathrm{Co}$ Source Packaged in the Loop Transport Carrier SNF Shipping Cask. [Dose units are rem $/ \mathrm{h} \cdot \mathrm{Ci})$.]

D-5 
Height (cm)

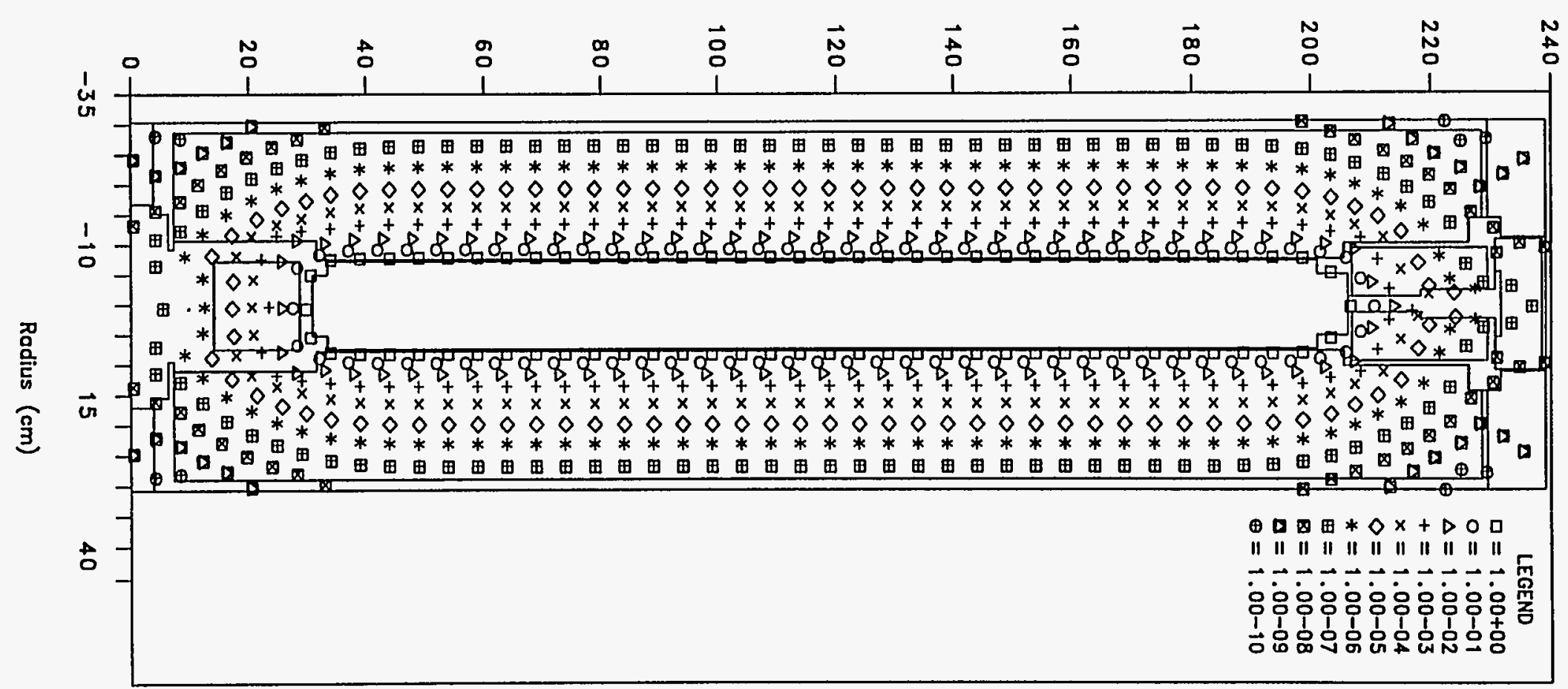




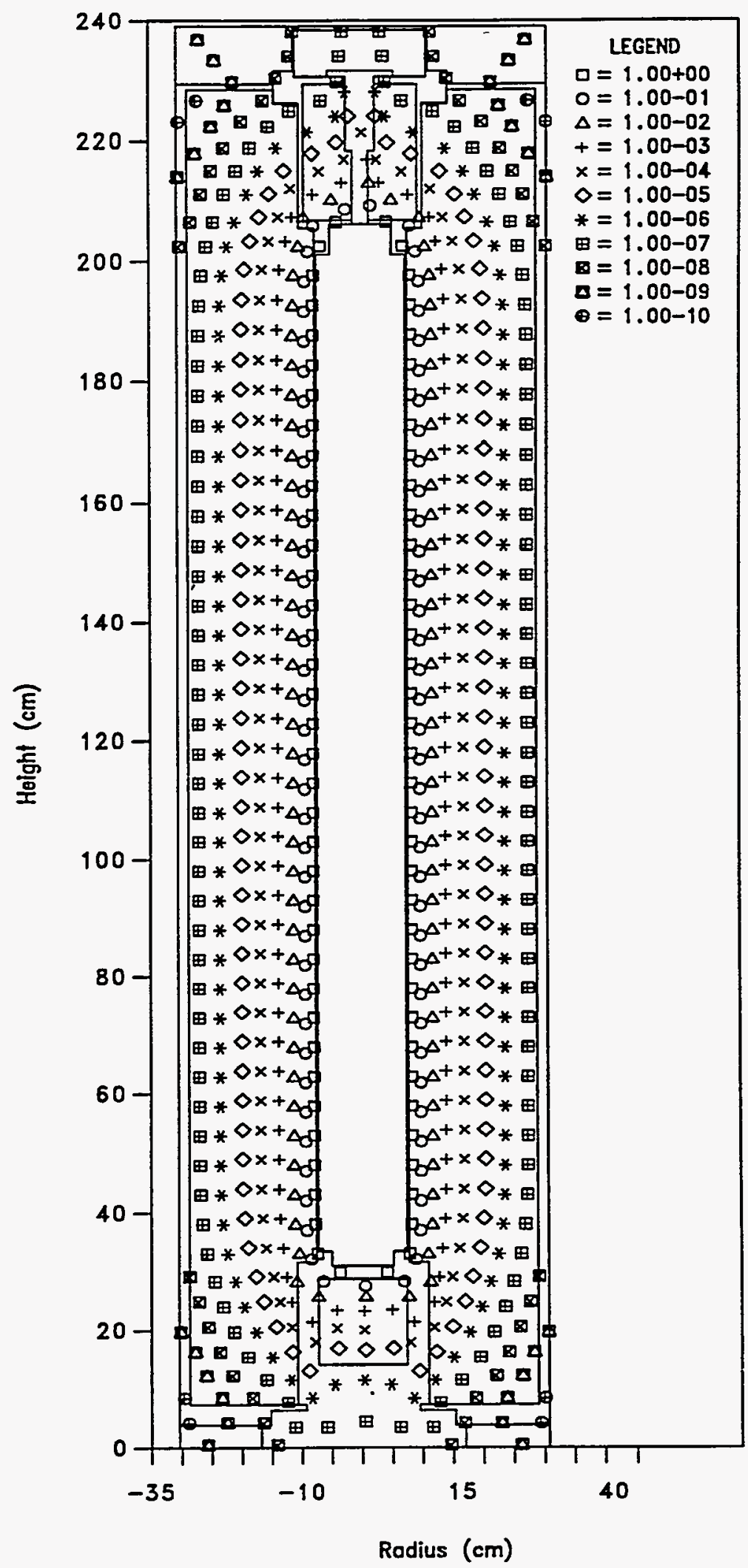

Figure D.5 Isodose Contours for the LWR MFP (50 MWd/kgU) Source Packaged in the Loop Transport Carrier SNF Shipping Cask.

[Dose units are rem/(h.Ci).]

D-7 


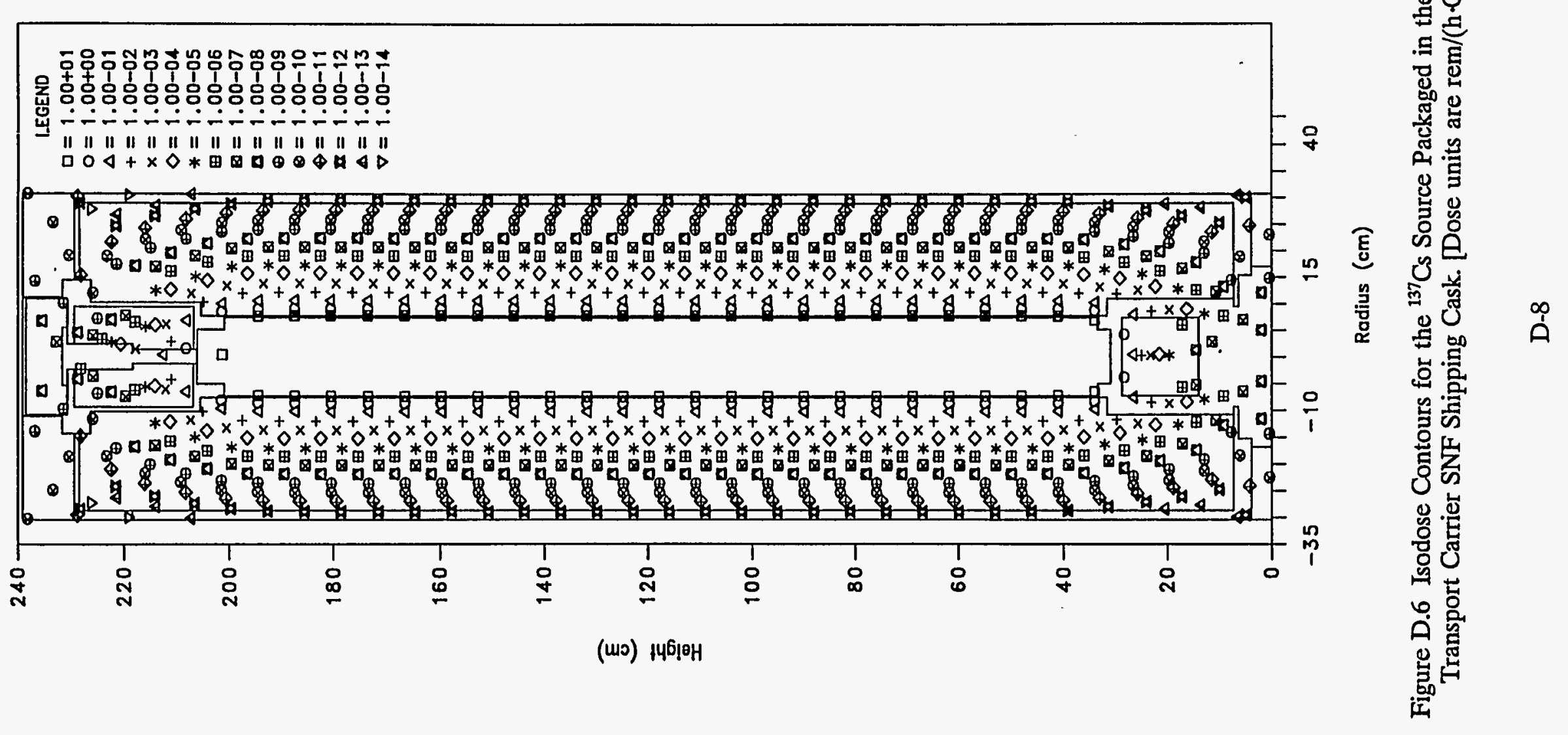




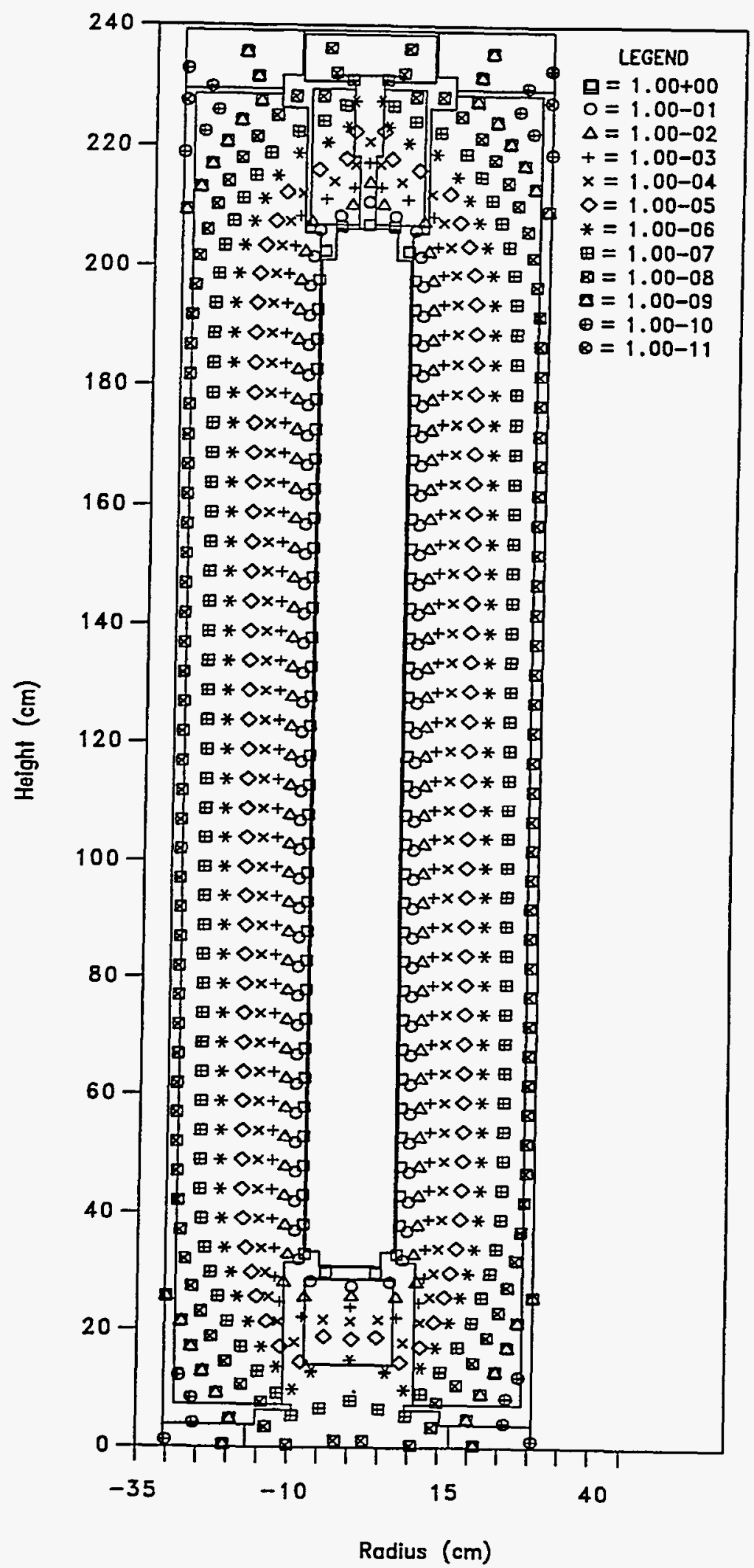

Figure D.7 Isodose Contours for the ORR MFP (20\% Enriched ${ }^{235} \mathrm{U}$ ) Source Packaged in the Loop Transport Carrier SNF Shipping Cask.

[Dose units are rem/(h-Ci).] 


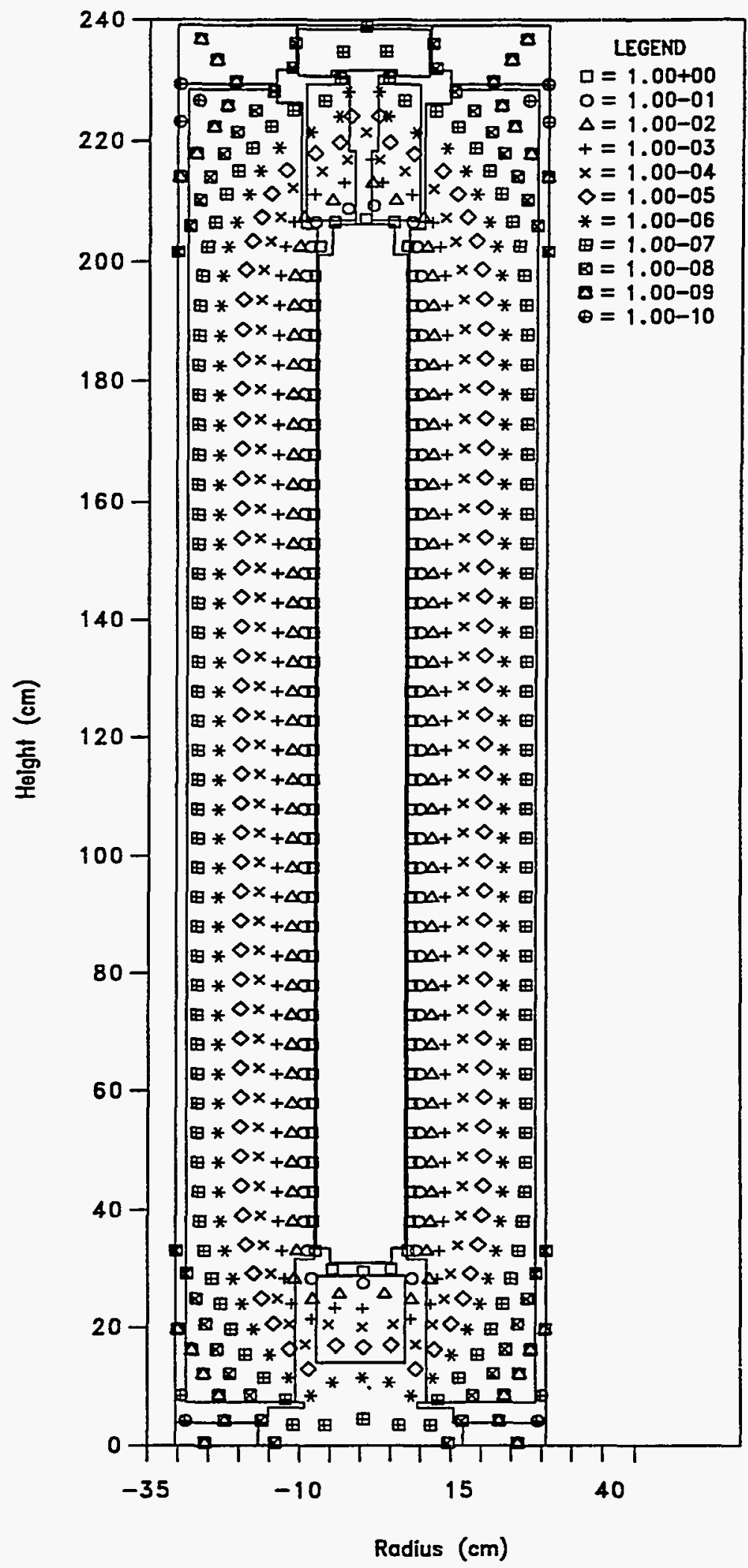

Figure D.8 Isodose Contours for the ORR MFP (40\% Enriched ${ }^{235} \mathrm{U}$ ) Source Packaged in the Loop Transport Carrier SNF Shipping Cask.

[Dose units are rem/(h-Ci).] 


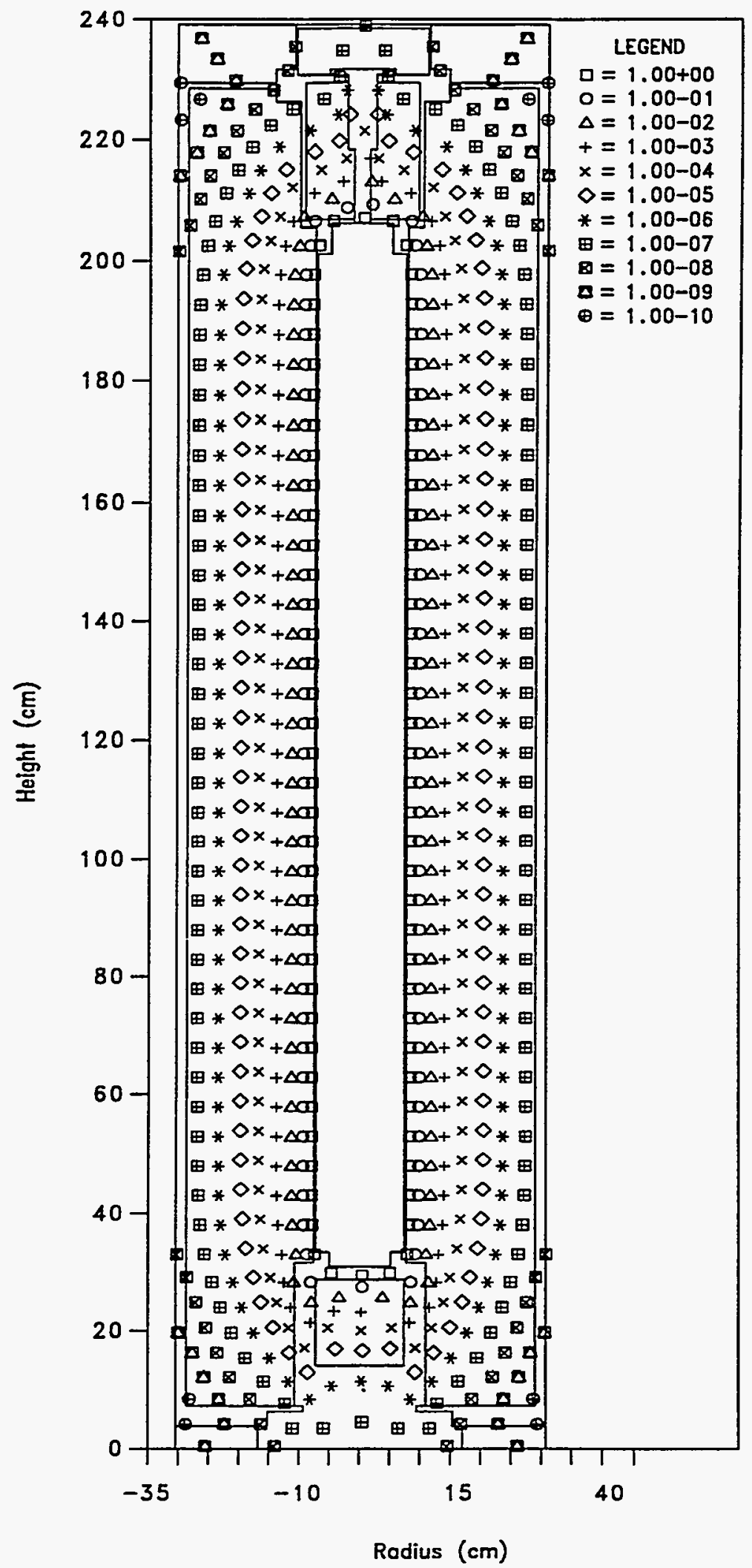

Figure D.9 Isodose Contours for the ORR MFP (93\% Enriched ${ }^{235} \mathrm{U}$ ) Source Packaged in the Loop Transport Carrier SNF Shipping Cask. [Dose units are rem/(h-Ci).] 


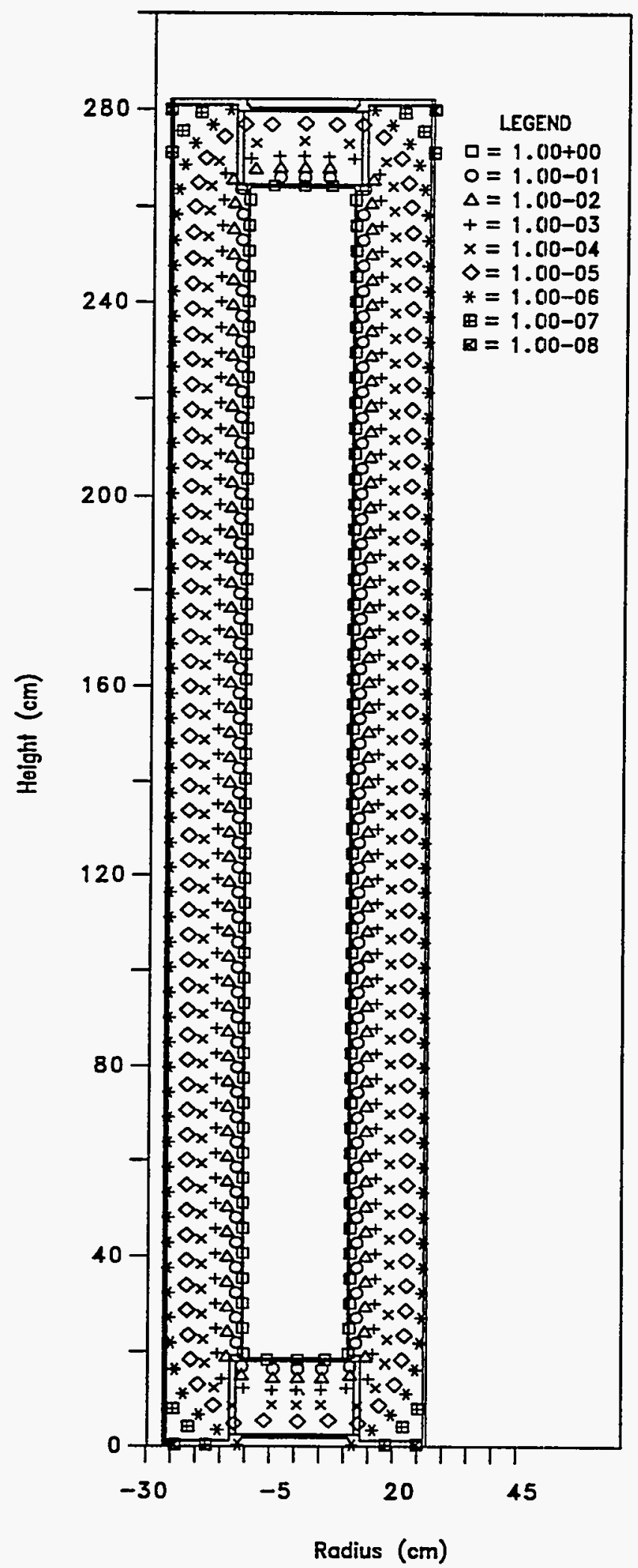

Figure D.10 Isodose Contours for the 60Co Source Packaged in the In-Pile Loop LITR HB-2 Carrier SNF Shipping Cask.

[Dose units are rem/(h-Ci).]

D-12 


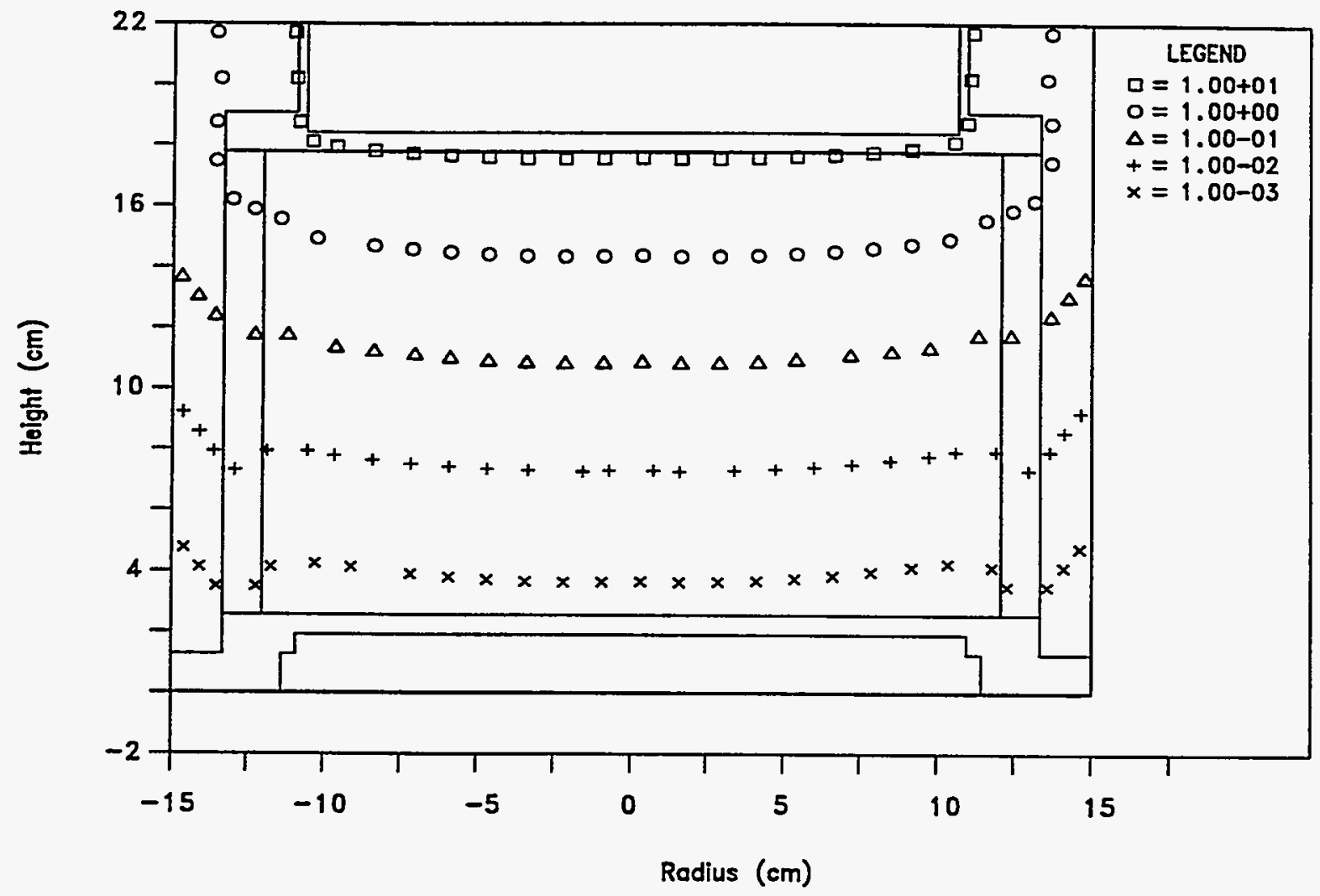

Figure D.11 Expanded View of the Double Doors Isodose Contours for the ${ }^{60} \mathrm{Co}$ Source Packaged in the In-Pile Loop LITR HB-2 Carrier SNF

Shipping Cask. [Dose units are rem/(h-Ci).]

\section{D-13}




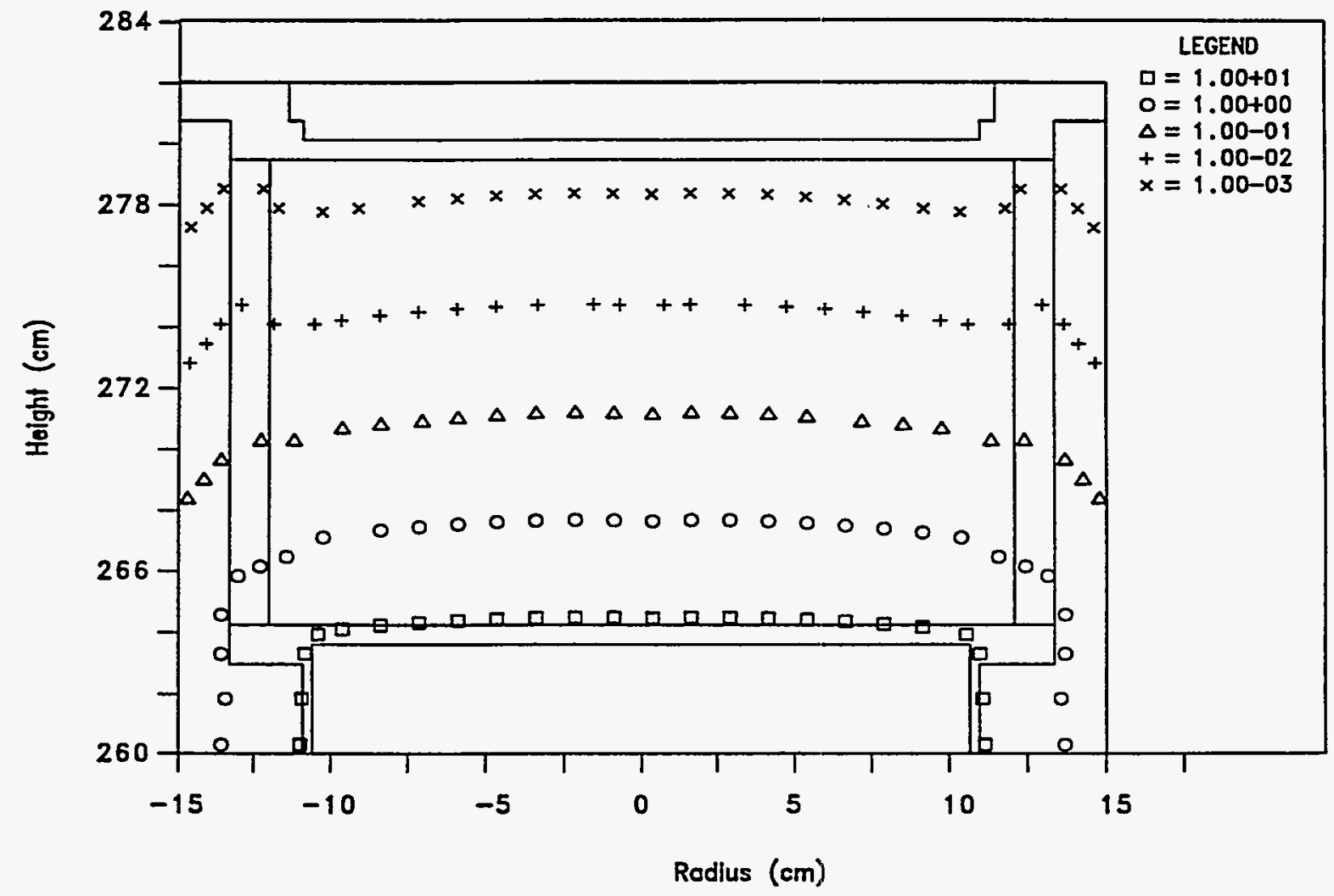

Figure D.12 Expanded View of the Large Door Isodose Contours for the ${ }^{60} \mathrm{Co}$ Source Packaged in the In-Pile Loop LITR HIB-2 Carrier SNF

Shipping Cask. [Dose units are rem/(h·Ci).]

D-14 


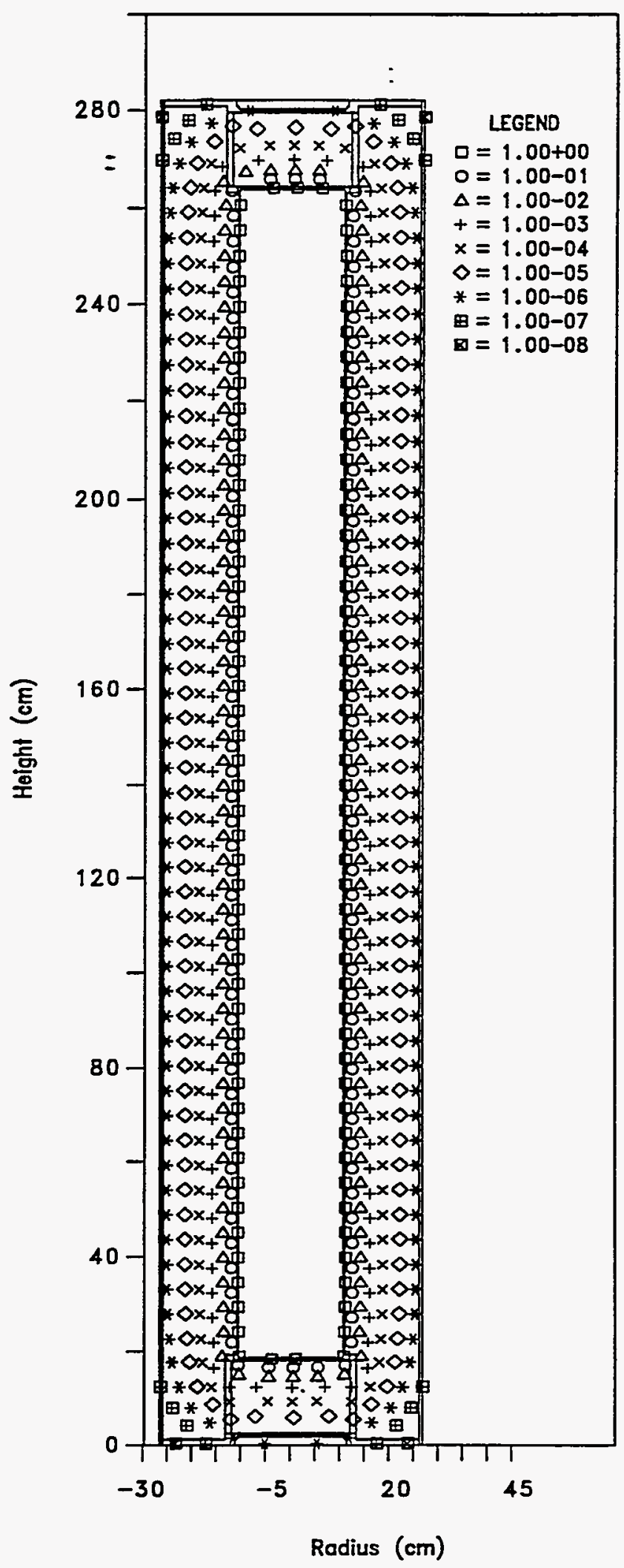

Figure D.13 Isodose Contours for the LWR MFP (25 MWd/kgU) Source Packaged in the In-Pile Loop LITR HB-2 Carrier SNF Shipping Cask. [Dose units are rem/h-Ci).]

\section{D-15}




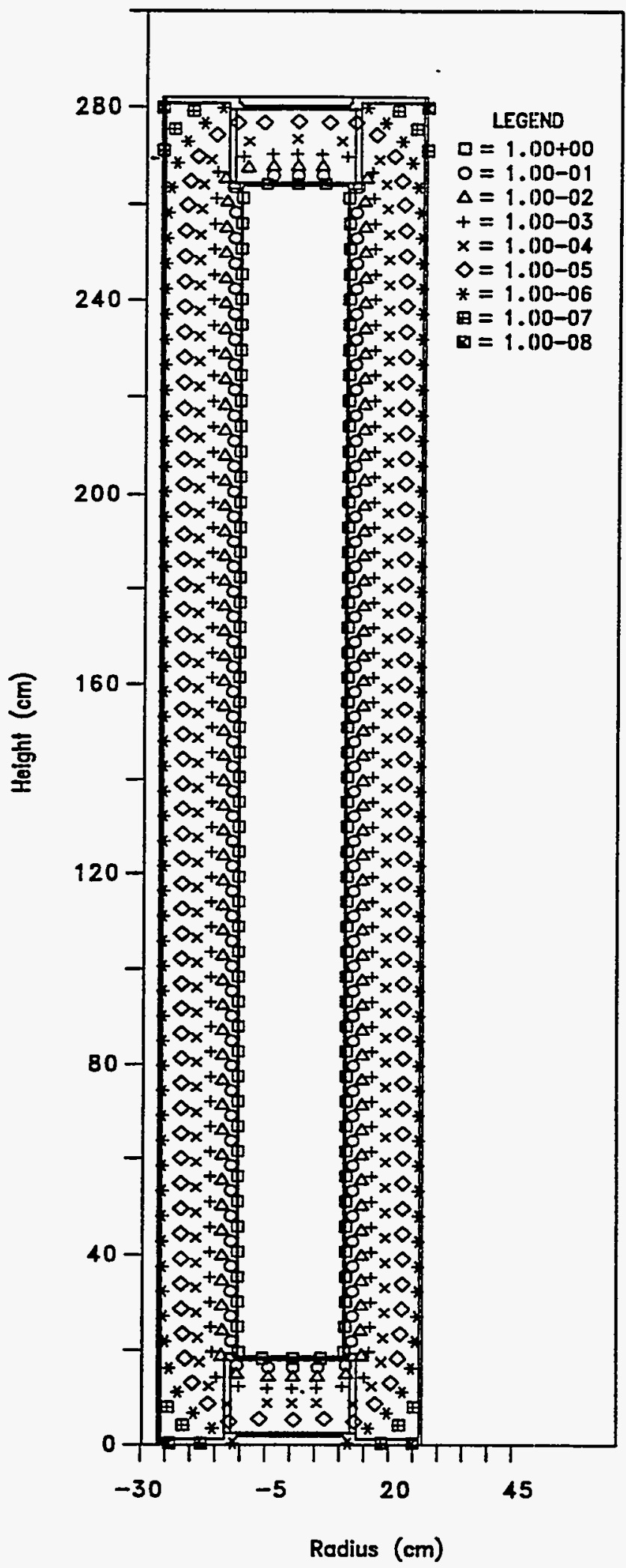

Figure D.14 Isodose Contours for the LWR MFP (50 MWd/kgU) Source Packaged in the In-Pile Loop LITR HB-2 Carrier SNF Shipping Cask. [Dose units are rem/(h.Ci).] 


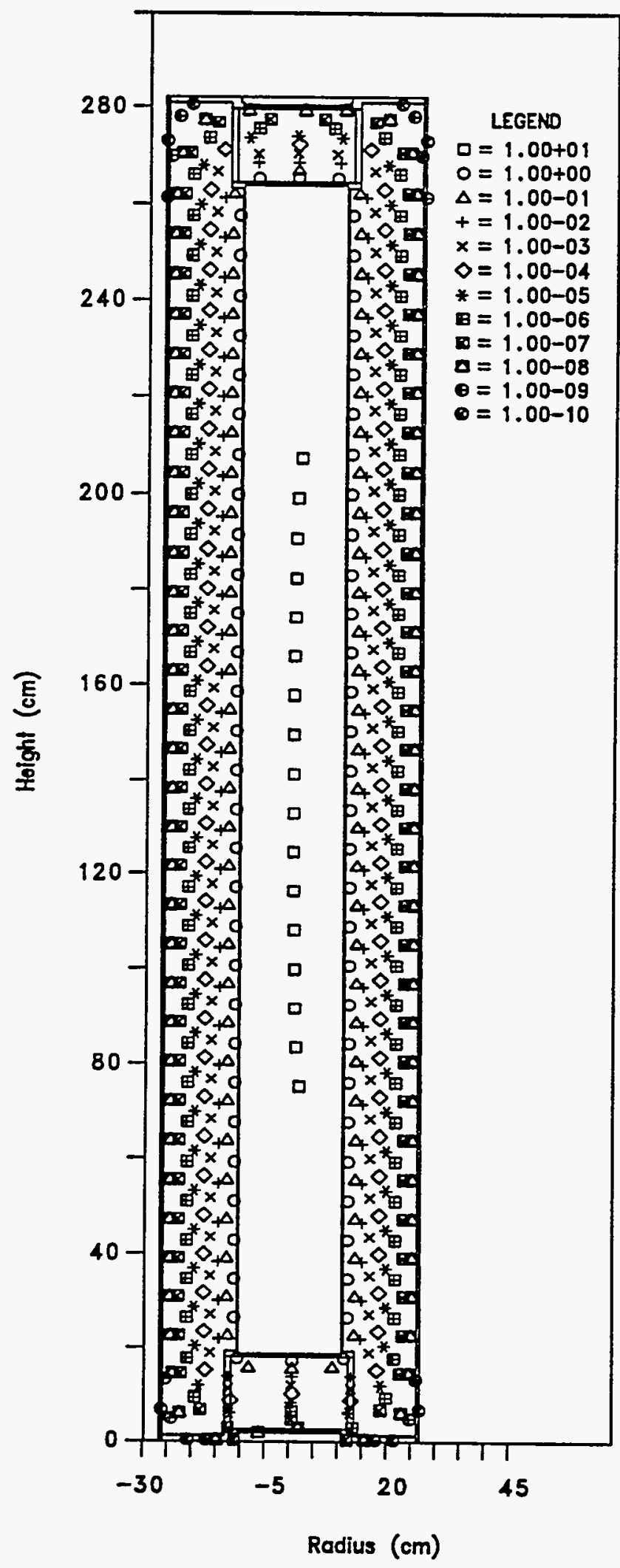

Figure D.15 Isodose Contours for the ${ }^{137} \mathrm{Cs}$ Source Packaged in the In-Pile Loop LITR HB-2 Carrier SNF Shipping Cask.

[Dose units are rem/(h.Ci).]

D-17 


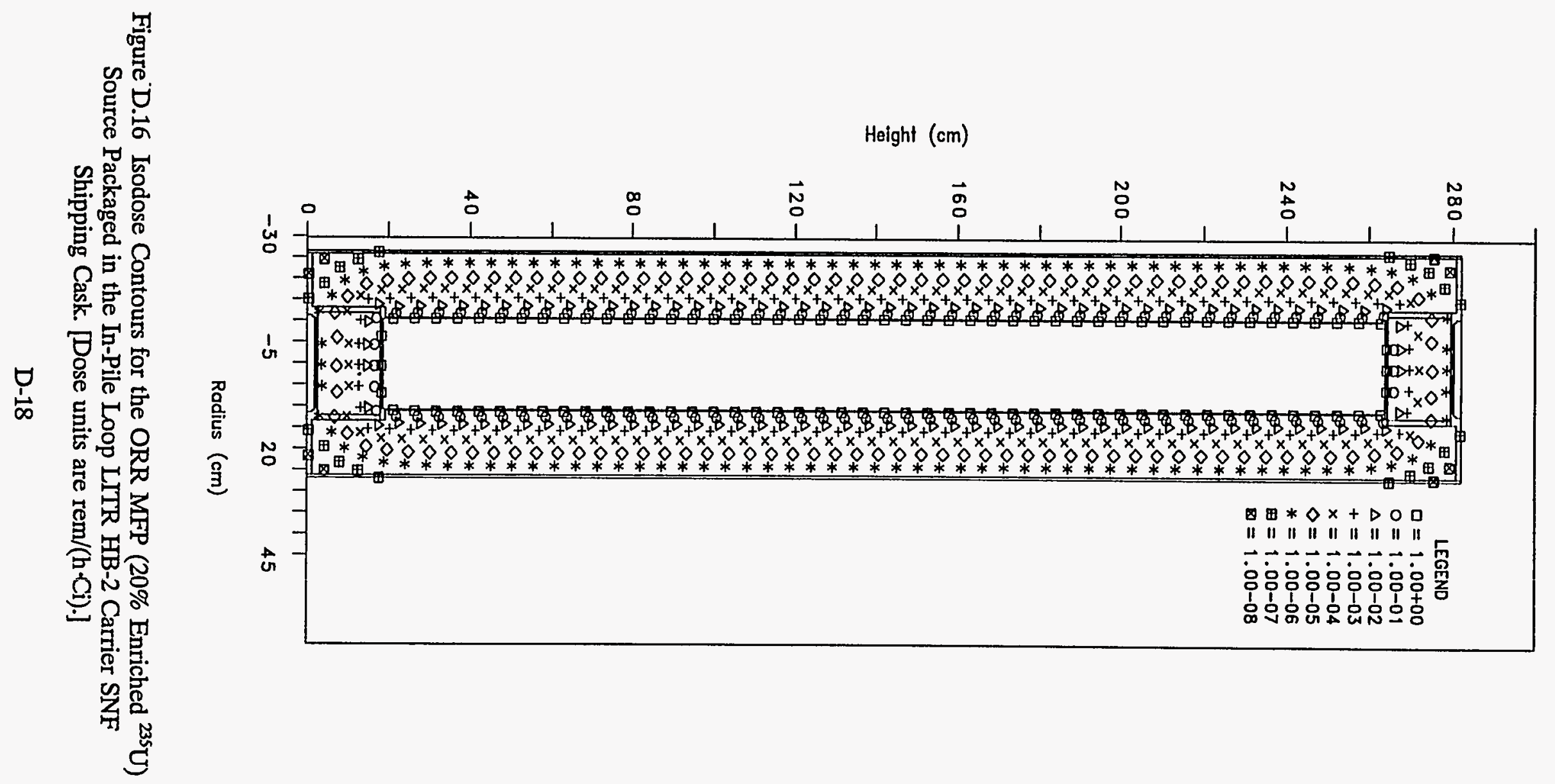




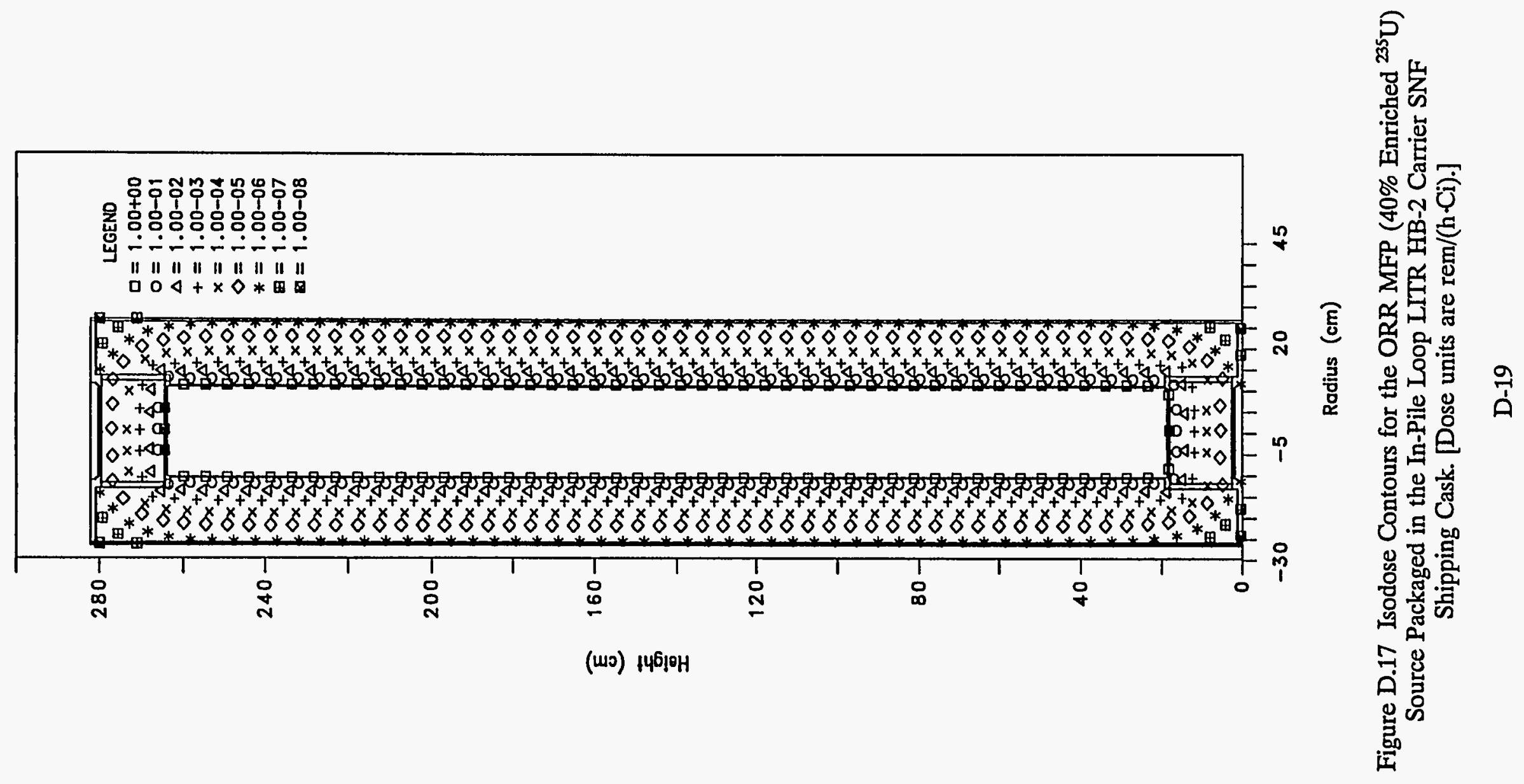




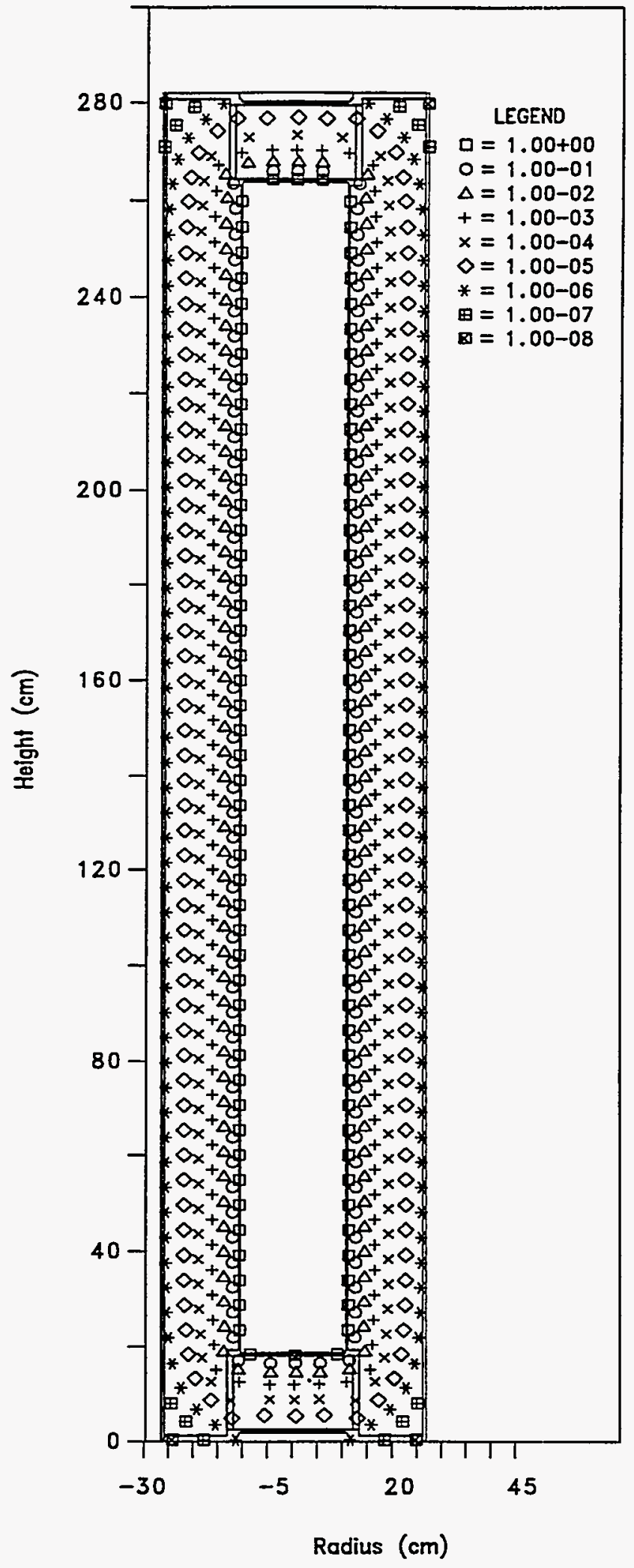

Figure D.18 Isodose Contours for the ORR MFP (93\% Enriched $\left.{ }^{235} \mathrm{U}\right)$ Source Packaged in the In-Pile Loop LITR HB-2 Carrier SNF Shipping Cask. [Dose units are rem/(h/Ci).] 


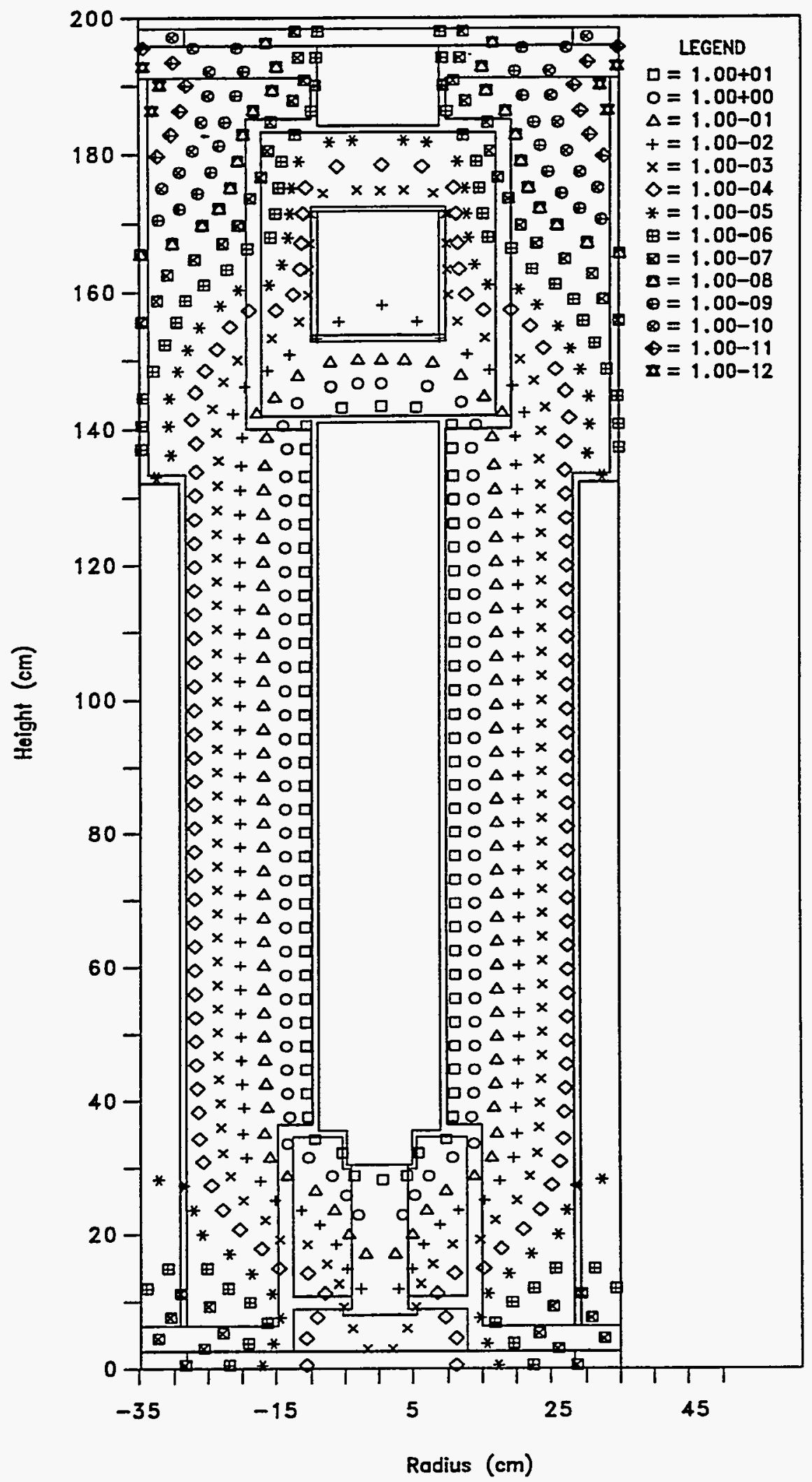

Figure D.19 Isodose Contours for the ${ }^{60} \mathrm{Co}$ Source Packaged in the 6.5-Inch HRLEL Carrier SNF Shipping Cask.

[Dose units are rem/(h-Ci).] 


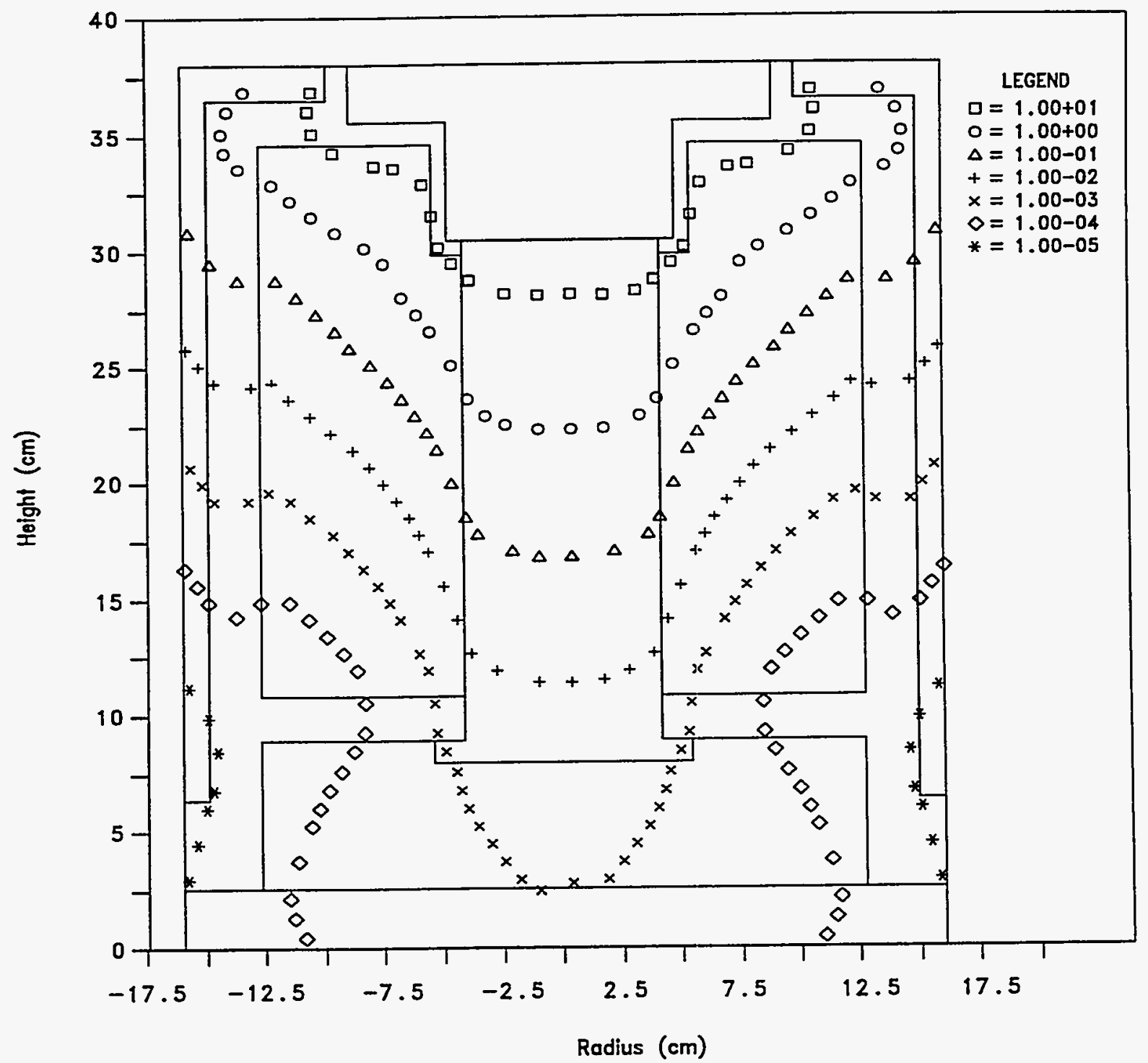

Figure D.20 Expanded View of the End Plug Isodose Contours for the ${ }^{60} \mathrm{Co}$ Source Packaged in the 6.5-Inch HRLEL Carrier SNF Shipping Cask.

[Dose units are rem/(h-Ci).]

D-22 


\section{$\varepsilon Z-\widetilde{a}$}

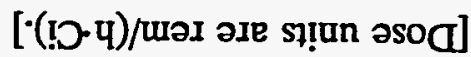

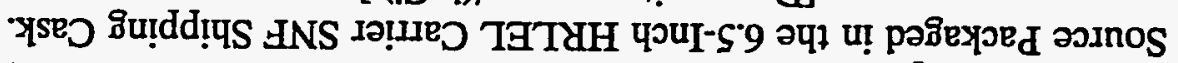

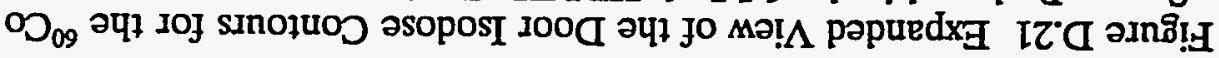

(wo) sn!pDy

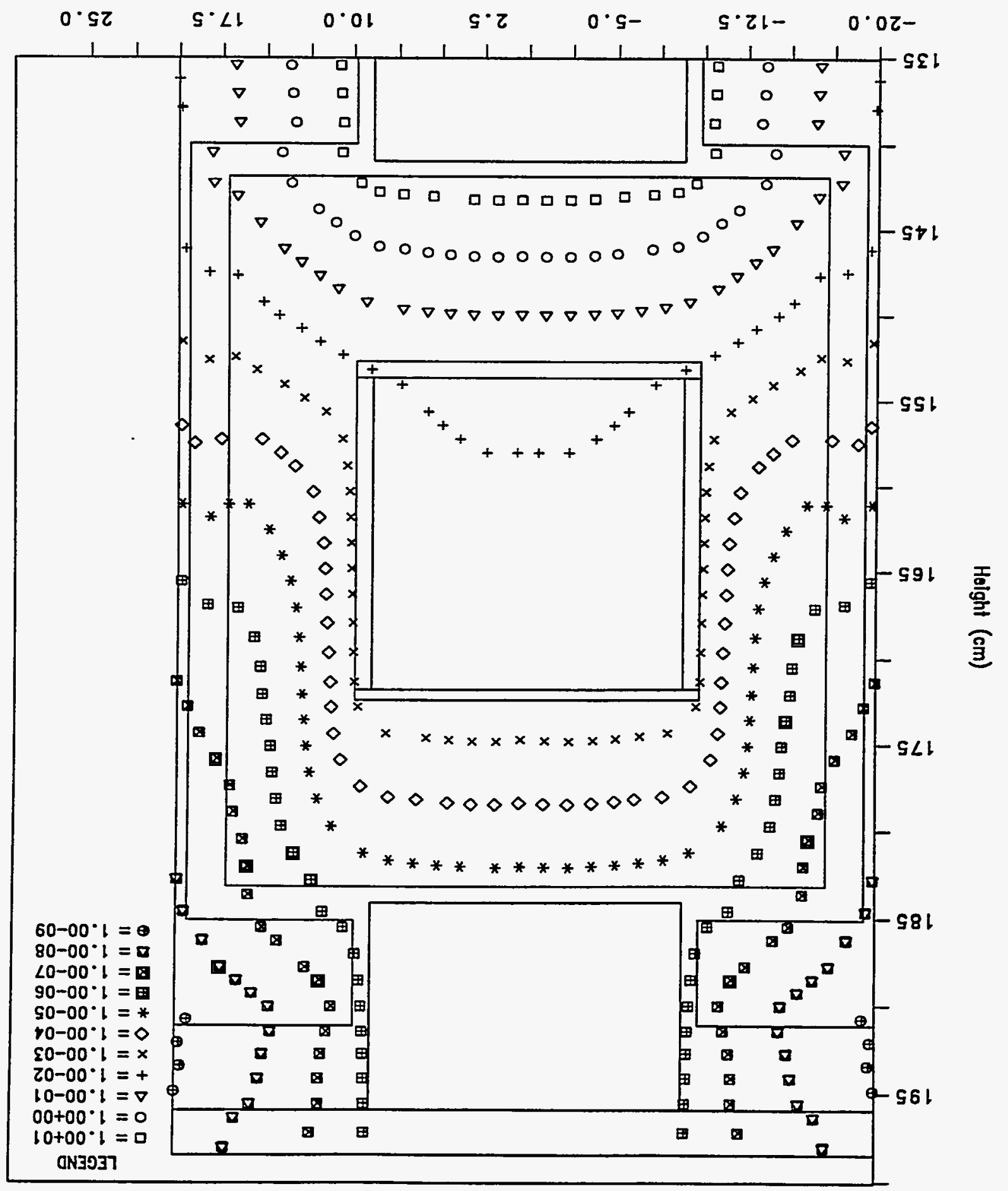


Hoight $(\mathrm{cm})$

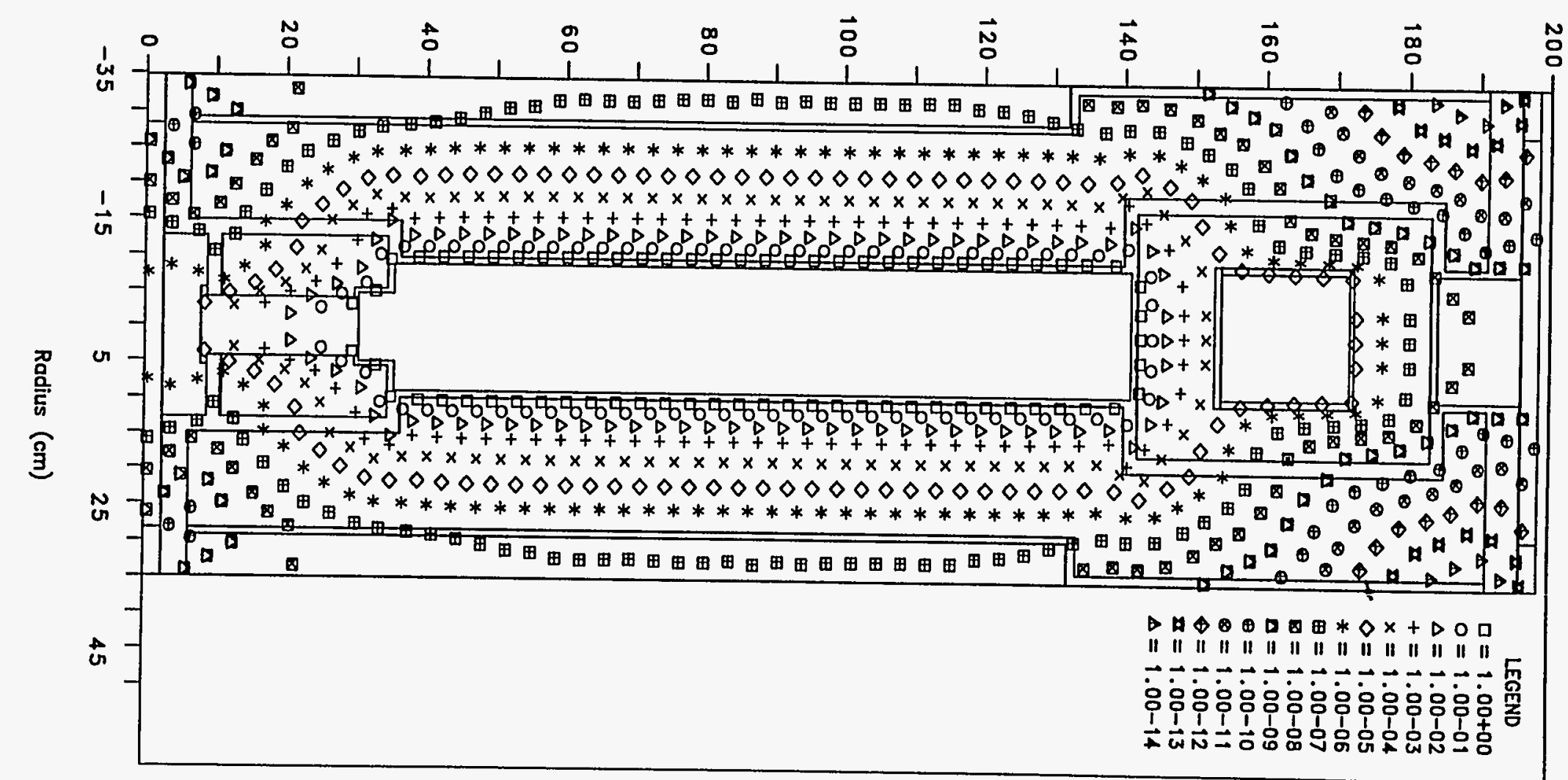




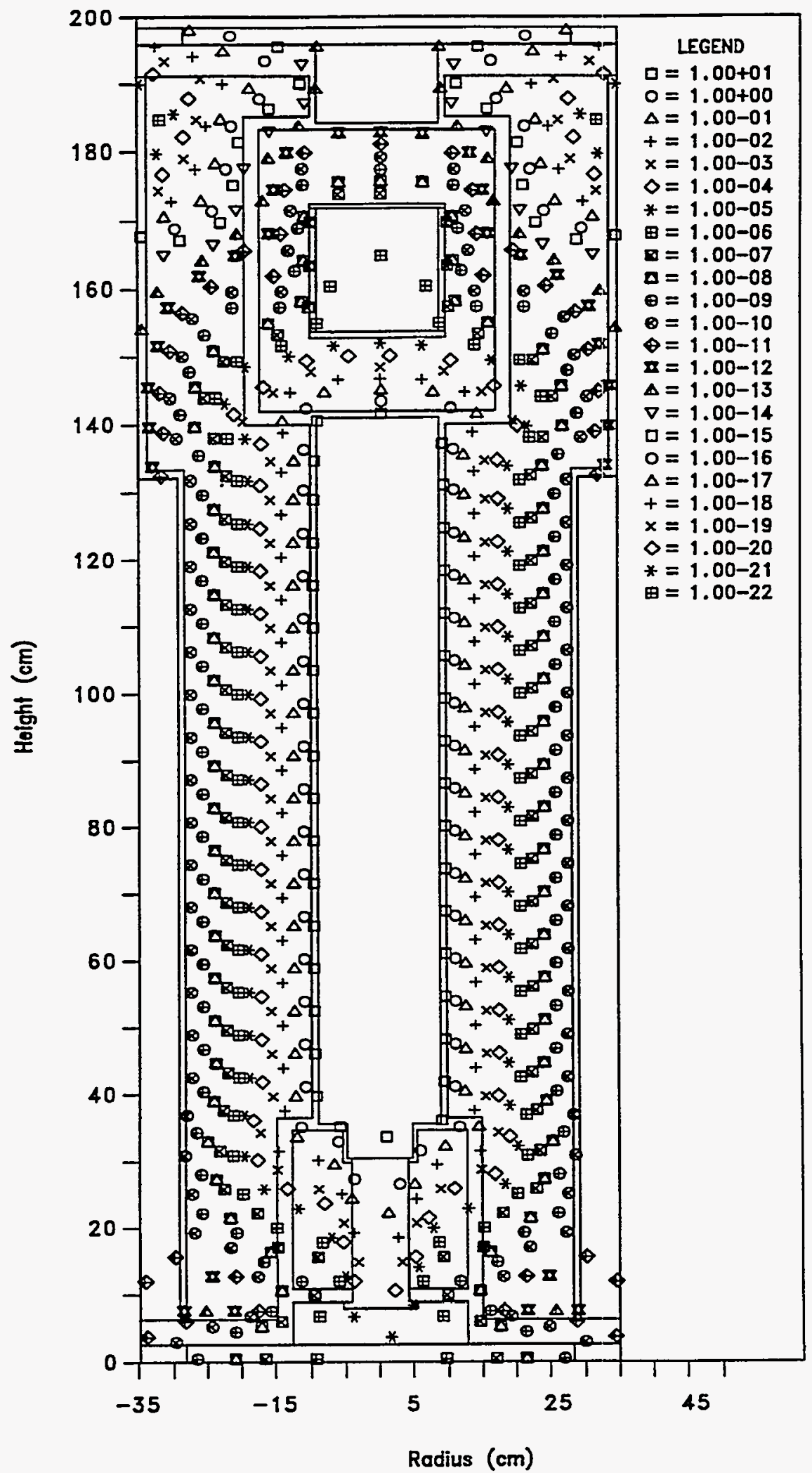

Figure D.24 Isodose Contours for the ${ }^{137} \mathrm{Cs}$ Source Packaged in the 6.5-Inch HRLEL Carrier SNF Shipping Cask.

[Dose units are rem/(h-Ci).]

D-26 


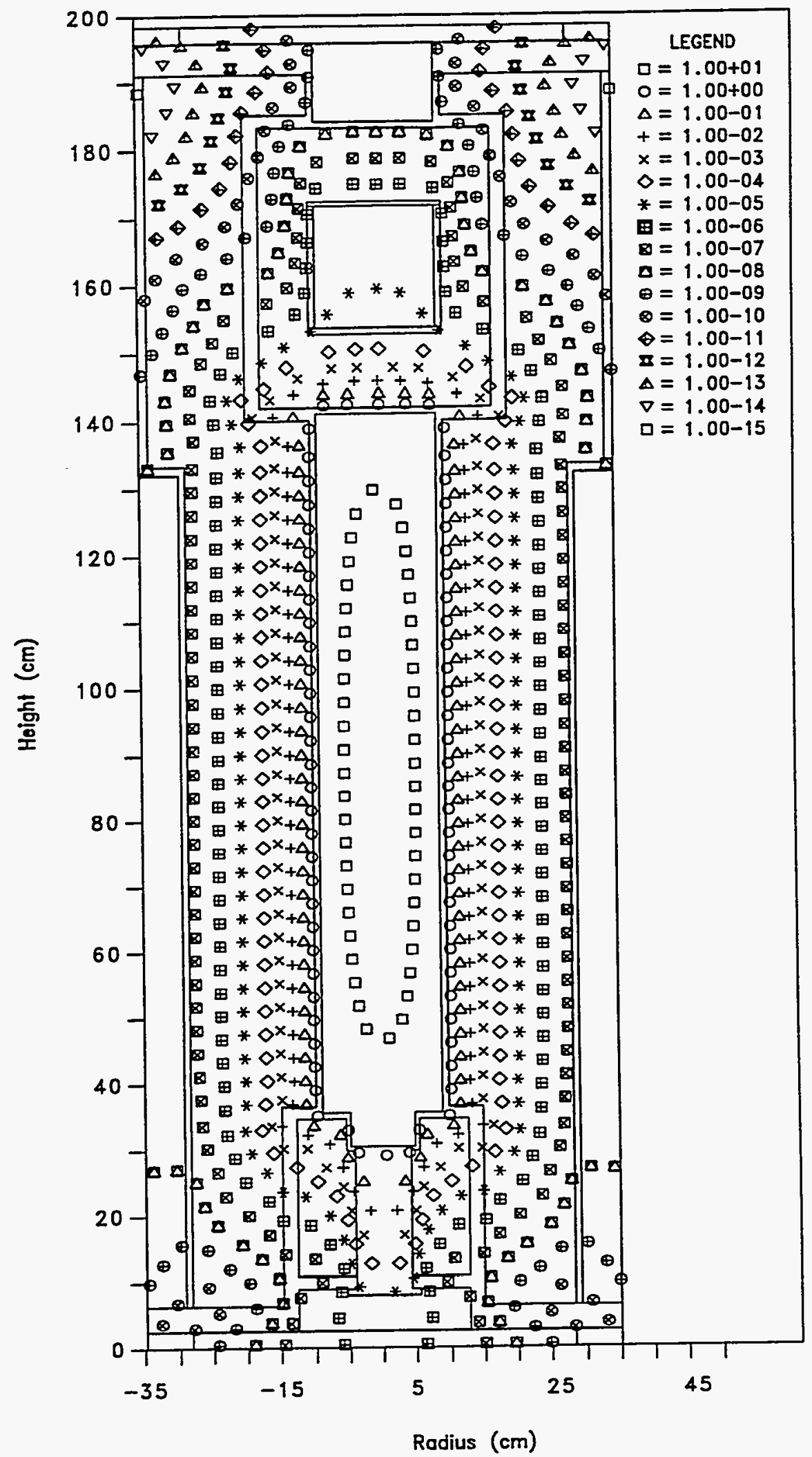

Figure D.25 Isodose Contours for the ORR MFP (20\% Enriched ${ }^{235} \mathrm{U}$ ) Source Packaged in the 6.5-Inch HRLEL Carrier SNF Shipping Cask. [Dose units are rem/(h.Ci).] 


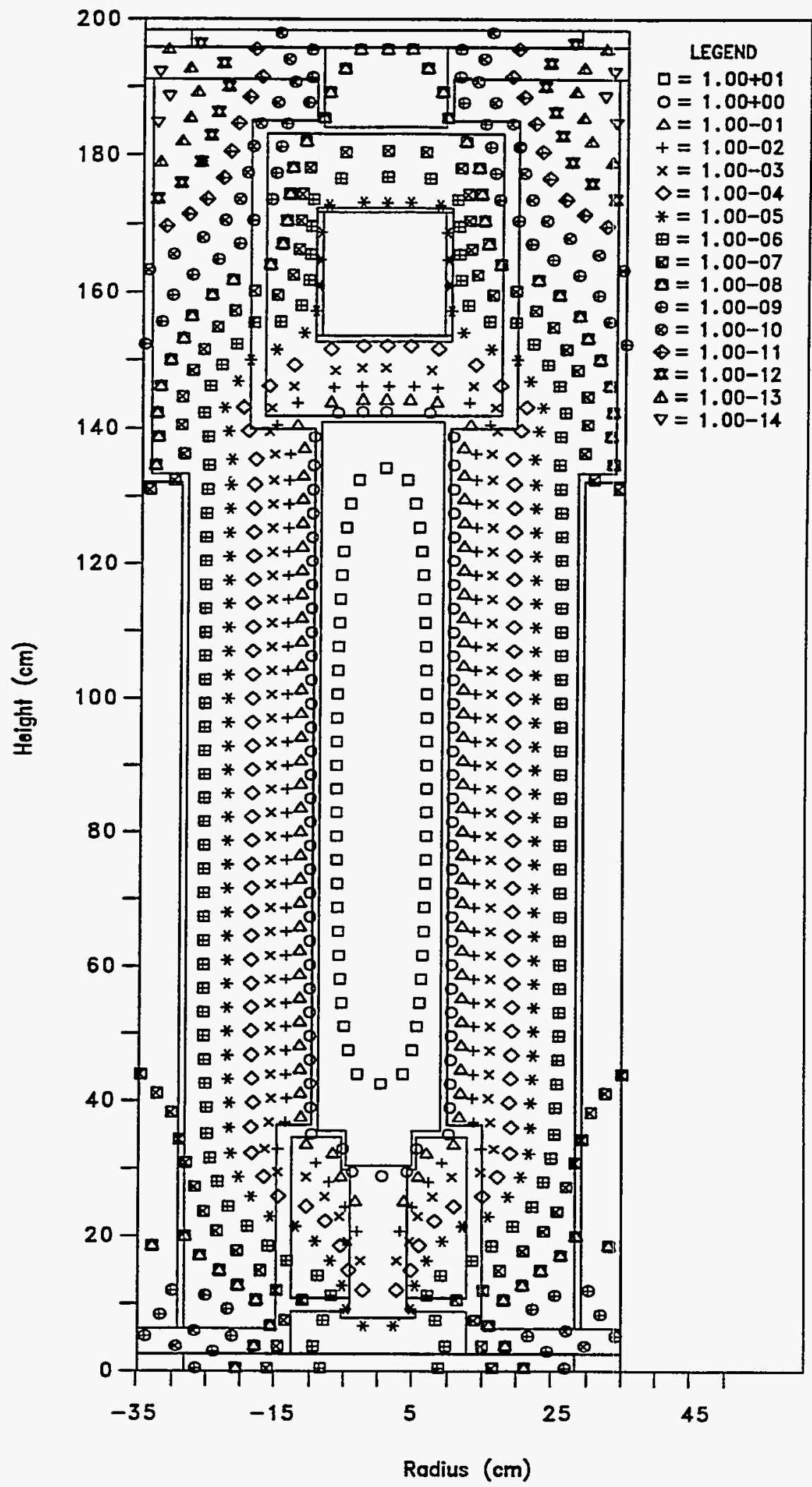

Figure D.26 Isodose Contours for the ORR MFP (40\% Enriched ${ }^{235} \mathrm{U}$ ) Source Packaged in the 6.5-Inch HRLEL Carrier SNF Shipping Cask.

[Dose units are rem/(h-Ci).] 


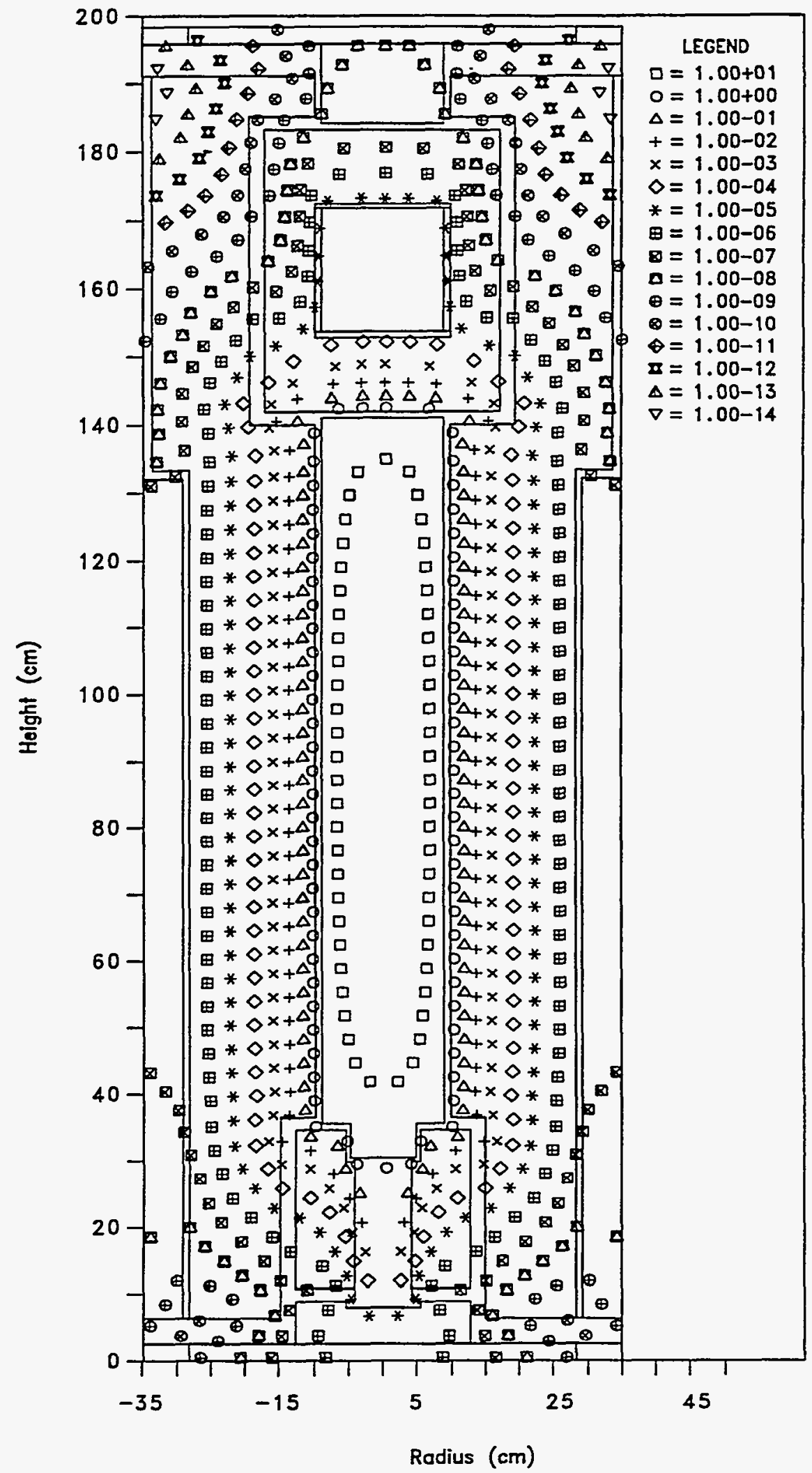

Figure D.27 Isodose Contours for the ORR MFP (93\% Enriched ${ }^{235} \mathrm{U}$ ) Source Packaged in the 6.5-Inch HRLEL Carrier SNF Shipping Cask.

[Dose units are rem/(h-Ci).] 


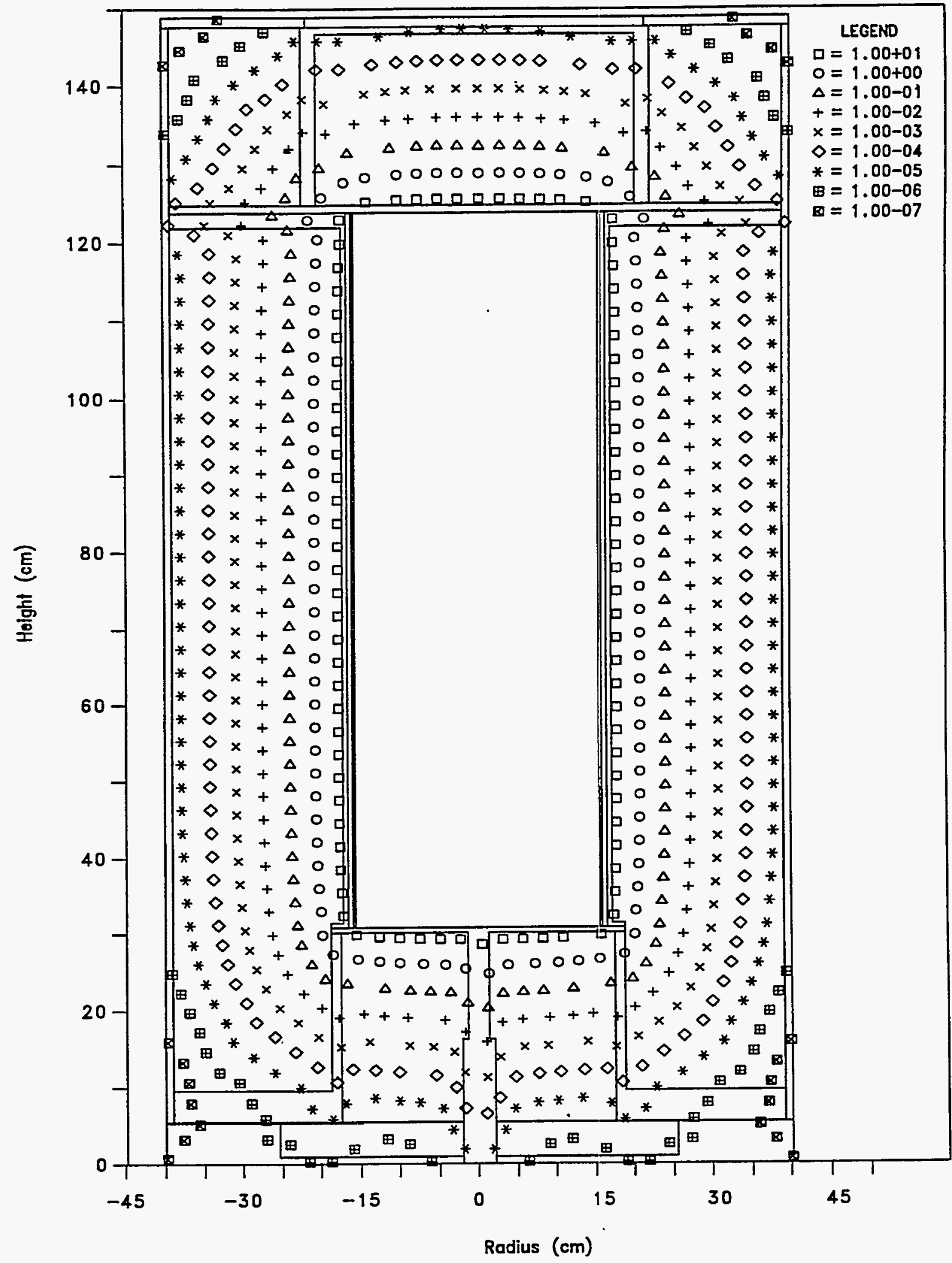

Figure D.28 Isodose Contours for the ${ }^{60} \mathrm{Co}$ Source Packaged in the HFIR Hot Scrap Carrier SNF Shipping Cask.

[Dose units are rem/(h-Ci).] 


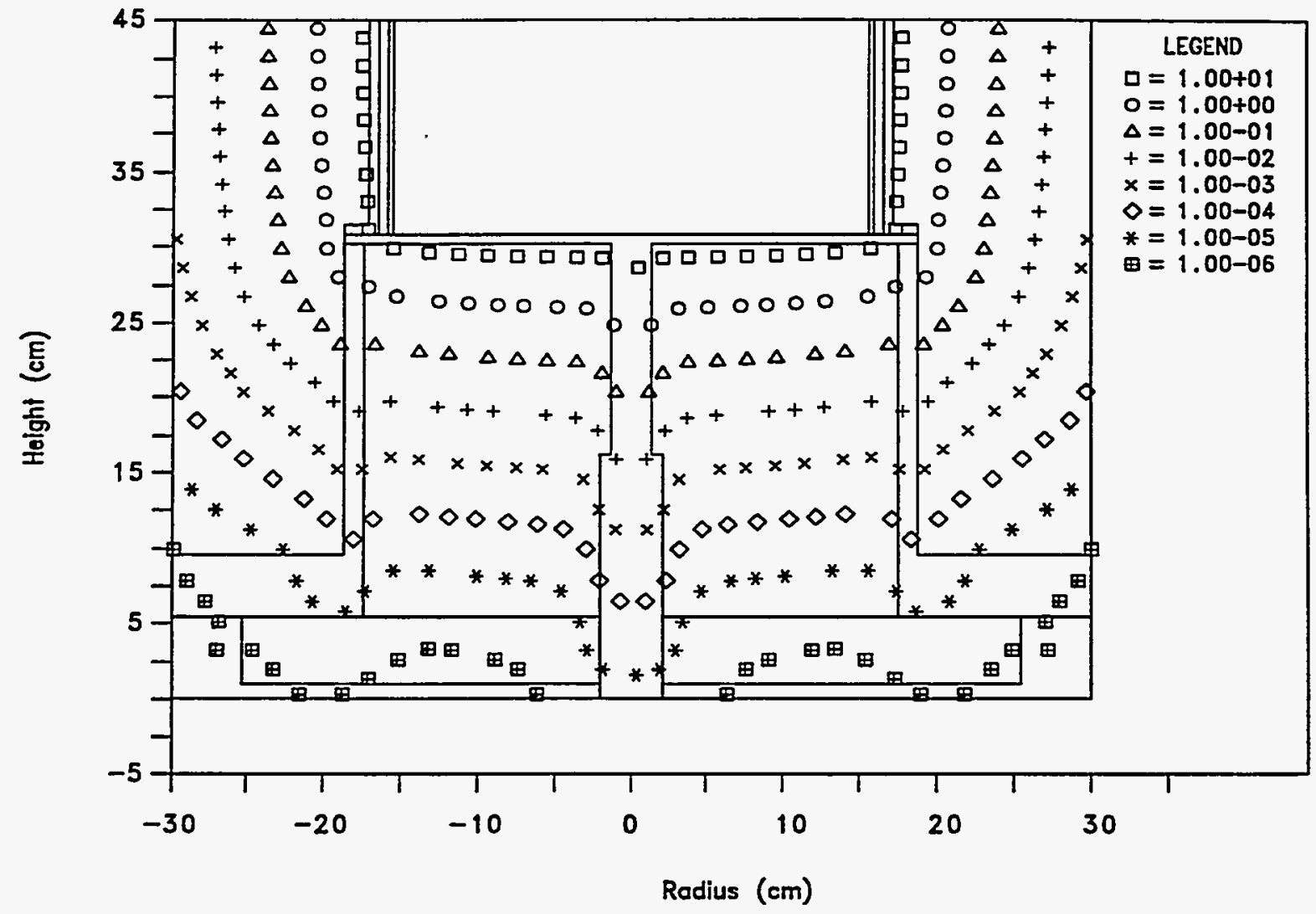

Figure D.29 Expanded View of the Top Plug Isodose Contours for the ${ }^{60} \mathrm{Co}$ Source Packaged in the HFIR Hot Scrap Carrier SNF

Shipping Cask. [Dose units are rem/(h.Ci).] 


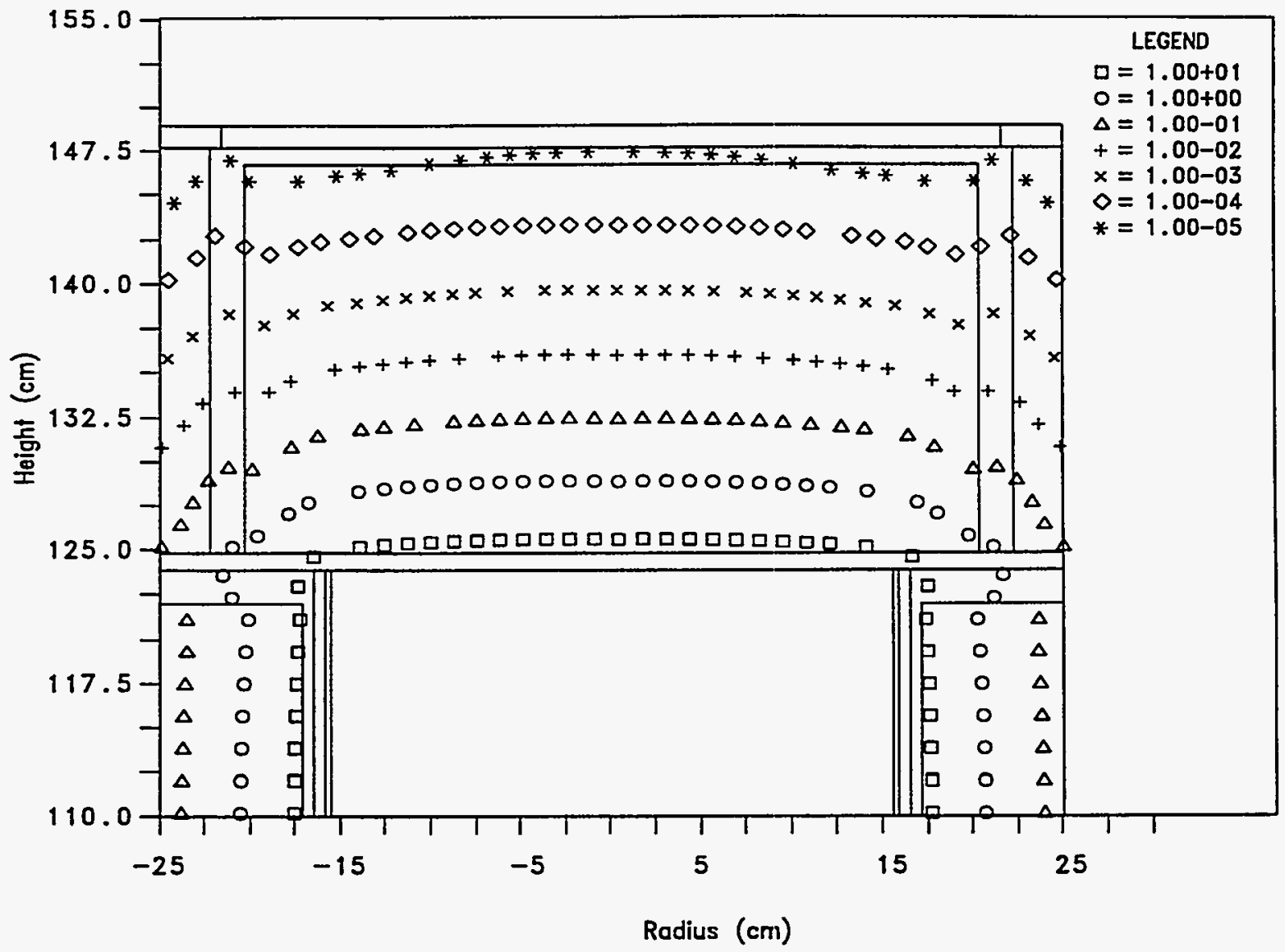

Figure D.30 Expanded View of the Bottom Door Isodose Contours for the ${ }^{60}$ Co Source Packaged in the HFIR Hot Scrap Carrier SNF Shipping Cask.

[Dose units are rem/(h-Ci).] 


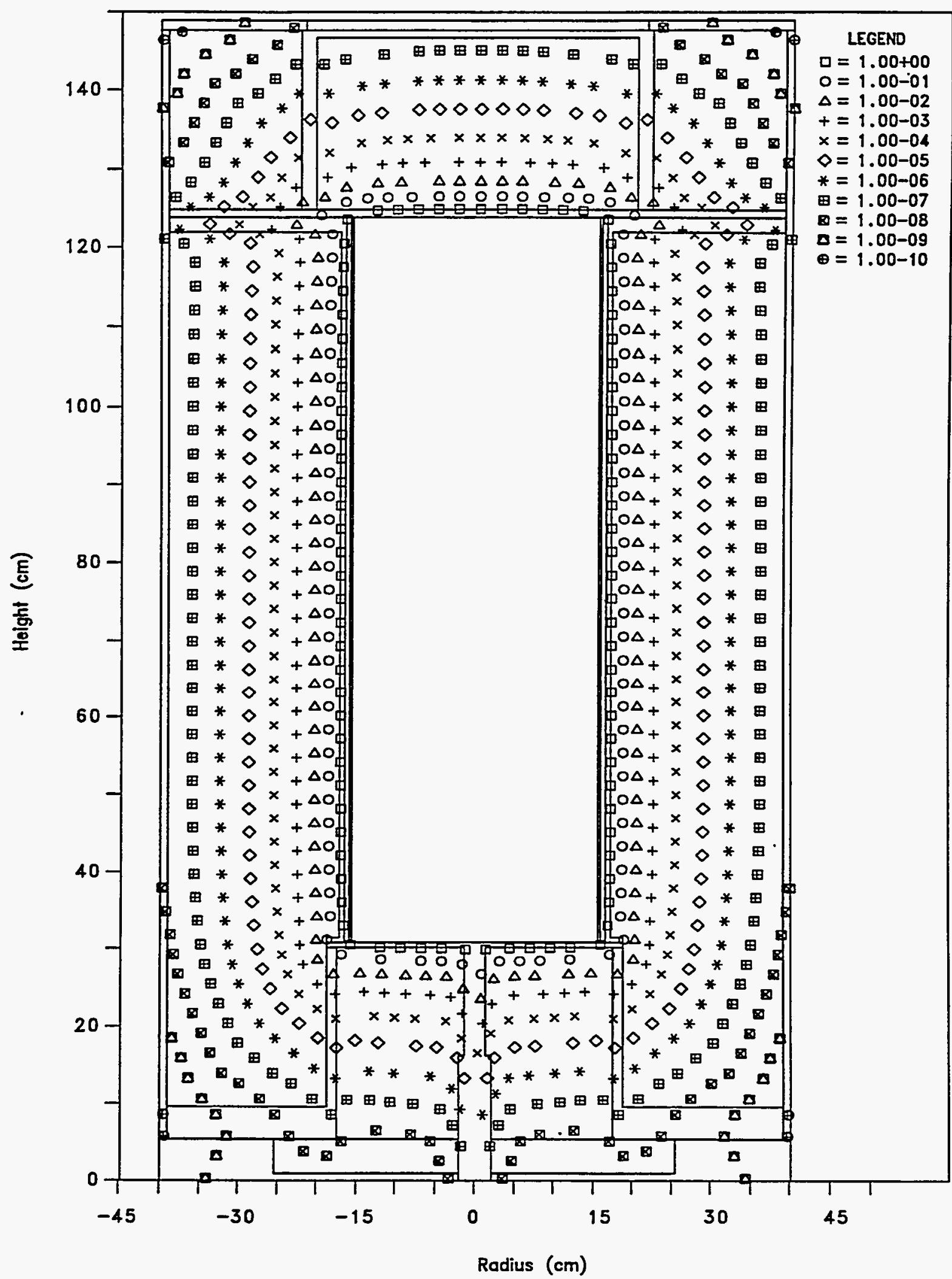

Figure D.31 Isodose Contours for the LWR MFP (25 MWd/kgU) Source Packaged in the HFIR Hot Scrap Carrier SNF Shipping Cask. [Dose units are $\mathrm{rem} /(\mathrm{h} \cdot \mathrm{Ci})$.] 


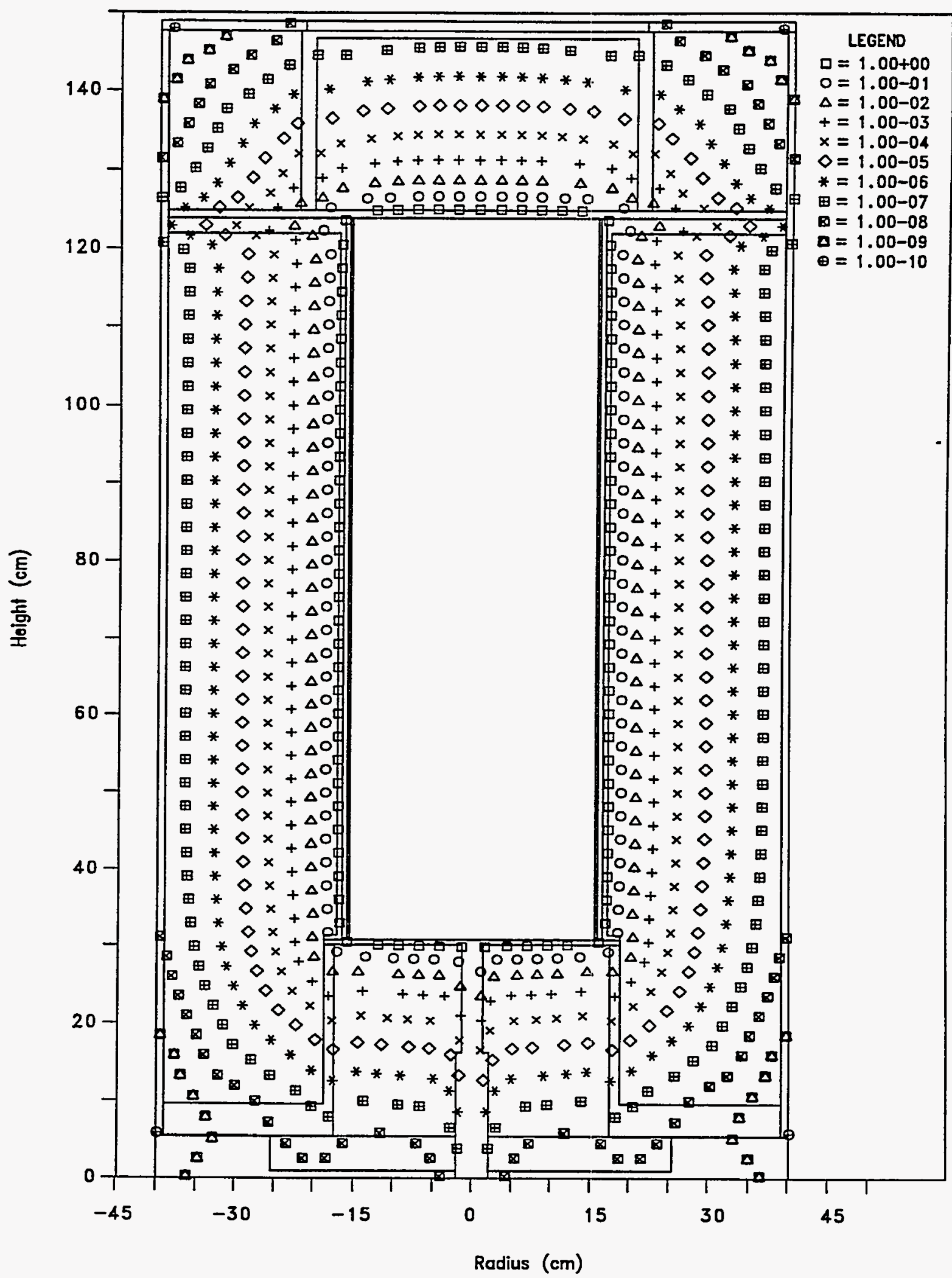

Figure D.32 Isodose Contours for the LWR MFP (50 MWd/kgU) Source Packaged in the HFIR Hot Scrap Carrier SNF Shipping Cask.

[Dose units are $\mathrm{rem} /(\mathrm{h} \cdot \mathrm{Ci})$.] 


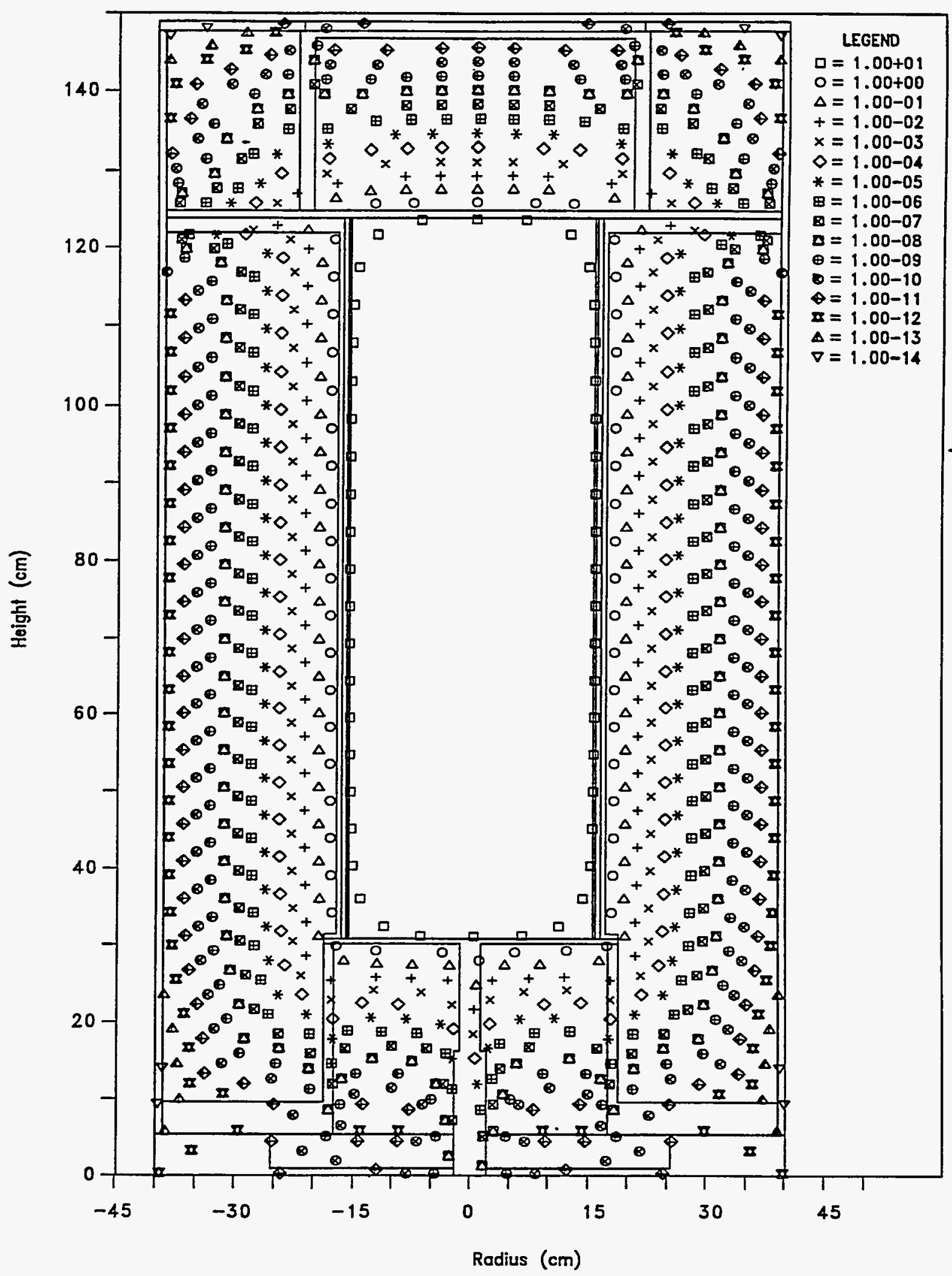

Figure D.33 Isodose Contours for the ${ }^{137} \mathrm{Cs}$ Source Packaged in the HFIR Hot Scrap Carrier SNF Shipping Cask.

[Dose units are rem/(h.Ci).] 


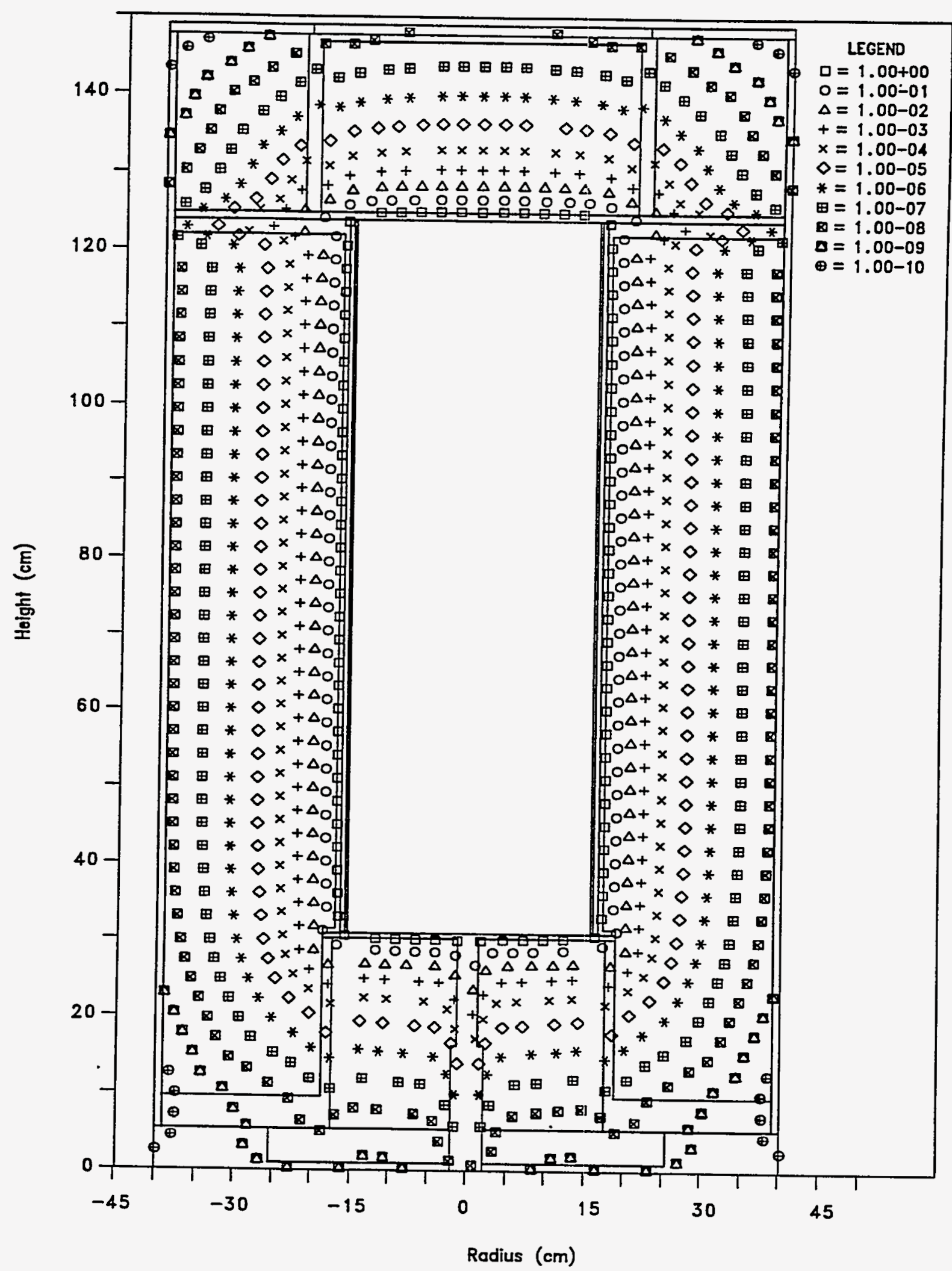

Figure D.34 Isodose Contours for the ORR MFP (20\% Enriched ${ }^{235} \mathrm{U}$ ) Source Packaged in the HFIR Hot Scrap Carrier SNF Shipping Cask.

[Dose units are rem/(h-Ci).] 


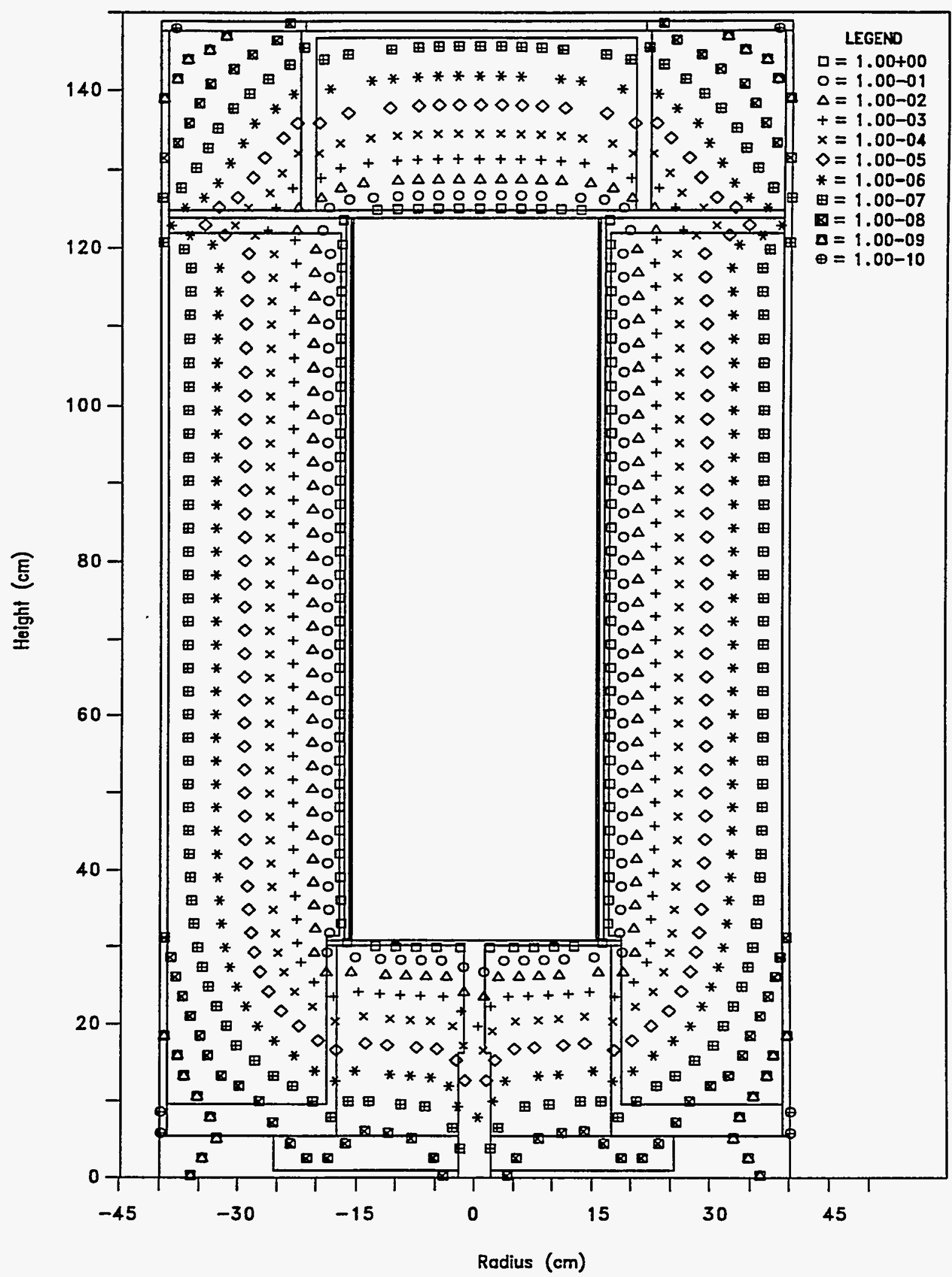

Figure D.35 Isodose Contours for the ORR MFP (40\% Enriched ${ }^{235} \mathrm{U}$ ) Source Packaged in the HFIR Hot Scrap Carrier SNF Shipping Cask.

[Dose units are $\mathrm{rem} /(\mathrm{h} \cdot \mathrm{Ci})$.] 


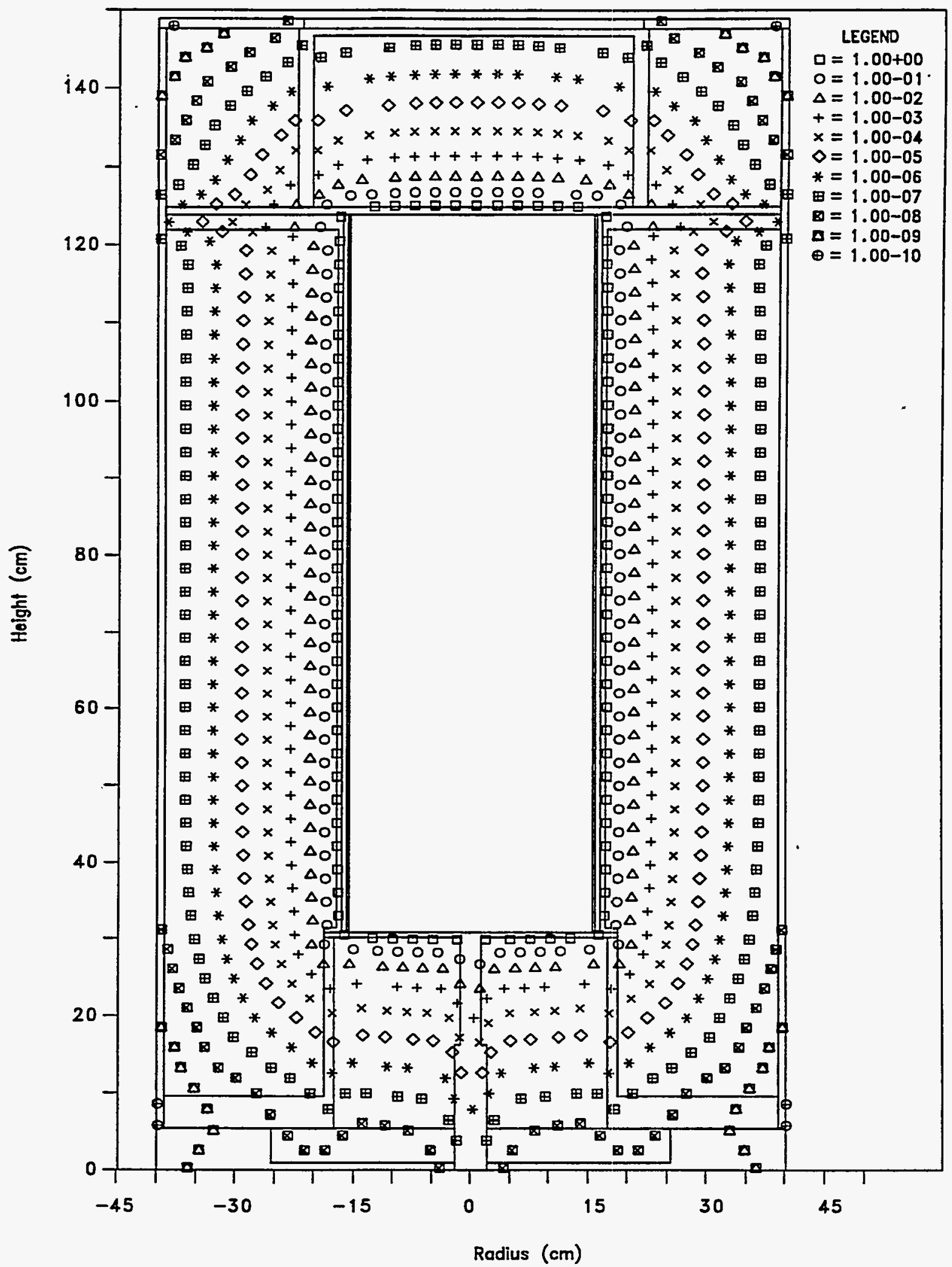

Figure D.36 Isodose Contours for the ORR MFP (93\% Enriched ${ }^{235} \mathrm{U}$ ) Source Packaged in the HFIR Hot Scrap Carrier SNF Shipping Cask.

[Dose units are rem/(h-Ci).] 


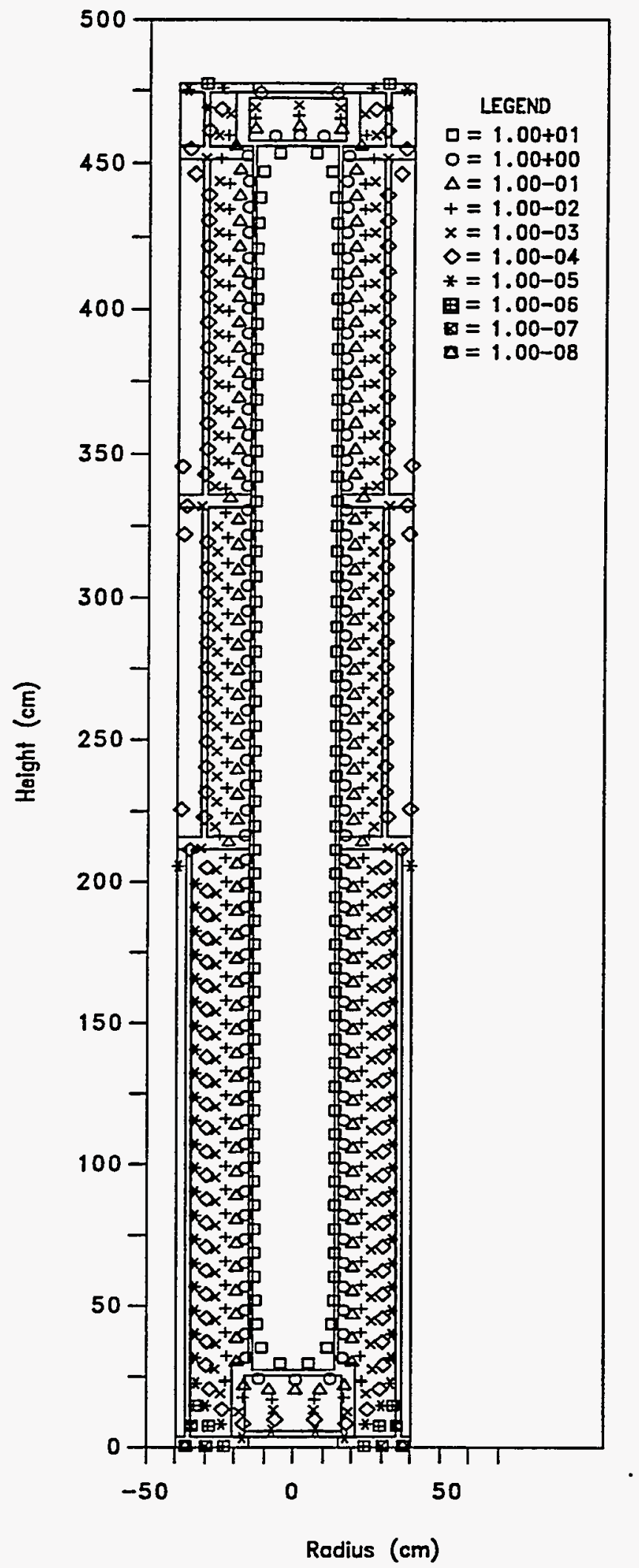

Figure D.37 Isodose Contours for the ${ }^{60} \mathrm{Co}$ Source Packaged in the 10-Inch ORR Experiment Removal Shield SNF Shipping Cask. [Dose units are $\mathrm{rem} /(\mathrm{h} \cdot \mathrm{Ci})$.]

D-39 


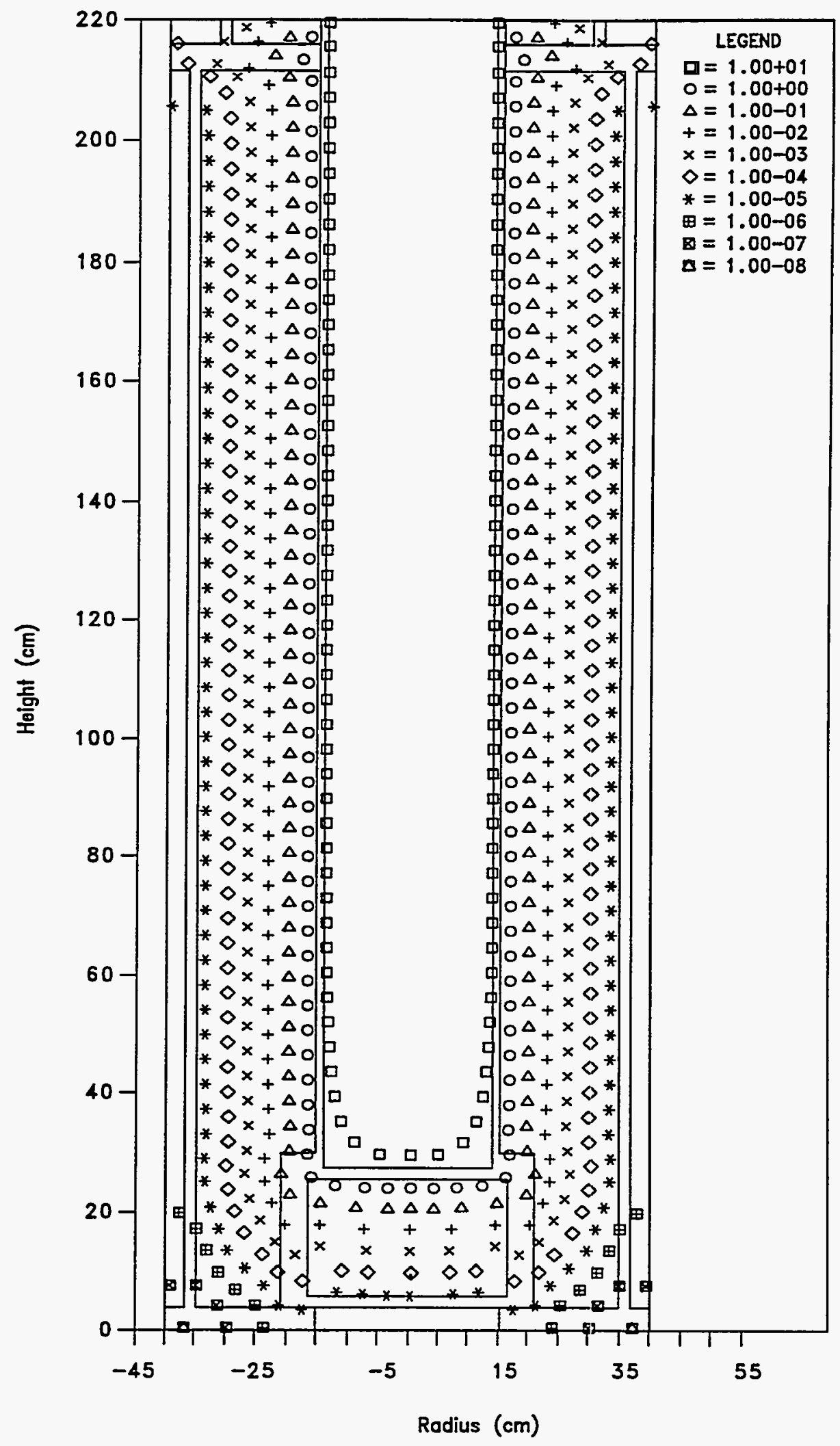

Figure D-38. Expanded View of the Heavy Shield Section Isodose Contours for the ${ }^{60} \mathrm{Co}$ Source Packaged in the 10-Inch ORR Experiment Removal Shield SNF Shipping Cask. [Dose units are rem/(h.Ci).] 


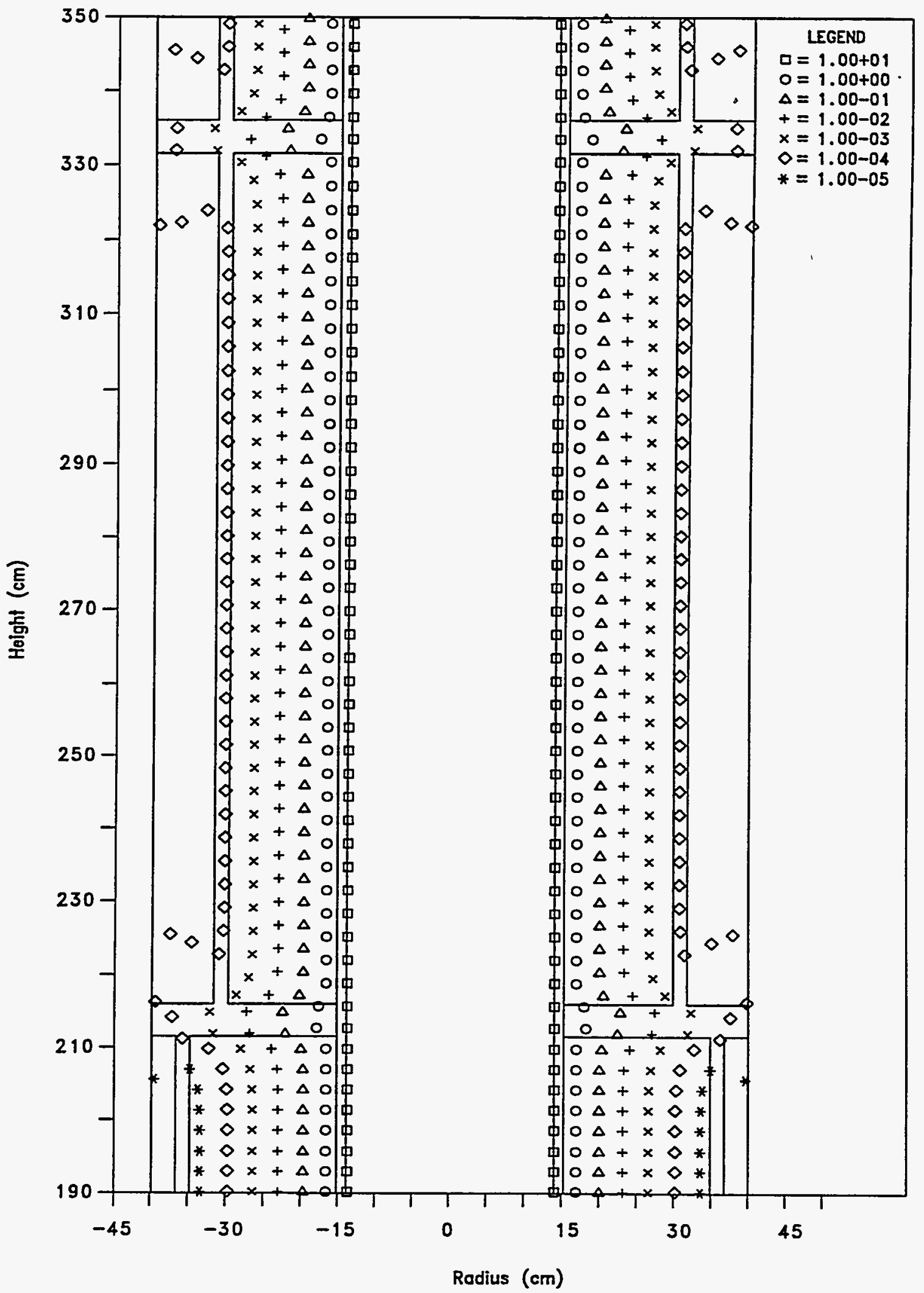

Figure D.39 Expanded View of the Central Light Shield Section Isodose Contours for the ${ }^{60} \mathrm{Co}$ Source Packaged in the 10-Inch ORR Experiment

Removal Shield SNF Shipping Cask. [Dose units are rem/(h-Ci).] 


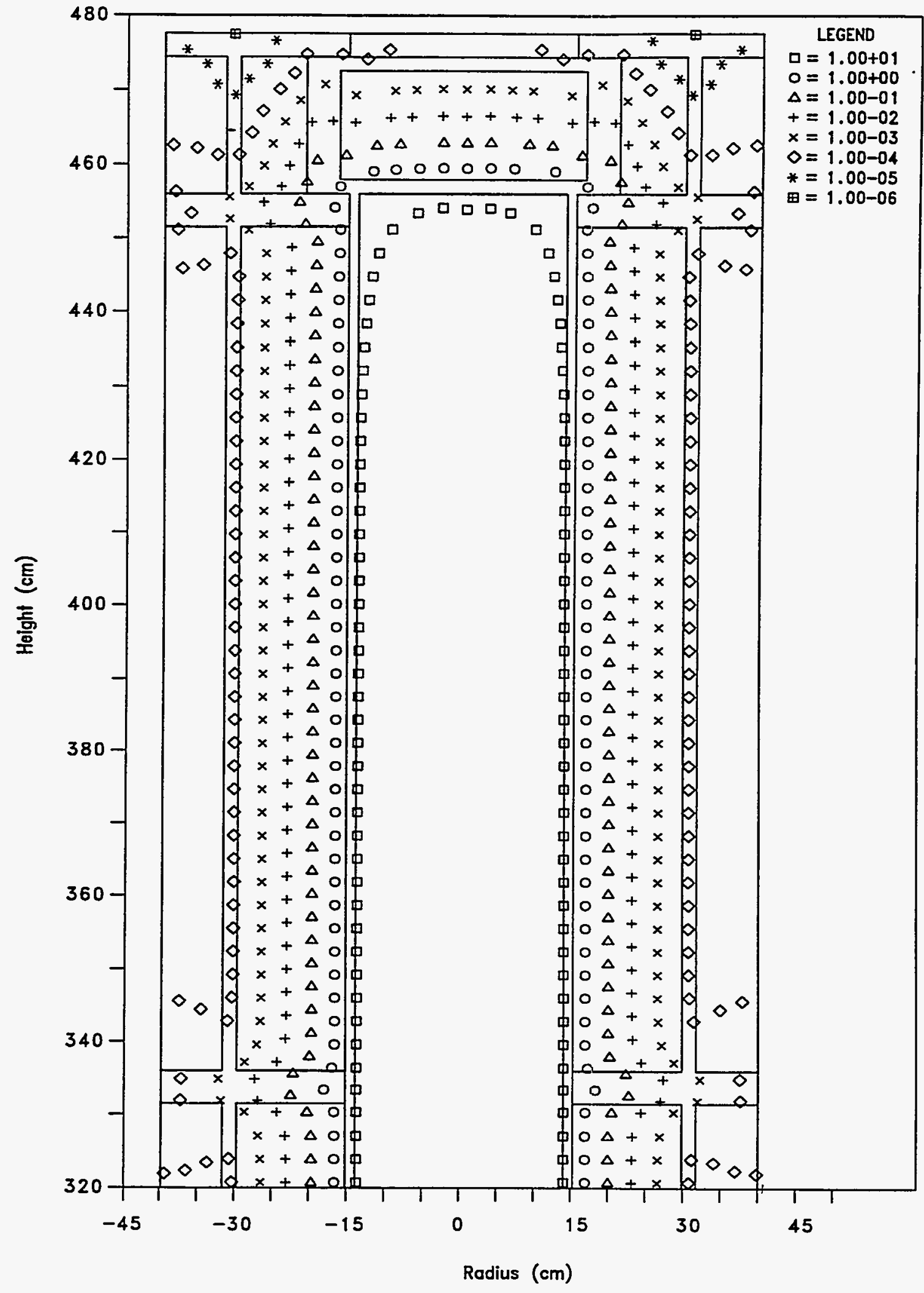

Figure D.40 Expanded View of the Light Shield Section Isodose Contours for the ${ }^{60} \mathrm{Co}$ Source Packaged in the 10-Inch ORR Experiment Removal Shield SNF Shipping Cask. [Dose units are rem/h-Ci).] 


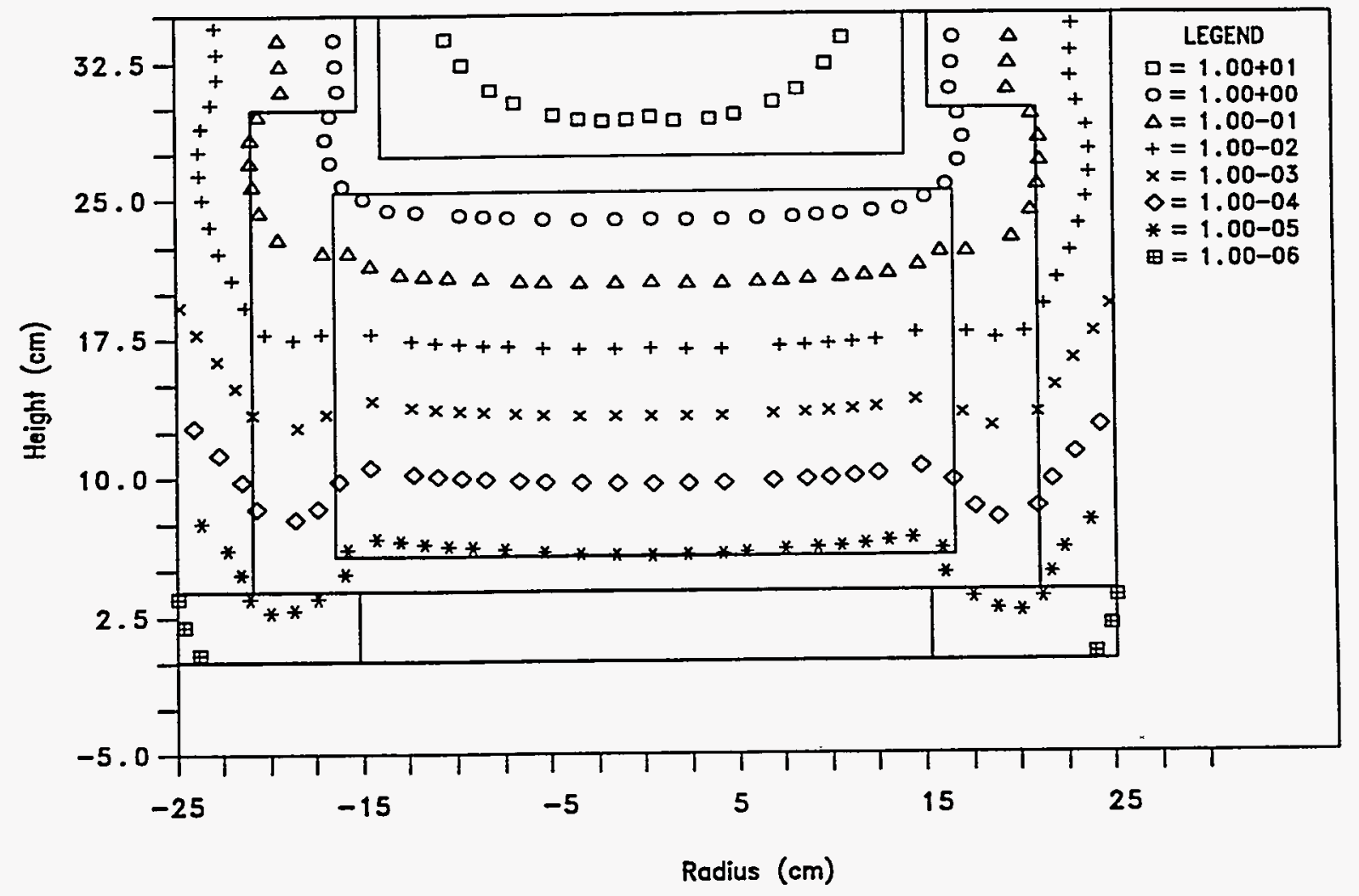

Figure D.41 Expanded View of the Heavy Door Isodose Contours for the ${ }^{60} \mathrm{Co}$ Source Packaged in the 10-Inch ORR Experiment Removal Shield SNF Shipping Cask. [Dose units are $\mathrm{rem} /(\mathrm{h} \cdot \mathrm{Ci})$.]

\section{D-43}




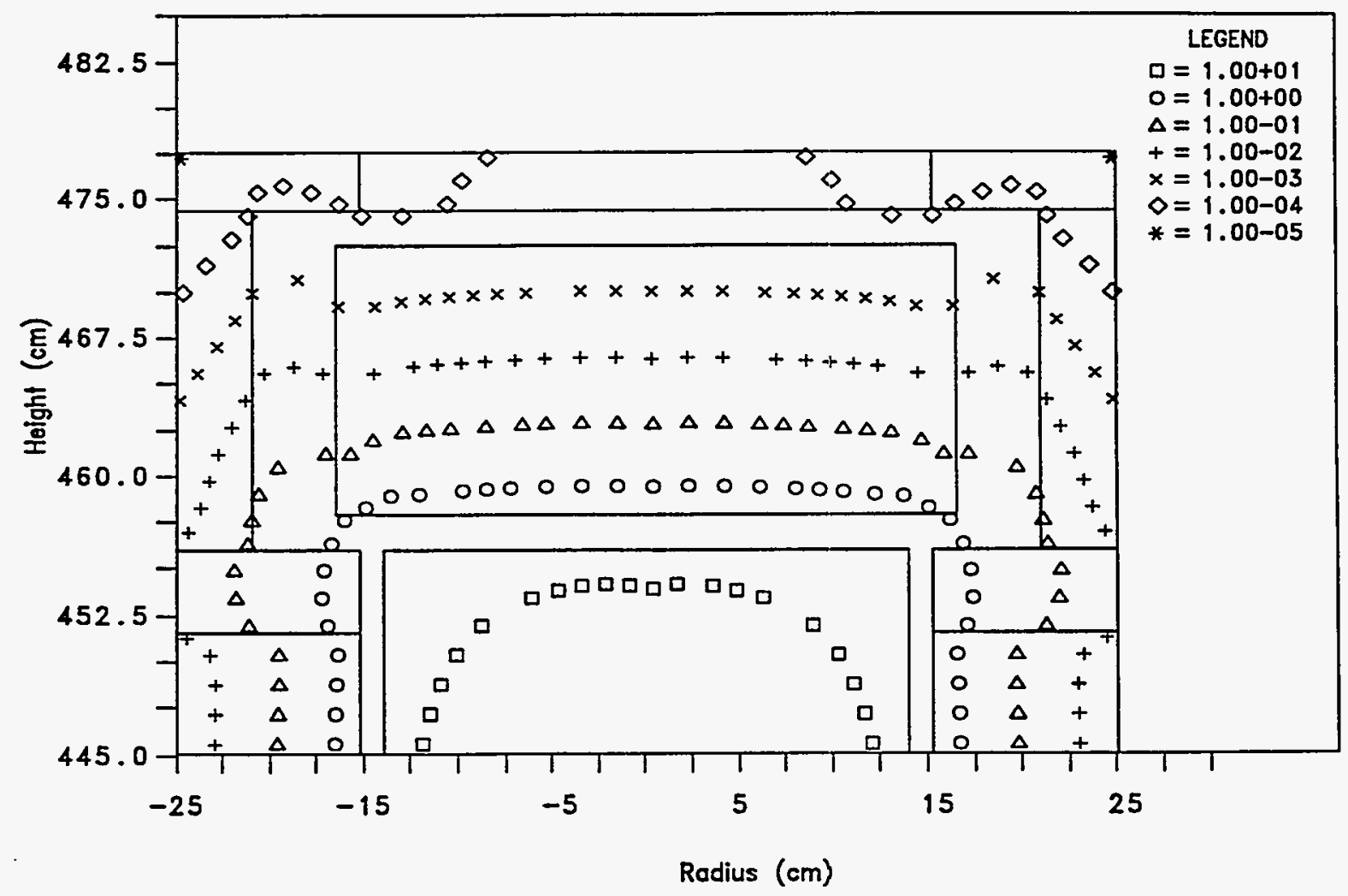

Figure D.42 Expanded View of the Light Door Isodose Contours for the ${ }^{60} \mathrm{Co}$ Source Packaged in the 10-Inch ORR Experiment Removal Shield SNF Shipping Cask. [Dose units are rem/(h.Ci).] 


\section{St- $\mathbb{a}$}

[·(เ:

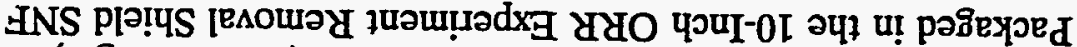

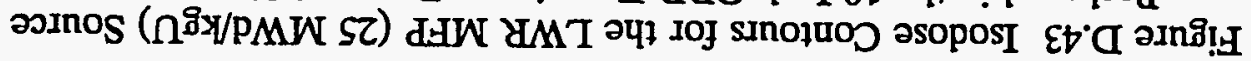

(wo) sn!poy

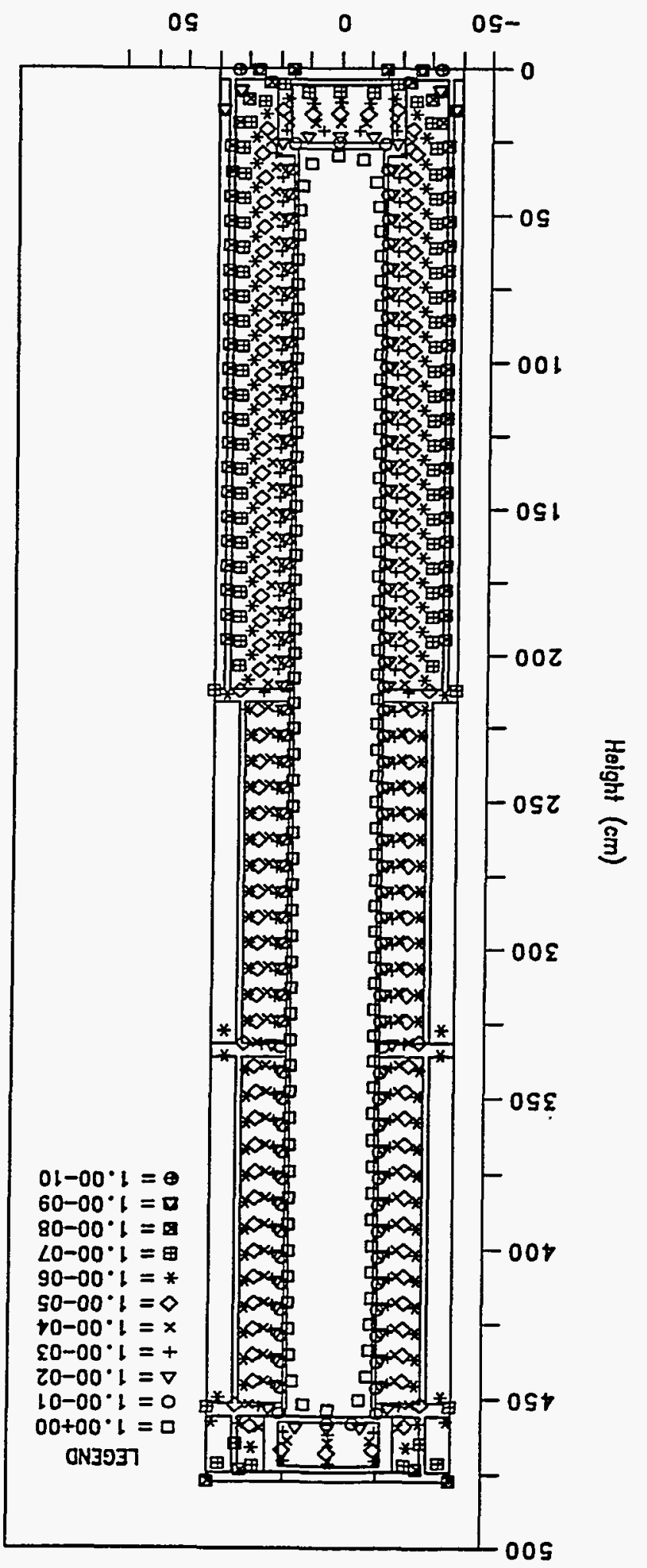




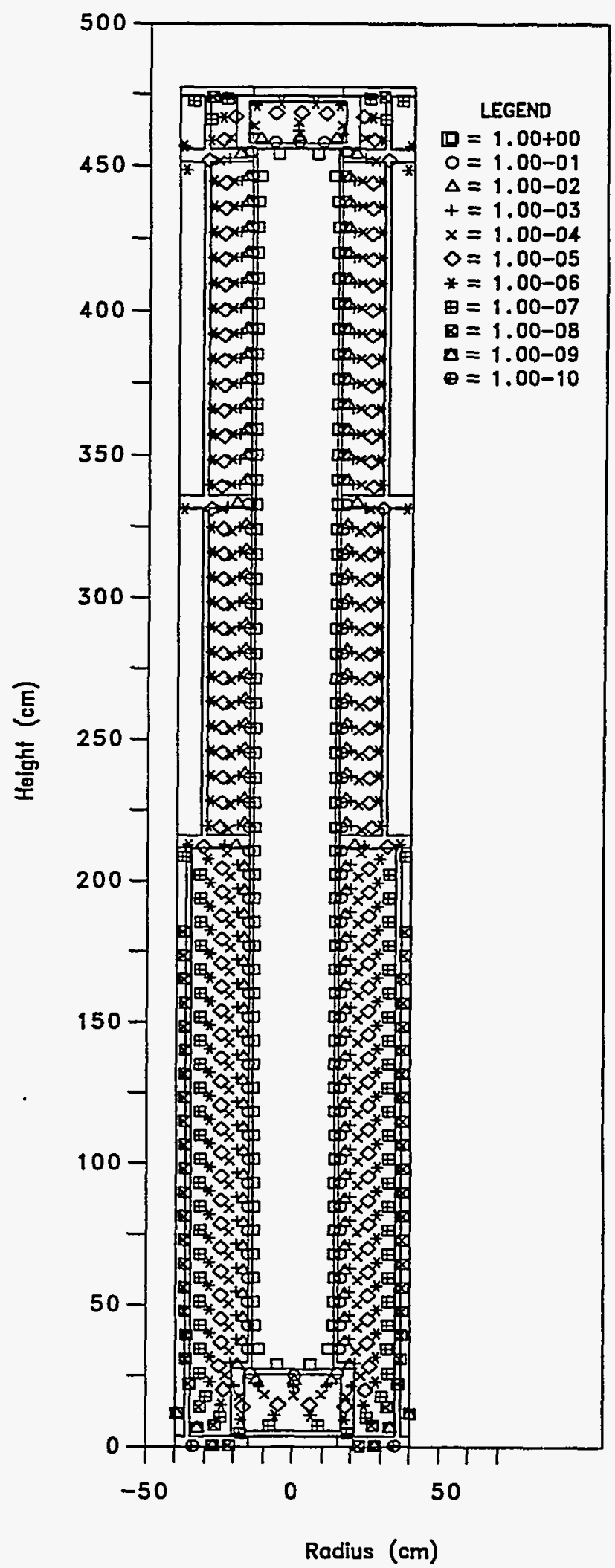

Figure D.44 Isodose Contours for the LWR MFP (50 MWd/kgU) Source Packaged in the 10-Inch ORR Experiment Removal Shield SNF Shipping Cask. [Dose units are rem/(h.Ci).] 


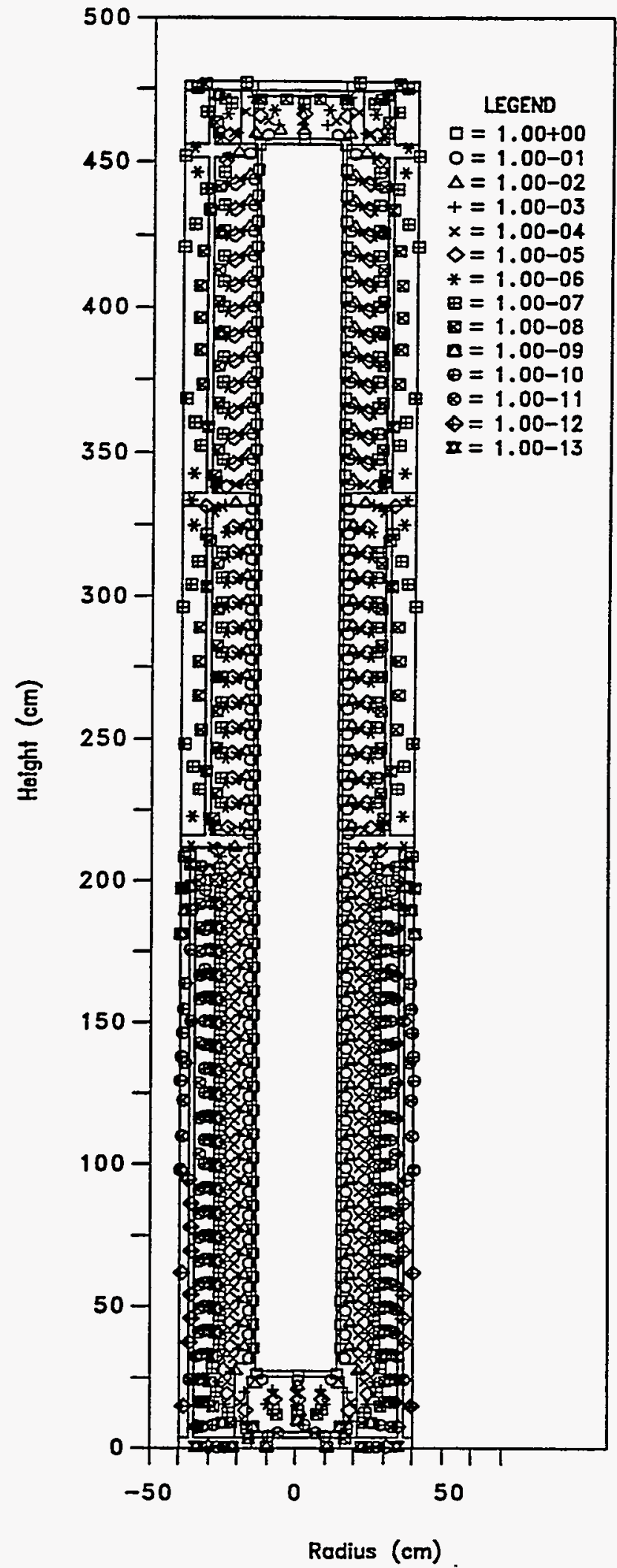

Figure D.45 Isodose Contours for the ${ }^{137} \mathrm{Cs}$ Source Packaged in the 10-Inch ORR Experiment Removal Shield SNF Shipping Cask.

[Dose units are rem/(h·Ci).] 


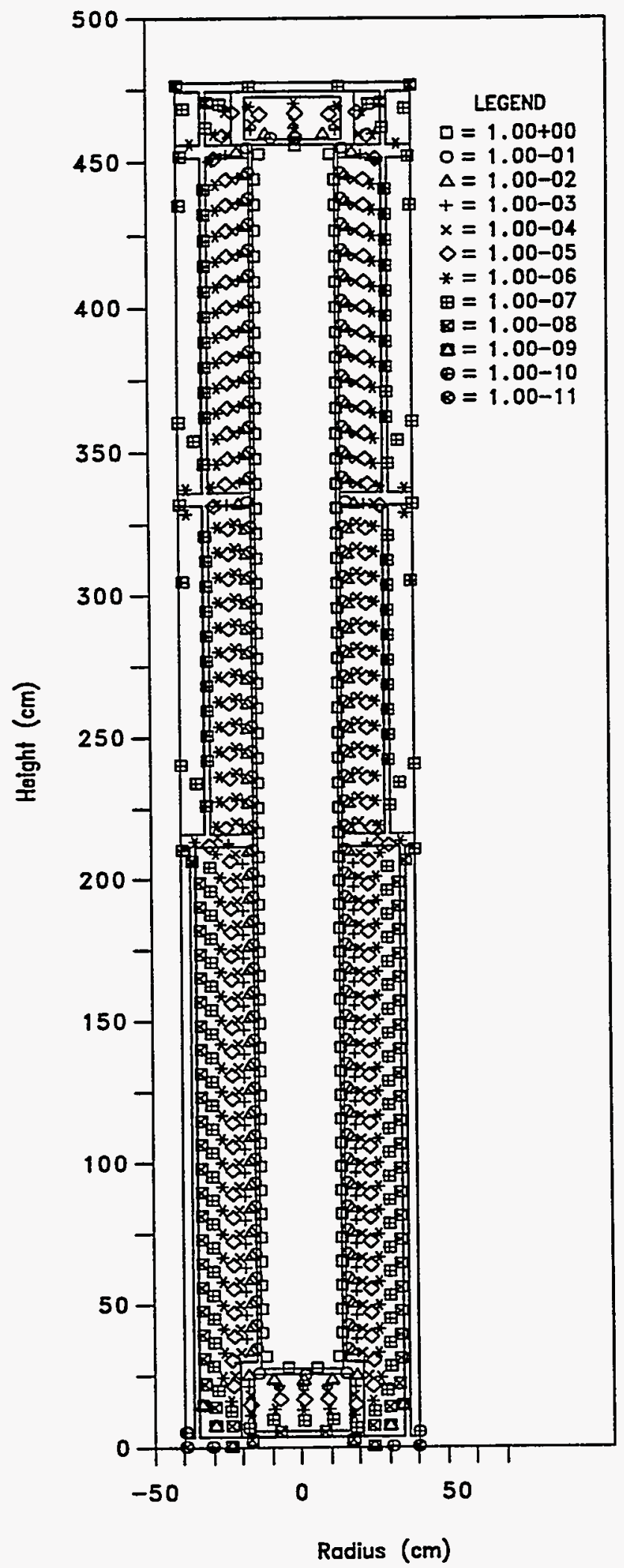

Figure D.46 Isodose Contours for the ORR MFP (20\% Enriched ${ }^{235} \mathrm{U}$ ) Source Packaged in the 10-Inch ORR Experiment Removal Shield SNF Shipping Cask. [Dose units are $\mathrm{rem} /(\mathrm{h} \cdot \mathrm{Ci})$.]

D-48 


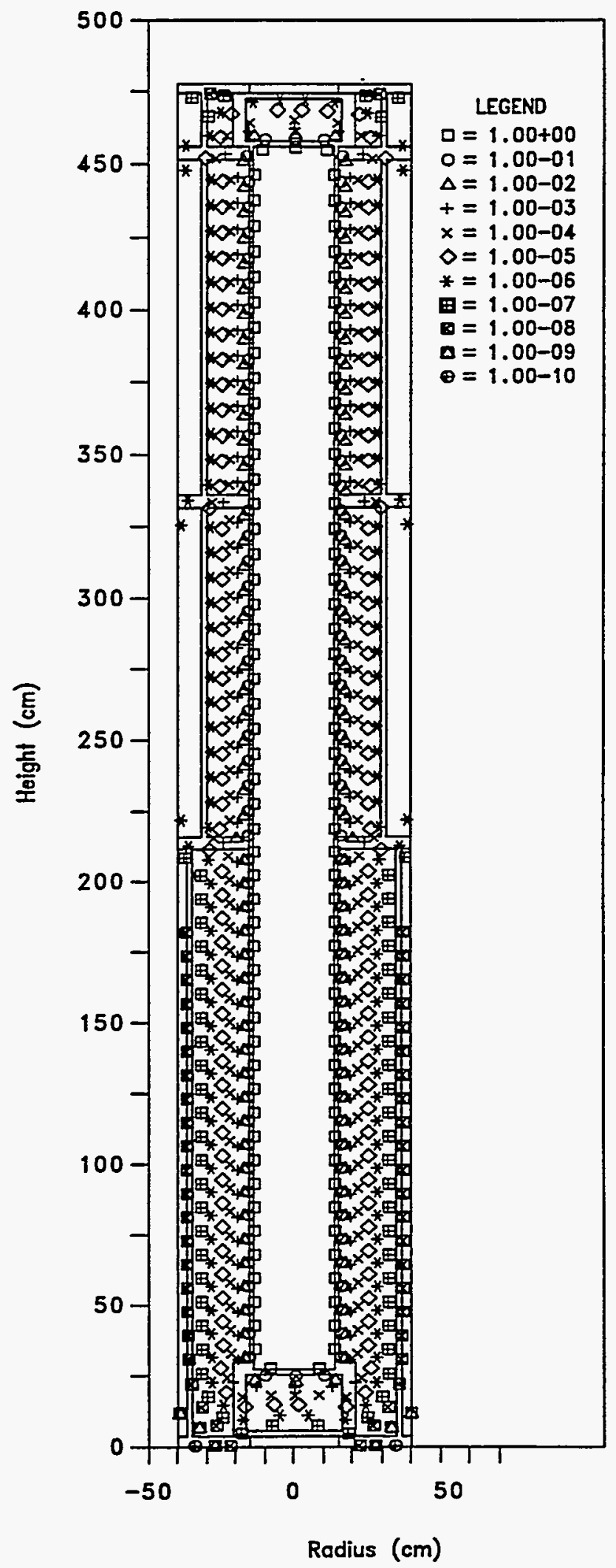

Figure D.47 Isodose Contours for the ORR MFP (40\% Enriched ${ }^{235} \mathrm{U}$ ) Source Packaged in the 10-Inch ORR Experiment Removal Shield SNF Shipping Cask. [Dose units are rem/(h-Ci).]

D-49 


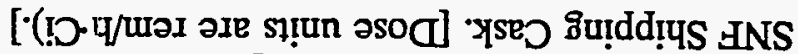

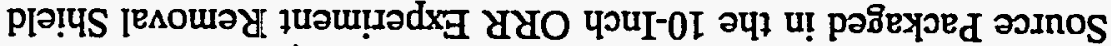

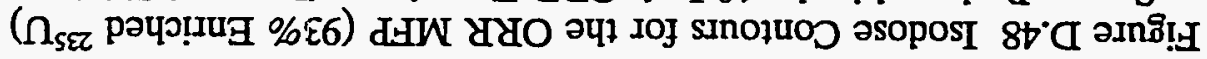

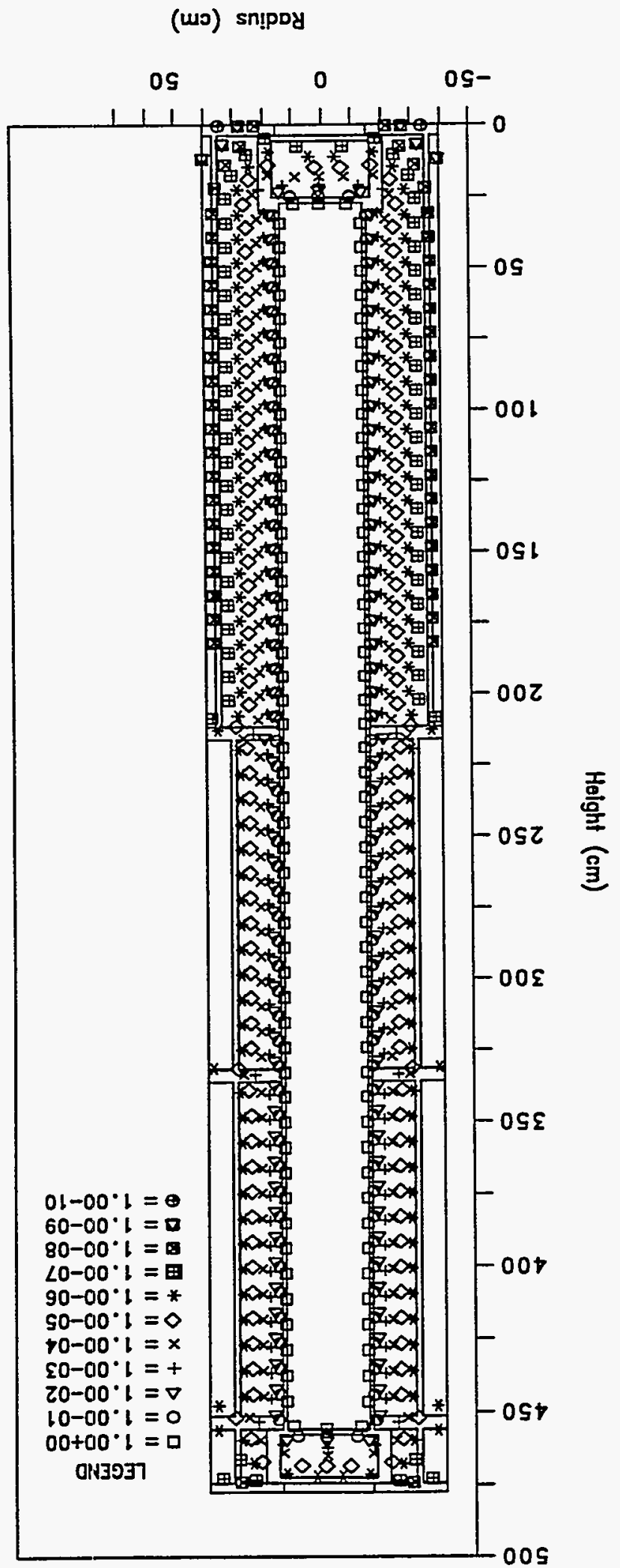




\section{APPENDIX E}

Sample Card Input Data Sets for the Computer Codes Used in the Shielding Analysis of the SNF Shipping Casks 

$\# ! / \mathrm{bin} / \mathrm{ksh}$

set $-\mathrm{XV}$

date

\#**** PALSFN1.KSH, 04/07/95, 1543 hrs ****

$\mathrm{jvp}=/ \mathrm{u} 12 / \mathrm{jvp} / \mathrm{snf}$

tmp=/u7/jvp

mkdir \$tmp/tdir\$\$

cd \$tmp/tdir\$\$

\# Korn shell script to execute a SCALE job

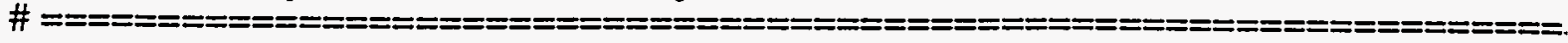

\# if you want to create a modified version of a module, DO IT HERE, not later!

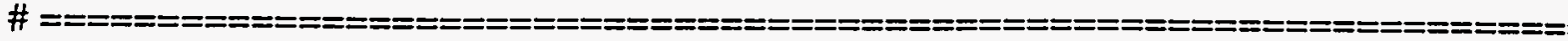

scale.mod \#/usr/epmnas/jvp/bin/scale.mod) links SCALE modules

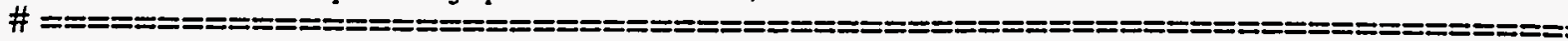

\# if you want to create a modified xsect dataset. DO IT HERE, not later!

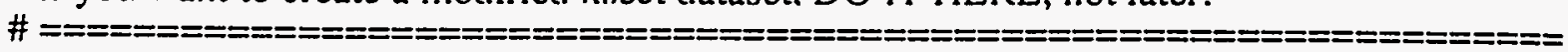

$\ln -\mathrm{s} / \mathrm{u} 31 / \mathrm{jvp} / \mathrm{xs} / \mathrm{velm} 61$.ampx.bin ft4lf()01

\# $\mathrm{ft} 47 \mathrm{f}()(0) \mathrm{i}$ is necessary for many of the ampx modules

In -s/usr/epmnas/jvp/scale/ampx.grp.bnds.bin.ft47f(0)1 ft47f001

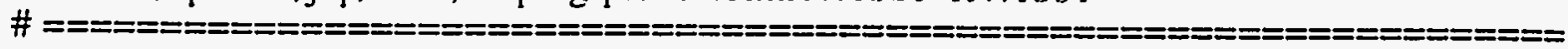

\# following touch commands maybe necessary:

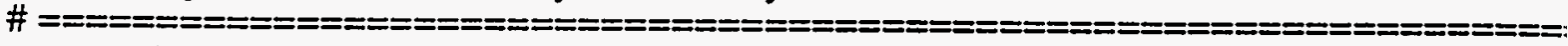

cat $>$ sysin $\ll$ 'EOF'

$=$ pal

'PAL - Read \& punch I-d Kerma factors for C,Al,Si,Cr,Mn,Fe,Ni,Pb

Input master $=41$

(1)\$ $\$$--> mmt $=$ input master, $m w t=$ input working, $n 7=$ punch device

$18 \$ 4107$

1 $\$ \$ \$,->$ npunch=\# punched nuclides, iform=( $/ 1=$ fixed/free-form,icore=words

$1 \$ \$ \& 15+4$

$28 \$$ - $->$ mt numbers to be punched

$2 \$ \$ 1527 \mathrm{fO}$

$\mathrm{t}$

' $3 \$ \$ \$$--> nuclide id's

$3 \$ \$ 13(06011313011314011324011325() 2132603132802138202 \mathrm{t}$

end

EOF

rm print _prt* $1 \$ p r t *$ out* I\$out*

touch Siout

touch \\$prt

time scule

cat print_prt* 1 pprt*_out* $\backslash$ \$out* $>$ \$jvp/palsnf1.out

mv l\$pun \$jvp/palsnfl.pun

cd Sjvp

rm -rf $\$$ tmp/tdir $\$ \$$

date

Figure E.1 Job Input Stream for the PAL Cross-Section and Activity Manipulation Program. 


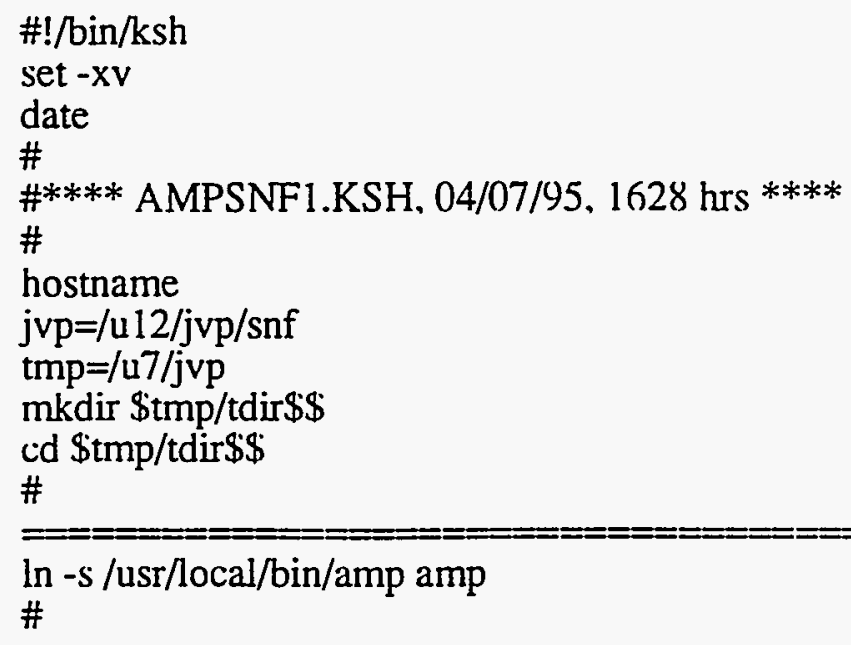

Figure E.2 Job Input Stream for the AMP Cross-Section and Activity Manipulation Program. 
' nuclide 132401 process 1527

$61 z$

243039-4 171337-4 143357-4 13246-3 117037-4 974615-5 790011-5 618606-5

497809-5 421564-5 346332-5 267694-5 19667-4 156368-5 135993-5 114174-5

920502-6 766434-6 10916-4 205257-5 447502-5 154268-4 631279-4

, nuclide 132502 process 1527

$61 \mathrm{z}$

259527-4 182273-4 152145-4 140443-4 123894-4 102911-4 831582-5 648857-5

520653-5 440177-5 36123-4 279168-5 205329-5 163529-5 142469-5 12005-4

976606-6 843676-6 127342-5 243213-5 530874-5 181611-4 733396-4

, nuclide 132603 process 1527

$61 z$

27644-3 193491-4 161191-4 148642-4 130912-4 108467-4 873881-5 679528-5

543705-5 458907-5 376197-5 290704-5 214076-5 170813-5 149099-5 126136-5

103602-5 929146-6 1479-3 286135-5 624789-5 212007-4 845638-4

, nuclide 132802 process 1527

$61 \mathrm{z}$

311782-4 216776-4 179888-4 165581-4 145398-4 119915-4 960677-5 742041-5 590438-5 496757-5 406364-5 313986-5 23188-4 18581-4 162895-5 139041-5

$116634-5$ 11284-4 197169-5 388885-5 848058-5 283217-4 110153-3

, nuclide 138202 process 1527

$61 \mathrm{z}$

188925-3 123943-3 994102-4 900713-4 769587-4 606564-4 45817-3 32839-3

244787-4 199444-4 163691-4 142376-4 141454-4 153794-4 169002-4 200559-4 270027-4 540909-4 130142-3 114175-3 102499-3 291968-3 573929-3

$\mathrm{t}$

stop

EOF

time ./amp $<$ ampinp $>$ ampout

Is - LI

mv ampout \$jvp/ampsnf1.out

mv fort.7 \$jvp/ampsnf1.pun

cd \$jvp

$\mathrm{rm}-\mathrm{rf} \$ \mathrm{tmp} / \mathrm{tdir} \$ \$$

date

Figure E.2 Continued.

E-5 


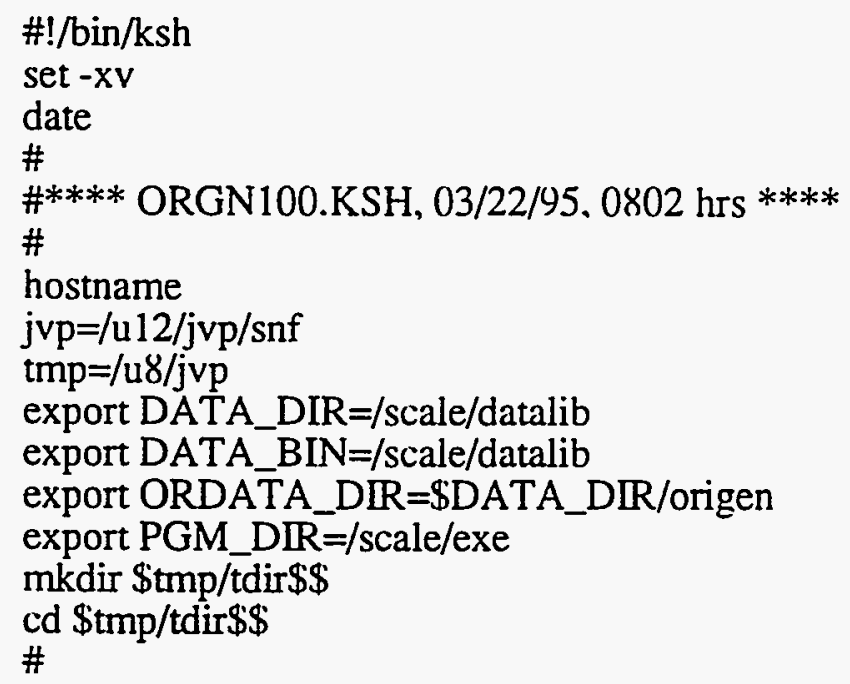

Figure E.3 Job Input Stream for the ORIGEN-S Depletion/Burnup Calculation to Define the LWR MFP (25 MWd/kgU) Source Used in the SNF Shipping Casks Shielding Analysis. 


\section{=origns}

. 25 GWD/MT, 18MW/MT. U-wt\%(u234=.021. u235=2.4()). u236=.011,

, u238=97.568): continuous opn $=1388.89$ d: Tbl A.4. ORNL-6698.

- Clad is Zircaloy: Wt\%(97.91 Zr, 1.59 Sn, 0.5 Fe -> SCALE 4.2 $1 \$ \$ 1$

Composition Library). See Tb1 3.11, ORNL-6698 for composition.

$1 \mathrm{t}$

25GWD/MT,18MW/MT,U-wt\%(234=.0921,235=2.400.236=.011,238=97.568);1338.89 d

$3 \$ \$ 31$ a3 2 a33 $23 \mathrm{e}$

$2 \mathrm{t}$

$35 \$ \$ 0$

$4 \mathrm{t}$

$56 \$ \$ 10$ a13743021 e

$57 * *$ a3 $1-10$ e

$5 \mathrm{t}$

IRRADIATION (25GWD/MT, 18MW/MT,2.4\% U235, continuous opn=1388.89 d)

MT of HM (g-atoms)

$58 * * 10 \mathrm{r} 18$

, $1 / 4$ y for $1 y$ (4 steps), 1/3 y for 1 y(3 steps),

, $1 / 2$ y for ly (2 steps), .8y for .8y (1 step).

$60 * * 2 \mathrm{i} 91.912 \mathrm{i} 365.251 \mathrm{i} 730.501095 .751388 .89$

$66 \$ \$ 1$ a5 1 a9 1 e

$73 \$ \$ 260000400000500000922340922350922360922380$

$74 * * 20.222422 .7130 .240 .90102 .130 .474099 .50$

$75 \$ \$ 3 \mathrm{r} 4 \mathrm{f} 2$

$6 \mathrm{t}$

$56 \$ \$ 010$ a10 10 al4 53 al7 2 e

$5 t$

DECAY (25GWD/MT,18MW/MT,2.4\% U235,continuous opn=1388.89 d)

MT of HM (g-atoms)

$60 * * 17$ i5 45

$61 * * 1-10$ a3 3 rl-10 e

$65 \$ \$ 1$ a7 1 a11 1 a14 1 a22 1 a28 1 a32 1 a35 1 7z lq 21

$81 \$ \$ 20261$ e $82 \$ \$$ f2

$83 * *$

$14+61+78+675+57+66+65+64+63+625+52+615+51+67+56+551+4$

$4+53+515+41+57+445+32+41+4$

$6 \mathrm{t}$

IY Decay G-Spec(VELM23 Grp;25GWD/MT,18MW/MT;Cont opn=1388.89d;Tbl A4,ORNL-6698)

5Y Decay G-Spec(VELM23 Grp;25GWD/MT,18MW/MT;Cont opn=1388.89d;Tbl A4.ORNL-6698)

$10 Y$ Decay G-Spec(VELM23 Grp;25GWD/MT,18MW/MT:Cont opn=1388.89d;Tbl A4,ORNL-6698)

15Y Decay G-Spec(VELM23 Grp;25GWD/MT,18MW/MT;Cont opn=1388.89d;Tbl A4,ORNL-6698)

20Y Decay G-Spec(VELM23 Grp;25GWD/MT,18MW/MT;Cont opn=1388.89d;Tbl A4,ORNL-6698)

25Y Decay G-Spec(VELM23 Grp;25GWD/MT,18MW/MT;Cont opn=1388.89d;Tb1 A4,ORNL-6698)

30Y Decay G-Spec(VELM23 Grp;25GWD/MT,18MW/MT;Cont opn=1388.89d;Tbl A4,ORNL-6698)

35Y Decay G-Spec(VELM23 Grp;25GWD/MT,18MW/MT;Cont opn=1388.89d;Tbl A4,ORNL-6698)

40Y Decay G-Spec(VELM23 Grp;25GWD/MT,18MW/MT;Cont opn=1388.89d;Tbl A4,ORNL-6698) 45Y Deciay G-Spec(VELM23 Grp;25GWD/MT,18MW/MT;Cont opn=1388.89d;Tb1 A4,ORNL-6698) $56 \$ \$ \mathrm{fl} \quad 5 \mathrm{t}$

end

Figure E.3 Continued. 
EOF

time ./scale

Is -1

cat \$DATA_DIR/scale.messages > orgnout

cat $\$$ tmp/tdir $\$ \$ /$ print $>>$ orgnout

cat $\$$ tmp/tdir\$\$/_prt* >> orgnout

cat $\$$ tmp/tdir\$\$/_out* $>>$ orgnout

mv orgnout \$jvp/orgn100.out

cd \$jvp

$\mathrm{rm}-\mathrm{rf} \$ \mathrm{tmp} / \mathrm{tdir} \$ \$$

date

Figure E.3 Continued.

E-8 


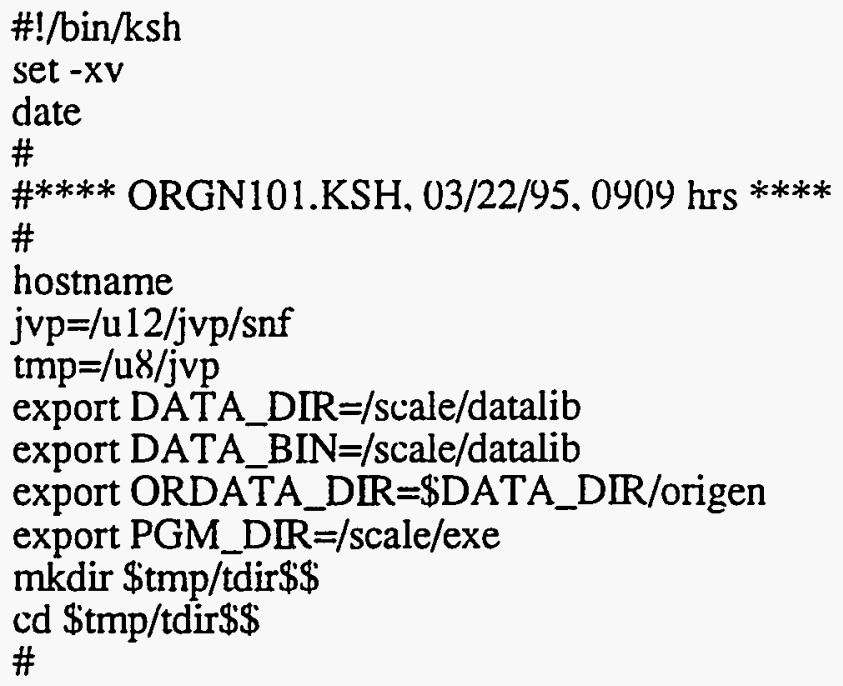

Figure E.4 Job Input Stream for the ORIGEN-S Depletion/Burnup Calculation to Define the LWR MFP (50 MWd/kgU) Source Used in the SNF Shipping Casks Shielding Analysis. 
$=$ origns

' 50 GWD/MTU, 40MW/MTU, U-wt\%(u234=.037, u235=4.200, u236=.019.

- u238=95.744); continuous opn $=1250.00 \mathrm{~d}$ : Tbl A.4. ORNL-6698.

- Cycle 3 of 5 .

, Clad is Zircaloy: Wt\%(97.91 Zr, $1.59 \mathrm{Sn}, 0.5 \mathrm{Fe}$-> SCALE 4.2 $1 \$ \$ 1$

Composition Library). See Tbl 3.11. ORNL-6698 for composition.

$1 \mathrm{t}$

25GWD/MTU,18MW/MTU,U-wt\%(234=.0371.235=4.200.236=.019.238=95.744); $1250.00 \mathrm{~d}$

$3 \$ \$ 31$ a3 3 a33 $23 \mathrm{e}$

$2 \mathrm{t}$

$35 \$ \$ 0$

$4 \mathrm{t}$

$56 \$ \$ 10$ a13743021e

$57 * *$ a3 $1-10 \mathrm{e}$

$5 t$

IRRADIATION (50GWD/MTU,40MW/MTU,4.2\% U235,continuous opn=1250.00 d)

MT of HM (g-atoms)

$58 * * 10 \mathrm{r} 40$

, $1 / 4$ y for $1 y$ (4 steps), 1/3 y for 1 y(3 steps),

, $1 / 2$ y for ly (2 steps), $.42 y$ for $.42 y$ (1 step).

$60 * * 2 \mathrm{i} 91.912 \mathrm{i} 365.251 \mathrm{i} 730.501095 .751250 .00$

$66 \$ \$ 1$ a5 1 a9 1 e

$73 \$ \$ 260000400000500000922340922350922360922380$

$74 * * 20.222422 .7130 .241 .58178 .720 .814022 .86$

$75 \$ \$ 3 \mathrm{r} 4 \mathrm{f} 2$

$6 \mathrm{t}$

$56 \$ \$ 010$ a10 10 a14 53 a172 e

$5 \mathrm{t}$

DECAY (50GWD/MTU,40MW/MTU,4.2\% U235, continuous opn=1250.00 d)

MT of HM (g-atoms)

$60 * * 17 \mathrm{i} 545$

$61 * * 1-10$ a3 $3 r 1-10 \mathrm{e}$

$65 \$ \$ 1$ a7 1 a11 1 al4 1 a22 1 a28 1 a32 1 a35 1 7z 1 q21

$81 \$ \$ 20261$ e $82 \$ \$ f 2$

$83 * *$

$14+61+78+675+57+66+65+64+63+625+52+615+51+67+56+551+4$

$4+53+515+41+57+445+32+41+4$

$6 \mathrm{t}$

IY Decay G-Spec(VELM23 Grp;50GWD/MT,40MW/MT;Cont opn=1250.00d;Tbl A4,ORNL-6698)

5Y Decay G-Spec(VELM23 Grp;50GWD/MT,40MW/MT;Cont opn=1250.00d;Tbl A4,ORNL-6698)

10Y Decay G-Spec(VELM23 Grp;50GWD/MT,40MW/MT;Cont opn=1250.00d;Tbl A4,ORNL-6698)

15Y Decay G-Spec(VELM23 Grp;50GWD/MT,40MW/MT;Cont opn=1250.00d;Tbl A4,ORNL-6698)

20Y Decay G-Spec(VELM23 Grp;50GWD/MT,40MW/MT;Cont opn=1250.00d;Tbl A4,ORNL-6698)

25Y Decay G-Spec(VELM23 Grp;50GWD/MT,40MW/MT;Cont opn=1250.00d:Tbl A4,ORNL-6698)

30Y Decay G-Spec(VELM23 Grp;50GWD/MT,40MW/MT;Cont opn=1250.00d;Tbl A4,ORNL-6698)

35Y Decay G-Spec(VELM23 Grp;50GWD/MT,40MW/MT;Cont opn=1250.00d;Tbl A4,ORNL-6698)

40Y Decay G-Spec(VELM23 Grp;50GWD/MT,40MW/MT;Cont opn=1250.00d;Tbl A4,ORNL-6698);

45Y Decay G-Spec(VELM23 Grp;50GWD/MT.40MW/MT:Cont opn=1250.(00d;Tbl A4,ORNL-6698;

Figure E.4 Continued.

E-10 
$56 \$ \$$ fo $5 t$

end

EOF

time ./scale

Is -1

cat \$DATA_DIR/scale.messages > orgnout

cat $\$$ tmp/tdir $\$ \$ /$ print $\gg$ orgnout

cat $\$$ tmp/tdir $\$ \$ /$ prt* $>$ orgnout

cat $\$$ tmp/tdir\$\$/_out* $>$ orgnout

mv orgnout $\$$ jvp/orgn 101.out

cd \$jvp

rm -rf \$tmp/tdir\$\$

date

Figure E.4 Continued.

E-11 
$\# ! / \mathrm{bin} / \mathrm{ksh}$

set -xv

date

$\#$

\#**** ORGN13A.KSH, 04/26/95, 1205 hrs ****

\#

hostname

jvp $=/ \mathrm{u} 12 / \mathrm{jvp} / \mathrm{snf}$

tmp $=/ \mathrm{u} 8 / \mathrm{jvp}$

export DATA_DIR=/scale/datalib

export DATA_BIN=/scale/datalib

export ORDATA_DIR=\$DATA_DIR/origen

export PGM_DIR=/scale/exe

mkdir \$tmp/tdir\$\$

cd $\$$ tmp/tdir $\$ \$$

\#

In -s \$PGM_DIR/scale scale

ln $-s$ \$PGM_DIR/csas csas

In -s \$PGM_DIR/origns origns

ln $-s$ \$ORDATA_DIR/binrylib/prlimlwr ft21f001

In -s \$ORDATA_DIR/binrylib/pwr33gwd ft22f001

In -s \$ORDATA_DIR/binrylib/maphnobr ft23f001

In -s \$ORDATA_DIR/binrylib/maphh2ob ft24f00 1

In -s \$ORDATA_DIR/binrylib/basimfbr ft25f001

in -s \$ORDATA_DIR/binrylib/maphuo2b ft26f001

In -s \$ORDATA_DIR/cardlib/end6dec ft27f001

In -s \$ORDATA_DIR/cardlib/xsectpho ft28f001

In -s \$ORDATA_DIR/binrylib/P2518.lib ft31f001

\#In -s \$DATA_DIR/scale.rev02.xn238 ft70f001

In -s \$DATA_KENO/albedos ft79f001

In -s \$DATA_KENO/weights ft80f001

In -s \$DATA_BIN/scale.rev02.xn16 ft81f00

in $-\$$ \$DATA_BIN/scale.rev03.xn27 ft82f001

ln -s \$DATA_BIN/scale.rev02.xn123 ft83f001

in -s \$DATA_BIN/scale.rev03.xn218 ft84f001

In $-\mathrm{s}$ \$DATA_BIN/scale.rev02.xn22g $18 \mathrm{ft} 85 \mathrm{f001}$

In -s \$DATA_BIN/scale.rev02.xg18 ft86f001

in -s \$DATA_BIN/scale.rev03.xn27burn ft87f001

In -s \$DATA_BIN/scale.rev03.xn27g18 ft88f001

In -s \$DATA_BIN/scale.rev05.sclib ft89f001

In -s \$DATA_DIR/h7matlib h7matlib

In -s \$DATA_DIR/qatable qatable

In $-s$ \$DATA_DIR/aliases aliases

Is $-\mathrm{Ll}$

\#

cat $>$ sysin $<$ 'EOF' $^{\prime}$

Figure E.5 Job Input Stream for the ORIGEN-S Depletion/Burnup Calculation to Define the ORR MFP (20\% Enriched ${ }^{235} U$ ) Source

Used in the SNF Shipping Casks Shielding Analysis. 
=origns

' ORR High-U-Loaded Fuel Element DevU(HFED); ORR operated at $30 \mathrm{MW}$ FP,

, $93 \%$ enrichment, 220 gU235/element, 30 elemts, per R. Hobbs, 3/22/95.

, Cycle 2 of 3, P2518 Lib.

, Used tables 1.1, 3.2. B.1, B.5. B.16. B.20, and B.24 in "Summary

, Report of the HFED Mini-plate Irradiations for the RERTR Pgm," $1 \$ \$ 1$ ORNL-6539, Apr 89.

$1 \mathrm{t}$

U6Fe, Module 23, Slot 6, U235=.1984g, U238=.8016g, Fe=.042g; 91d; P2518 lib

$3 \$ \$ 31$ a3 2 a33 $23 \mathrm{e}$

$2 \mathrm{t}$

$35 \$ \$ 0$

$4 \mathrm{t}$

$56 \$ 10$ a13 $343021 \mathrm{e}$

$57 * * 0$ a3 $1-10 \mathrm{e}$

5 t

IRRADIATION: $9.1-91 \mathrm{~d}$ of $91 \mathrm{~d}$ total

$\mathrm{G}$ of HM (g-atoms)

$58 * * 10 \mathrm{r} 5.59-4$

$60 * * 8 \mathrm{i} 9.191$

$66 \$ \$ 1$ a5 1 e

$73 \$ \$ 26+4922350922380$

$74 * * 7.52-48.44-4$ 3.37-3

$75 \$ \$ 42$

$6 \mathrm{t}$

$56 \$ \$ 010$ a10 10 a1453 a17 e

$5 \mathrm{t}$

DECAY (91 d Irrad)

$\mathrm{G}$ of HM (g-atoms)

$60 * * 17$ i5 45

$61 * * 1-10$ a3 $3 \mathrm{r} 1-10 \mathrm{e}$

$65 \$ \$ 1$ a7 1 al1 1 al4 1 a22 1 a28 1 a32 1 a35 1 7z lq21

$81 \$ \$ 20261$ e $\$ 2 \$ \$ \mathrm{f} 2$

$83^{* *}$

$14+61+78+675+57+66+65+64+63+625+52+615+51+67+56+551+4$

$4+53+515+41+57+445+32+41+4$

$6 \mathrm{t}$

$1 Y$ Decay G-Spec(VELM23 Gp;1.99MWD/gU,5.59-4MW/gU;Cont opn=91d;ORNL-6539)

5Y Decay G-Spec(VELM23 Gp;1.99MWD/gU,5.59-4MW/gU;Cont opn=91d;ORNL-6539)

10Y Decay G-Spec(VELM23 Gp;1.99MWD/gU,5.59-4MW/gU;Cont opn=91d;ORNL-6539)

$15 Y$ Decay G-Spec(VELM23 Gp;1.99MWD/gU,5.59-4MW/gU;Cont opn=91d;ORNL-6539)

20Y Decay G-Spec(VELM23 Gp;1.99MWD/gU,5.59-4MW/gU;Cont opn=91d;ORNL-6539)

25Y Decay G-Spec(VELM23 Gp;1.99MWD/gU,5.59-4MW/gU;Cont opn=91d;ORNL-6539)

30Y Decay G-Spec(VELM23 Gp;1.99MWD/gU,5.59-4MW/gU;Cont opn=91d;ORNL-6539)

35Y Decay G-Spec(VELM23 Gp;1.99MWD/gU,5.59-4MW/gU;Cont opn=91d;ORNL-6539)

40Y Decay G-Spec(VELM23 Gp;1.99MWD/gU,5.59-4MW/gU;Cont opn=91d;ORNL-6539)

45Y Decay G-Spec(VELM23 Gp;1.99MWD/gU,5.59-4MW/gU;Cont opn=91d;ORNL-6539)

Figure E.5 Continued. 
$56 \$ \$$ fo $5 t$

end

EOF

time.$/$ scale

Is -1

cat \$DATA_DIR/scale.messages > orgnout

cat $\$$ tmp/tdir $\$ \$ /$ print $\gg$ orgnout

cat $\$$ tmp/tdir $\$ \$ /$ prt* $>$ orgnout

cat $\$$ tmp/tdir $\$ \$$ /_out* $>$ > orgnout

mv orgnout $\$ j v p / o r g n 13 a . o u t$

cd \$jvp

rm -rf \$tmp/tdir\$\$

date

Figure E.5 Continued.

E-14 


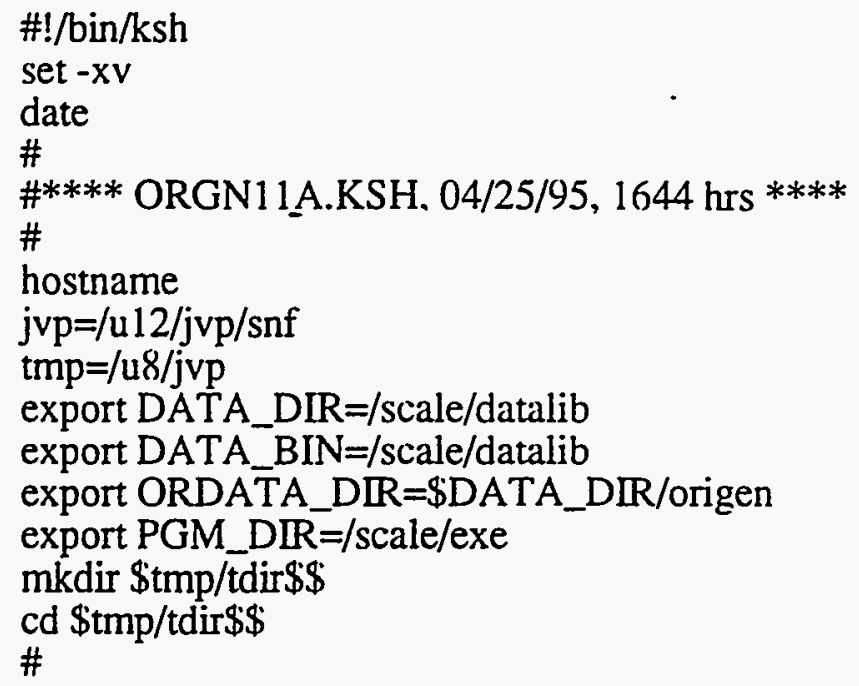

Figure E.6 Job Input Stream for the ORIGEN-S Depletion/Burnup Calculation to Define the ORR MFP (40\% Enriched ${ }^{235} U$ ) Source Used in the SNF Shipping Casks Shielding Analysis. 
$=$ origns

' ORR High-U-Loaded Fuel Element Dev()(HFED); ORR operated at $30 \mathrm{MW}$ FP.

, 93\% enrichment, 220 gU235/element, 30 elemts, per R. Hobbs, 3/22/95.

, $\quad$ Cycle 2 of 3, P2518 Lib.

- Used tables 1.1, 3.2. B.1. B.5, B.16. B.20, and B.24 in "Summary

' Report of the HFED Mini=plate Irradiations for the RERTR Pgm,"

$1 \$ \$ 1$

ORNL-6539, Apr 89.

$1 \mathrm{t}$

UAl, Module 1, Slot 1, U235=2.201g, U238=3.269g, Al=.37g; 470d; P2518 lib

$3 \$ \$ 31$ a3 2 a33 $23 \mathrm{e}$

$2 \mathrm{t}$

$35 \$ \$ 0$

$4 \mathrm{t}$

$56 \$ \$ 10$ al3 $343021 \mathrm{e}$

$57 * * 0$ a3 $1-10 \mathrm{e}$

$5 \mathrm{t}$

IRRADIATION: $10-100 \mathrm{~d}$ of $470 \mathrm{~d}$ total

$\mathrm{G}$ of HM (g-atoms)

$58 * * 10 \mathrm{r} 7.65-4$

$60 * * 8 \mathrm{i} 10100$

$66 \$ \$ 1$ a5 1 e

$73 \$ \$ 130270922350922380$

$74 * * 1.37-2$ 1.71-3 2.51-3

$75 \$ \$ 4 \mathrm{f} 2$

$6 \mathrm{t}$

$56 \$ \$ 10$ a10 10 a14 43021 e

$57 * * 100$ a3 $1-10 \mathrm{e}$

$5 \mathrm{t}$

IRRADIATION: $110-200 \mathrm{~d}$ of $470 \mathrm{~d}$ total

$\mathrm{G}$ of HM (g-atoms)

$58 * * 10$ r $7.65-4$

$60 * * 8 \mathrm{il} 10200$

$66 \$ \$ 1$ a5 1 e

$6 \mathrm{t}$

$56 \$ \$ 10$ a10 10 a14 $43021 \mathrm{e}$

$57 * * 200$ a3 $1-10$ e

$5 t$

IRRADIATION: $210-300 \mathrm{~d}$ of $470 \mathrm{~d}$ total

$\mathrm{G}$ of $\mathrm{HM}$ (g-atoms)

$58 * * 10 \mathrm{r} 7.65-4$

$60 * * 8 \mathrm{i} 210300$

$66 \$ \$ 1$ a5 1 e

$6 \mathrm{t}$

$56 \$ \$ 10$ a10 10 a1443021e

$57 * * 300$ a3 $1-10 \mathrm{e}$

$5 \mathrm{t}$

IRRADIATION: $310-400 \mathrm{~d}$ of $470 \mathrm{~d}$ total

$\mathrm{G}$ of $\mathrm{HM}$ (g-atoms)

Figure E.6 Continued. 
$58 * * 10 \mathrm{r} 7.65-4$

$60 * * 8 \mathrm{i} 310400$

$66 \$ \$ 1$ a5 1 e

$6 \mathrm{t}$

$56 \$ \$ 2$ r7 a10 10 a14 $43021 \mathrm{e}$

$57 * * 400$ a3 $1-10$ e

$5 \mathrm{t}$

IRRADIATION: $410-470 \mathrm{~d}$ of $470 \mathrm{~d}$ total

$\mathrm{G}$ of $\mathrm{HM}$ (g-atoms)

$58 * * 7 \mathrm{r} 7.65-4$

$60 * * 5 i 410470$

$66 \$ \$ 1$ a5 1 e

$6 \mathrm{t}$

$56 \$ \$ 010$ a10 al4 53 a17 2 e

$5 t$

DECAY (470 d Irrad)

$\mathrm{G}$ of HM (g-atoms)

$60 * * 17 \mathrm{i} 545$

$61 * * 1-10$ a3 3r $1-10$ e

$65 \$ \$ 1$ a7 1 a11 1 a14 1 a22 1 a28 1 a32 1 a35 1 7z lq21

$81 \$ \$ 20261$ e $82 \$ \$$ f2

$83^{* *}$

$14+61+78+675+57+66+65+64+63+625+52+615+51+67+56+551+4$

$4+53+515+41+57+445+32+41+4$

$6 \mathrm{t}$

1Y Decay G-Spec(VELM23 Gp;1.99MWD/gU,7.65-4MW/gU;Cont opn=470d;ORNL-6539)

5Y Decay G-Spec(VELM23 Gp;1.99MWD/gU,7.65-4MW/gU;Cont opn=470d;ORNL-6539)

10Y Decay G-Spec(VELM23 Gp;1.99MWD/gU,7.65-4MW/gU;Cont opn=470d;ORNL-6539)

15Y Decay G-Spec(VELM23 Gp;1.99MWD/gU,7.65-4MW/gU;Cont opn=470d;ORNL-6539)

20Y Decay G-Spec(VELM23 Gp;1.99MWD/gU.7.65-4MW/gU;Cont opn=470d;ORNL-6539)

25Y Decay G-Spec(VELM23 Gp;1.99MWD/gU,7.65-4MW/gU;Cont opn=470d;ORNL-6539)

30Y Decay G-Spec(VELM23 Gp;1.99MWD/gU.7.65-4MW/gU;Cont opn=470d;ORNL-6539)

35Y Decay G-Spec(VELM23 Gp;1.99MWD/gU,7.65-4MW/gU;Cont opn=470d;ORNL-6539)

40Y Decay G-Spec(VELM23 Gp;1.99MWD/gU,7.65-4MW/gU;Cont opn=470d;ORNL-6539)

45Y Decay G-Spec(VELM23 Gp;1.99MWD/gU,7.65-4MW/gU;Cont opn=470d;ORNL-6539)

$56 \$ \$$ fo $5 t$

end

EOF

time ./scale

Is -1

cat \$DATA_DIR/scale.messages $>$ orgnout

cat $\$$ tmp/tdir $\$ \$ /$ print $\gg$ orgnout

cat $\$$ tmp/tdir $\$ \$ /$ prt* $>>$ orgnout

cat \$tmp/tdir\$\$/_out* > orgnout

mv orgnout $\$ j v p / o r g n 1$ la.out

cd \$jvp

rm -rf \$tmp/tdir\$\$

date

Figure E.6 Continued. 


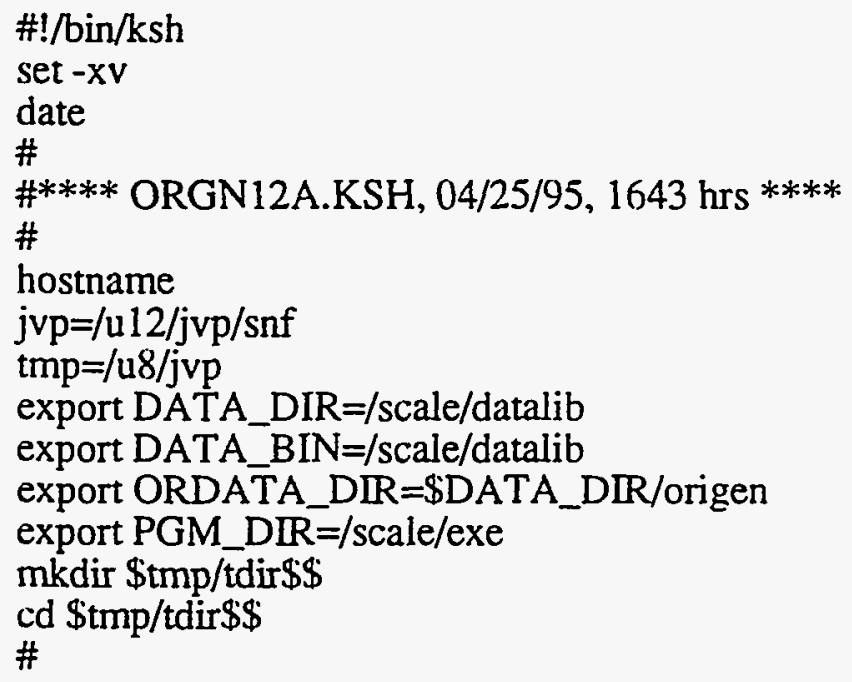

Figure E. $7^{-}$Job Input Stream for the ORIGEN-S Depletion/Burnup

Calculation to Define the ORR MFP (93\% Enriched ${ }^{235} \mathrm{U}$ )

Source Used in the SNF Shipping Casks Shielding Analysis. 
' ORR High-U-Loaded Fuel Element Dev()(HFED); ORR operated at $30 \mathrm{MW}$ FP,

, 93\% enrichment. 220 gU235/element, 30 elemts, per R. Hobbs, 3/22/95.

, Cycle 2 of 3. P2518 Lib.

- Used tables 1.1, 3.2, B.1, B.5, B.16, B.20. and B.24 in "Summary

, Report of the HFED Mini-plate Irradiations for the RERTR Pgm,"

$1 \$ \$ 1$

ORNL-6539, Apr 89.

$1 \mathrm{t}$

U3O8, Module 5, Slot 10, U235=1.448g, U238=0.105g, O=.181g; 268d; P2518 lib $3 \$ \$ 31$ a3 2 a33 $23 \mathrm{e}$

$$
2 \mathrm{t}
$$

$35 \$ \$ 0$

$4 \mathrm{t}$

$56 \$ \$ 10$ a13 $343021 \mathrm{e}$

$57 * * 0$ a3 $1-10 \mathrm{e}$

$5 t$

IRRADLATION: $10-100 \mathrm{~d}$ of $268 \mathrm{~d}$ total

$\mathrm{G}$ of $\mathrm{HM}$ (g-atoms)

$58 * * 10 \mathrm{r} 2.65-3$

$60 * * 8 \mathrm{i} 10100$

$66 \$ \$ 1$ a5 1 e

$73 \$ \$ 80160922350922380$

$74 * * 1.13-23.97-32.85-4$

$75 \$ \$ 4 \mathrm{f} 2$

$6 \mathrm{t}$

$56 \$ \$ 10$ a10 10 a1443021e

$57 * * 100$ a3 $1-10 \mathrm{e}$

$5 t$

IRRADIATION: $110-200 \mathrm{~d}$ of $268 \mathrm{~d}$ total

$\mathrm{G}$ of $\mathrm{HM}$ (g-atoms)

$58 * * 10 \mathrm{r} 2.65-3$

$60 * * 8 \mathrm{i} 110200$

$66 \$ \$ 1$ a5 1 e

$6 \mathrm{t}$

$56 \$ \$ 2$ r a 1010 a1443021e

$57 * * 200$ a3 $1-10$ e

$5 \mathrm{t}$

IRRADIATION: $210-268 \mathrm{~d}$ of $268 \mathrm{~d}$ total

$\mathrm{G}$ of $\mathrm{HM}$ (g-atoms)

$58 * * 7 \mathrm{r} 2.65-3$

$60 * * 4 \mathrm{i} 210260268$

$66 \$ \$ 1$ a5 1 e

$6 t$

$56 \$ \$ 010$ a10 7 a1453 a17 2 e

$5 t$

DECAY (268 d Irrad)

$\mathrm{G}$ of HM (g-atoms)

Figure E.7 Continued.

E-19 
$60 * * 17 \mathrm{i} 545$

$61 * * 1-10$ a3 $3 \mathrm{r} 1-10 \mathrm{e}$

$65 \$ \$ 1$ a7 1 a11 1 a14 1 a22 1 a28 1 a32 1 a35 1 7z lq 21

$81 \$ \$ 20261$ e $82 \$ \$ f 2$

$83 * *$

$14+61+78+675+57+66+65+64+63+625+52+615+51+67+56+551+4$

$4+53+515+41+57+445+32+41+4$

$6 \mathrm{t}$

1Y Decay G-Spec(VELM23 Gp;1.99MWD/gU,2.65-3MW/gU;Cont opn=470d;ORNL-6539)

5Y Decay G-Spec(VELM23 Gp;1.99MWD/gU,2.65-3MW/gU;Cont opn=470d;ORNL-6539)

10Y Decay G-Spec(VELM23 Gp;1.99MWD/gU,2.65-3MW'gU;Cont opn=470d;ORNL-6539)

15Y Decay G-Spec(VELM23 Gp;1.99MWD/gU,2.65-3MW/gU;Cont opn=470d;ORNL-6539)

20Y Decay G-Spec(VELM23 Gp;1.99MWD/gU,2.65-3MW/gU;Cont opn=470d;ORNL-6539)

25Y Decay G-Spec(VELM23 Gp;1.99MWD/gU,2.65-3MW/gU;Cont opn=470d;ORNL-6539)

30Y Decay G-Spec(VELM23 Gp;1.99MWD/gU,2.65-3MW/gU;Cont opn=470d;ORNL-6539)

35Y Decay G-Spec(VELM23 Gp;1.99MWD/gU,2.65-3MW/gU;Cont opn=470d:ORNL-6539)

40Y Decay G-Spec(VELM23 Gp;1.99MWD/gU,2.65-3MW/gU:Cont opn=470d:ORNL-6539)

45Y Decay G-Spec(VELM23 Gp;1.99MWD/gU,2.65-3MW/gU:Cont opn=470d:ORNL-6539)

$56 \$ \$$ fo $5 \mathrm{t}$

end

EOF

time ./scale

ls -1

cat \$DATA_DIR/scale.messages > orgnout

cat $\$$ tmp/tdir $\$ \$ /$ print $\gg$ orgnout

cat $\$$ tmp/tdir\$\$/_prt* $>>$ orgnout

cat \$tmp/tdir\$\$/_out* $>$ orgnout

mv orgnout \$jvp/orgn12a.out

cd \$jvp

$\mathrm{rm}-\mathrm{rf} \$ \mathrm{tmp} / \mathrm{tdir} \$ \$$

date

Figure E.7 Continued. 
GIP mixtures for the SNF Shipping Casks calculations -- P5 forward

I\$\$ 843487162 /igm, iht,ihs,ihm,ms

$488418605 / \mathrm{mcr}, \mathrm{mtp}, \mathrm{mtm}$,ith,isct

222120 /iprt,iout,idot,nbuf

et

$10 \$ \$ 4 i 1331382 q 64 i 139144$ 5q6 $4 \mathrm{i} 1451504 \mathrm{i} 1511563 q 64 \mathrm{i} 157162$

$/$ air ss-347 lead sae-1020 aluminum

4i163 168 5q6 4i169 174 4i175 $1803 q 64$ il 181186

/ ss-347 lead sae-1020 aluminum (g-heating)

$11 \$ \$ 4 i 61664 i 67724 i 9196$ / air

$4 \mathrm{i} 55604 \mathrm{i} 79844 \mathrm{i} 971024 \mathrm{i} 1031084 \mathrm{i} 1091144 \mathrm{i} 115120$ / ss-347

4 i127 132 / lead

$4 \mathrm{i} 55604 \mathrm{i} 79844 \mathrm{i} 1031084 \mathrm{i} 109114$ / sae-1020

$4 \mathrm{i} 7378$ / aluminum

4i1 $64 \mathrm{i} 13184 \mathrm{i} 19244 \mathrm{i} 25304 \mathrm{i} 31364 \mathrm{i} 3742$ / ss-347

$4 \mathrm{i} 4348$ / lead

$4 \mathrm{i} 164 \mathrm{i} 13184 \mathrm{i} 25304 \mathrm{i} 3136$ / sae-1020

$4 i 712$ / aluminum

$12 * *$ /number densities (atoms/b-cm)

6r3.980-05 6r1.068-05 6r2.370-07 / dry air

6r3.189-04 6r1.705-03 6r1.658-02

6r1.743-03 6r5.909-02 6r8.156-03 / ss-347

6r3.296-02 / lead

6r7.883-(04 6r4.214-04 6r3.878-04 6r8.401-02 / sae-1020

6r6.027-(1)2 / aluminum

6r3.189-()4 6r1.705-03 6r1.658-02

6r1.743-03 6r5.909-02 6r8.156-03 / ss-347

6r3.296-02 / lead

6r7.883-04 6r4.214-04 6r3.878-04 6r8.401-02 / sae-1020

6r6.027-()2 / aluminum

$13 \$ \$ 1234567374757677787980818283848586878889$

90109110111112113114115116117118119120133134135

136137138139140141142143144169170171172173174175

176177178179180181182183184185186193194195196197

198229230231232233234355356357358359360

/ h,c,n,o,al,si,cl,ar,cr,mn,fe,ni,nb,pb

$\mathrm{t}$

/ c,al,si,cr,mn,fe,ni,pb g-heating factors (Watt-sec-barn/gamma-atom)

5307z 526004-18 86z 419892-18 86z 375592-18 86z 357626-18 86z 330958-18 86z 29492-17 86z 257619-18 86z 218175-18 86z 186171-18 86z 162754-18 86z 136704-18 86z 106453-18 86z 776947-19 86z 61124-18 86z 525735-19 86z 430968-19 86z 326429-19 86z 196145-19 86z 92423-19 86z 561464-20 86z 400546-20 86z 646769-20 86z 301508-19 86z

$14 * * 7308 z$

$14 * * 7308 z$

$14 * * 7308 z$

$14 * * 7308 \mathrm{z}$

$14 * * 7308 z$

Figure E.8 Card Input Data for the GIP Cross-Section and

Gamma-Ray Heating Activity Mixing Calculation. 
5307z 152161-17 86z 11363-16 86z 982247-18 86z 921357-18 86z 833591-18. 86z 719343-18 86z 607341-18 86z 497136-18 86z 413992-18 86z 3573-16 86z 297469-18 86z 230826-18 86z 168555-18 86z 13274-17 86z 114294-18 86z 939159-19 86z 716049-19 86z 449015-19 86z 279135-19 86z 305217-19 86z 531712-19 86z $188057-1886 z 896348-1886 z$ $14 * * 7308 \mathrm{z}$ $14 * * 7308 \mathrm{z}$ $14 * * 7308 \mathrm{z}$ $14 * * 7308 \mathrm{z}$ $14 * * 7308 \mathrm{z}$ $14 * *$

5307z 169693-17 86z 125831-17 86z 108353-17 86z 101463-17 86z 915573-18 86z 787063-18 86z 66165-17 86z 539159-18 86z 447506-18 86z 385533-18 86z 320577-18 86z 248642-18 86z 181603-18 86z 143066-18 86z 123231-18 86z 101342-18 86z 774399-19 86z 492358-19 86z 328922-19 86z 393386-19 86z 725776-19 86z 259633-18 86z 122037-17 86z $14 * * 7308 \mathrm{z}$ $14 * * 7308 \mathrm{z}$ $14 * * 7308 z$ $14 * * 7308 z$ $14 * * 7308 \mathrm{z}$ $14 * *$

5307z 389395-17 86z 274514-17 86z 229685-17 86z 212226-17 86z 187516-17 $86 z$ 156152-17 86z 126575-17 86z 991125-18 86z 797585-18 86z 675426-18 86z 55489-17 86z 428897-18 86z 315103-18 86z 250531-18 86z 217887-18 86z 182928-18 86z 147482-18 86z 122797-18 86z 174895-18 86z 328861-18 86z 716983-18 86z 247167-17 86z 101143-16 86z $14 * * 7308 \mathrm{z}$ $14 * * 7308 \mathrm{z}$ $14 * 7308 z$ $14 * * 7308 \mathrm{z}$ $14 * 7308 \mathrm{z}$ $14 * *$

5307z 415812-17 86z 292036-17 86z 243765-17 86z 225016-17 86z 198502-17 $86 z \quad 164883-17$ 86z $133235-17$ 86z 103959-17 86z 834185-18 86z 705247-18 $86 z$ 578759-18 86z 44728-17 86z 328976-18 86z 262005-18 86z 228262-18 86z 192343-18 86z 156471-18 86z 135173-18 86z 204026-18 86z 389673-18 86z $850561-1886 \mathrm{z} 290975-1786 \mathrm{z} 117504-1686 \mathrm{z}$ $14 * 7308 \mathrm{z}$ $14 * * 7308 \mathrm{z}$ $14 * * 7308 \mathrm{z}$ $14 * * 7308 \mathrm{z}$ $14 * 7308 \mathrm{z}$ $14 * *$

5307z 442909-17 86z 310009-17 86z 258259-17 86z 238153-17 86z 209746-17 $86 z$ 173785-17 86z 140012-17 86z 108873-17 86z 871119-18 86z 735256-18 $86 z$ 602739-18 86z 465763-18 86z 34299-17 86z 273675-18 86z 238885-18 86z 202094-18 86z 16599-17 86z 148867-18 86z 236964-18 86z 458443-18 86z $100103-1786 z 339676-1786 z 135487-1686 z$

Figure E.8 Continued. 


$$
\begin{aligned}
& 14 * * 7308 \mathrm{z} \\
& 14^{* *} 7308 \mathrm{z} \\
& 14^{* *} 7308 \mathrm{z} \\
& 14^{* *} 7308 \mathrm{z} \\
& 14^{* *} 7308 \mathrm{z} \\
& 14^{* *}
\end{aligned}
$$

5307z 499534-17 86z 347316-17 86z 288215-17 86z 265292-17 86z 232955-17 86z 192127-17 86z 153919-17 86z 118889-17 86z 945994-18 86z 795899-18 $86 z$ 651073-18 86z 503065-18 86z 371516-18 86z 297703-18 86z 260989-18 86z 22277-17 86z 18687-17 86z 180791-18 86z 315902-18 86z 623068-18 86z $135875-1786 z$ 453767-17 86z 176486-16 86z $14 * * 7308 \mathrm{z}$ $14 * * 7308 z$ $14 * * 7308 \mathrm{z}$ $14 * 7308 \mathrm{z}$ $14 * * 7308 \mathrm{z}$ $14 * *$

5307z 302694-16 86z 19858-15 86z 159274-16 86z 144311-16 86z 123302-16 $86 z$ 971831-17 86z 734075-17 86z 526143-17 86z 392195-17 86z 319547-17 $86 z$ 262264-17 86z 228113-17 86z 226636-17 86z 246407-17 86z 270773-17 $86 z$ 321334-17 86z 432635-17 86z 866639-17 86z 208512-16 86z 18293-15 86z $164223-1686 z$ 467788-16 86z 919543-16 86z $14 * * 7308 \mathrm{z}$ $14 * * 7308 z$ $14 * * 7308 \mathrm{z}$ $14 * * 7308 z$ $14 * * 7308 z$

Figure E.8 Continued. 
RTFLUM - Strip DORT Scalar Fluxes to p0 Component $1 \$ \$ 21220-10 /$ ntrtf,ntfog,iedit.mm,iscat

1200000 / nbuf,intype,iotype,spare,spare

e $t$

$t$

Figure E.9 Card Input Data for the RTFLUM Flux Conversion Utility Program. 
DORT - Loop Transport Carrier Calculation. Co-60 source

- VELM61 $61 \mathrm{n}-23 \mathrm{~g}, \mathrm{P}-5$, endf/b-v, $\mathrm{mm}=240$ (sym)

61\$\$ 0214023 /ntflx,ntfog,ntsig,ntbsi.ntdsi

$000000 /$ ntfci,ntibi.ntibo,ntnpr,ntdir $24 \mathrm{e}$

/ ntdso

$62 \$ \$ 05753259$ / iadj,isctm,izm,im,jm

8434870 / igm,iht.ihs,ihm,mixl

$054540240 /$ mmesh,mtp,mtm,idfac,mm

11000 / ingeom,ibl,ibr,ibb,ibt

1 -1040 / isrmx, ifxmi,ifxmf,mode.ktype

20003 / / iacc,kalf,igtype,inpfxm,inpsrm

$\begin{array}{llllll}0 & 0 & 0 & 0 & -4 & -4 \\ \text { njntsr, nintsr, njntfx, nintfx, iact }\end{array}$

40110 / ired,ipdb2,ifxprt,icsprt,idirf

00120110 / jdirf,jdirl,nbuf,iepsbz,minblk

01111 / maxblk,isbt,msbt,msdm,ibfscl

450104 / intscl,itmscl,nofis,ifdb2z,iswp

2181000 / keyjn,keyin,nsigtp,norpos,normat

011500 -10/mstmax,negfix,locobj,lcmobj,nkeyfx

461000 / ncndin, neut,itally, isp1, isp2

e

$63^{* *} 0.07 .4+10$ 1-4 1-3 1-3 / tmax,xnf,eps,epp,epv

1-3 1.0 0.2 1.5 10.0 /epf,ekobj,evth,evchm,evmax

1.0 $1.0-1.00 .3 \quad 10.0$ /evkmx,evi,devdki,evdelk,sormin

1.0 1-4 1-2 0.0 - 1.5 /conacc,conscl,coneps,wsolmn,wsolii

$1.50 .60 .01-300.0$ /wsolcn,orf,fsnacc,flxmin,smooth

1-20.20.9/ epo,extrcv, theta

e $t$

$\mathrm{t}$

$81 * * \quad$ / wts mm240

$02 \mathrm{r} 102900-802 \mathrm{r} 307825-802 \mathrm{r} 510200-802 \mathrm{r} 708425-80$

2r901350-8 () 563869-8 316131-8 n2 $0641385-8$ 359590-8 n2 0

714976-8 400849-8 n2 0 784547-8 439853-8 n2 0 857529-8

480771-8 n2 0 642875-8 293289-8 479164-8 n3 0 681415-8

310872-8 507890-8 n3 0 716550-8 326901-8 534077-8 n3 0

745915-8 340298-8 555965-8 n3 0 775565-8 353825-8 578064-8

n3 0 489468-8 386282-8 513536-8 364389-8 n4 0 500102-8

394674-8 524693-8 372306-8 n4 0 508580-8 401365-8 533587-8

$378617-8$ n4 0 515474-8 406806-8 540820-8 383750-8 n4 0

517107-8 408094-8 542534-8 384965-8 n4 q 120

$82 * *$ / mus mm240

$-641230-7-421582-7 \mathrm{ml}-142963-6-939923-7 \mathrm{ml}-229252-6$

$-150724-6 \mathrm{ml}-315291-6-207291-6 \mathrm{ml}-399349-6-262555-6 \mathrm{ml}$

$-472796-6-411087-6-143488-6 \mathrm{~m} 2-537046-6-466952-6$

$-162988-6 \mathrm{~m} 2-598374-6-520275-6-181600-6 \mathrm{~m} 2-656401-6$

$-570729-6-199211-6 \mathrm{~m} 2-711034-6-618231-6-215791-6 \mathrm{~m} 2$

$-761567-6-713133-6-470428-6-164201-6 \mathrm{~m} 3-807567-6$

$-756207-6-498843-6-174119-6 \mathrm{~m} 3-849108-6-795106-6$

$-524503-6-183075-6$ m3 -885925-6 -829582-6 -547246-6

$-191013-6 \mathrm{~m} 3-917890-6-859514-6-566991-6-197905-6 \mathrm{~m} 3$

Figure E.10 Card Input Data for the DORT Calculation of the ${ }^{60} \mathrm{Co}$ Source Packaged in the Loop Transport Carrier SNF Shipping Cask. 
$-944812-6-922954-6-765692-6-505099-6-176303-6 \mathrm{~m} 4$

$-966490-6-944130-6-783260-6-516688-6-180348-6 \mathrm{~m} 4$

$-982847-6-960108-6-796516-6-525433-6-183400-6 \mathrm{~m} 4$

$-993815-6-970823-6-805405-6-531297-6-185447-6 \mathrm{~m} 4$

$-999313-6-976194-6-809860-6-534236-6-186473-6$ m4 q 120

$83 * *$ / etas mm240

$3 r-.9979423 r-.9897283 r-.9733673 r-.9489953 r-.916799$

$5 r-.8811725 r-.843553$ 5r-.801217 5r-.754412 5r-.703158

$7 \mathrm{r}-.6480867 \mathrm{r}-.5897767 \mathrm{r}-.5282227 \mathrm{r}-.4638287 \mathrm{r}-.396835$

9r-.3276139r-.256704 9r-.184425 9r-.111045 9r-.037054 g120

$84 \$ \$ 1234323$ / reg nos by zone

$\mathrm{t}$

$1 * *$ fo / fission spectrum

2** /axii $(\mathrm{jm}+1)$

4i0.0 3i3.81 1i6.35 8i7.30 16i13.98 2i28.59 30.81 1i31.45

$167 \mathrm{i} 33.354 \mathrm{i} 200.99205 .43206 .0711 \mathrm{i} 206.718 \mathrm{i} 218.132 \mathrm{i} 226.07$

1i228.30 1i229.25 1i230.529i231.52 238.45 239.09

$4 * * \quad /$ radii $(\mathrm{im}+1)$

2i0.0 lil $.334 \mathrm{i} 2.415 .083 \mathrm{i} 5.407 .303 \mathrm{i} 7.621 \mathrm{i} 9.361 \mathrm{i} 10.16$

$10.805 \mathrm{i} 11.121 \mathrm{i} 14.291 \mathrm{i} 15.2414 \mathrm{i} 16.832 \mathrm{i} 28.7330 .48$

$5^{* *} \mathrm{f} 1$ / energy group boundaries

$8 \$ \$$ / zone numbers by interval

zone 1-air, 2-ss347, 3-lead, 4-sae 1020

35r2 18r1 4q53/j-ints 1-5

$33 r 2$ 20r4 3q53 /j-ints 6-9

20r2 33r4 lq53 /j-ints 10-11

$24 \mathrm{r} 2$ 26r3 3r4 8q53/j-ints $12-20$

$15 r 5$ 9r2 26r3 3r4 16q53/j-ints 21-37

24r2 26r3 3r4 2q53/j-ints 38-40

10r1 14r2 26r3 3r4/j-int 41

10r1 6r2 34r3 3r4 lq53 /j-ints 42-43

15r1 1r2 34r3 3r4 167q53/j-ints 44-211

10r1 6r2 34r3 3r4 4q53/j-ints 212-216

10r1 12r2 28r3 3r4/j-int 217

$3 \mathrm{r} 6$ 19r2 28r3 3r4 /j-int 218

3r6 17r7 2r2 28r3 3r4 11q53/j-ints 219-230

5r6 15r7 2r2 28r3 3r4 8q53/j-ints 231-239

5r6 15r7 11r2 19r3 3r4 2q53 /j-ints 240-242

5r6 15r7 11r2 22r4 lq53 /j-ints 243-244

5r6 26r2 22r1 lq53/j-ints 245-246

11 r6 13r1 7r2 22r1 lq53/j-ints 247-248

24r1 1r2 28r1 9q53/j-ints 249-258

25r2 28r1 /j-int 259

$9 \$ \$ 17131913713 /$ mat by zone

$24 * *$ fl / importance by zone

$25 \$ \$-31-37-43-49 /$ mats for activity calcs

$26 \$ \$ 1 \quad 1 \quad 1 \quad 1 / \mathrm{x}$-sec positions for activity calcs

$27 * * 1.01 .01 .01 .0$ / activity multipliers

$28 \$ \$ 61$ r0 23r25 / inners by grp

Figure E.10 Continued. 
$29 \$ \$ 11639127218244246248248 /$ j-pos key fluxes $30 \$ \$ 127415353534113127$ / i-pos key fluxes

$96 * *{ }^{\mathrm{t}} 15 \mathrm{r} 1.038 \mathrm{r} 0.0 \quad / \mathrm{fi}(\mathrm{i}), \mathrm{i}=1, \mathrm{im}$

$97 * * \stackrel{t}{4} 43 \mathrm{r} 0.0168 \mathrm{r} 1.048 \mathrm{r} 0.0 / \mathrm{fj}(\mathrm{j}), \mathrm{j}=1, \mathrm{jm}$

$98 * * \stackrel{\mathrm{t}}{6} 61 \mathrm{r} 0.011 \mathrm{r} 0.01 .011 \mathrm{r} 0.0 / \mathrm{fg}(\mathrm{g}), \mathrm{g}=1, \mathrm{igm}$ $\mathrm{t}$

Figure E.10 Continued.

E-27 
***** DORT Input Changes for 25GWD/MTU LWR MFP Source ***** DORT - Loop Transport Carrier Calculation, MFP source: $25 \mathrm{GWD} / \mathrm{MTu}$ 63**0.0 1.39+10 1-4 1-3 1-3 / tmax.xnf,eps,epp,epv

$98 * * 61 \mathrm{r} 0.0 / \mathrm{fg}(\mathrm{g}), \mathrm{g}=1 . \mathrm{igm}$

8.19-03 1.73-()1 1.46-01 2.53-01 1.18+00 3.49+00 1.04+(01 8.08+01

$1.93+032.25+046.36+061.59+082.52+086.96+095.85+079.24+(07$

$1.41+084.43+085.22+085.16+088.70+082.15+091.72+09$

***** DORT Input Changes for 50GWD/MTU LWR MFP Source ***** DORT - Loop Transport Carrier Calculation, MFP source; 50GWD/MTu 63**0.0 1.47+101-41-3 1-3 / tmax,xnf,eps,epp,epv $98 * * 61 \mathrm{r} 0.0 / \mathrm{fg}(\mathrm{g}), \mathrm{g}=1, \mathrm{igm}$

3.10-02 6.53-01 5.53-01 9.54-01 4.44+00 1.32+01 3.91+01 1.73+02 $4.33+032.37+048.85+062.28+083.71+087.23+098.13+079.71+07$ $1.46+084.66+085.95+085.45+088.81+082.26+091.81+09$

***** DORT Input Changes for Cs-137 Source ***** DORT - Loop Transport Carrier Calculation, Cs-137 source 63** 0.0 3.467+10 1-4 1-3 1-3 / tmax,xnf,eps,epp,epv $98 * * 61 \mathrm{r} 0.013 \mathrm{r} 0.01 .09 \mathrm{r} 0.0 / \mathrm{fg}(\mathrm{g}), \mathrm{g}=1, \mathrm{igm}$

***** DORT Input Changes for ORR MFP Source; 20\% U235***** DORT - Loop Transport Carrier Calculation, ORR MFP Source; 20\% U235 $63 * * 0.01 .71+101-41-31-3$ / tmax,xnf,eps,epp,epv $98 * * 61 \mathrm{r} 0.0 / \mathrm{fg}(\mathrm{g}), \mathrm{g}=1$, igm

4.52-05 9.59-04 8.15-04 1.41-03 6.60-03 1.98-02 5.93-02 2.85+01

$3.75+023.57+043.07+066.03+071.29+087.86+094.62+071.36+08$

$2.23+086.67+086.48+087.68+081.13+092.92+092.48+09$

***** DORT Input Changes for ORR MFP Source; 40\% U235***** DORT - Loop Transport Carrier Calculation, ORR MFP Source; 40\% U235 $63 * * 0.01 .78+101-41-31-3$ / tmax.xnf,eps,epp,epv $98 * * 61 \mathrm{r} 0.0 / \mathrm{fg}(\mathrm{g}), \mathrm{g}=1, \mathrm{igm}$

$4.91-021.04+(008.78-011.51+007.04+(0) 2.09+016.20+012.26+() 2$ $1.41+043.27+(048.35+062.20+085.37+088.06+(091.35+081.33+(08$ $2.07+086.40+087.33+087.37+081.07+092.86+092.46+09$

***** DORT Input Changes for ORR MFP Source; 93\% U235***** DORT - Loop Transport Carrier Calculation, ORR MFP Source; 93\% U235 $63 * * 0.01 .83+101-41-31-3$ / tmax,xnf,eps,epp,epv $98 * * 61 \mathrm{r} 0.0 / \mathrm{fg}(\mathrm{g}), \mathrm{g}=1, \mathrm{igm}$

1.53-03 3.23-02 2.74-02 4.72-02 2.20-01 6.55-01 1.95+00 2.69+01 $8.68+033.43+048.45+062.18+085.09+088.03+091.28+081.43+08$ $2.25+086.92+087.78+087.93+081.15+093.02+092.57+09$

Figure E.11 Card Input Data Changes for the DORT Calculation of the Other Sources Packaged in the Loop Transport Carrier SNF Shipping Cask. 


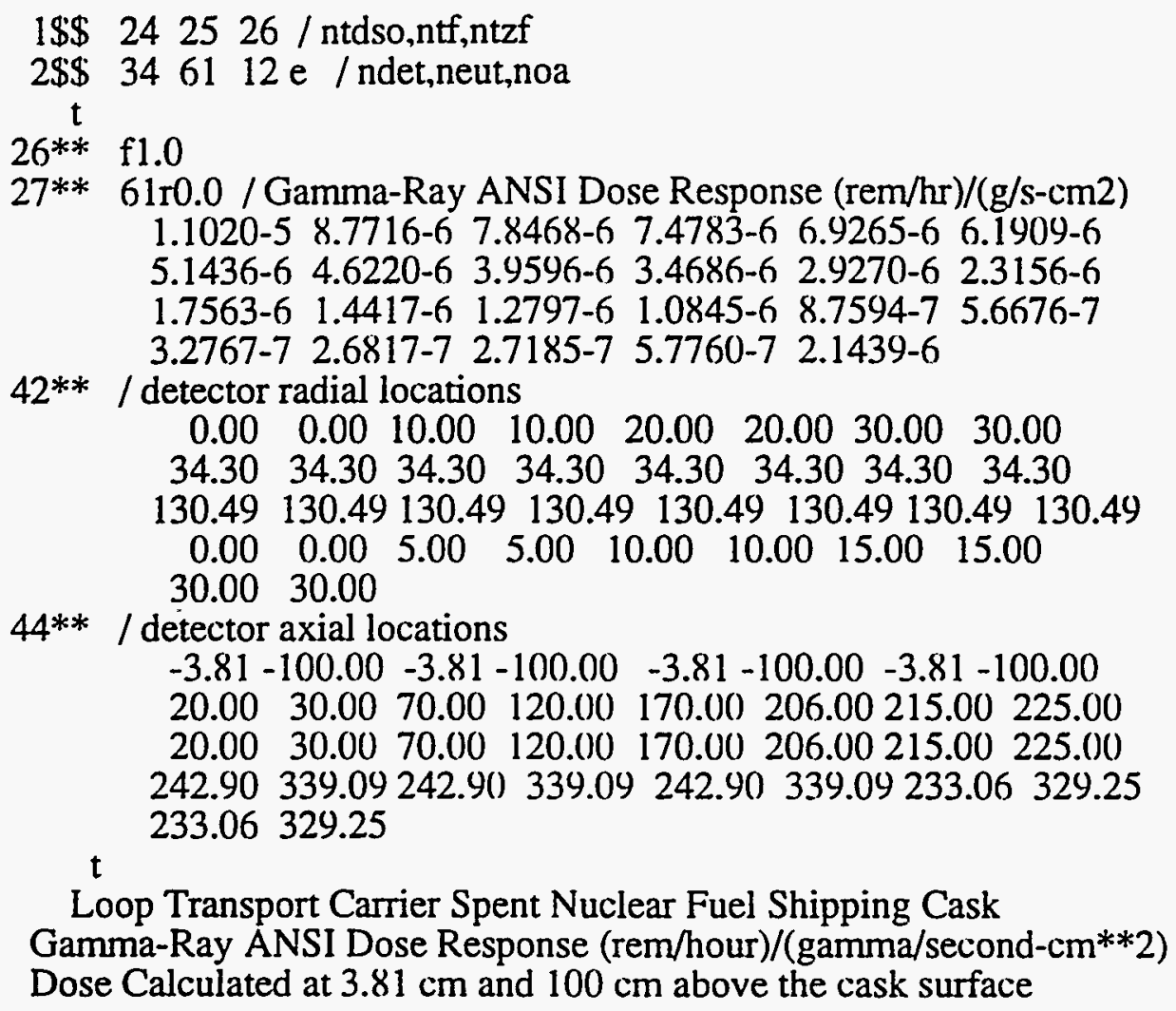

Figure E.12 Card Input Data for the FAISTF Calculations of the Surface and One Meter Distance Dose Rates at the Detector Locations Used in the Loop Transport Carrier SNF Shipping Cask Shielding Analysis. 
Loop Transport Carrier DORT Geometry Model

$0 \$ \$ 2210 \mathrm{e} /$ nflsv,irflx,irfly

$1 \$ \$ 53259700$ / imx,jm,izm,imap,itip

842540010 / igm,nlvmx,nbuf,igeom,ivol

00000 / ishd,irsp,ihm.nmat.igx

0 e / ilvck

2** 0.08 .511 .01 .01 .0 / size,xpage,ypage,xscl.yscl

$0.00 .00 .00 .00 .0 / x s t p, y s t p, x m i n, x \max , y \min$

0.00 .25 e / ymax,eps

$3 \$ \$ 53 \mathrm{fO}$

$4 \$ \$$ f1 t

$5^{* *}$ /axii $(\mathrm{jm}+1)$

$4 \mathrm{i} 0.03 \mathrm{i} 3.811 \mathrm{i} 6.358 \mathrm{i} 7.3016 \mathrm{i} 13.982 \mathrm{i} 28.5930 .811 \mathrm{i} 31.45$

167i33.35 4i200.99 205.43 206.07 11i206.71 8i218.13 2i226.07

1i228.30 1i229.25 1i230.52 9i231.52 238.45 239.09

6** /radii (im+1)

2i0.0 li1.33 4i2.41 5.08 3i5.40 7.30 3i7.62 1i9.36 li10.16

$10.805 \mathrm{i} 11.12$ li14.29 $1 \mathrm{i} 15.2414 \mathrm{i} 16.832 \mathrm{i} 28.7330 .48$

$8 \$ \$$ / zone numbers by interval

$35 \mathrm{r} 218 \mathrm{r} 14 \mathrm{q} 53$ /j-ints $1-5$

$33 \mathrm{r} 2$ 20r4 3q53 /j-ints $6-9$

20r2 33r4 lq53 /j-ints 10-11

24r2 26r3 3r4 8q53 /j-ints $12-20$

$15 \mathrm{r} 5$ 9r2 26r3 3r4 16q53 /j-ints 21-37

$24 \mathrm{r} 2$ 26r3 3r4 2q53/j-ints 38-40

10r1 14r2 26r3 3r4/j-int 41

10r1 6r2 34r3 3r4 lq53/j-ints 42-43

15r1 1r2 34r3 3r4 167q53 /j-ints 44-211

$10 \mathrm{r} 16 \mathrm{r} 234 \mathrm{r} 33 \mathrm{r} 4 \mathrm{4q} 53$ /j-ints 212-216

10r1 12r2 28r3 3r4/j-int 217

3r6 19r2 28r3 3r4 /j-int 218

3r6 17r7 2r2 28r3 3r4 11 lq53 /j-ints 219-230

5r6 15r7 2r2 28r3 3r4 8q53/j-ints 231-239

5r6 15r7 1 1r2 19r3 3r4 2q53 /j-ints 240-242

5r6 15r7 11r2 22r4 lq53 /j-ints 243-244

5r6 26r2 22rl lq53/j-ints 245-246

11 r6 13r1 7r2 22r1 lq53/j-ints 247-248

$24 \mathrm{r} 1 \mathrm{rr} 28 \mathrm{r} 19 \mathrm{q} 53$ /j-ints 249-258

25r2 28r1 /j-int 259

$9 \$ \$ 17131913713 /$ mat by zone

$t$

Radius (cm)\$

Height $(\mathrm{cm}) \$$

Loop Transport Carrier Gamma-Ray Dose Rate\$

$13 \$ \$ 6284100120$ / iga,igb,nlvl,iplt,ipos

$00 \mathrm{e} /$ imat,ichk

$14 * * 1.0-1.0-1.0$ e t / fac,fmin,fmax

Figure E.13. Card Input Data for the ISOPLOT Isodose Contour Maps for the Loop Transport Carrier SNF Shipping Cask Shielding Analysis. 
15** $61 \mathrm{r} 0.0$ / Gamma-Ray ANSI Dose Response (rem/hr)/(g/s.cm2) 1.1020-5 8.7716-6 7.8468-6 7.4783-6 6.9265-6 6.1909-6 5.1436-6 4.6220-6 3.9596-6 3.4686-6 2.9270-6 2.3156-6 $\begin{array}{llllll}1.7563-6 & 1.4417-6 & 1.2797-6 & 1.0845-6 & 8.7594-7 & 5.6676-7\end{array}$ $\begin{array}{lllll}3.2767-7 & 2.6817-7 & 2.7185-7 & 5.7760)-7 & 2.1439-6\end{array}$

last

Loop Transport Carrier DORT Geometry Model

$1 \$ \$ 53259720$ / imx,jm,izm,imap,itip

842540010 / igm,nlvmx,nbuf,igeom,ivol

00000 / ishd,irsp,ihm,nmat,igx

$0 \mathrm{e} /$ ilvck

$2 * * 0.08 .511 .01 .01 .0$ / size,xpage,ypage,xscl,yscl

$0.00 .00 .020 .00 .0 / x s t p, y s t p, x \min , x \max , y \min$

35.00 .25 e / ymax,eps

$\mathrm{t}$

Radius (cm)\$

Height (cm)\$

Loop Transport Carrier Door Plug Gamma-Ray Dose Rate\$

$13 \$ \$ 6284100120$ / iga,igb,nlvl,iplt,ipos

$00 \mathrm{e} /$ imatichk

$14 * * 1.0-1.0-1.0$ e $\mathrm{t} / \mathrm{fac}$,fmin,fmax

$15^{* *} \quad 61 \mathrm{r0} .0$ / Gamma-Ray ANSI Dose Response (rem/hr)/(g/s.cm2) 1.1020-5 8.7716-6 7.8468-6 7.4783-6 6.9265-6 6.1909-6 5.1436-6 $4.6220-6 \quad 3.9596-6 \quad 3.4686-6 \quad 2.9270-6 \quad 2.3156-6$ $\begin{array}{lllllll}1.7563-6 & 1.4417-6 & 1.2797-6 & 1.0845-6 & 8.7594-7 & 5.6676-7\end{array}$ $\begin{array}{llllll}3.2767-7 & 2.6817-7 & 2.7185-7 & 5.7760-7 & 2.1439-6\end{array}$

last

Loop Transport Carrier DORT Geometry Model

$1 \$ \$ 53259720 /$ imx,jm,izm,imap,itip

842540010 / igm,nlvmx,nbuf,igeom,ivol

$00000 /$ ishd,irsp,ihm,nmat,igx

$0 \mathrm{e} /$ ilvck

2** 0.08 .511 .01 .01 .0 / size,xpage,ypage,xscl,yscl

$0.00 .00 .015 .0200 .0 / x s t p, y s t p, x \min , x \max , y \min$

240.00 .25 e / ymax.eps

$\mathrm{t}$

Radius (cm)\$

Height $(\mathrm{cm}) \$$

Loop Transport Carrier End Plug Gamma-Ray Dose Rate\$

$13 \$ \$ 6284100120$ / iga,igb,nlvl,iplt,ipos

$00 \mathrm{e} /$ imat,ichk

$14 * * 1.0-1.0-1.0$ e t / fac,fmin,fmax

15** $61 \mathrm{r0} 0.0$ / Gamma-Ray ANSI Dose Response (rem $/ \mathrm{hr}) /(\mathrm{g} / \mathrm{s} . \mathrm{cm} 2)$ 1.1020-5 8.7716-6 7.8468-6 7.4783-6 6.9265-6 6.1909-6 5.1436-6 $4.6220-6 \quad 3.9596-6 \quad 3.4686-6 \quad 2.9270-6 \quad 2.3156-6$ $\begin{array}{lllllll}1.7563-6 & 1.4417-6 & 1.2797-6 & 1.0845-6 & 8.7594-7 & 5.6676-7\end{array}$ $\begin{array}{llllll}3.2767-7 & 2.6817-7 & 2.7185-7 & 5.7760-7 & 2.1439-6\end{array}$

$t$

last

Figure E.13 Continued. 
DORT - In-Pile Loop LITR HB-2 Carrier Calculation, Co-60 source

' VELM61 61n-23g, P-5, endf/b-v, mm = 240 (sym)

$61 \$ \$ 0214023 / n t f l x, n t f o g, n t s i g, n t b s i, n t d s i$

$00000 / \mathrm{ntfci}, n t i b i, n t i b o, n t n p r, n t d i r$ $24 \mathrm{e}$ / ntdso

$62 \$ \$ 051043385$ / iadj,isctm.izm.im.jm

8434870 / igm,iht,ihs, ihm,mixl

054540240 / mmesh.mtp.mtm.idfac,mm

11000 / ingeom,ibl,ibr,ibb,ibt

$1-1040$ / isrmx, ifxmi,ifxmf, mode,ktype

20003 / iacc,kalf,igtype,inpfxm,inpsrm

$0000-4$ / njntsr,nintsr, njntfx.nintfx, iact

30110 / ired,ipdb2,ifxprt,icsprt,idirf

00120110 / jdirf,jdirl,nbuf,iepsbz,minblk

$01111 / \mathrm{maxblk}$,isbt,msbt,msdm,ibfscl

450104 / intscl,itmscl,nofis,ifdb2z,iswp

3571000 / keyjn,keyin,nsigtp,norpos,normat

$0115000-10$ / mstmax.negfix,locobj,lcrnobj,nkeyfx

461000 / nondin,neut.itally,isp1,isp2

$\mathrm{e}$

$63 * * 0.07 .4+101-41-31-3$ / tmax,xnf,eps,epp,epv

1-3 1.0 0.21 .510 .0 /epf,ekobj,evth,evchm,evmax

1.0 1.0 - 1.00 .310 .0 /evkmx,evi,devdki,evdelk,sormin

1.0 1-4 1-2 $0.0-1.5$ /conacc,conscl,coneps, wsolmn,wsolii

$1.50 .60 .01-300.0$ /wsolcn,orf,fsnacc,flxmin,smooth

$1-20.20 .9$ / epo,extrcv, theta

e $t$

$81 * *^{\mathrm{t}} / \mathrm{wts} \mathrm{mm} 240$

02 r 102900-8 02 2r307825-8 0 2r510200-8 02 2r708425-8 0

2 r901350-8 () 563869-8 316131-8 n2 0 641385-8 359590-8 n2 0

714976-8 400849-8 n2 0 784547-8 439853-8 n2 0 857529-8

480771-8 n2 0 642875-8 293289-8 479164-8 n3 0 681415-8

310872-8 507890-8 n3 0 716550-8 326901-8 534077-8 n3 0

745915-8 340298-8 555965-8 n3 0 775565-8 353825-8 578064-8

n3 $0489468-8386282-8513536-8364389-8$ n4 $0500102-8$

394674-8 524693-8 372306-8 n4 0 508580-8 401365-8 533587-8

378617-8 n4 0 515474-8 406806-8 540820-8 383750-8 n4 0

517107-8 408094-8 542534-8 384965-8 n4 q120

$82 * *$ / mus mm240

$-641230-7-421582-7 \mathrm{ml}-142963-6-939923-7 \mathrm{ml}-229252-6$

$-150724-6 \mathrm{ml}-315291-6-207291-6 \mathrm{ml}-399349-6-262555-6 \mathrm{ml}$

$-472796-6-411087-6-143488-6 \mathrm{~m} 2-537046-6-466952-6$

$-162988-6 \mathrm{~m} 2-598374-6-520275-6-181600-6 \mathrm{~m} 2-656401-6$

$-570729-6-199211-6 \mathrm{~m} 2-711034-6-618231-6-215791-6 \mathrm{~m} 2$

$-761567-6-713133-6-470428-6-164201-6 \mathrm{~m} 3-807567-6$

$-756207-6-498843-6-174119-6 \mathrm{~m} 3-849108-6-795106-6$

$-524503-6-183075-6 \mathrm{~m} 3-885925-6-829582-6-547246-6$

Figure E.14 Card Input Data for the DORT Calculation of the

${ }^{60} \mathrm{Co}$ Source Packaged in the In-Pile Loop LITR HB-2

Carrier SNF Shipping Cask. 
$-191013-6$ m3 - $917890-6-859514-6-566991-6-197905-6 m 3$ $-944812-6-922954-6-765692-6-505099-6-176303-6 \mathrm{~m} 4$ $-966490-6-944130-6-783260-6-516688-6-180348-6 \mathrm{~m} 4$ $-982847-6-960108-6-796516-6-525433-6-183400-6 \mathrm{~m} 4$ $-993815-6-970823-6-805405-6-531297-6-185447-6 \mathrm{~m} 4$ $-999313-6-976194-6-809860-6-534236-6-186473-6$ m4 q120 $83 * *$ / etas mm240

$3 \mathrm{r}-.9979423 \mathrm{r}-.9897283 \mathrm{r}-.9733673 \mathrm{r}-.9489953 \mathrm{r}-.916799$

$5 r-.8811725 r-.843553$ 5r-.801217 5r-.754412 5r-.703158

7r-.648086 7r-.589776 7r-.528222 7r-.463828 7r-.396835

$9 \mathrm{r}-.3276139 \mathrm{r}-.2567049 \mathrm{r}-.1844259 \mathrm{r}-.1110459 \mathrm{r}-.037054 \mathrm{~g} 120$

$84 \$ \$ 1233322222$ / reg nos by zone

$t$

$1 * *$ fo / fission spectrum

$2 * *$ /axii $(\mathrm{jm}+1)$

li0.0 1.27 1.91 23i2.55 17.79 18.43 324i 19.07262 .91263 .55

23i264.19 279.43280.07 1i280.71281.98

$4 * *$ /radii $(\mathrm{im}+1)$

$16 \mathrm{i} 0.010 .6410 .9511 .431 \mathrm{i} 12.0619 \mathrm{i} 13.3426 .1926 .83$

$5 * *$ f1 / energy group boundaries

$8 \$ \$$ / zone numbers by interval

' zone 1-air, 2-ss347, 3-lead

19r1 23r2 1r6 lq43 /j-ints $1-2$

$18 \mathrm{r} 14 \mathrm{r} 2$ 20r3 lr6 /j-int 3

22r2 20r3 lr6/j-int 4

20r4 2r7 20r3 1r6 23q43/j-ints 5-28

22r9 20r3 1r6/j-int 29

17r1 5r9 20r3 1r6/j-int 30

17r1 1r9 24r3 1r6 324q43 /j-ints 31-355

17r1 5r9 20r3 1r6 /j-int 356

22r9 20r3 ir6/j-int 357

20r5 2r8 20r3 lr6 23q43/j-ints 358-381

22r10 20r3 lr6/j-int 382

18 r1 4r10 20r3 lr6 /j-int 383

19r1 23r10 lr6 lq43/j-ints 384-385

9\$\$1713131377777/mat by zone

$24 * *$ fl / importance by zone

$25 \$ \$-31-37-43-49 /$ mats for activity calcs

$26 \$ \$ 1111 / \mathrm{x}$-sec positions for activity calcs

$27 * * 1.01 .01 .01 .0$ / activity multipliers

$28 \$ \$ 61 r 023 r 25$ / inners by grp

29\$\$ 11111442984138193 / j-pos key fluxes

$30 \$ \$ 2128344211043434343$ /i-pos key fluxes

$96 * *{ }^{\mathrm{t}} 17 \mathrm{r} 1.026 \mathrm{r} 0.0 \quad$ /fi(i), $\mathrm{i}=1, \mathrm{im}$ $\mathrm{t}$

$97 * * 29 \mathrm{r} 0.0327 \mathrm{rl} .029 \mathrm{r} 0.0 / \mathrm{fj}(\mathrm{j}), \mathrm{j}=1, \mathrm{jm}$ $\mathrm{t}$

$98 * * 61 \mathrm{r0} 011 \mathrm{r} 0.01 .011 \mathrm{r0} 0.0 / \mathrm{fg}(\mathrm{g}), \mathrm{g}=1$, igm

$\mathrm{t}$

Figure E.14 Continued. 
----- Input Changes for 25GWD/MTU LWR MFP Source -----

DORT - In-Pile Loop LITR HB-2 Carrier Calculation. MFP source: 25GWD/MTu $63 * * 0.01 .39+101-41-31-3$ / tmax,xnf,eps,epp,epv

$98 * * 61 \mathrm{r} 0.0$ / fg(g), g=1,igm

$8.19-03$ 1.73-01 $1.46-012.53-011.18+003.49+(001.04+018.08+(01$

$1.93+032.25+046.36+061.59+082.52+(1) 86.96+095.85+079.24+107$

$1.41+084.43+085.22+085.16+088.70+082.15+091.72+09$

-.-- Input Changes for 50GWD/MTU LWR MFP Source --..-

DORT - In-Pile Loop LITR HB-2 Carrier Calculation. MFP source; 50GWD/MTu $63 * * 0.01 .47+101-41-31-3$ / tmax,xnf,eps,epp,epv

$98 * * 61 \mathrm{r} 0.0 / \mathrm{fg}(\mathrm{g}), \mathrm{g}=1$,igm

3.10-02 6.53-01 5.53-01 9.54-01 4.44+00 1.32+01 3.91+01 1.73+(1)2

$4.33+032.37+048.85+062.28+083.71+087.23+098.13+079.71+(17$

$1.46+084.66+085.95+085.45+088.81+082.26+091.81+09$

-.--- Input Changes for Cs-137 Source -----

DORT - In-Pile Loop LITR HB-2 Carrier Calculation. Cs-137 source $63 * * 0.03 .467+101-41-31-3$ / tmax,xnf,eps,epp,epv

98** 61r0.0 13r0.0 1.09r0.0 / fg(g), g=1,igm

---- Input Changes for ORR MFP Source; 20\% U235 -----

DORT - In-Pile Loop LITR HB-2 Carrier Calculation, ORR MFP Source: 20\% U235 $63 * * 0.01 .71+101-41-31-3$ / tmax,xnf,eps,epp,epv

$98 * 61 \mathrm{r} 0.0 / \mathrm{fg}(\mathrm{g}), \mathrm{g}=1, \mathrm{igm}$

4.52-05 9.59-04 8.15-04 1.41-03 6.60-03 1.98-02 5.93-02 2.85+1)1

$3.75+023.57+043.07+066.03+071.29+087.86+094.62+071.36+08$

$2.23+086.67+086.48+087.68+081.13+092.92+092.48+09$

---- Input Changes for ORR MFP Source; 40\% U235 -----

DORT - In-Pile Loop LITR HB-2 Carrier Calculation, ORR MFP Source; 40\% U235 $63 * * 0.01 .78+101-41-31-3$ / tmax,xnf,eps,epp,epv

$98 * * 61 \mathrm{r} 0.0 / \mathrm{fg}(\mathrm{g}), \mathrm{g}=1, \mathrm{igm}$

4.91-02 1.04+00 8.78-()1 1.51+(00 7.04+00 2.09+016.20+(01 2.26+(1)2

$1.41+043.27+(048.35+062.20+085.37+088.06+091.35+081.33+(08$

$2.07+086.40+087.33+087.37+081.07+092.86+092.46+09$

Input Changes for ORR MFP Source; 93\% U235 --..-

DORT - In-Pile Loop LITR HB-2 Carrier Calculation, ORR MFP Source; 93\% U235 $63 * * 0.01 .83+101-41-31-3$ / tmax,xnf,eps,epp,epv

$98 * * 61 \mathrm{r} 0.0 / \mathrm{fg}(\mathrm{g}), \mathrm{g}=1$, igm

1.53-03 3.23-02 2.74-02 4.72-02 2.20-01 6.55-01 1.95+00 2.69+01

$8.68+033.43+048.45+062.18+085.09+088.03+(091.28+081.43+(08$

$2.25+086.92+(087.78+087.93+081.15+093.02+092.57+09$

Figure E.15 Card Input Data Changes for the DORT Calculation of the Other Sources Packaged in the In-Pile Loop LITR HB-2 Carrier SNF Shipping Cask. 


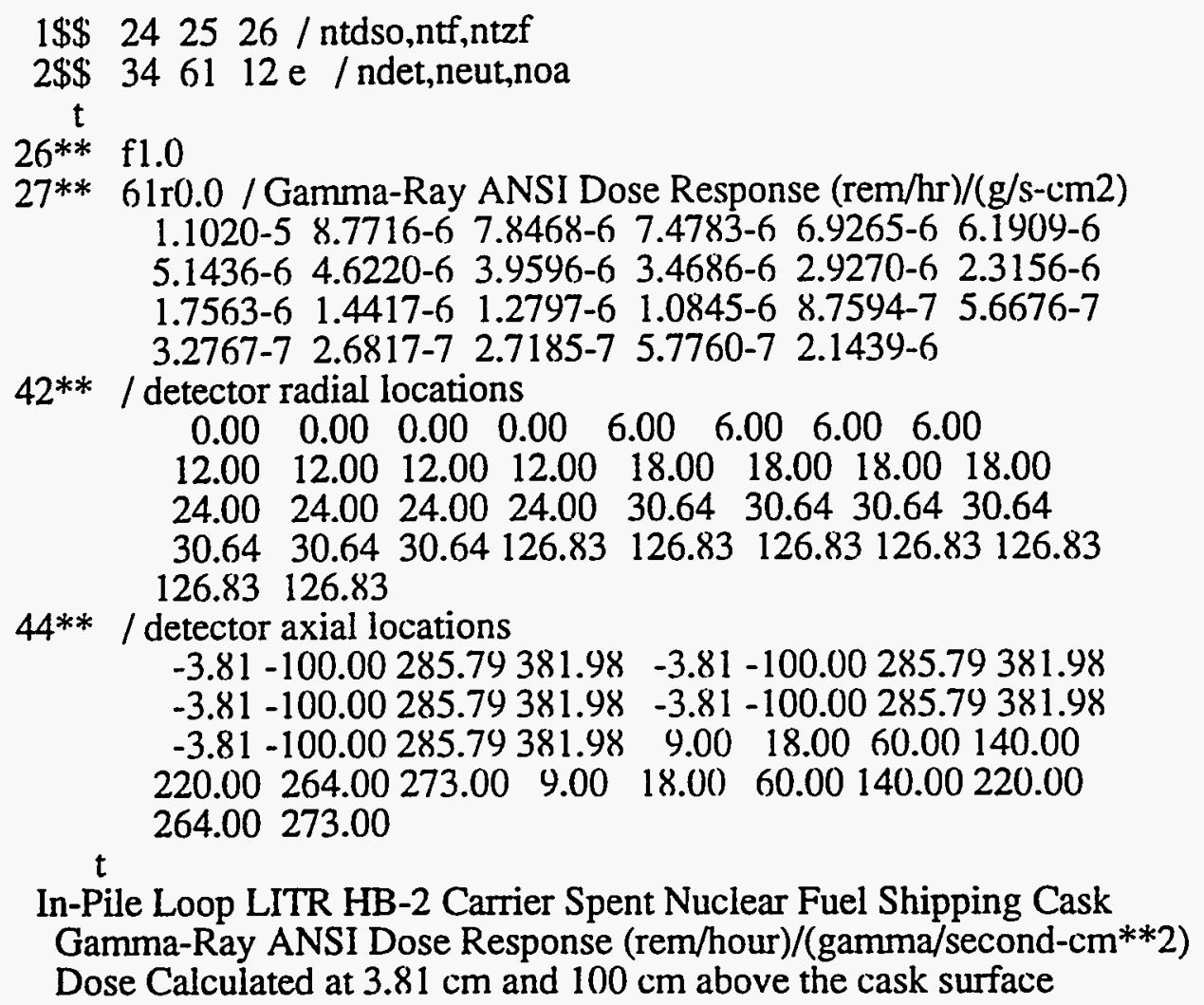

Figure E.16 Card Input Data for the FALSTF Calculations of the Surface and One Meter Distance Dose Rates at the Detector Locations Used in the In-Pile Loop LITR HB-2 Carrier SNF Shipping Cask Shielding Analysis. 
In-Pile Loop LITR HB-2 Carrier DORT Geometry Model

$0 \$ \$ 2210 \mathrm{e} /$ nflsv,irflx,irfly

$1 \$ \$ 433851000 /$ imx,jm,izm.imap,itip-

842540010 / igm,nlvmx,nbuf,igeom,ivol

00000 / ishd,irsp,ihm,nmat,igx

$0 \mathrm{e} /$ ilvck

2** 0.08 .511 .01 .01 .0 / size,xpage,ypage,xscl,yscl

0.00 .00 .00 .00 .0 / xstp,ystp.xmin.xmax,ymin

0.00 .25 e / ymax,eps

$$
\mathrm{t}
$$

$3 \$ \$ 43 \mathrm{fO}$

$4 \$ \$ \mathrm{fl}$

5** /axii $(\mathrm{jm}+1)$

$1 \mathrm{i} 0.01 .271 .9123 \mathrm{i} 2.5517 .7918 .43324 \mathrm{i} 19.07262 .91263 .55$

$23 \mathrm{i} 264.19279 .43280 .071 \mathrm{i} 280.71281 .98$

6** /radii (im+1)

$16 \mathrm{i} 0.010 .6410 .9511 .431 \mathrm{i} 12.0619 \mathrm{i} 13.3426 .1926 .83$

$8 \$ \$$ / zone numbers by interval

19r1 23r2 lr6 lq43 /j-ints 1-2

$18 \mathrm{rl} 4 \mathrm{r} 2$ 20r3 1r6 /j-int 3

22r2 20r3 lr6 /j-int 4

20r4 2r7 20r3 1r6 23a43 /j-ints 5-28

22r9 20r3 1r6 /j-int 29

17r1 5r9 20r3 lr6 /j-int 30

17r1 1r9 24r3 1r6 324q43/j-ints 31-355

17r1 5r9 20r3 1r6 /j-int 356

22r9 20r3 1r6/j-int 357

20r5 2r8 20r3 1r6 23q43/j-ints 358-381

22r10 20r3 1r6/j-int 382

18r1 4r10 20r3 1r6 /j-int 383

19r1 23r10 lr6 lq43/j-ints 384-385

$9 \$ \$ 1713131377777$ / mat by zone

$\mathrm{t}$

Radius (cm)\$

Height $(\mathrm{cm}) \$$

In-Pile Loop LITR HB-2 Carrier Gamma-Ray Dose Rate\$

$13 \$ \$ 6284100120$ / iga,igb,nlvl,iplt,ipos

$00 \mathrm{e} /$ imat,ichk

$14 * * 1.0-1.0-1.0 \mathrm{e} / \mathrm{fac}, \mathrm{fmin}, \mathrm{fmax}$

$\mathrm{t}$

15** 61r0.0 / Gamma-Ray ANSI Dose Response (rem/hr) $/(\mathrm{g} / \mathrm{s.cm} 2)$

1.1020-5 8.7716-6 7.8468-6 7.4783-6 $6.9265-6 \quad 6.1909-6$

$\begin{array}{llllll}5.1436-6 & 4.6220-6 & 3.9596-6 & 3.4686-6 & 2.9270-6 & 2.3156-6\end{array}$

$\begin{array}{lllllll}1.7563-6 & 1.4417-6 & 1.2797-6 & 1.0845-6 & 8.7594-7 & 5.6676-7\end{array}$

$\begin{array}{lllll}3.2767-7 & 2.6817-7 & 2.7185-7 & 5.7760-7 & 2.1439-6\end{array}$

$t$

last

Figure E.17 Card Input Data for the ISOPLOT Isodose Contour Maps for the In-Pile Loop LITR HB-2 Carrier SNF Shipping

Cask Shielding Analysis. 
In-Pile Loop LITR HB-2 Carrier DORT Geometry Model

$1 \$ \$ 433851020 / \mathrm{imx}$,jm,izm.imap,itip

842540010 / igm,nlvmx.nbuf.igeom.ivol

$00000 /$ ishd,irsp,ihm,nmat,igx

$0 \mathrm{e} /$ ilvck

2** 0.08 .511 .01 .01 .0 / size,xpage,ypage,xscl,yscl

$0.00 .00 .015 .0-2.0 / x s t p, y s t p, x \min , x \max , y \min$

22.00 .25 e / ymax,eps

$t$

Radius (cm)\$

Height $(\mathrm{cm}) \$$

LITR HB-2 Carrier Double Door Gamma-Ray Dose Rate\$

$13 \$ \$ 6284100120$ / iga,igb,nlvl.iplt,ipos

00 e / imat,ichk

$14 * * 1.0-1.0-1.0 \mathrm{e} / \mathrm{fac}, \mathrm{fmin}, \mathrm{fmax}$

$t$

$15^{* *} 61 \mathrm{r} 0.0$ / Gamma-Ray ANSI Dose Response (rem/hr)/(g/s.cm2)

1.1020-5 8.7716-6 7.8468-6 7.4783-6 $6.9265-6 \quad 6.1909-6$

$\begin{array}{cccccc}5.1436-6 & 4.6220-6 & 3.9596-6 & 3.4686-6 & 2.9270-6 & 2.3156-6\end{array}$

$\begin{array}{lllllll}1.7563-6 & 1.4417-6 & 1.2797-6 & 1.0845-6 & 8.7594-7 & 5.6676-7\end{array}$

$\begin{array}{llllll}3.2767-7 & 2.6817-7 & 2.7185-7 & 5.7760-7 & 2.1439-6\end{array}$

last

In-Pile Loop LITR HB-2 Carrier DORT Geometry Model

$1 \$ \$ 433851020$ / imx,jm,izm,imap,itip

842540010 / igm,nlvmx,nbuf,igeom,ivol

00000 / ishd,irsp,ihm,nmat,igx

$0 \mathrm{e} /$ ilvck

2** 0.08 .511 .01 .01 .0 / size,xpage,ypage,xscl,yscl

0.00 .00 .015 .0260 .0 / xstp,ystp,xmin,xmax,ymin

284.00 .25 e / ymax,eps

$\mathrm{t}$

Radius (cm)\$

Height (cm)\$

LITR HB-2 Carrier Large Door Gamma-Ray Dose Rate\$

$13 \$ \$ 6284100120$ / iga,igb,nlvl,iplt,ipos

$00 \mathrm{e} /$ imatichk

$14^{* *} 1.0-1.0-1.0 \mathrm{e} / \mathrm{fac}, \mathrm{fmin}, \mathrm{fmax}$

$\mathrm{t}$

$15 * * 61 \mathrm{r} 0.0$ / Gamma-Ray ANSI Dose Response (rem/hr)/(g/s.cm2)

1.1020-5 8.7716-6 7.8468-6 7.4783-6 $6.9265-6 \quad 6.1909-6$

5.1436-6 $4.6220-6 \quad 3.9596-6 \quad 3.4686-6 \quad 2.9270-6 \quad 2.3156-6$

$\begin{array}{lllllll}1.7563-6 & 1.4417-6 & 1.2797-6 & 1.0845-6 & 8.7594-7 & 5.6676-7\end{array}$

$\begin{array}{lllll}3.2767-7 & 2.6817-7 & 2.7185-7 & 5.7760-7 & 2.1439-6\end{array}$

$t$

last

Figure E-17. Continued. 
DORT - 6.5 inch HRLEL Carrier Calculation, Co-60 source

, VELM61 61n-23g, P-5, endf/b-v, mm = 240 (sym)

$61 \$ \$ 0214023$ / ntflx,ntfog,ntsig,ntbsi,ntdsi

$000000 /$ ntfci,ntibi,ntibo.ntnpr,ntdir

$24 \mathrm{e} /$ / ntdso

$62 \$ \$ 051246269$ / iadj,isctm.izm.im.jm

8434870 / igm,iht,ihs,ihm.mixl

054540240 / mmesh,mtp,mtm.idfac,mm

11000 / ingeom,ibl,ibr,ibb,ibt

$1-1040$ / isrmx, ifxmi,ifxmf,mode,ktype

20003 / iacc.kalf,igtype,inpfxm,inpsrm

$00000-4$ / njntsr,nintsr,njntfx,nintfx,iact

40110 / ired,ipdb2,ifxprt,icsprt,idirf

00120110 / jdirf,jdirl,nbuf,iepsbz,minblk

011111 / maxblk,isbt,msbt,msdm,ibfscl

450104 / intscl,itmscl,nofis,ifdb2z,iswp

4110000 / keyjn,keyin,nsigtp.norpos,normat

$0115000-12$ / mstmax, negfix,locobj,lcmobj,nkeyfx

461000 / ncndin,neut,itally,ispl,isp2

$\mathrm{e}$

63** 0.0 7.4+10 1-4 1-3 1-3 / tmax,xnf,eps.epp,epv

1-3 1.00.2 1.5 10.0 /epf,ekobj,evth,evchm,evmax

$1.01 .0-1.00 .310 .0$ /evkmx,evi,devdki,evdelk,sormin

$1.01-4$ 1-2 0.0 - 1.5 /conacc,conscl,coneps,wsolmn,wsolii

$1.50 .60 .01-300.0$ / wsolcn,orf,fsnacc,flxmin,smooth

1-2 0.20 .9 / epo,extrcv, theta

e $\mathrm{t}$

$\mathrm{t}$

$81 * *$ / wts mm240

02 r102900-8 0 2r307825-8 0 2r510200-8 02 2r708425-8 0

2r901350-8 () 563869-8 316131-8 n2 0 641385-8 359590-8 n2 0

714976-8 400849-8 n2 $0784547-8$ 439853-8 n2 0 857529-8

480771-8 n2 0 642875-8 293289-8 479164-8 n3 $0681415-8$

310872-8 507890-8 n3 0 716550-8 326901-8 534077-8 n3 0

745915-8 340298-8 555965-8 n3 0 775565-8 353825-8 578064-8

n3 $0489468-8$ 386282-8 513536-8 364389-8 n4 $0500102-8$

394674-8 524693-8 372306-8 n4 0 508580-8 401365-8 533587-8

378617-8 n4 0 515474-8 406806-8 540820-8 383750-8 n4 0

517107-8 408094-8 542534-8 384965-8 n4 q120

$82 * *$ / mus mm240

$-641230-7-421582-7 \mathrm{ml}-142963-6-939923-7 \mathrm{ml}-229252-6$

$-150724-6 \mathrm{ml}-315291-6-207291-6 \mathrm{ml}-399349-6-262555-6 \mathrm{ml}$

$-472796-6-411087-6-143488-6 \mathrm{~m} 2-537046-6-466952-6$

$-162988-6 \mathrm{~m} 2-598374-6-520275-6-181600-6 \mathrm{~m} 2-656401-6$

$-570729-6-199211-6 \mathrm{~m} 2-711034-6-618231-6-215791-6 \mathrm{~m} 2$

$-761567-6-713133-6-470428-6-164201-6 \mathrm{~m} 3-807567-6$

$-756207-6-498843-6-174119-6$ m3 -849108-6 -795106-6

$-524503-6-183075-6 \mathrm{~m} 3-885925-6-829582-6-547246-6$

$-191013-6$ m3 -917890-6 -859514-6 -566991-6 -197905-6 m3

Figure E.18 Card Input Data for the DORT Calculation of the ${ }^{60} \mathrm{Co}$

Source Packaged in the 6.5-Inch HRLEL Carrier SNF Shipping Cask. 
$-944812-6-922954-6-765692-6-505099-6-176303-6 \mathrm{~m} 4$ $-966490-6-944130-6-783260-6-516688-6-180348-6 \mathrm{~m} 4$ $-982847-6-960108-6-796516-6-525433-6-183400-6 \mathrm{~m} 4$ $-993815-6-970823-6-805405-6-531297-6-185447-6 \mathrm{~m} 4$ $-999313-6-976194-6-809860-6-534236-6-186473-6$ m4 q 120 $83 * *$ / etas mm240

$3 r-.9979423 r-.9897283 r-.9733673 r-.9489953 r-.916799$

$5 r-.8811725 r-.8435535 r-.8012175 \mathrm{r}-.7544125 \mathrm{r}-.703158$

$7 \mathrm{r}-.6480867 \mathrm{r}-.5897767 \mathrm{r}-.5282227 \mathrm{r}-.4638287 \mathrm{r}-.396835$

9r-.327613 9r-.256704 9r-.184425 9r-. 111045 9r-.037054 g120 $84 \$ \$ 123433242222$ / reg nos by zone

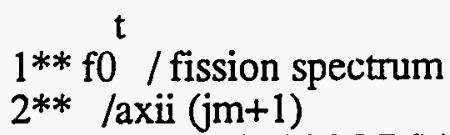

Figure E.18 Continued. 
$13 \mathrm{r} 133 \mathrm{r} 23 \mathrm{q} 46$ /j-ints 263-266

$38 \mathrm{r} 8$ 8r $2 \mathrm{q} 46 / \mathrm{j}$-ints $267-269$

$9 \$ \$ 17132513137257777$ / mat by zone

$24 * *$ fl / importance by zone

$25 \$ \$-31-37-43-49 /$ mats for activity calcs

$26 \$ \$ 11111 / \mathrm{x}$-sec positions for activity calcs

$27 * * 1.01 .01 .01 .0$ / activity multipliers

$28 \$ \$ 61 r 023$ r25 / inners by grp

$29 \$ \$ 22248184117196223250268268268 / \mathrm{j}$-pos key fluxes

$30 \$ \$ 1620393939464646 \quad 1 \quad 1437$ / i-pos key fluxes

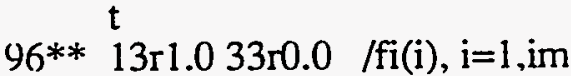

$\mathrm{t}$

$97 * * 48 \mathrm{r} 0.0147 \mathrm{rl} 1.074 \mathrm{r} 0.0 / \mathrm{fj}(\mathrm{j}), \mathrm{j}=1, \mathrm{jm}$

$98 * *$ $61 \mathrm{r} 0.011 \mathrm{r} 0.01 .011 \mathrm{r} 0.0 / \mathrm{fg}(\mathrm{g}), \mathrm{g}=1$, igm

$\mathrm{t}$

Figure E.18 Continued.

$\mathrm{E}-40$ 
Input Changes for 25GWD/MTU LWR MFP Source -....

DORT - 6.5 Inch HRLEL Carrier Calculation, MFP source; $25 \mathrm{GWD} / \mathrm{MTu}$ $63 * *$ ().0 1.39+10 1-4 1-3 1-3 / tmax.xnf.eps,epp,epv

$98 * * 61 \mathrm{r} 0.0 / \mathrm{fg}(\mathrm{g}), \mathrm{g}=1 . \mathrm{igm}$

8.19-03 1.73-(01 1.46-(01 2.53-01 1.18+00 3.49+(00 1.04+01 8.08+(01

$1.93+032.25+(1) 46.36+061.59+082.52+086.96+095.85+079.24+07$

$1.41+084.43+085.22+(185.16+088.70+082.15+091.72+09$

-..-- Input Changes for 50GWD/MTU LWR MFP Source -.-.-

DORT - 6.5 Inch HRLEL Carrier Calculation, MFP source; 50GWD/MTu 63** 0.0 1.47+10 1-4 1-3 1-3 / tmax,xnf,eps,epp,epv

$98 * * 61 \mathrm{r} 0.0 / \mathrm{fg}(\mathrm{g}), \mathrm{g}=1, \mathrm{igm}$

3.10-02 6.53-01 5.53-01 9.54-01 4.44+00 1.32+01 3.91+01 1.73+02

$4.33+032.37+048.85+062.28+083.71+087.23+098.13+079.71+07$

$1.46+084.66+085.95+085.45+088.81+082.26+091.81+09$

Input Changes for Cs-137 Source

DORT - 6.5 Inch HRLEL Carrier Calculation. Cs-137 source

$63 * * 0.03 .467+101-4$ 1-3 1-3 / tmax,xnf,eps,epp,epv

$98 * * 61 \mathrm{r} 0.013 \mathrm{r} 0.01 .09 \mathrm{r} 0.0 / \mathrm{fg}(\mathrm{g}), \mathrm{g}=1, \mathrm{igm}$

Input Changes for ORR MFP Source; 20\% U235

DORT - 6.5 Inch HRLEL Carrier Calculation, ORR MFP Source; 20\% U235 $63^{* *} 0.01 .71+101-41-31-3$ / tmax,xnf,eps,epp,epv

$98 * * 61 \mathrm{r} 0.0 / \mathrm{fg}(\mathrm{g}), \mathrm{g}=1$, igm

4.52-05 9.59-04 8.15-04 1.41-03 6.60-03 1.98-02 5.93-02 2.85+01

$3.75+023.57+043.07+06 \quad 6.03+(071.29+087.86+094.62+071.36+08$

$2.23+086.67+086.48+087.68+081.13+092.92+092.48+09$

---- Input Changes for ORR MFP Source; 40\% U235 -.-.

DORT - 6.5 Inch HRLEL Carrier Calculation, ORR MFP Source; $40 \%$ U235 $63 * * 0.01 .78+101-41-31-3$ / tmax,xnf,eps,epp,epv

$98 * * 61 \mathrm{r} 0.0 / \mathrm{fg}(\mathrm{g}), \mathrm{g}=1, \mathrm{igm}$

4.91-02 1.04+00 8.78-01 1.51+00 7.04+00 2.09+01 6.20+01 2.26+02

$1.41+043.27+048.35+062.20+085.37+088.06+091.35+081.33+08$

$2.07+086.40+087.33+087.37+081.07+092.86+(092.46+09$

Input Changes for ORR MFP Source: 93\% U235 --.-.

DORT - 6.5 Inch HRLEL Carrier Calculation, ORR MFP Source; 93\% U235 $63 * * 0.01 .83+101-4$ 1-3 1-3 / tmax,xnf,eps,epp,epv

$98 * * 61 \mathrm{r} 0.0 / \mathrm{fg}(\mathrm{g}), \mathrm{g}=1, \mathrm{igm}$

1.53-03 3.23-02 2.74-02 4.72-02 2.20-01 6.55-01 1.95+00 2.69+01

$8.68+033.43+(148.45+062.18+085.09+088.03+091.28+081.43+08$

$2.25+086.92+087.78+087.93+081.15+093.02+092.57+(1) 9$

Figure E.19 Card Input Data Changes for the DORT Calculation of the Other Sources Packaged in the 6.5-Inch HRLEL Carrier SNF Shipping Cask. 


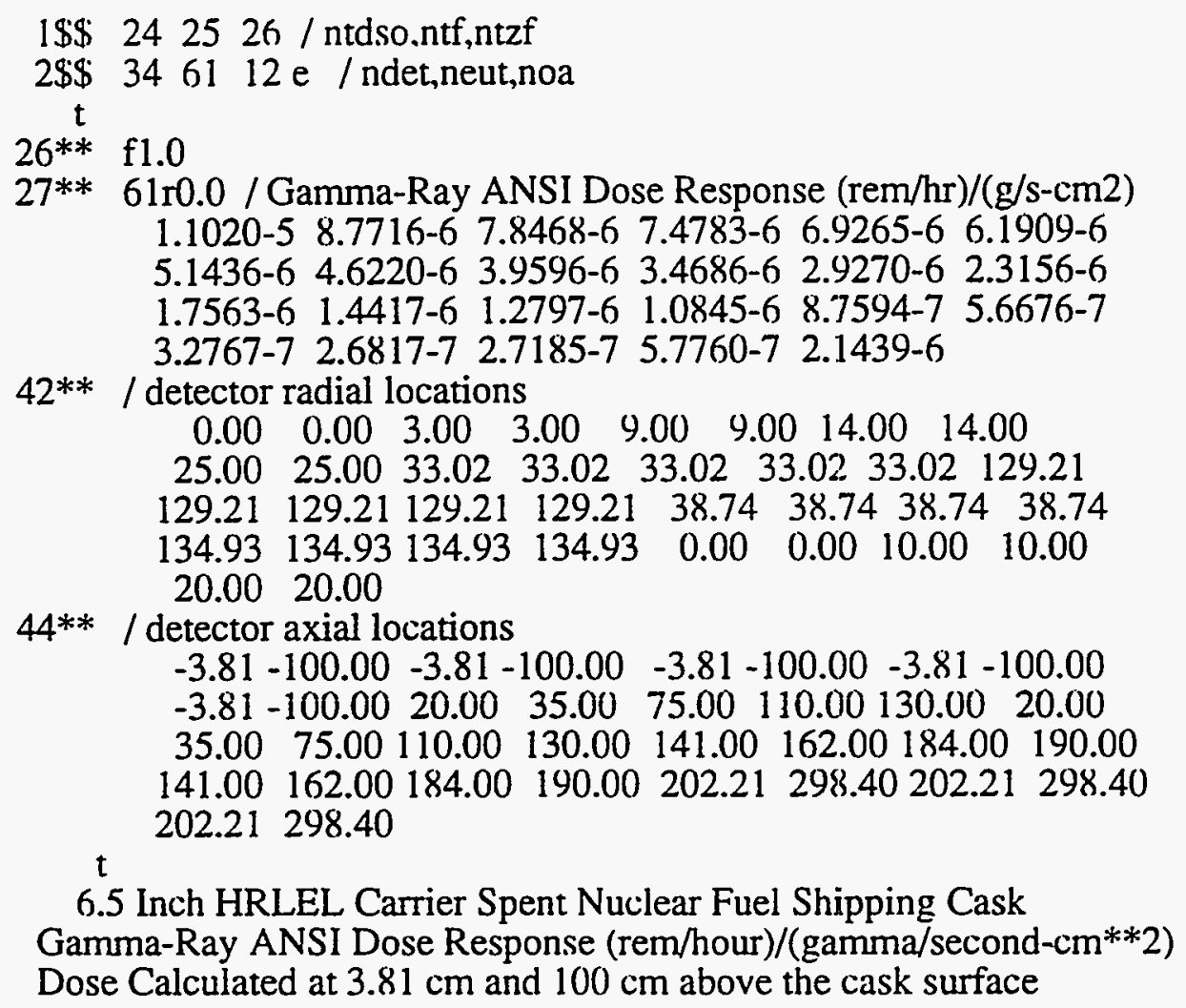

Figure E.20 Card Input Data for the FALSTF Calculations of the Surface and One Meter Distance Dose Rates at the Detector Locations Used in the 6.5-Inch HRLEL Carrier SNF Shipping Cask Shielding Analysis. 
6.5 Inch HRLEL Carrier DORT Geometry Model

OS\$ $2210 \mathrm{e} /$ nflsv, irflx,irfly

$1 \$ \$ 462691200 /$ imx,jm,izm.imap,itip

842540010 / igm,nlvmx,nbuf,igeom,ivol

00000 / ishd,irsp,ihm.nmat.igx

$0 \mathrm{e} /$ ilvck

$2 * * 0.08 .511 .01 .01 .0 /$ size,xpage,ypage,xscl,yscl

$0.00 .00 .00 .00 .0 / x s t p, y s t p, x \min , x \max , y \min$

$0.00 .25 \mathrm{e} / \mathrm{ymax}, \mathrm{eps}$

$t$

$3 \$ \$ 4 \mathrm{f0}$

$4 \$ \$ 1$

$t$

$5 *$ /axii $(\mathrm{jm}+1)$

2i0.0 4i2.54 li6.35 7.94 2i $8.8925 \mathrm{i} 10.8029 .805 \mathrm{i} 30.4434 .57$

$35.52134 i 36.471 i 132.047 \mathrm{i} 133.31139 .98140 .9913 \mathrm{i} 141.88$

$152.685 \mathrm{i} 153.6310 \mathrm{i} 158.395 \mathrm{i} 166.65171 .7413 \mathrm{i} 172.36183 .16$

$184.117 \mathrm{i} 185.062 \mathrm{i} 191.093 \mathrm{i} 193.002 \mathrm{i} 195.86198 .40$

$6^{* *} /$ radii (im+1)

5i0.0 4.12 4.76 4i5.40 8.89 3i9.84 2i12.70 2il14.92 2il 17.15

$10 \mathrm{i} 19.3728 .264 \mathrm{i} 29.21 \mathrm{li} 33.6634 .93$

$8 \$ \$$ / zone numbers by interval

$38 \mathrm{r} 4$ 8r1 2q46/j-ints $1-3$

$18 \mathrm{r} 128 \mathrm{r} 24 \mathrm{q} 46 / \mathrm{j}$-ints $4-8$

18r1 3r2 17r3 1r9 7rl lq46/j-ints 9-10

8r7 10r1 3r2 17r3 1r9 7r1 /j-int 11

6r7 15r2 17r3 1r9 7r1 2q46/j-ints 12-14

6r7 12r5 3r2 17r3 1r9 7r1 25q46 /j-ints 15-40

6r7 2r2 10r5 3r2 17r3 1r9 7rl /j-int 4l

7r1 1r2 10r5 3r2 17r3 1r9 7r1 5q46 /j-ints 42-47

7r1 14r2 17r3 lr9 $7 \mathrm{rl} / \mathrm{j}$-int 48

$13 \mathrm{r} 18 \mathrm{r} 217 \mathrm{r} 31 \mathrm{r} 9 \mathrm{rr} / \mathrm{j}$-int 49

13r1 1r2 24r3 1r9 7r1 134q46/j-ints 50-184

13r1 lr2 24r3 8r9 lq46/j-ints $185-186$

13r1 1r2 30r3 2r9 7q46 /j-ints 187-194

13r1 14r2 17r3 2r9/j-int 195

27r2 17r3 2ry /j-int 196

24r6 3r2 17r3 2r9 13q46/j-ints 197-210

14r11 10r6 3r2 17r3 2r9/j-int 211

13r1 lr10 10r6 3r2 17r3 2r9 5q46 /j-ints 212-217

13r1 1r10 10r6 3r2 17r3 2r9 10q46/j-ints 218-228

$13 r 1$ 1r10 10r6 3r2 17r3 2r9 5q46/j-ints 229-234

$14 \mathrm{r} 12$ 10r6 3r2 17r3 2r9/j-int 235

24r6 3r2 17r3 2r9 13q46 /j-ints 136-249

27r2 17r3 2r9/j-int 250

$13 \mathrm{r} 114 \mathrm{r} 217 \mathrm{r} 32 \mathrm{r} 9 / \mathrm{j}$-int 251

13r1 lr2 30r3 2r9 7q46 /j-ints 252-259

$13 r 133 r 2$ 2q46 /j-ints 260-262

13r1 33r2 3q46/j-ints 263-266

Figure E.21 Card Input Data for the ISOPLOT Isodose Contour Maps for the 6.5-Inch HRLEL Carrier SNF Shipping Cask Shielding Analysis. 
9\$\$17132513137257777/mat by zone

$t$

Radius (cm)\$

Height $(\mathrm{cm}) \$$

6.5 Inch HRLEL Carrier Gamma-Ray Dose Rate\$

$13 \$ \$ 6284100120$ / iga,igb,nlvl,iplt,ipos

$00 \mathrm{e} /$ imat,ichk

$14 * * 1.0-1.0-1.0 \mathrm{e} / \mathrm{fac}, \mathrm{fmin}, \mathrm{fmax}$

$\mathrm{t}$

15** $61 \mathrm{r} 0.0$ / Gamma-Ray ANSI Dose Response (rem/hr)/(g/s.cm2)

1.1020-5 8.7716-6 7.8468-6 $7.4783-6 \quad 6.9265-6 \quad 6.1909-6$

5.1436-6 $4.6220-6 \quad 3.9596-6 \quad 3.4686-6 \quad 2.9270-6 \quad 2.3156-6$

$\begin{array}{lllllll}1.7563-6 & 1.4417-6 & 1.2797-6 & 1.0845-6 & 8.7594-7 & 5.6676-7\end{array}$

$\begin{array}{llllll}3.2767-7 & 2.6817-7 & 2.7185-7 & 5.7760-7 & 2.1439-6\end{array}$

last

6.5 Inch HRLEL Carrier DORT Geometry Model

$1 \$ \$ 462691220$ / imx,jm,izm,imap,itip

842540010 / igm,nlvmx,nbuf,igeom,ivol

00000 / ishd,irsp,ihm,nmat,igx

$0 \mathrm{e} /$ ilvck

2** 0.08 .511 .01 .01 .0 / size,xpage,ypage, xscl,yscl

$0.00 .00 .016 .00 .0 / x s t p, y s t p, x \min , x \max , y \min$ 38.00 .25 e / ymax,eps

$t$

Radius (cm)\$

Height $(\mathrm{cm}) \$$

6.5 Inch HRLEL Carrier End Plug Gamma-Ray Dose Rate\$

$13 \$ \$ 62 \$ 4100120$ /iga,igb,nlvl,iplt,ipos

00 e / imat.ichk

$14 * * 1.0-1.0-1.0 \mathrm{e} / \mathrm{fac}, \mathrm{fmin}, \mathrm{fmax}$

$\mathrm{t}$

15** $61 \mathrm{r} 0.0$ / Gamma-Ray ANSI Dose Response (rem/hr)/(g/s.cm2)

1.1020-5 8.7716-6 7.8468-6 $7.4783-6 \quad 6.9265-6 \quad 6.1909-6$

5.1436-6 $4.6220-6 \quad 3.9596-6 \quad 3.4686-6 \quad 2.9270-6 \quad 2.3156-6$

$\begin{array}{lllllll}1.7563-6 & 1.4417-6 & 1.2797-6 & 1.0845-6 & 8.7594-7 & 5.6676-7\end{array}$

$\begin{array}{llllll}3.2767-7 & 2.6817-7 & 2.7185-7 & 5.7760-7 & 2.1439-6\end{array}$

$\mathrm{t}$

last

6.5 Inch HRLEL Carrier DORT Geometry Model

$1 \$ \$ 462691220 /$ imx,jm,izm,imap,itip

842540010 / igm,nlvmx,nbuf,igeom,ivol

00000 / ishd,irsp,ihm,nmat,igx

$0 \mathrm{e} /$ ilvck

2** $0.08 .511 .01 .01 .0 /$ size,xpage,ypage,xscl,yscl

0.00 .00 .020 .0135 .0 / xstp,ystp,xmin,xmax,ymin 200.00 .25 e / ymax,eps

$\mathrm{t}$

Figure E.21 Continued. 


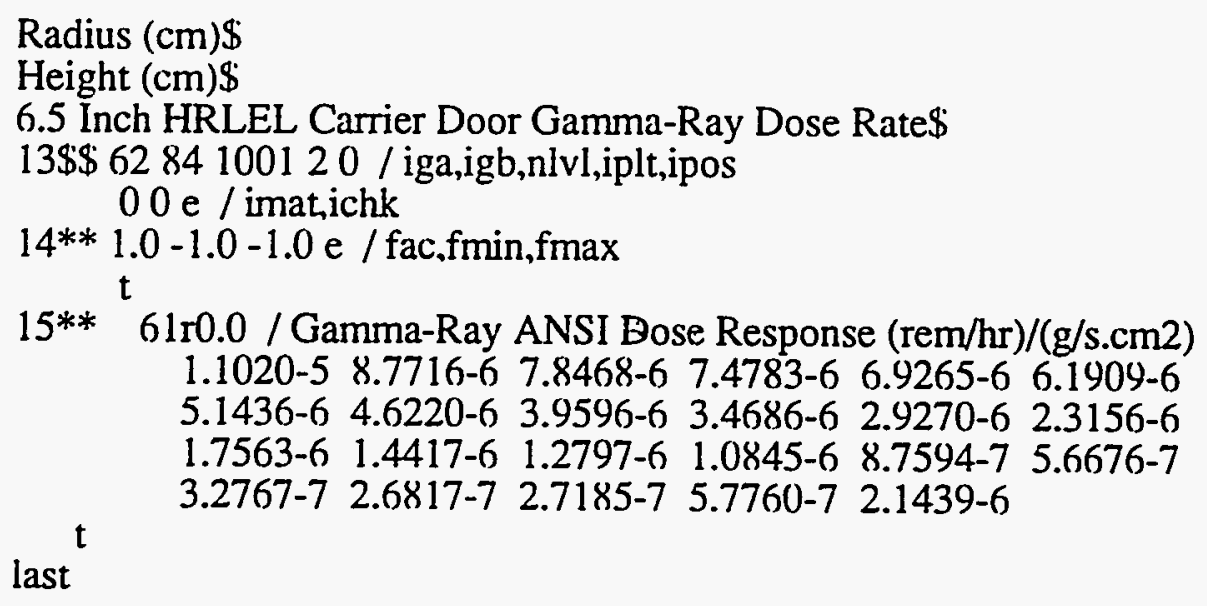

Figure E.21 Continued. 
DORT - HIFR Hot Scrap Shipping Cask Calculation. Co-60 source

' VELM61 61n-23g, P-5, endf/b-v, $\mathrm{mm}=240$ (sym)

$61 \$ \$ 0214023$ / ntflx,ntfog,ntsig,ntbsi.ntdsi

$00000 /$ ntfci,ntibi,ntibo,nenpr,ntdir $24 \mathrm{e} \quad /$ ntdso

$62 \$ \$ 051464243$ / iadj,isctm,izm.im,jm

8434870 / igm,iht,ihs, ihm,mixl

054540240 / mmesh,mtp,mtm,idfac,mm

11000 / ingeom,ibl,ibr,ibb,ibt

$1-1040$ / isrmx,ifxmi,ifxmf,mode,ktype

20003 / iacc,kalf,igtype,inpfxm,inpsrm

$0000-4 /$ njntsr,nintsr,njntfx, nintfx,iact

30110 / ired,ipdb2, ifxprt.icsprt,idirf

00120110 / jdirf,jdirl,nbuf,iepsbz.minblk

01111 / maxblk,isbt,msbt.msdm.ibfscl

450104 / intscl,itmscl,nofis,ifdb2z,iswp

12460000 / keyjn,keyin,nsigtp,norpos,normat

$0115000-10$ / mstmax,negfix, locobj,lcmobj,nkeyfx

$461000 /$ nondin, neut,itally,ispl,isp2

$\mathrm{e}$

$63 * * 0.07 .40+101-41-31-3$ / tmax,xnf,eps,epp,epv

1-3 1.0 0.2 1.5 10.0 /epf,ekobj,evth,evchm,evmax

$1.01 .0-1.00 .310 .0$ /evkmx,evi,devdki,evdelk,sormin

1.0 1-4 1-2 0.0 - 1.5 /conacc,conscl,coneps, wsolmn,wsolii

$1.50 .60 .01-300.0$; wsolcn,orf,fsnacc,flxmin,smooth

1-20.2 0.9 / epo,extrcv, theta

e $\mathrm{t}$

$\mathrm{t}$

$81 * *$ / wts mm240

02 r $102900-802$ 2r307825-8 02 2r510200)-8 02 2r708425-8 0

2 r901350-8 () 563869-8 316131-8 n2 $0641385-8$ 359590-8 n2 0

714976-8 400849-8 n2 0 784547-8 439853-8 n2 0 857529-8

480771-8 n2 0 642875-8 293289-8 479164-8 n3 0 681415-8

310872-8 507890-8 n3 0 716550-8 326901-8 534077-8 n3 0

745915-8 340298-8 555965-8 n3 0 775565-8 353825-8 578064-8

n3 $0489468-8386282-8513536-8364389-8$ n4 $0500102-8$

394674-8 524693-8 372306-8 n4 0 508580-8 401365-8 533587-8

378617-8 n4 0 515474-8 406806-8 540820-8 383750-8 n4 0

517107-8 408094-8 542534-8 384965-8 n4 q120

$82 * *$ / mus mm240)

$-641230-7-421582-7 \mathrm{ml}-142963-6-939923-7 \mathrm{ml}-229252-6$

$-150724-6 \mathrm{ml}-315291-6-207291-6 \mathrm{ml}-399349-6-262555-6 \mathrm{ml}$

$-472796-6-411087-6-143488-6 \mathrm{~m} 2$-537046-6 -466952-6

$-162988-6 \mathrm{~m} 2-598374-6-520275-6-181600-6 \mathrm{~m} 2-656401-6$

$-570729-6-199211-6 \mathrm{~m} 2-711034-6-618231-6-215791-6 \mathrm{~m} 2$

$-761567-6-713133-6-470428-6-164201-6 \mathrm{~m} 3-807567-6$

$-756207-6-498843-6-174119-6 \mathrm{m3}-849108-6-795106-6$

$-524503-6-183075-6 \mathrm{~m} 3-885925-6-829582-6-547246-6$

$-191013-6$ m3 -917890-6 -859514-6 -566991-6 -197905-6 m3

Figure E.22 Card Input Data for the DORT Calculation of the ${ }^{60} \mathrm{Co}$ Source Packaged in the HFIR Hot Scrap Carrier SNF Shipping Cask. 
$-944812-6-922954-6-765692-6-505099-6-176303-6 m 4$ $-966490-6-944130-6-783260-6-516688-6-180348-6 \mathrm{~m} 4$ $-982847-6-960108-6-796516-6-525433-6-183400-6 \mathrm{~m} 4$ $-993815-6-970823-6-805405-6-531297-6-185447-6 \mathrm{~m} 4$ $-999313-6-976194-6-809860-6-534236-6-186473-6$ m4 q120 83** / etas mm240

$3 r-.9979423 r-.9897283 r-.9733673 r-.9489953 r-.916799$

$5 \mathrm{r}-.8811725 \mathrm{r}-.8435535 \mathrm{r}-.8012175 \mathrm{r}-.7544125 \mathrm{r}-.703158$

$7 \mathrm{r}-.6480867 \mathrm{r}-.5897767 \mathrm{r}-.5282227 \mathrm{r}-.4638287 \mathrm{r}-.396835$

$9 \mathrm{r}-.3276139 \mathrm{r}-.2567049 \mathrm{r}-.1844259 \mathrm{r}-.1110459 \mathrm{r}-.037054 \mathrm{~g} 120$

$84 \$ \$ 12322233322222$ / reg nos by zone $1^{* *}$ f0 / fission spectrum

1i0.0 6i0.95 5i5.39 9i9.52 21i16.19 30.16 30.80 149i31.44 2i121.93 li123.83 34i124.78 1i146.69 li147.64 148.91 4** /radii (im+1)

lio.0 $1.3320 \mathrm{i} 2.06 \quad 15.56 \quad 15.88 \quad 16.51 \quad 17.15$ li $17.462 \mathrm{i} 18.73$ $2 \mathrm{i} 20.324 \mathrm{i} 22.2320 \mathrm{i} 25.401 \mathrm{i} 39.0640 .01$ $5 * * \mathrm{f} 1$ / energy group boundaries $8 \$ \$$ / zone numbers by interval 3r2 61r1 lq64/j-ints 1-2 $3 \mathrm{r} 238 \mathrm{r} 10$ 23r1 6q64/j-ints 3-9 3r2 25r3 34r11 2r4 5q64/j-ints 10-15 3r2 25r3 2r11 32r7 2r4 9q64/j-ints 16-25 2r2 26r3 2r11 32r7 2r4 21q64/j-ints 26-47 30r2 32r7 2r4/j-int 48 24r1 Ir12 1r1 4r13 32r7 2r4/j-int 49 24rl 1r12 1r1 1r13 35r7 2r4 149q64/j-ints 50-199 24rl Ir12 Irl 36r13 2r4 2q64/j-ints 200-202 62r5 2r4 lq64/j-ints 203-204 33r8 3r14 26r9 2r4 34q64/j-ints 205-239 $36 \mathrm{r} 1426 \mathrm{r} 92 \mathrm{r} 4$ lq64/j-ints 240-241 $35 \mathrm{rl} 29 \mathrm{r} 6$ lq64 / j-ints 242-243

$9 \$ \$ 171377713131377777 /$ mat by zone $24 * *$ fl / importance by zone $25 \$ \$-31-37-43-49 /$ mats for activity calcs $26 \$ \$ 11111 / \mathrm{x}$-sec positions for activity calcs $27 * * 1.01 .01 .01 .0$ / activity multipliers $28 \$ \$ 61$ r0 23r25 / inners by grp $29 \$ \$ 1331048124202243241241$ / j-pos key fluxes $30 \$ \$ 11730506464645035 \quad$ I/i-pos key fluxes $\begin{array}{rl}96 * * & \begin{array}{l}\mathrm{t} \\ 24 \mathrm{r} 1.0\end{array} \\ \mathrm{t} & 4(\mathrm{r} 0.0 / \mathrm{fi}(\mathrm{i}), \mathrm{i}=1, \mathrm{im} \\ 97 * * & 48 \mathrm{r} 0.0154 \mathrm{rl} .041 \mathrm{r} 0.0 / \mathrm{fj}(\mathrm{j}), \mathrm{j}=1, \mathrm{jm} \\ \mathrm{t} & \\ 98 * * & 61 \mathrm{r} 0.011 \mathrm{r} 0.01 .011 \mathrm{r} 0.0 / \mathrm{fg}(\mathrm{g}), \mathrm{g}=1, \mathrm{igm} \\ \mathrm{t}\end{array}$

Figure E.22 Continued. 
----- Input Changes for 25GWD/MTU LWR MFP Source

DORT - HIFR Hot Scrap Shipping Cask Calculation, MFP source: 25 GWD/MTu $63 * * 0.01 .39+101-41-31-3$ / tmax.xnf.eps,epp,epv

$98 * * 61 \mathrm{r0} .0$ / $\mathrm{fg}(\mathrm{g}), \mathrm{g}=1$,igm

8.19-03 1.73-01 1.46-01 2.53-01 1.18+00 3.49+00 1.04+01 8.08+01

$1.93+032.25+046.36+061.59+082.52+086.96+095.85+079.24+07$

$1.41+084.43+085.22+085.16+088.70+082.15+091.72+09$

----- Input Changes for 50GWD/MTU LWR MFP Source --.--

DORT - HIFR Hot Scrap Shipping Cask Calculation, MFP source; 50GWD/MTu $63 * * 0.01 .47+101-41-31-3$ / tmax,xnf,eps,epp,epv

$98 * * 61 \mathrm{r} 0.0 / \mathrm{fg}(\mathrm{g}), \mathrm{g}=1$,igm

3.10-02 6.53-01 5.53-01 9.54-01 4.44+00 1.32+01 3.91+01 $1.73+02$

$4.33+032.37+048.85+062.28+083.71+087.23+098.13+079.71+07$

$1.46+084.66+085.95+085.45+088.81+082.26+091.81+09$

Input Changes for Cs-137 Source -...-.

DORT - HIFR Hot Scrap Shipping Cask Calculation, Cs-137 source

63**0.03.467+10 1-4 1-3 1-3/tmax,xnf,eps,epp,epv

$98 * * 61 \mathrm{r} 0.013 \mathrm{r} 0.01 .09 \mathrm{r} 0.0 / \mathrm{fg}(\mathrm{g}), \mathrm{g}=1, \mathrm{igm}$

---- Input Changes for ORR MFP Source; 20\% U235

DORT - HIFR Hot Scrap Shipping Cask Calculation, ORR MFP Source; 20\% U235 $63 * * 0.01 .71+101-4$ 1-3 1-3 / tmax,xnf,eps,epp,epv

$98 * * 61 \mathrm{r} 0.0 / \mathrm{fg}(\mathrm{g}), \mathrm{g}=1$, igm

4.52-05 9.59-04 8.15-04 1.41-03 6.60-03 1.98-0.2 5.93-02 2.85+01

$3.75+023.57+043.07+066.03+071.29+087.86+094.62+071.36+08$

$2.23+086.67+086.48+087.68+081.13+092.92+092.48+09$

---- Input Changes for ORR MFP Source; 40\% U235

DORT - HIFR Hot Scrap Shipping Cask Calculation, ORR MFP Source; $40 \%$ U235 $63 * * 0.01 .78+101-41-31-3$ / tmax,xnf,eps,epp,epv

$98 * * 61 \mathrm{r} 0.0 / \mathrm{fg}(\mathrm{g}), \mathrm{g}=1$,igm

$4.91-021.04+008.78-011.51+007.04+002.09+016.20+012.26+02$

$1.41+043.27+048.35+062.20+085.37+088.06+091.35+081.33+08$

$2.07+086.40+087.33+087.37+081.07+092.86+092.46+09$

---- Input Changes for ORR MFP Source; 93\% U235 -..--

DORT - HIFR Hot Scrap Shipping Cask Calculation, ORR MFP Source; 93\% U235 $63 * * 0.01 .83+101-4$ 1-3 1-3 / tmax,xnf,eps,epp,epv

$98 * * 61 \mathrm{r} 0.0 / \mathrm{fg}(\mathrm{g}), \mathrm{g}=1, \mathrm{igm}$

1.53-03 3.23-02 2.74-02 4.72-02 2.20-01 6.55-01 1.95+(00 2.69+(01

$8.68+033.43+048.45+062.18+085.09+088.03+091.28+081.43+08$

$2.25+086.92+087.78+087.93+081.15+093.02+092.57+09$

Figure E.23 Card Input Data Changes for the DORT Calculation of the Other Sources Packaged in the HFIR Hot Scrap Carrier

SNF Shipping Cask. 


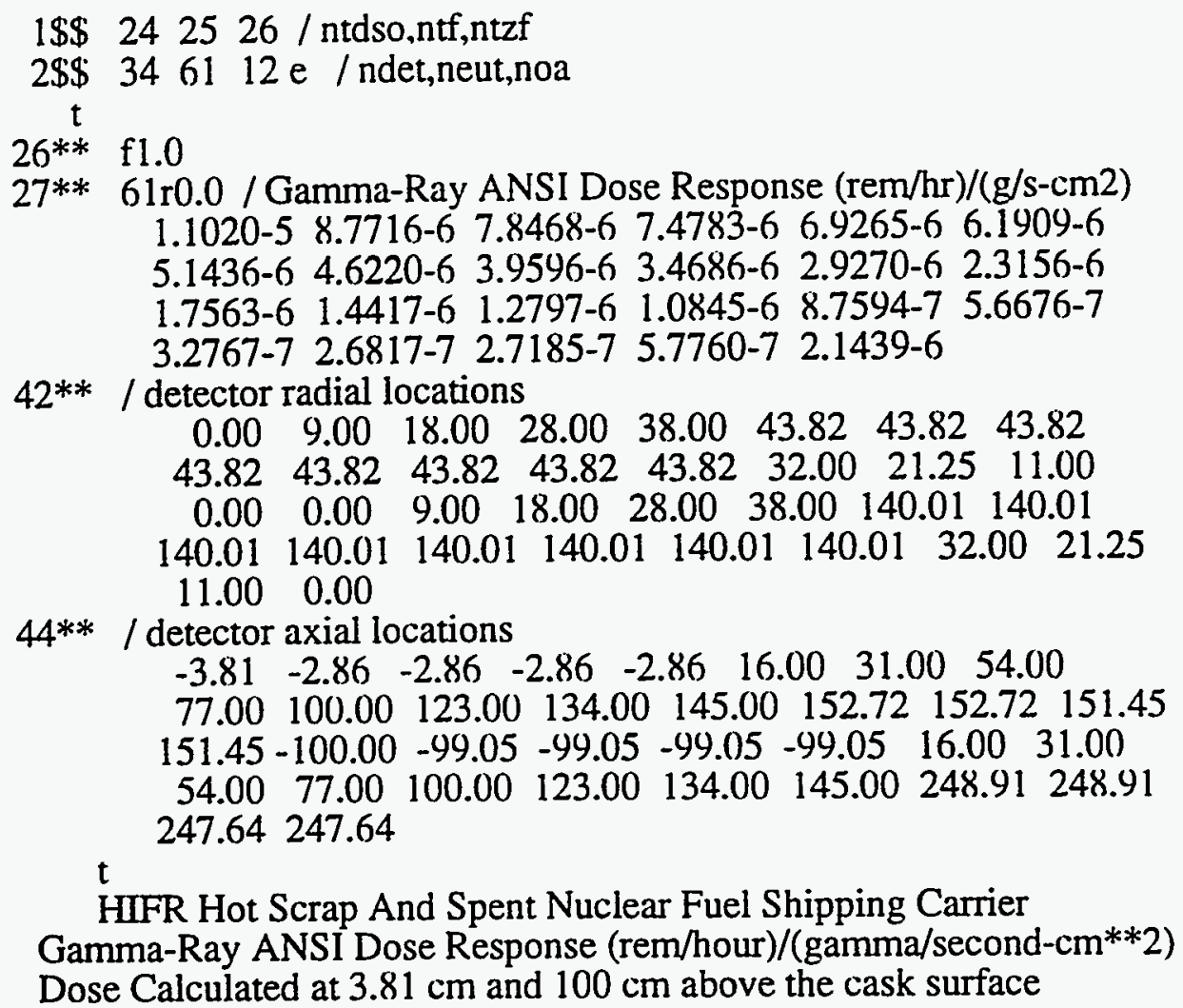

HIFR Hot Scrap And Spent Nuclear Fuel Shipping Carrier Gamma-Ray ANSI Dose Response (rem/hour)/(gamma/second-cm**2) Dose Calculated at $3.81 \mathrm{~cm}$ and $100 \mathrm{~cm}$ above the cask surface

Figure E.24 Card Input Data for the FAISTF Calculations of the Surface and One Meter Distance Dose Rates at the Detector Locations Used in the HFIR Hot Scrap Carrier SNF Shipping Cask Shielding Analysis. 
HIFR Hot Scrap Shipping Carrier DORT Geometry Model

0\$\$ $2210 \mathrm{e} / \mathrm{nflsv}$,irflx,irfly

$1 \$ \$ 642431400 /$ imx,jm,izm,imap,itip

842540010 /igm,nlvmx,nbuf,igeom.ivol

00000 / ishd.irsp,ihm.nmat,igx

0 e / ilvck -

$2 * * 0.08 .511 .01 .01 .0 /$ size,xpage,ypage,xscl.yscl

$0.00 .00 .00 .00 .0 / x s t p, y s t p, x \min , x \max , y \min$

0.00 .25 e / ymax.eps

$$
t
$$

$3 \$ \$ 64 \mathrm{fO}$

$4 \$ \$ \mathrm{fl}$

$5 * * \frac{t}{\text { axii }(j \mathrm{~m}+1)}$

1 i0.0 6i0.95 5i5.39 9i9.52 21i16.19 30.16 30.80 149i31.44

2i121.93 1i123.83 34i124.78 1il46.69 1il47.64 148.91

6** /radii (im+1)

1i0.0 1.33 20i2.06 $15.56 \quad 15.8816 .5117 .151 \mathrm{i} 17.462 \mathrm{i} 18.73$

$2 \mathrm{i} 20.324 \mathrm{i} 22.2320 \mathrm{i} 25.40$ li39.06 40.01

$8 \$ \$$ / zone numbers by interval

3 r2 61r1 lq64/j-ints $1-2$

$3 \mathrm{r} 238 \mathrm{r} 1023 \mathrm{r} 16 \mathrm{~g} 64 / \mathrm{j}$-ints $3-9$

3r2 25r3 34r11 2r4 5q64/j-ints 10-15

3r2 25r3 2r11 32r7 2r4 9q64 /j-ints 16-25

2r2 26r3 2r11 32r7 2r4 21q64/j-ints 26-47

$30 \mathrm{r} 232 \mathrm{r} 72 \mathrm{r} 4 / \mathrm{j}$-int 48

24r1 1r12 lr1 4r13 32r7 2r4/j-int 49

24r1 1r12 Ir1 1r13 35r7 2r4 149q64/j-ints 50-199

24r1 lr12 lr1 36r13 2r4 2q64/j-ints 200-202

62r5 2r4 lq64/j-ints 203-204

$33 \mathrm{r} 83 \mathrm{r} 1426 \mathrm{r} 9 \mathrm{2r} 434 \mathrm{q} 64$ /j-ints 205-239

$36 \mathrm{r} 14$ 26r9 2r4 lq64 /j-ints 240-241

$35 \mathrm{r} 129 \mathrm{r} 6$ la $64 / \mathrm{j}$-ints $242-243$

$9 \$ \$ 171377713131377777 /$ mat by zone

$t$

Radius (cm)\$

Height $(\mathrm{cm}) \$$

HIFR Hot Scrap SNF Shipping Cask\$

$13 \$ \$ 6284100120$ / iga,igb,nlvl,iplt,ipos

$00 \mathrm{e} /$ imat,ichk

$14 * * 1.0-1.0-1.0 \mathrm{e} / \mathrm{fac}, \mathrm{fmin}, \mathrm{fmax}$

$\mathrm{t}$

$15^{* *} 61 \mathrm{r} 0.0$ / Gamma-Ray ANSI Dose Response (rem/hr)/(g/s.cm2)

1.1020-5 8.7716-6 7.8468-6 7.4783-6 6.9265-6 6.1909-6

5.1436-6 4.6220-6 3.9596-6 3.4686-6 $2.9270-6 \quad 2.3156-6$

$\begin{array}{llllll}1.7563-6 & 1.4417-6 & 1.2797-6 & 1.0845-6 & 8.7594-7 & 5.6676-7\end{array}$

$\begin{array}{llllll}3.2767-7 & 2.6817-7 & 2.7185-7 & 5.7760-7 & 2.1439-6\end{array}$

$\mathrm{t}$

last

Figure E.25 Card Input Data for the ISOPLOT Isodose Contour Maps for the HFIR Hot Scrap Carrier SNF Shipping Cask Shielding Analysis. 
HIFR Hot Scrap Shipping Carrier DORT Geometry Model

$1 \$ \$ 642431420 /$ imx,jm.izm,imap,itip

842540010 / igm,nlvmx,nbuf,igeom,ivol

00000 / ishd,irsp,ihm,nmat.igx

$0 \mathrm{e} /$ ilvck

2** 0.08 .511 .01 .01 .0 / size,xpage,ypage, xscl,yscl

$0.00 .00 .025 .0110 .0 / x s t p, y s t p, x \min , x \max , y \min$

155.00 .25 e / ymax,eps

$\mathrm{t}$

Radius (cm)\$

Height $(\mathrm{cm}) \$$

HIFR Hot Scrap SNF Shipping Carrier Bottom Door\$

$13 \$ \$ 6284100120$ / iga,igb,nlvl,iplt,ipos

$00 \mathrm{e} /$ imat,ichk

$14 * * 1.0-1.0-1.0$ e / fac,fmin,fmax

$\mathrm{t}$

$15^{* *} \quad 61 \mathrm{r} 0.0$ / Gamma-Ray ANSI Dose Response (rem/hr)/(g/s.cm2) 1.1020-5 8.7716-6 7.8468-6 7.4783-6 $6.9265-6 \quad 6.1909-6$ 5.1436-6 $4.6220-6 \quad 3.9596-6 \quad 3.4686-6 \quad 2.9270-6 \quad 2.3156-6$ $\begin{array}{lllllll}1.7563-6 & 1.4417-6 & 1.2797-6 & 1.0845-6 & 8.7594-7 & 5.6676-7\end{array}$ $\begin{array}{lllll}3.2767-7 & 2.6817-7 & 2.7185-7 & 5.7760-7 & 2.1439-6\end{array}$

$t$

last

HIFR Hot Scrap Shipping Carrier DORT Geometry Model

$1 \$ \$ 642431420$ / imx,jm,izm,imap,itip

842540010 /igm,nlvmx,nbuf,igeom,ivol

00000 / ishd,irsp,ihm,nmat,igx

$0 \mathrm{e} /$ ilvck

$2 * * 0.08 .511 .01 .01 .0$ / size,xpage,ypage,xscl,yscl

$0.00 .00 .030 .0-5.0 / x s t p, y s t p, x \min , x \max , y \min$ 45.00 .25 e / ymax,eps

t

Radius (cm)\$

Height $(\mathrm{cm}) \$$

HIFR Hot Scrap SNF Shipping Carrier Top Plug\$

$13 \$ \$ 284100120$ / iga,igb,nlvl.iplt,ipos

00 e / imatichk

$14 * * 1.0-1.0-1.0 \mathrm{e} / \mathrm{fac}, \mathrm{fmin}, \mathrm{fmax}$

$\mathrm{t}$

$15 * * \quad 61 \mathrm{r0} 0$ / Gamma-Ray ANSI Dose Response (rem $/ \mathrm{hr}) /(\mathrm{g} / \mathrm{s.cm} 2)$ 1.1020-5 8.7716-6 7.8468-6 7.4783-6 6.9265-6 6.1909-6

$\begin{array}{llllll}5.1436-6 & 4.6220-6 & 3.9596-6 & 3.4686-6 & 2.9270-6 & 2.3156-6\end{array}$

$\begin{array}{lllllll}1.7563-6 & 1.4417-6 & 1.2797-6 & 1.0845-6 & 8.7594-7 & 5.6676-7\end{array}$

$\begin{array}{lllll}3.2767-7 & 2.6817-7 & 2.7185-7 & 5.7760-7 & 2.1439-6\end{array}$

$\mathrm{t}$

last

Figure E.25 Continued. 
DORT - 10 Inch Expt. Removal Shield-ORR Cask Calc.. Co-60 source

'VELM61 61n-23g, P-5, endf/b-v, $\mathrm{mm}=240$ (sym)

$61 \$ \$ 0214023$ /ntflx.ntfog,ntsig,ntbsi.ntdsi $000000 /$ ntfci,ntibi,ntibo,ntnpr.ntdir $24 \mathrm{e}$ / ntdso

$62 \$ \$ 05963641$ / iadj,isctm,izm,im,jm

8434870 /igm,iht.ihs,ihm, mixl

054540240 / mmesh,mtp,mtm,idfac,mm

11000 / ingeom,ibl,ibr.ibb,ibt

1-1040 / isrmx, ifxmi,ifxmf,mode.ktype

20003 / iacc,kalf,igtype,inpfxm,inpsrm

$0000-4$ / njntsr, nintsr,njntfx, nintfx,iact

30110 / ired,ipdb2,ifxprt,icsprt,idirf

00120110 / jdirf,jdirl,nbuf,iepsbz,minblk.

01111 / maxblk,isbt,msbt,msdm,ibfscl

450104 / intscl,itmscl,nofis,ifdb2z,iswp

853000 / keyjn,keyin,nsigtp,norpos,normat

$0115000-10 /$ mstmax,negfix,locobj,lcmobj,nkeyfx

461000 / ncndin,neut,itally,isp1,isp2

$\mathrm{e}$

$63 * * 0.07 .40+101-4$ 1-3 1-3 / tmax,xnf,eps,epp,epv

1-3 1.0 0.2 1.5 10.0 /epf,ekobj,evth,evchm,evmax

$1.01 .0-1.00 .310 .0$ /evkmx,evi,devdki,evdelk,sormin

1.0 1-4 1-2 0.0 -1.5 /conacc,conscl,coneps, wsolmn,wsolii

$1.50 .60 .01-300.0$ /wsolcn,orf,fsnacc,flxmin,smooth

$1-20.20 .9$ / epo,extrcv, theta

e $t$

$81 * *$ / wts mm240

02 2r102900-8 02 2r307825-8 02 2r510200-8 02 2r708425-8 0

2r901350-8 () 563869-8 316131-8 n2 0 641385-8 359590-8 n2 0

714976-8 400849-8 n2 0 784547-8 439853-8 n2 0 857529-8 480771-8 n2 0 642875-8 293289-8 479164-8 n3 0 681415-8 310872-8 507890-8 n3 0 716550-8 326901-8 534077-8 n3 0 745915-8 340298-8 555965-8 n3 0 775565-8 353825-8 578064-8 n3 0 489468-8 386282-8 513536-8 364389-8 n4 0 500102-8 394674-8 524693-8 372306-8 n4 0 508580-8 4.01365-8 533587-8 378617-8 n4 0 515474-8 406806-8 540820-8 383750-8 n4 0 517107-8 408094-8 542534-8 384965-8 n4 q 120

$82 * * /$ mus mm240

$-641230-7-421582-7 \mathrm{ml}-142963-6-939923-7 \mathrm{ml}-229252-6$

$-150724-6 \mathrm{ml}-315291-6-207291-6 \mathrm{ml}-399349-6-262555-6 \mathrm{ml}$

$-472796-6-411087-6-143488-6 \mathrm{~m} 2$ - $537046-6-466952-6$

$-162988-6 \mathrm{~m} 2-598374-6-520275-6-181600-6 \mathrm{~m} 2-656401-6$

$-570729-6-199211-6 \mathrm{~m} 2-711034-6-618231-6-215791-6 \mathrm{~m} 2$

-761567-6 -713133-6 -470428-6 - 164201-6 m3 -807567-6

$-756207-6-498843-6-174119-6 \mathrm{~m} 3-849108-6-795106-6$

$-524503-6-183075-6$ m3 -885925-6 -829582-6 -547246-6

Figure E.26 Card Input Data for the DORT Calculation of the

${ }^{60} \mathrm{Co}$ Source Packaged in the 10-Inch ORR Experiment Removal Shield SNF Shipping Cask. 


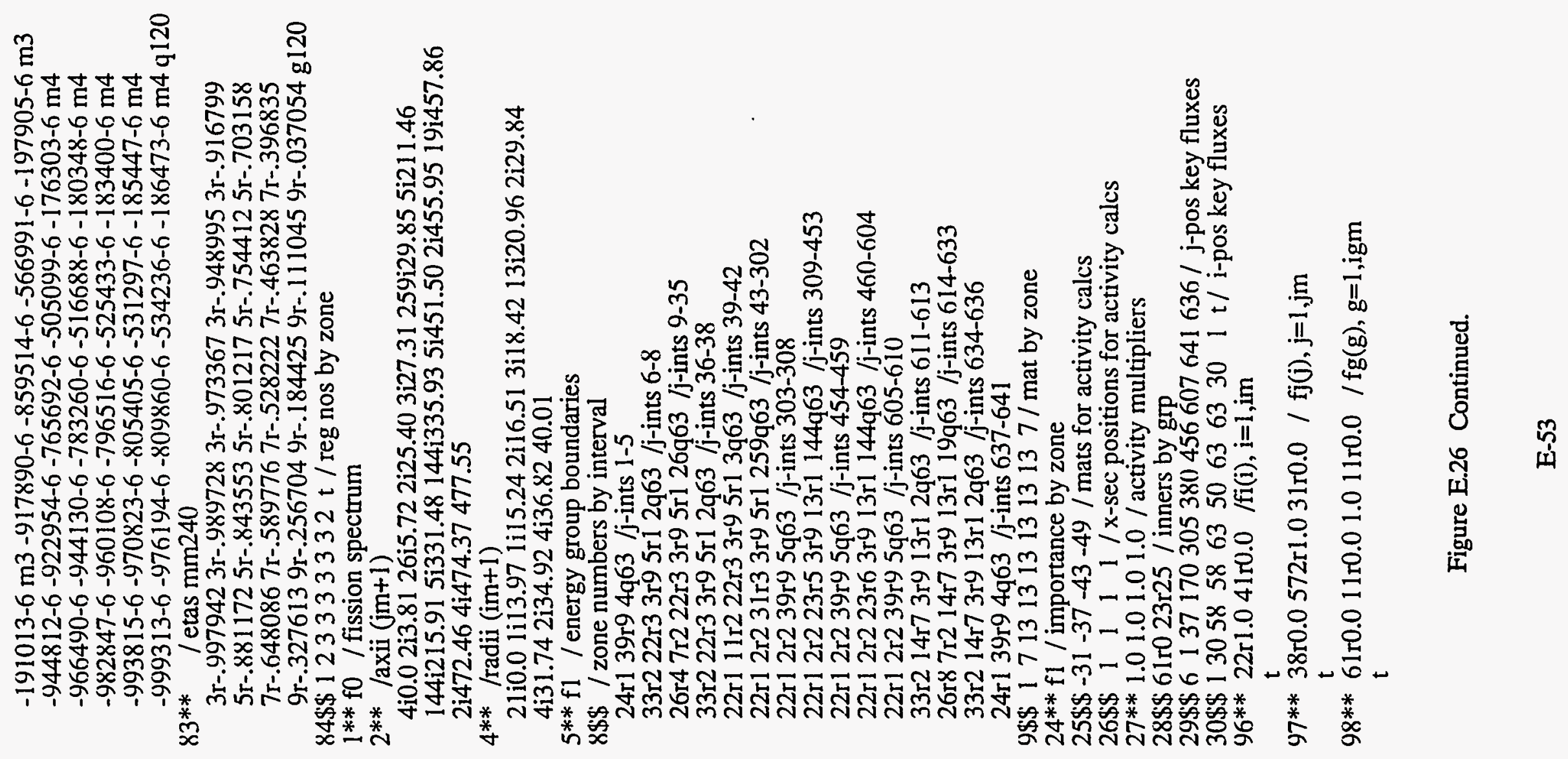


Input Changes for 25GWD/MTU.LWR MFP Source

DORT - 10 Inch Expt. Removal Shield-ORR Cask Calc.,MFP source:25GWD/MTu

$63 * * 0.01 .39+101-41-31-3$ / tmax,xnf.eps,epp,epv

$98 * * 61 \mathrm{r} 0.0 / \mathrm{fg}(\mathrm{g}), \mathrm{g}=1, \mathrm{igm}$

8.19-03 1.73-01 1.46-01 2.53-01 1.18+00 3.49+00 1.04+01 8.08+01

$1.93+032.25+046.36+061.59+082.52+086.96+095.85+079.24+(1) 7$

$1.41+084.43+085.22+085.16+088.70+082.15+091.72+09$

----- Input Changes for 50GWD/MTU LWR MFP Source --.--

DORT - 10 Inch Expt. Removal Shield-ORR Cask Calc.,MFP source;50GWD/MTu 63** 0.0 1.47+10 1-4 1-3 1-3 / tmax,xnf,eps,epp,epv

$98 * * 61 \mathrm{r} 0.0 / \mathrm{fg}(\mathrm{g}), \mathrm{g}=1, \mathrm{igm}$

3.10-02 6.53-01 5.53-01 9.54-01 4.44+00 1.32+01 3.91+01 1.73+02

$4.33+032.37+048.85+062.28+(1) 83.71+087.23+098.13+079.71+07$

$1.46+084.66+085.95+085.45+088.81+082.26+091.81+09$

---- Input Changes for Cs-137 Source -.---

DORT - 10 Inch Expt. Removal Shield-ORR Cask Calc..,Cs-137 source

63** 0.0 3.467+10 1-4 1-3 1-3 / tmax,xnf,eps,epp,epv

$98 * * 61 \mathrm{r} 0.013 \mathrm{r} 0.01 .09 \mathrm{r} 0.0 / \mathrm{fg}(\mathrm{g}), \mathrm{g}=1, \mathrm{igm}$

---- Input Changes for ORR MFP Source; 20\% U235 -----

DORT - 10 Inch Expt. Removal Shield-ORR Cask Calc.,ORR MFP Source;20\% U235 $63 * * 0.01 .71+101-41-31-3$ / tmax,xnf,eps,epp,epv

$98 * * 61 \mathrm{r} 0.0 / \mathrm{fg}(\mathrm{g}), \mathrm{g}=1$, igm

4.52-05 9.59-04 8.15-04 1.41-03 6.60-03 1.98-02 5.93-02 2.85+01

$3.75+023.57+043.07+06 \quad 6.03+071.29+087.86+094.62+071.36+08$

$2.23+086.67+086.48+087.68+081.13+092.92+092.48+09$

---- Input Changes for ORR MFP Source; 40\% U235 --.--

DORT - 10 Inch Expt. Removal Shield-ORR Cask Calc.,ORR MFP Source;40\% U235 $63 * * 0.01 .78+101-41-31-3$ / tmax,xnf,eps,epp,epv

$98 * * 61 \mathrm{r} 0.0 / \mathrm{fg}(\mathrm{g}), \mathrm{g}=1$,igm

4.91-02 1.04+(00 8.78-(01 1.51+(10) 7.04+00 2.09+01 6.20+01 2.26+(1)2

$1.41+043.27+(048.35+(1) 62.20+(0) \times 5.37+088.06+091.35+081.33+(1) 8$

$2.07+086.40+087.33+087.37+081.07+092.86+092.46+09$

---- Input Changes for ORR MFP Source; 93\% U235 --.--

DORT - 10 Inch Expt. Removal Shield-ORR Cask Calc.,ORR MFP Source;93\% U235 $63 * * 0.01 .83+101-41-31-3$ / tmax,xnf,eps,epp,epv

$98 * * 61 \mathrm{r} 0.0 / \mathrm{fg}(\mathrm{g}), \mathrm{g}=1, \mathrm{igm}$

1.53-03 3.23-02 2.74-02 4.72-02 2.20-01 6.55-01 1.95+(00 2.69+(01

$8.68+033.43+048.45+062.18+085.09+088.03+091.28+081.43+08$

$2.25+086.92+(1) 87.78+(1) 87.93+081.15+(1) 93.02+092.57+(19$

Figure E.27 Card Input Data Changes for the DORT Calculation of the Other Sources Packaged in the 10-Inch ORR Experiment Removal Shield SNF Shipping Cask. 
$1 \$ \$ 242526 /$ ntdso,ntf,ntzf

$2 \$ \$ 3461 \quad 12 \mathrm{e} /$ ndet,neut,noa

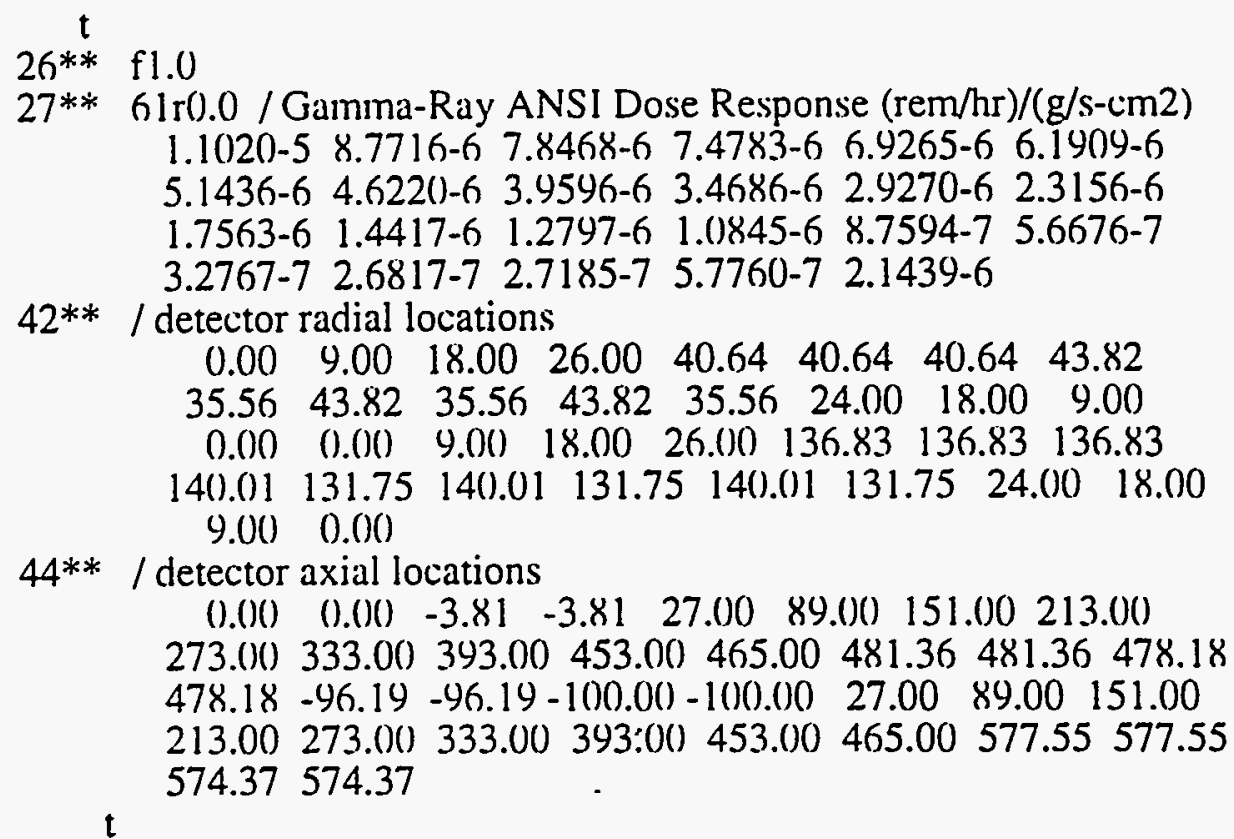

10) Inch Experimental Removal Shield-ORR Spent Fuel Shipping Cask

Gamma-Ray ANSI Dose Response (rem/hour)/(gamma/second-cm**2)

Dose Calculated at $3.81 \mathrm{~cm}$ and $100 \mathrm{~cm}$ above the cask surface

Figure E.28 Card Input Data for the FALSTF Calculations of the Surface and One Meter Distance Dose Rates at the Detector Locations Used in the 10-Inch ORR Experiment Removal Shield SNF Shipping Cask Shielding Analysis. 
10 Inch Expt. Removal Shield-ORR Carrier DORT Geometry Model $0 \$ \$ 2210$ e / nflsv,irflx.irfly

$1 \$ \$ 63641900$ / imx,jm,izm,imap,itip

842550010 / igm,nlvmx,nbuf,igeom,ivol

00000 / ishd.irsp,ihm.nmat,igx

0 e / ilvck

2** $0.08 .511 .01 .01 .0 /$ size,xpage,ypage,xscl.yscl

$0.00 .00 .00 .00 .0 / x s t p, y s t p, x m i n, x \max , y \min$

0.00 .25 e / ymax,eps $\mathrm{t}$

$3 \$ \$ 63 \mathrm{f0}$

$4 \$ \$$ f1

$t$

$5 * *$ /axii $(\mathrm{jm}+1)$

$4 \mathrm{i} 0.02 \mathrm{i} 3.8126 \mathrm{i} 5.722 \mathrm{i} 25.403 \mathrm{i} 27.31259 \mathrm{i} 29.855 \mathrm{i} 211.46$

$144 \mathrm{i} 215.915 \mathrm{i} 331.48144 \mathrm{i} 335.935 \mathrm{i} 451.502 \mathrm{i} 455.9519 \mathrm{i} 457.86$

$2 \mathrm{i} 472.464 \mathrm{i} 474.37477 .55$

6** /radii (im+1)

21i0.0 1i13.97 1i15.24 2i 16.51 3i18.42 13i20.96 2i29.84

$4 \mathrm{i} 31.742 \mathrm{i} 34.924 \mathrm{i} 36.8240 .01$

$8 \$ \$$ / zone numbers by interval

24r1 39r9 4q63 /j-ints $1-5$

33r2 22r3 3r9 5rl 2q63 /j-ints 6-8

26r4 7r2 22r3 3r9 5r1 26q63 /j-ints 9-35

33r2 22r3 3r9 5r1 2q63/j-ints 36-38

22r1 11r2 22r3 3r9 5r1 3q63 /j-ints 39-42

22r1 2r2 31r3 3r9 5r1 259q63 /j-ints 43-302

22r1 2r2 39r9 5q63 /j-ints 303-308

22r1 2r2 23r5 3r9 13r1 144q63 /j-ints 309-453

22r1 2r2 39r9 5q63 /j-ints 454-459

22r1 2r2 23r6 3r9 13r1 144q63 /j-ints 460-604.

22r1 2r2 39r9 5q63 /j-ints 605-610

$33 r 2$ 14r7 3r9 13r1 2q63 /j-ints 611-613

26r8 7r2 14r7 3r9 13r1 19g63 /j-ints 614-633

$33 r 214 r 73 r 9$ 13r1 2q63 /j-ints 634-636

$24 \mathrm{r} 139 \mathrm{r} 94 \mathrm{q} 63$ /j-ints $637-641$

$9 \$ \$ 171313131313137 /$ mat by zone

$\mathbf{t}$

Radius (cm)\$

Height $(\mathrm{cm}) \$$

10 Inch Expt. Removal Shield-ORR SNF Shipping Cask\$

$13 \$ \$ 6284100120$ / iga,igb,nlvl,iplt,ipos

$00 \mathrm{e} / \mathrm{imat}, \mathrm{ichk}$

$14 * * 1.0-1.0-1.0 \mathrm{e} / \mathrm{fac}, \mathrm{fmin} . \mathrm{fmax}$

$\mathrm{t}$

15** $61 \mathrm{r} 0.0$ / Gamma-Ray ANSI Dose Response (rem/hr) $/(\mathrm{g} / \mathrm{s.cm} 2)$

1.1020-5 8.7716-6 7.8468-6 7.4783-6 6.9265-6 6.1909-6

$\begin{array}{llllll}5.1436-6 & 4.6220-6 & 3.9596-6 & 3.4686-6 & 2.9270-6 & 2.3156-6\end{array}$

Figure E.29 Card Input Data for the ISOPLOT Isodose

Contour Maps for the 10-Inch ORR Experiment Removal Shield SNF Shipping Cask Shielding Analysis. 
last

10 Inch Expt. Removal Shield-ORR Carrier DORT Geometry Model

$1 \$ \$ 63641920$ / imx,jm,izm,imap,itip

842550010 / igm,nlvmx,nbuf,igeom.ivol

00000 / ishd,irsp,ihm,nmat,igx

$0 \mathrm{e} /$ ilvck

2** 0.08 .511 .01 .01 .0 / size,xpage,ypage, $x$ scl,yscl

0.00 .00 .00 .00 .0 / xstp,ystp,xmin,xmax,ymin

220.00 .25 e / ymax.eps

$\mathrm{t}$

Radius (cm)\$

Height $(\mathrm{cm}) \$$

10 Inch Expt. Removal Shield-ORR Carrier Heavy Shield\$

$13 \$ \$ 6284100120$ / iga,igb,nlvl,iplt,ipos

$00 \mathrm{e} /$ imat,ichk

$14 * * 1.0-1.0-1.0$ e / fac,fmin,fmax

$\mathrm{t}$

15** 61r0.0 / Gamma-Ray ANSI Dose Response (rem/hr)/(g/s.cm2) 1.1020-5 8.7716-6 7.8468-6 7.4783-6 6.9265-6 6.1909-6

$\begin{array}{cccccc}5.1436-6 & 4.6220-6 & 3.9596-6 & 3.4686-6 & 2.9270-6 & 2.3156-6\end{array}$

$\begin{array}{lllllll}1.7563-6 & 1.4417-6 & 1.2797-6 & 1.0845-6 & 8.7594-7 & 5.6676-7\end{array}$

$\begin{array}{llllll}3.2767-7 & 2.6817-7 & 2.7185-7 & 5.7760-7 & 2.1439-6\end{array}$

last

10 Inch Expt. Removal Shield-ORR Carrier DORT Geometry Model

$1 \$ \$ 63641920 /$ imx,jm,izm,imap,itip

842550010 / igm,nlvmx,nbuf,igeom,ivol

00000 / ishd,irsp,ihm,nmat,igx

$0 \mathrm{e} /$ ilvck

2** 0.08 .511 .01 .01 .0 / size,xpage,ypage,xscl,yscl

$0.00 .00 .00 .0190 .0 / x s t p, y s t p, x \min , x \max , y \min$

350.00 .25 e / ymax,eps

$\mathrm{t}$

Radius (cm)\$

Height (cm)\$

10 Inch Expt. Removal Shield-ORR Carrier Light Shield\$

$13 \$ \$ 6284100120$ / iga,igb,nlvl,iplt,ipos

$00 \mathrm{e} /$ imat,ichk

$14 * * 1.0-1.0-1.0 \mathrm{e} / \mathrm{fac}, \mathrm{fmin}, \mathrm{fmax}$

$\mathrm{t}$

15** $61 \mathrm{r} 0.0 /$ Gamma-Ray ANSI Dose Response (rem/hr)/(g/s.cm2)

1.1020-5 8.7716-6 7.8468-6 7.4783-6 $6.9265-6 \quad 6.1909-6$

5.1436-6 $4.6220-6 \quad 3.9596-6 \quad 3.4686-6 \quad 2.9270-6 \quad 2.3156-6$

$\begin{array}{lllllll}1.7563-6 & 1.4417-6 & 1.2797-6 & 1.0845-6 & 8.7594-7 & 5.6676-7\end{array}$

$\begin{array}{llllll}3.2767-7 & 2.6817-7 & 2.7185-7 & 5.7760-7 & 2.1439-6\end{array}$

last

Figure E.29 Continued. 
10 Inch Expt. Removal Shield-ORR Carrier DORT Geometry Model

$1 \$ \$ 63641920$ / imx,jm,izm.imap,itip

842550010 / igm,nlvmx,nbuf,igeom,ivol

00000 / ishd,irsp,ihm,nmat,igx

0 e Hilvck

2** 0.08 .511 .01 .01 .0 / size.xpage,ypage.xscl.yscl

$0.00 .00 .00 .0320 .0 / x s t p, y s t p, x \min , x \max , y \min$

480.00 .25 e / ymax,eps

$\mathrm{t}$

Radius (cm)\$

Height $(\mathrm{cm}) \$$

10 Inch Expt. Removal Shield-ORR Carrier Light Shield\$

$13 \$ \$ 6284100120$ / iga,igb,nlvl,iplt,ipos

$00 \mathrm{e} /$ imat,ichk

$14^{* *} 1.0-1.0-1.0 \mathrm{e} / \mathrm{fac}, \mathrm{fmin}, \mathrm{fmax}$

$\mathrm{t}$

15** $61 \mathrm{r} 0.0$ / Gamma-Ray ANSI Dose Response (rem/hr) $/(\mathrm{g} / \mathrm{s} . \mathrm{cm} 2)$

1.1020-5 8.7716-6 7.8468-6 7.4783-6 6.9265-6 6.1909-6

5.1436-6 $4.6220-6 \quad 3.9596-6 \quad 3.4686-6 \quad 2.9270-6 \quad 2.3156-6$

$\begin{array}{lllllll}1.7563-6 & 1.4417-6 & 1.2797-6 & 1.0845-6 & 8.7594-7 & 5.6676-7\end{array}$

$\begin{array}{lllll}3.2767-7 & 2.6817-7 & 2.7185-7 & 5.7760-7 & 2.1439-6\end{array}$

$\mathrm{t}$

last

10 Inch Expt. Removal Shield-ORR Carrier DORT Geometry Model

$1 \$ \$ 63641920$ / imx,jm,izm,imap,itip

842550010 / igm,nlvmx,nbuf,igeom,ivol

00000 / ishd,irsp,ihm,nmat,igx

$0 \mathrm{e} / \mathrm{ilvck}$

2** 0.08 .511 .01 .01 .0 / size,xpage,ypage,xscl,yscl

$0.00 .00 .025 .0-5.0 / x s t p, y s t p, x \min , x \max , y \min$

35.00 .25 e / ymax,eps

$\mathrm{t}$

Radius (cm)\$

Height $(\mathrm{cm}) \$$

10 Inch Expt. Removal Shield-ORR Carrier Heavy Door\$

$13 \$ \$ 6284100120$ / iga,igb,nlvl,iplt,ipos

$00 \mathrm{e} /$ imat,ichk

$14 * * 1.0-1.0-1.0 \mathrm{e} / \mathrm{fac}, \mathrm{fmin}, \mathrm{fmax}$

$\mathrm{t}$

$15 * * 61 \mathrm{r} 0.0$ / Gamma-Ray ANSI Dose Response (rem/hr)/(g/s.cm2)

1.1020-5 8.7716-6 7.8468-6 7.4783-6 6.9265-6 6.1909-6

5.1436-6 $4.6220-6 \quad 3.9596-6 \quad 3.4686-6 \quad 2.9270-6 \quad 2.3156-6$

$\begin{array}{llllll}1.7563-6 & 1.4417-6 & 1.2797-6 & 1.0845-6 & 8.7594-7 & 5.6676-7\end{array}$

$\begin{array}{lllll}3.2767-7 & 2.6817-7 & 2.7185-7 & 5.7760-7 & 2.1439-6\end{array}$

$\mathrm{t}$

last

10 Inch Expt. Removal Shield-ORR Carrier DORT Geometry Model

$1 \$ \$ 63641920$ / imx,jm,izm,imap,itip

842550010 / igm,nlvmx,nbuf,igeom,ivol

Figure E.29 Continued.

E-58 


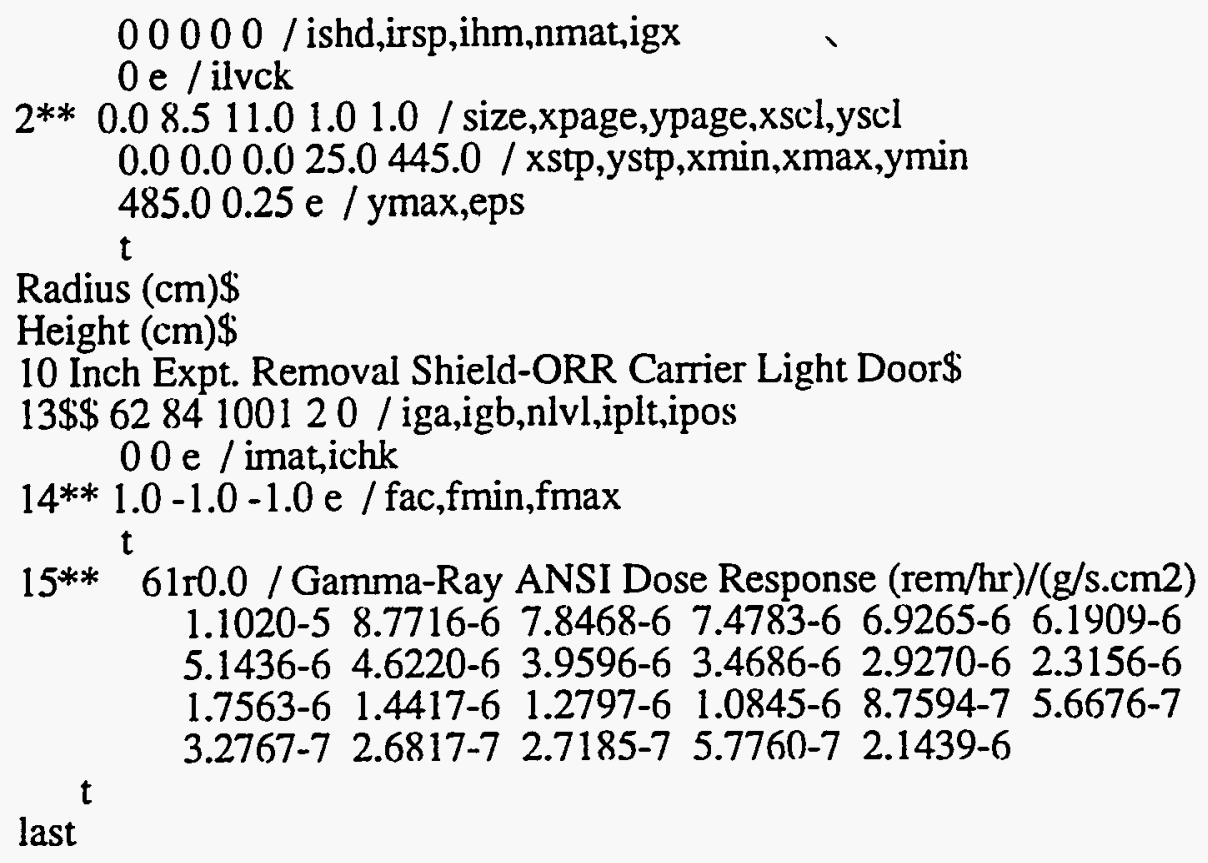

Figure E.29 Continued. 

ORNL/TM-13018

\section{INTERNAL DISTRIBUTION}

1. D. R. Brown

2. R. H. Cooper

3. C. E. DeVores

4. J. D. Drischler

5. T. A. Gabriel

6. D. T. Ingersoll

7-11. J. O. Johnson

12. M. A. Kuliasha

13. G. R. Larson

14. B. C. McClelland

15. R. N. Morris
16. C. E. Oliver

17. N. H. Packan 18-22. J. V. Pace III

23. C. H. Shappert

24. D. W. Turner

25. L. R. Williams

26. Central Research Section

27. Document Reference Section

28. Laboratory Records

29. ORNL Patent Section

\section{EXTERNAL DISTRIBUTION}

30. Office of the Assistant Manager for Energy Research and Development

DOE-ORO

P.O. Box 2008

Oak Ridge, TN 37831-6269

31-32. Office of Scientific and Technical Information P.O. Box 62

Oak Ridge, TN 37830 
